\title{
Mechanisms for Energy Efficient Scheduling-based MAC for Wireless Sensor Networks
}

November, 2009

(C) Copyright 2009, Abdulaziz Y. Barnawi 


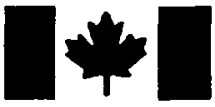

Library and Archives

Canada

Published Heritage Branch

395 Wellington Street

Ottawa ON K1A ON4

Canada
Bibliotheque et

Archives Canada

Direction du

Patrimoine de l'édition

395 , rue Wellington

Ottawa ON K1A ON4

Canada
Your file Votre référence

ISBN: 978-0-494-63861-3

Our file Notre référence

ISBN: 978-0-494-63861-3

\section{NOTICE:}

The author has granted a nonexclusive license allowing Library and Archives Canada to reproduce, publish, archive, preserve, conserve, communicate to the public by telecommunication or on the Internet, loan, distribute and sell theses worldwide, for commercial or noncommercial purposes, in microform, paper, electronic and/or any other formats.

The author retains copyright ownership and moral rights in this thesis. Neither the thesis nor substantial extracts from it may be printed or otherwise reproduced without the author's permission.
AVIS:

L'auteur a accordé une licence non exclusive permettant à la Bibliothèque et Archives Canada de reproduire, publier, archiver, sauvegarder, conserver, transmettre au public par télécommunication ou par l'Internet, prêter, distribuer et vendre des thèses partout dans le monde, à des fins commerciales ou autres, sur support microforme, papier, électronique et/ou autres formats.

L'auteur conserve la propriété du droit d'auteur et des droits moraux qui protège cette thèse. $\mathrm{Ni}$ la thèse ni des extraits substantiels de celle-ci ne doivent être imprimés ou autrement reproduits sans son autorisation.
In compliance with the Canadian Privacy Act some supporting forms may have been removed from this thesis.

While these forms may be included in the document page count, their removal does not represent any loss of content from the thesis.
Conformément à la loi canadienne sur la protection de la vie privée, quelques formulaires secondaires ont été enlevés de cette thèse.

Bien que ces formulaires aient inclus dans la pagination, il n'y aura aucun contenu manquant. 


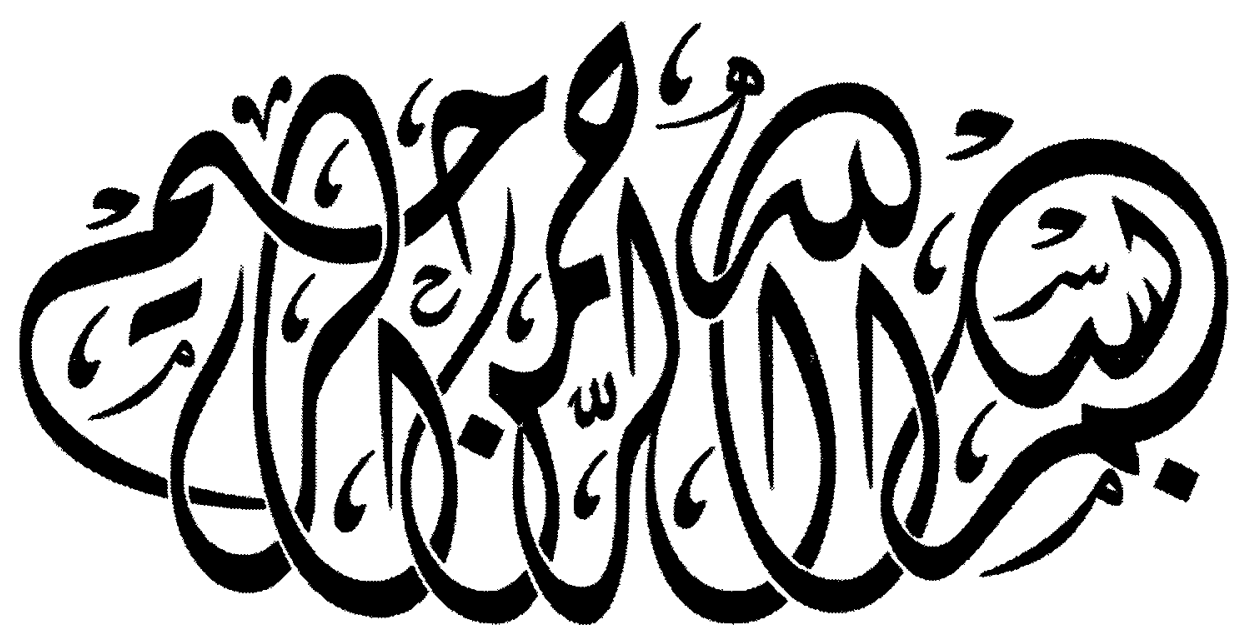




\section{Abstract}

Energy efficient MAC design is a critical objective for wireless sensor networks which are usually highly energy constrained. In addition, the throughput and latency performance is also important for several sensor network applications. Several wireless sensor networks applications are characterized by the many-to-one communications; from sources to sink; instead of the one-to-one mode in ordinary ad-hoc networks. This type of communications might cause congestion at nodes close to the sink. Therefore, congestion avoidance should be used. Reducing data redundancy due to spatial and temporal correlation in sensing data is also an important goal. Further more, major energy waste factors in wireless communications, i.e., idle listening, overhearing, collisions and control overhead, influence the design of MAC protocols. Given these application and protocol design considerations, this dissertation proposes several mechanisms to optimize the energy consumption while maintaining high throughput and application specific latency.

To address the identified energy waste factors, we propose a new centralized multihop scheduling TDMA MAC protocol called On-Demand Convergecast Scheduling based MAC (OCSMACS). OCSMACS supports data collection (source-driven), event-driven and query driven WSN applications with the objective of energy efficiency and delay guarantee. In addition, it employes an adaptive on-demand slot 
assignment, compact and aggregate scheduling requests and integrated routing/MAC. These mechanisms allow nodes to sleep for long periods of time and wake-up at specific time slots to send and/or relay requests for slot assignment to the sink. Based on current topology information, the sink creates a multihop schedule that carries a new slot assignment for the requesting nodes as well as the relaying ones. OCSMACS relies on two proposed spatial-temporal multihop scheduling schemes: Top-Down (TD) and Bottom-Up (BU) scheduling. Both scheduling schemes facilitate the flow of data from nodes to the sink. In addition, they explicitly specify which slot is for send, receive or sleep. Simulations show that OCSMACS outperforms other well-known MAC protocols in terms of latency, throughput and energy efficiency. Furthermore, this performance comes at a scheduling cost that is diminished by the overall gain.

OCSMACS's scheduling setup phase requires performing two main operations: neighbour discovery and topology collection. These operations are carried out by exchanging control messages using CSMA. For applications that require fast network deployment and/or in large or dense sensor networks, the use of CSMA results in contention, large number of collisions and subsequently more time is needed and more and more energy is consumed to collect all topology information. Therefore, we propose a time- and energy-efficient progressive topology construction protocol, called PROGRESSIVE, in which gradual topology information reaches the sink at the same time as TDMA slots are assigned to already discovered nodes. PROGRESSIVE controls the time during which CSMA is used, hence minimizes energy consumption. In addition, nodes can start data transmission as soon they are scheduled by the sink. 
The third part of this dissertation is dedicated to exploiting the presence of correlation in the transmitted data to minimize energy consumption. The proposed spatial-temporal scheduling (TD and $\mathrm{BU}$ ) assumes that each node that receives a data packet needs to forward it as it is. This means that during the data collection period the number of assigned transmit slots is at least equals to the number of receive slots. If data is highly correlated, then few slots are needed to transmit the aggregated data. However, TD and BU assign a considerably higher number of slots than needed. Forcing nodes to wake-up at specific time slots without transmitting or receiving data is a waste of energy and network resources. Therefore, we propose Correlation-Aware (CA) scheduling which allocates enough slots based on the level of data correlation. Two correlation models are considered: Global Correlation model and the proposed Sensing Range Correlation model. Simulation results show that OCSMACS together with the proposed correlation-aware scheduling improves energy efficiency and extends network lifetime beyond what is achievable using TD and BU scheduling. 
To my Father and Mother

To my wife Abeer

To my sons Musab and Yazeed 


\section{Acknowledgments}

In the name of Allah, the Most Gracious and the Most Merciful. Alhamdulillah, all praises to Allah for the strengths and His blessing in completing this dissertation, which without His Willing nothing of this work would have been accomplished.

Upon completing my Ph.D., I would like to thank many people who have helped and inspired me during my doctoral study. People without their help I would not have accomplished this achievement.

Foremost, I would like to express my sincere gratitude to my advisor Prof. Roshdy H.M. Hafez for the continuous support of my Ph.D. study and research, for his patience, motivation, and immense knowledge. His guidance helped me in all the time of research and writing of this dissertation. This dissertation is a small tribute to an exceptional man from a student still anxious to learn from him.

I am very grateful for my years at the Department of Systems and Computer Engineering. I would like to thank the department staff for their excellent assistance and help. Also, thanks to my friends and colleagues at Carleton University; thank you for your support, advice over the past few years.

I also gratefully acknowledge the financial support that I have received while pursuing my Ph.D. In particular, I thank King Fahd University of Petroleum and Minerals (KFUPM), Kingdom of Saudi Arabia for supporting me and my family. 
Without doubt I have been more than fortunate to have this support for the whole duration of my study. I also wish to express my gratitude to the Saudi Arabian Cultural Bureau in Canada for their guidance, support and tremendous efforts.

Where would I be without my family? I owe my loving thanks to my wife Abeer Bargawi, whose dedication, love and persistent confidence in me, has taken the load off my shoulder. My loving thanks to my sons Musab and Yazeed for giving me happiness and joy. My parents deserve special mention for their inseparable support and prayers. My Father (Rahemahu Allah), in the first place is the person who put the fundament education importance, showed me the joy of intellectual pursuit ever since I was a child. I am forever indebted for the guidance and support that he gave me. My Mother, is the one who sincerely raised me with her caring, unconditional love and support. I am also grateful to my brothers, sisters, relatives and friends for being supportive and caring. 


\section{Contents}

List of Tables $\quad$ xiv

List of Figures $\quad$ xvi

List of Acronyms $\quad$ xx

List of Symbols $\quad$ xxiv

1 Introduction $\quad 1$

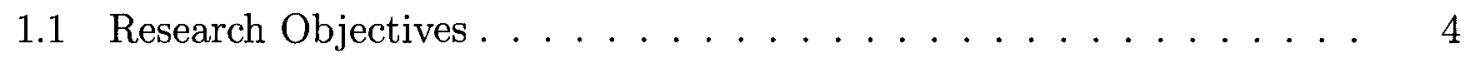

1.2 Contributions ......................... 4

1.3 Dissertation Overview ...................... 7

2 Related Work $\quad 8$

2.1 Energy Consumption in WSNs . . . . . . . . . . . . . . . 8

2.1.1 Sensing Energy .................... 9

2.1.2 Computing Energy . . . . . . . . . . . . . 10

2.1.3 Communication Energy .............. 10

2.2 Medium Access Control for WSN . . . . . . . . . . . . . . 11

2.3 Energy Efficient MAC Protocols . . . . . . . . . . . . . . 17 
2.3.1 Centralized MAC Protocols . . . . . . . . . . . . 17

2.3.2 Distributed MAC Protocols . . . . . . . . . . . . . 18

2.3 .3 Hybrid MAC Protocols . . . . . . . . . . . . . . . . 30

2.4 Multihop Scheduling in Sensor Networks . . . . . . . . . . . . 37

2.5 Summary . . . . . . . . . . . . . . . . . . 39

3 On-demand Convergecast Scheduling-based MAC 41

3.1 Introduction . . . . . . . . . . . . . . . . 41

3.2 Models and Assumptions . . . . . . . . . . . . . . . . . . 44

3.2 .1 Network Model . . . . . . . . . . . . . . . . . . . . 44

3.2 .2 Channel Model . . . . . . . . . . . . . . . . . . 45

3.2 .3 Network Synchronization . . . . . . . . . . . . . . . 46

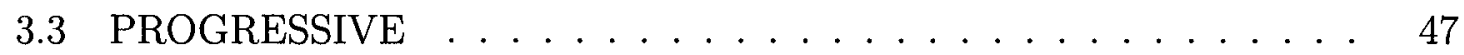

3.3 .1 Neighbor Discovery . . . . . . . . . . . . . . . . 47

3.3.2 Topology Learning and Collection . . . . . . . . . . . . 48

3.3 .3 Adjustment . . . . . . . . . . . . . . . . . . . 49 49

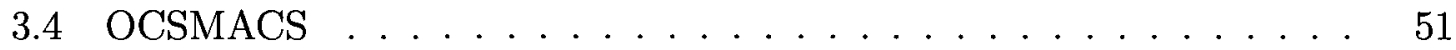

3.4 .1 Frame Structure and Size $\ldots \ldots \ldots \ldots$

3.4 .2 Handling Synchronization $\ldots \ldots \ldots \ldots$

3.4 .3 Requesting Slot Assignment . . . . . . . . . . . . 58

3.4.4 Joining the Network While Operating . . . . . . . . . . . 63

3.5 Multihop scheduling . . . . . . . . . . . . . . . 65

3.5.1 Scheduling Model and Assumptions . . . . . . . . . . . 65

3.5.2 Spatial-temporal Scheduling . . . . . . . . . . . . 67

3.5.3 Leafnode-based Scheduling . . . . . . . . . . . . . . . . 69 
3.6 Summary . . . . . . . . . . . . . . . . . 71

4 OCSMAC Performance Evaluation $\quad 73$

4.1 Introduction . . . . . . . . . . . . . . . . 73

4.2 Scheduling Delay . . . . . . . . . . . . . . . . . . 74

4.2.1 Bottom-Up Scheduling Delay _. . . . . . . . . . 75

4.2.2 Top-Down Scheduling Delay . . . . . . . . . . . . . 76

4.3 Energy Consumption . . . . . . . . . . . . . . . . . . 77

4.3 .1 Radio Energy . . . . . . . . . . . . . . . . . . . 79

4.3.2 Micro-controller Energy _. . . . . . . . . . . . 85

4.3 .3 Optimizing Network Lifetime $\ldots \ldots \ldots \ldots$

4.4 Summary $\ldots \ldots \ldots \ldots \ldots$

$\begin{array}{llr}5 & \text { Simulation Evaluation } & 91\end{array}$

5.1 Introduction . . . . . . . . . . . . . . . . . . . 91

5.2 Assumptions and Default Parameters . . . . . . . . . . . . 92

5.3 PROGRESSIVE Performance . . . . . . . . . . . . . . . 93

5.3.1 NET-LIST Backoff Window Size . . . . . . . . . . . . 95

5.3 .2 Neighbor Discovery Duration $\ldots \ldots \ldots$. . . . . . 97

$5.3 .3 \quad$ Network Size . . . . . . . . . . . . . . . . . . . . . . 100

5.3.4 Relative Network Density . . . . . . . . . . . . . . 102

5.3 .5 Data Rate . . . . . . . . . . . . . . . 106

5.4 OCSMACS Performance . . . . . . . . . . . . . . . . 108

5.4 .1 Network Size . . . . . . . . . . . . . . . . . 110

5.4 .2 Sampling Rate $\ldots \ldots \ldots \ldots$. . . . . . . . 120 
5.5 Discussion . . . . . . . . . . . . . . . . . 127

5.6 Summary . . . . . . . . . . . . . . . . . . 130

6 Correlation-aware OCSMAC 132

6.1 Motivation . . . . . . . . . . . . . . . . . . . 132

6.2 Related Work . . . . . . . . . . . . . . . . . . . 134

6.3 Correlation Models . . . . . . . . . . . . . . . . . . . . 136

6.3 .1 Global Correlation Model . . . . . . . . . . . . . 136

6.3.2 Sensing Range Correlation Model . . . . . . . . . . . . . . 137

6.4 Correlation-aware Scheduling . . . . . . . . . . . . . . 139

6.5 OCSMACS with Correlation-aware Scheduling . . . . . . . . . 140

6.6 Analytical Models . . . . . . . . . . . . . . . . . . . . . . 140

6.6 .1 Scheduling Delay . . . . . . . . . . . . . . . . 141

6.6 .2 Energy Consumption . . . . . . . . . . . . . . . 144

6.7 Simulation Evaluation $\ldots \ldots \ldots \ldots \ldots$

6.7 .1 OCSMACS-GCA $\ldots \ldots \ldots \ldots \ldots \ldots \ldots$

6.7 .2 OCSMACS-SRCA $\ldots \ldots \ldots \ldots \ldots \ldots$

6.7 .3 Aggregation Feasibility . . . . . . . . . . . . . . . . 163

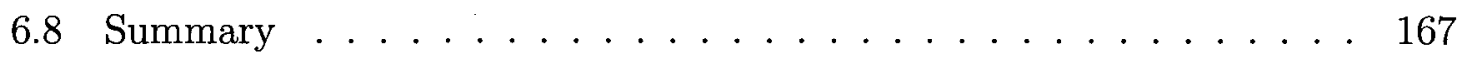

7 Conclusions and Future Work 168

$\begin{array}{lr}\text { A Scheduling Algorithms } & 173\end{array}$

A.1 Interleaved Spatial-Temporal Scheduling . . . . . . . . . . . 174

A.2 Leafnode-based Non-interleaved Scheduling . . . . . . . . . . 175

A.3 Global Correlation-aware Scheduling . . . . . . . . . . 176 
A.4 Sensing Range Correlation-aware Scheduling . . . . . . . . . . . 177

A.5 Update Leafnodes . . . . . . . . . . . . . . . . . 178

A.6 Interference Handling . . . . . . . . . . . . . 178

$\begin{array}{lr}\text { B Confidence-Interval Estimation } & 179\end{array}$

$\begin{array}{ll}\text { C ns-2 Network Simulator } & 181\end{array}$

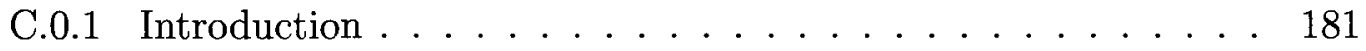

C.0.2 Contributed code . . . . . . . . . . . . . 183

$\begin{array}{lr}\text { Bibliography } & 184\end{array}$ 


\section{List of Tables}

3.1 Maximum clock drifts of MICA2 sensor for different frame sizes. . . . 56

5.1 Default Simulation Parameters. . . . . . . . . . . . . . . . . 93

5.2 MICA2 Sensor Timings and Power Consumption. . . . . . . . . . . 93

5.3 Best parameters for OCSMACS-BU, Z-MAC and S-MAC (maximizing energy efficiency), $r_{\text {sampling }}=0.1 \mathrm{pkt} / \mathrm{sec} \ldots \ldots \ldots \ldots \ldots 111$

5.4 Best parameters for OCSMACS-TD, Z-MAC and S-MAC (minimizing delay to a $\max$ of $1 \mathrm{~s}), r_{\text {sampling }}=0.1 \mathrm{pkt} / \mathrm{sec} \ldots \ldots \ldots 111$

5.5 Best parameters for OCSMACS-BU, Z-MAC and S-MAC, maximizing lifetime, $r_{\text {sampling }}=0.003 \mathrm{pkt} / \mathrm{sec}, 40 \mathrm{~s}$ maximum delay. . . . . . 120

5.6 Best parameters for OCSMACS-BU, Z-MAC and S-MAC for variable $r_{\text {sampling }}, N=100,10$ sources, optimized for energy efficiency. $\ldots . \quad 121$

5.7 Best parameters for OCSMACS-TD, Z-MAC and S-MAC for variable $r_{\text {sampling }}, N=100,10$ sources, optimized for 1 s maximum delay. . . .

6.1 Average number of slots $\tau$ required to schedule 100 nodes for different correlation parameter values. $\operatorname{SEND}_{h(1)}$ is the number of SEND slots assigned to nodes in the first hop. . . . . . . . . . . . . . . 154

6.2 Suggested values of $\rho$ as the distance between nodes changes $\ldots . .159$ 
6.3 Number of slots $\tau$ required to schedule 100 nodes for variable sensing ranges $\mathcal{R}_{\text {sense }} . \mathrm{SEND}_{h(1)}$ is the number of Send slots assigned to nodes in the first hop. . . . . . . . . . . . . . . . 161 


\section{List of Figures}

2.1 Sensor node system architecture. . . . . . . . . . . . . . 9

2.2 The hidden terminal problem. . . . . . . . . . . . . . . . 13

2.3 Periodic listen-and-sleep schedule in SMAC. . . . . . . . . . . . . 21

2.4 A data gathering tree and its DMAC implementation. . . . . . . . 25

2.5 DMAC spatial contention. (a) In DMAC, all child nodes in Level $_{i+1}$ contend to transmit to the same parent in eevel $_{i}$. (b) In S-MAC, child nodes may wake-up at different time intervals to communicate with

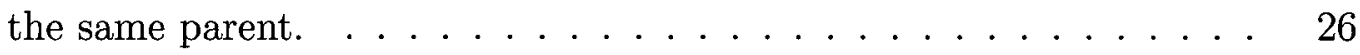

2.6 Low power listening in Z-MAC $\ldots \ldots \ldots \ldots$

3.1 WSN model. . . . . . . . . . . . . . . . . . . . . . . 44

3.2 OCSMAC Frame structure and phases of operation. . . . . . . . 47

3.3 Range of $B W_{N L I S T}$ for different hop counts. . . . . . . . . . . 50

3.4 Topology adjustment. After succeeding in capturing the channel, node B transmits a notification message to node A. Node D starts data transmission only after node B's notification is completely received by

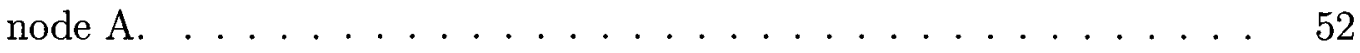

3.5 Sleep frame structure. . . . . . . . . . . . . . . . . 54 


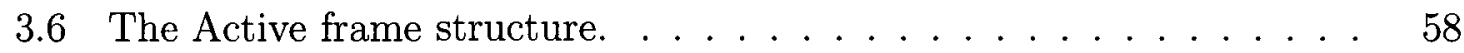

3.7 Schedule request sub-slots. . . . . . . . . . . . . . . . . . 59

3.8 Schedule request. (a) Events A and B are detected in two different parts of a network (b) Request transmission and request aggregation tree for event A (event depth = 3) (c) Request transmission and request aggregation tree for event B (event depth $=2) \ldots \ldots \ldots 61$

3.9 Slot assignment while the network in operation. . . . . . . . . . . 64

3.10 Scheduling model view of the WSN . . . . . . . . . . . . 66

3.11 An example of schedules generated by the two spatial-temporal interleaved scheduling schemes: (a) Top-down (b) Bottom-Up. Nodes which are neither assigned Send nor Receive would be assigned Sleep. 69

3.12 Leafnode-based scheduling for the network example used in Figure 3.11. 70

3.13 Leafnode-based scheduling used to assign listen windows to nodes in Figure 3.11. Node 3 might receive schedule requests during sub-slots 1-4 and then aggregate them for transmission during sub-slot $5 . \ldots 71$

4.1 Multiple rings network representation. . . . . . . . . . 78

5.1 Percentage of nodes which are scheduled from each hop as $R$ is varied, $N=100 . \ldots \ldots \ldots \ldots \ldots \ldots \ldots \ldots \ldots \ldots \ldots \ldots \ldots \ldots \ldots$

5.2 Total number of nodes which are scheduled as $\mathrm{R}$ is varied, $N=100$. . 96

5.3 Simulation results of PROGRESSIVE and DRAND for different $t_{\text {discovery }}$, $N=80 \ldots \ldots \ldots \ldots \ldots \ldots \ldots \ldots \ldots \ldots \ldots \ldots \ldots \ldots \ldots \ldots$

5.4 Number of control message transmissions for different $t_{\text {discovery }}, N=80.99$

5.5 Simulation results of PROGRESSIVE and DRAND for different network sizes, $t_{d i s c o v e r y}=64 \mathrm{~s} . \ldots \ldots \ldots \ldots \ldots \ldots$ 
5.6 Gradual energy consumption of DRAND and PROGRESSIVE, $N=$ $40, t_{\text {discovery }}=16 \mathrm{~s}$. PROGRESSIVE schedules all 40 nodes in about 80s while DRAND in 272s. Once $t_{\text {discovery }}$ ends, PROGRESSIVE's energy consumption is more flat compared to DRAND. . . . . . . . . 103

5.7 Simulation results of PROGRESSIVE and DRAND for different relative network densities, $N=80, t_{\text {discovery }}=64 \mathrm{~s} \ldots \ldots \ldots \ldots$

5.8 Number of control message transmissions for different relative network densities, $N=80, t_{\text {discovery }}=64 \mathrm{~s} \ldots \ldots \ldots \ldots \ldots \ldots \ldots$

5.9 Simulation results of PROGRESSIVE and DRAND for different network sizes, data rate $=2$ Mbps. $\ldots \ldots \ldots \ldots \ldots$

5.10 Simulation results of PROGRESSIVE and DRAND at different relative

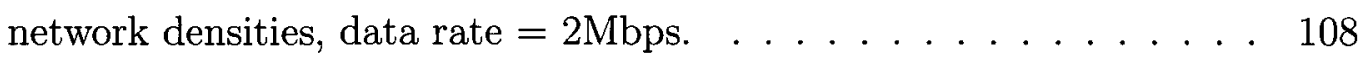

5.11 Number of control message transmissions for different relative network densities, data rate $=2$ Mbps. $\ldots \ldots \ldots \ldots \ldots \ldots$

5.12 Simulation results of OCSMACS-BU, Z-MAC, S-MAC, DMAC and ALWAYS-ON for variable number of nodes. OCSMAC, Z-MAC and S-MAC are all optimized to achieve the best energy efficiency for each network size, $r_{\text {sampling }}=0.1 \mathrm{pkt} / \mathrm{sec} . \ldots \ldots \ldots \ldots \ldots$

5.13 Energy consumed by the radio and the MCU during different modes of operation, $N=100, r_{\text {sampling }}=0.1 \mathrm{pkt} / \mathrm{sec}$, optimized for energy efficiency. . . . . . . . . . . . . . . . . 115

5.14 Energy consumed by nodes at different hops, $N=100, r_{\text {sampling }}=0.1$ pkt/sec, (optimized for energy efficiency). . . . . . . . . . . 
5.15 Scheduling energy and its overhead, $r_{\text {sampling }}=0.1 \mathrm{pkt} / \mathrm{sec}$ (optimized for energy efficiency) . . . . . . . . . . . . . . 116

5.16 Simulation results of OCSMACS-TD, Z-MAC, S-MAC, DMAC and ALWAYS-ON for variable number of nodes. OCSMAC, Z-MAC and S-MAC are all optimized to achieve a 1s maximum end-to-end delay. A delay of more than 1s means that the protocol cannot achieve this requirement at the specific network size, $r_{\text {sampling }}=0.1 \mathrm{pkt} / \mathrm{sec} . \ldots 118$

5.17 Scheduling energy overhead, $r_{\text {sampling }}=0.1 \mathrm{pkt} / \mathrm{sec}$ (optimized for $1 \mathrm{~s}$ maximum delay). . . . . . . . . . . . . . . . . . . 119

5.18 Network lifetime, $r_{\text {sampling }}=0.003 \mathrm{pkt} / \mathrm{sec} \ldots \ldots \ldots \ldots \ldots$

5.19 Simulation results of OCSMACS-BU, Z-MAC, S-MAC, DMAC and ALWAYS-ON for variable sampling rates, $N=100,10$ sources (optimized for energy efficiency). . . . . . . . . . . . . .

5.20 Simulation results of OCSMACS-TD, Z-MAC, S-MAC, DMAC and ALWAYS-ON for variable number of nodes, $N=100,10$ sources. For each sampling rate, OCSMAC, Z-MAC and S-MAC specific parameters are optimized for 1s maximum end-to-end delay. A delay of more than 1s means the protocol cannot achieve this requirement given the specific sampling rate. . . . . . . . . . . . . . .

5.21 Energy efficiency of all protocols, $N=100,10$ sources. Results for both energy-efficiency and 1s end-to-end delay applications. . . . . . . 126

5.22 Energy consumption of nodes at different hops, $N=100,10$ sources, $r_{\text {sampling }}=2 \mathrm{pkt} / \mathrm{sec}$. Results for both energy-efficiency and 1s end-toend delay applications. . . . . . . . . . . . . 
6.1 Nodes A and B are within each other's transmission range, but their data are not necessarily correlated because the monitored target is outside B's coverage area. . . . . . . . . . . . . . . . . 137

6.2 Simulation results of OCSMACS-GCA, OCSMACS-BU+ and OCSMACS$\mathrm{TD}+$ for variable correlation parameter $(\rho) \ldots \ldots \ldots \ldots$

6.3 Energy gain using the GCA Scheduling instead of TD and BU scheduling. 156

6.4 Radio and MCU energy consumption. . . . . . . . . . . . 157

6.5 Network lifetime, $r_{\text {sampling }}=0.003 \mathrm{pkt} / \mathrm{sec} \ldots \ldots \ldots \ldots \ldots$

6.6 Simulation results of OCSMACS-SRCA, OCSMACS-BU+, and OCSMACS$\mathrm{TD}+$ for a variable sensing range. . . . . . . . . . . . 160

6.7 Energy gain using the Correlation-aware scheduling instead of TopDown or Bottom-Up scheduling. . . . . . . . . . . . . . . . . . . 161

6.8 Radio and MCU energy consumption. . . . . . . . . . . . . . . 162

6.9 Network lifetime, $r_{\text {sampling }}=0.003 \mathrm{pkt} / \mathrm{sec} \ldots \ldots \ldots \ldots$

6.10 Energy consumption of OCSMACS-GCA vs. correlation parameter $(\rho)$ for different aggregation times $\left(T_{\text {aggregation }}\right) \ldots \ldots \ldots \ldots$

6.11 Energy consumption of OCSMACS-SRCA vs. sensing range for different aggregation times $\left(T_{\text {aggregation }}\right) \ldots \ldots \ldots \ldots \ldots$

6.12 Communication, processing, and overall energy consumption of OCSMACSGCA for different aggregation processing/compression energy. . . . 166 


\section{List of Acronyms}

\section{Acronym Explanation}

\begin{tabular}{ll}
\hline \hline VLSI & Very Large Integrated Circuits \\
LAN & Local Area Network \\
WSN & Wireless Sensor Network \\
MAC & Medium Access Control \\
CSMA & Carrier Sense Multiple Access \\
TDMA & Time Division Multiple Access \\
CDMA & Code Division Sense Multiple Access \\
FDMA & Frequency Division Multiple Access \\
IEEE & Institute of Electrical and Electronic Engineers \\
OCSMACS & On-demand Convergecast Scheduling-based MAC \\
ALWAYS-ON & CSMAC-based MAC with the radio ALWAYS turned ON
\end{tabular}


RTS

CTS

ACK

T-MAC

STEM

STEM-B

STEM-T

SYNC

MCU

LPL

CCA

NP-hard

AP

BS

ISTS

LNS

BU

TD

OCSMACS-BU OCSMACS with BU scheduling

OCSMACS-TD OCSMACS with TD scheduling

Request To Send

Clear To Send

Acknowledgment

Timeout-MAC

Synchronization

Micro-Controller Unit

Low Power Listening

Clear Channel Assessment

Access Point

Base Station

Bottom-Up multihop scheduling

Top-Down multihop scheduling
Sparse Topology and Energy Management

Sparse Topology and Energy Management-Beacon

Sparse Topology and Energy Management-Tone

Nondeterministic Polynomial-time hard

Interleaved Spatial-Temporal Scheduling

Leafnode-based Non-interleaved Scheduling

$\mathrm{xxi}$ 
OCSMACS-BU+ OCSMACS-BU with opportunistic aggregation OCSMACS-TD+ OCSMACS-TD with opportunistic aggregation GCA Global Correlation-Aware scheduling SRCA Sensing Range Correlation-Aware scheduling OCSMACS-GCA OCSMACS with GCA scheduling OCSMACS-SRCA OCSMACS with SRCA scheduling

SEND OCSMACS transmit slot

ReCeIVe OCSMACS receive slot

Sleep OCSMACS sleep slot

ScheduleReQ OCSMACS schedule request slot Resp/Sync OCSMACS schedule response/syncrhonization slot 


\section{List of Symbols}

\section{Symbol Explanation}

\begin{tabular}{ll}
\hline \hline $\mathcal{P}_{t x}, \mathcal{P}_{r x}$ & Transmit and received power \\
$d_{0}$ & Reference distance \\
$d_{i j}$ & Distance between node $i$ and node $j$ \\
$\alpha$ & Path-loss exponent \\
$G_{t}, G_{r}$ & Transmitter and receiver antenna gain \\
$L$ & System loss factor \\
$\lambda$ & Wavelength \\
$\delta_{s n}$ & Network density \\
$E_{s n}$ & Initial energy of a sensor node \\
$\mathcal{P}_{\text {sink }}, \mathcal{P}_{s n}$ & Sink and sensor node transmit power \\
$\mathcal{R}_{\text {sink }}, \mathcal{R}_{s n}$ & Sink and sensor node transmission range \\
SNR & Signal-to-Noise Ratio \\
&
\end{tabular}


$\eta$

$t_{\text {nextHello }}$ Random time between consecutive HeLlo transmissions

$t_{\text {discovery }} \quad$ Duration of neighbor discovery and partial topology collection

$B W_{N L I S T} \quad$ Size of backoff window for NET-LisT packets

$T_{C C A} \quad$ Time required for CCA

$T_{N L I S T} \quad$ Transmission time of NET-LIST

$T_{\text {DATA }} \quad$ Transmission time of DATA packets

$T_{S C H D} \quad$ Transmission time of SCHDL frame

$T_{\text {Byte }} \quad$ Transmission time of one bytes

$P K T_{\text {length }} \quad$ DATA packet length in bytes

$T_{R E Q} \quad$ Duration of the ScheduleReQ slot

$T_{\text {slot }}^{\text {OCSMACS }}$ OCSMACS-BU, -TD DATA slot duration

$T_{S Y N C} \quad$ Duration of RESP/SYNC slot

$T_{\text {scheduling }}^{\text {sink }} \quad$ Required time to compute a new schedule

$T_{\text {frame }} \quad$ OCSMACS frame duration

$h o p(n) \quad$ Hop count of node $n$

$r_{\text {sampling } \quad \text { Sensor sampling/data generation rate }}$

$\mathcal{S}$

Number of DATA slots in OCSMACS frame

delay $_{\max } \quad$ Maximum end-to-end delay required by the application

$T_{\text {multihop }} \quad$ Delay experienced by a packet during multihop transmission 


\begin{tabular}{|c|c|}
\hline$Q^{\max }$ & Maximum queue size of a node \\
\hline$t_{\text {active-source }}(n)$ & Duration of a timer associated with active sources \\
\hline$T_{\text {join }}$ & Network joining delay \\
\hline $\mathcal{F}_{\text {adjustment }}$ & Frequency of the adjustment phase in a single frame \\
\hline$\tau$ & Time slot \\
\hline $\mathcal{L}$ & List of Leafnodes \\
\hline $\mathcal{D}_{i}(\tau)$ & Number of DATA packets in Level $_{i}$ at the beginning of slot $\tau$ \\
\hline$q_{i}$ & $\begin{array}{l}\text { Proportion of all nodes, which have data in their queues, } \\
\text { in Level } l_{i} \text { and can transmit simultaneously without conflicts } \\
\text { during slot } \tau\end{array}$ \\
\hline$q_{i}^{l}$ & $\begin{array}{l}\text { Proportion of Level }{ }_{i} \text { 's Leafnodes which have data in their } \\
\text { queues, in Level } l_{i} \text { and can transmit simultaneously without } \\
\text { conflicts during slot } \tau\end{array}$ \\
\hline$T_{\text {multihop }}^{\text {BU }}$ & Multihop delay of BU scheduling \\
\hline$T_{\text {multihop }}^{T D}$ & Multihop delay of TD scheduling \\
\hline$T_{\text {multihop }}^{C A}$ & Multihop delay of CA scheduling \\
\hline$\varepsilon$ & Number of request retransmissions \\
\hline$\lambda_{i}$ & Total number of packets generated/sampled by nodes in $r i n g_{i}$ \\
\hline$\lambda_{i}^{t x}$ & $\begin{array}{l}\text { Total number of packets transmitted by nodes in } \text { ring }_{i} \\
\text { and received by nodes in ring ring } g_{i-1}\end{array}$ \\
\hline$\lambda_{i}^{r x}$ & Total number of packets received by nodes in ring $_{i}$ \\
\hline $\mathcal{P}_{\text {listen }}$ & Radio power consumption while idle listening \\
\hline $\mathcal{P}_{\text {switch }}$ & Radio power consumption when activated \\
\hline
\end{tabular}




\begin{tabular}{|c|c|}
\hline $\mathcal{P}_{r f}^{\text {sleep }}$ & Radio power consumption during sleep mode \\
\hline$E_{t x}, E_{r x}, E_{l i s t e n}$ & Radio transmission, reception and idle listening energy \\
\hline$E_{\text {switch }}, E_{\text {sleep }}$ & Radio switching and sleep energy \\
\hline $\mathcal{P}_{\mu C}^{a c t i v e}$ & The MCU power consumption during active operation \\
\hline $\mathcal{P}_{M C U}^{\text {sleep }}$ & The power consumption of the MCU while in sleep mode \\
\hline$E_{M C U}^{a c t i v e}$ & Micro-controller active during communication \\
\hline$E_{M C U}^{\text {sleep }}$ & Micro-controller sleep energy \\
\hline$T_{n e t}$ & Network lifetime \\
\hline$T_{v(i)}$ & Lifetime of node $v$ at the $i^{t h}$ hop from the sink \\
\hline$E^{h(k)}$ & Total energy consumed by a node in the $k^{\text {th }}$ hop from the sink \\
\hline$\lambda_{s n_{i}}$ & Total number of data packets generated by node $s n_{i}$ \\
\hline$\lambda_{\text {sink }}^{s n_{i}}$ & $\begin{array}{l}\text { Total number data packets collected at the sink and originated } \\
\text { from node } s n_{i}\end{array}$ \\
\hline$Q_{s n_{i}(t)}$ & Queue size of node $s n_{i}$ at time $t$ \\
\hline$H_{0}$ & The entropy of any single source \\
\hline$\rho$ & Correlation parameter \\
\hline$\rho_{s_{0} s_{1}}$ & Correlation parameter associated with sources $s_{0}$ and $s_{1}$ \\
\hline $\mathcal{R}_{\text {sense }}$ & Sensing range \\
\hline $\mathcal{D}_{s n_{i}}$ & Total data bytes generated by a node $s n_{i}$ \\
\hline $\mathcal{D}_{\text {neighbors }}$ & Total data bytes received from neighboring child nodes \\
\hline
\end{tabular}


$o$

$T_{\text {aggregation }}$

$T_{\text {slot }}^{O C S M A C S^{+}}$

$p_{i}$

$\lambda_{i}^{G C A^{t x}}, \lambda_{i}^{G C A^{r x}}$

$\lambda_{i}^{S R C A^{t x}}, \lambda_{i}^{S R C A^{r x}}$

$T_{M C U}^{G C A}$

$E_{M C U}^{G C A}$

$E_{M C U}^{\text {aggregation }}$

$\mathcal{G}^{G C A}$

$\mathcal{G}^{S R C A}$
Packet header overhead

Processing time required to carry out aggregation

OCSMACS-CA DATA slot duration

Proportion of Level $_{i}$ 's Leafnodes which have data in their queues and can transmit simultaneously to neighbors within a correlated sensing range without conflict during slot $\tau$

Total number of aggregated packets transmitted and received by ring $_{i}$ in GCA scheduling

Total number of aggregated packets transmitted and received by ring $_{i}$ in SRCA scheduling

Total time during which the MCU is active during communication and data aggregation

Micro-controller energy consumption during communication and data aggregation

Micro-controller energy consumption during data aggregation

Energy gain of GCA scheduling

Energy gain of SRCA scheduling 


\section{Chapter 1}

\section{Introduction}

Advanced developments in VLSI, microprocessor and wireless technology together with increasing interest in applications involving environment monitoring and control (e.g. wildfire and animal habitat monitoring and target tracking in military systems) has lead to the emergence of a new kind of wireless networks, namely sensors in Wireless Sensor Networks (WSNs) where a large number of small sensors are distributed over a field to obtain fine-grained, high-precision sensing data. These networks consist of individual nodes that interact with their environment by sensing physical parameters and applying control functions. In addition, a single node cannot perform a major task so many nodes have to collaborate to fulfill that task. The collected data is transmitted over wireless channels to a central point that makes decisions based on the collected data. Each sensor node contains some computation, wireless communication, sensing and/or control functionalities.

Typically, WSNs operate on batteries. Thus, they are limited in their active lifetime. For this reason, the problem of designing protocols to achieve energy efficiency for extending network lifetime becomes a major concern for network designers. Since 
the radio consumes significantly more power than other components such as sensing and processing, it is critical to conserve energy spent on all radio activities. $[1,2]$

WSNs are expected to support many different real-world applications, e.g. event detection, target tracking and field monitoring. Most of these applications share the following characteristics:

1. There is a clear difference between sources of data, the actual nodes that sense the environment, and sink node(s), where the data should be delivered. Sometimes sink nodes are part of the sensor network itself while sometimes they are terminals outside of the network. Moreover, usually there are more sources than sinks and sinks are not interested in the identity of the sources; the data itself is more important.

Sink nodes can have an unlimited power source. Therefore, protocol design that targets such environments should take advantage of this heterogeneity. In fact an unconstrained power node in a sensor network could play a very important role in the following:

- Time synchronization: in several sensor network applications, providing high precision time synchronization is important due to energy constraints, and the applications' close coupling with the physical world [8]. In addition, a well-synchronized network makes it possible to reduce redundant data by recognizing duplicate detections of the same event by different sensors. The presence of a sink or an Access Point (AP), which periodically sends a message containing its current clock, provides a mechanism for nodes covered by that sink to be synchronized with high accuracy. 
- Energy efficiency: nodes in the network could remain in sleep mode, only being awakened by a signal transmitted by an AP (polling). Furthermore, a TDMA schedule can be created, which allows nodes to sleep and wake-up during specific time slots, therefore conserving energy can be created.

- Task management: an AP can inform nodes about new sensing tasks as well as query sensors for specific measurements.

2. Communications from sources to a sink are characterized by the many-to-one (convergecast) traffic type instead of the one-to-one traffic, in ordinary ad-hoc networks. Convergecast might cause congestion at nodes close to the sink. Therefore, congestion avoidance should be used, for example by distributing the load among these nodes.

3. Nodes collaborate in event detection as well as in reporting their readings and outcomes instead of competing with each other for network resources to achieve these objectives. In addition, the dense deployment of sensor nodes leads to high correlation of the data sensed by the nodes in the neighborhood. To eliminate data redundancy, collaborative efforts should be made among sensor nodes to filter data locally according to the requirement. Data aggregation and compression could be performed to increase energy efficiency.

Traditional design of wireless ad-hoc network protocols is based on the layered design approach. The advantage of this approach lies in its simplicity, ease of standardization and flexibility to deploy new protocols. However, the design of each layer in the stack is isolated and the interface between layers is static and independent of network and application constraints. In WSN energy, memory and computational 
power are greatly constrained resources. These constraints, together with the application specific characteristics of sensor networks, impact multiple network layers. Thus, a cross-layer design approach is more desirable in that it exploits the interactions between layers and promotes adaptability at all layers, based on information exchange between layers, is more desirable.

\section{$1.1 \quad$ Research Objectives}

The main objective of this dissertation is to propose and evaluate scheduling-based protocols for low power wireless sensor networks. This dissertation addresses the following issues:

1. Achieving high energy efficiency by reducing the effect of factors such as collisions, idle listening and overhearing.

2. Reducing data redundancy.

3. Balancing the energy consumption and prolong the average battery life of individual sensors by improving the distribution of tasks among neighboring nodes.

4. Adapting efficiently to parameters such as node density, topology and sampling rates.

\subsection{Contributions}

Our main contribution lies in demonstrating the performance gains in the domain of WSN's MAC. These gains are achieved by exploiting various low-power schedulingbased design mechanisms for convergecast traffic applications. 
Our contributions can be summarized as follows:

1. An energy efficient MAC layer protocol called On-Demand Convergecast Schedulingbased MAC (OCSMACS) is proposed. OCSMACS allows nodes to sleep most of the time and wake-up at specific time slots to send and/or relay requests for slot assignment to the sink. Based on current topology information, the sink creates a multihop schedule that carries a new slot assignment for the requesting nodes as well as the relaying ones.

2. Two underlying multihop scheduling schemes are proposed: Top-Down and Bottom-Up scheduling.

3. The topology construction and the scheduling setup phases are essential parts of creating a multihop scheduling-based MAC. In the context of WSN, PROGRESSIVE, an energy and time efficient topology construction and schedule setup protocol, is proposed.

4. A Sensing Range Correlation model is proposed. Based on this model, a correlation-aware scheduling scheme is set out, along with a Global Correlationaware scheduling.

5. Using the correlation-aware scheduling schemes, a correlation-aware OCSMACS protocol is proposed. This OCSMACS has the ability to improve energy efficiency and extends network lifetime based on a knowledge of existing correlation in the transmitted data.

PROGRESSIVE and OCSMACS are implemented in $n s-2$ [9]. The performance of PROGRESSIVE is evaluated and compared against that of DRAND [10]. Likewise, 
OCSMACS is evaluate and compared with S-MAC [11], DMAC [12], Z-MAC [13] and a CSMA fully awake protocol (ALWAYS-ON). Results show that OCSMACS outperforms other protocols in various performance aspects while providing throughput and latency performance comparable to ALWAYS-ON protocol. The correlation-aware OCSMACS provides a significant performance improvement in terms of energy efficiency and network lifetime compared to the noncorrelation-aware version.

The following is a list of published and submitted papers:

1. Abdulaziz Barnawi and R.H.M Hafez, "PROGRESSIVE: A Topology Discovery and Scheduling Protocol for Wireless Sensor Networks," to appear in The 12-th ACM International Conference on Modeling, Analysis and Simulation of Wireless and Mobile Systems, MSWiM'09, Oct. 2009, Spain.

2. Abdulaziz Barnawi and R.H.M Hafez, "A Time \& Energy Efficient Topology Discovery and Scheduling Protocol for Wireless Sensor Networks," to appear in Proc. of The 2009 IEEE/IFIP International Symposium on Embedded and Pervasive Systems, August 29-31, Vancouver, Canada.

3. Abdulaziz Barnawi and R.H.M Hafez, "TDMA Scheduling for Data Gathering in Wireless Sensor Networks," Proc. International Conference on Wireless Networks (ICWN). World Congress in Computer Science, Computer Engineering, and Applied Computing, WorldComp'08, vol. 1, pp. 94 - 100, July 2008.

4. Abdulaziz Barnawi and R.H.M Hafez, "Performance of TDMA Scheduling Algorithms in the Presence of Data Correlation in Sensor Networks," Proc. IEEE Canadian Conference on Electrical and Computer Engineering, IEEE CCECE, pp. 675-680, May 2008. 
5. Abdulaziz Barnawi and R.H.M Hafez, "An Adaptive MAC Protocol for Infrastructure Wireless Sensor Networks," Proc. International Conference on Sensor Technologies and Applications, SENSORCOMM, pp. 301 - 306, Oct. 2007.

\subsection{Dissertation Overview}

The rest of this dissertation is organized as follows: Chapter 2 provides a background and an overview of past work related to this dissertation. In Section 2.1, the problem of energy consumption in wireless sensor networks is discussed. Section 2.2 highlights main issues related to MAC design in the context of WSN. Section 2.3 classifies energy-aware MAC protocols into three categories; centralized, distributed and hybrid. Examples of each category are presented. In Chapter 3, PROGRESSIVE and OCSMACS protocols are discussed. In addition, the underlying scheduling schemes (Top-Down and Bottom-Up) are presented. An analytical assessment of OCSMACS is presented in Chapter 4. Meanwhile, Chapter 5 presents $n s$ - 2 simulation-based evaluation of PROGRESSIVE and OCSMACS. Results are compared to those of DRAND, Z-MAC, S-MAC, DMAC and ALWAYS-ON. Chapter 6 discusses energy-efficient data correlation-aware scheduling schemes. Two data correlation models are presented, Global Correlation and Sensing Range Correlation models. Based on these models, two multihop scheduling schemes are proposed; Global Correlation-aware scheduling and Sensing Range Correlation-aware scheduling. Analytical models and simulation results for the proposed correlation-aware protocols are also presented in Chapter 6 . Chapter 7 summarizes the conclusions and presents directions for future research. 


\section{Chapter 2}

\section{Related Work}

This chapter, discusses the energy efficiency challenge in sensor networks and its effect on MAC protocol design. In addition, a detailed summary of related previous work is presented. In doing so, the main focus is on protocols and algorithms which exploit various mechanisms to extend wireless sensor networks' lifetime.

Section 2.1 discusses typical sensor node architecture and each component's contribution to energy consumption. Section 2.2 deals with MAC protocol design and its relation to energy efficiency in sensor networks. Section 2.3 presents a classification of MAC protocols: centralized, distributed and hybrid. Finally, Section 2.4 addresses multihop scheduling in sensor networks.

\subsection{Energy Consumption in WSNs}

A typical sensor node is composed of: a power supply unit, sensing unit, computing/processing unit and communication unit [19, 20, 21, 22], Figure 2.1. Except for the power unit, all other components consume energy when performing their tasks. 


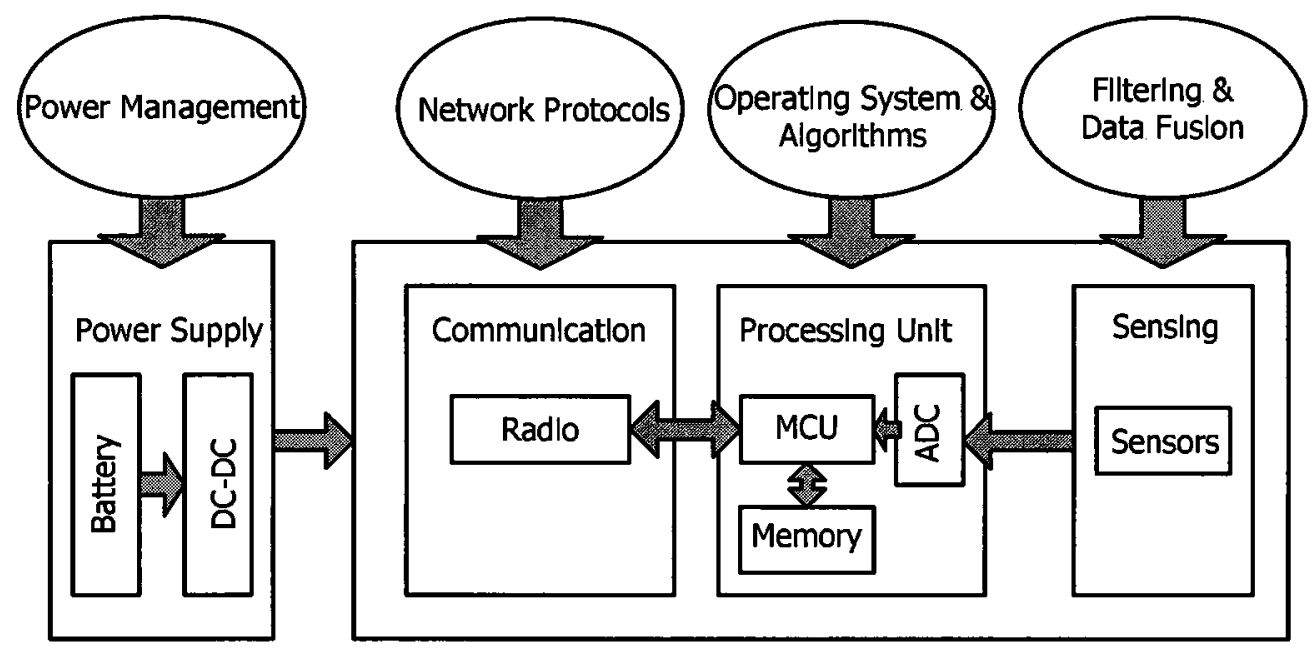

Figure 2.1: Sensor node system architecture.

\subsubsection{Sensing Energy}

The sensing unit in a sensor node includes embedded sensor, possibly an actuator and the analog-to-digital converter. It is responsible for capturing the physical characteristics of the sensed environment and converting its measurements to digital signals, which can be processed by a computing/processing unit [22]. The energy consumed for sensing includes: (1) physical signal sampling and conversion to an electrical signal; (2) signal conditioning; and (3) analog to digital conversion. To reduce sensing energy, interval sensing can be used, i.e. turning off the nodes in inactive duty cycles. However, there is an overhead when transitioning from an inactive state to active one. Regardless, sensing energy represents only a small percentage of the total power consumption in a WSN. For example, MICA2 Mote sensors [23, 24] consume $15 \mathrm{~mW}$ of power when sensing is taking place and $15 \mu \mathrm{W}$ when the sensor is off. 


\subsubsection{Computing Energy}

The computing/processing unit is an MCU or a microprocessor with memory. It collects data from sensors, processes the data, decides when and where to send it and process received data from other sensor nodes [2]. The computing/processing executes various programs, ranging from time-critical signal processing and communication protocols to application programs. Low power circuit design plays an important role in reducing computational energy. In addition to that, the controller should be turned off as long as possible (a MICA2 micro-controller consumes $24 \mathrm{~mW}$ during full operation and $45 \mu \mathrm{W}$ when it is in sleep mode). A preprogrammed timer can be used to activate the node periodically or when an actual event is detected. However, it is important to take into account that transitions between these operating modes involve a power and latency overhead.

\subsubsection{Communication Energy}

The communication unit in a sensing node mainly consists of a short-range RF circuit which performs data transmission and reception. The communication energy, determined by the total communication and transmission distance, is the major contributor to the total energy expenditure. Communication in WSNs is affected by several factors, including type of modulation scheme, data rate and transmit power. In general, radios can operate in four distinct modes of operation: transmit, receive, idle and sleep. Operating in idle mode for a long duration of time results in high power consumption almost equal to that in receive mode [21]. Therefore, it is important for power saving purposes to switch transceivers between different states, for example - active and sleeping. For instance, a MICA2 radio consumes $81 \mathrm{~mW}$ when 
transmitting, $30 \mathrm{~mW}$ during - receive/idle modes and $3 \mu W$ sleeping. Minimizing the amount of data communicated among sensors and transforming a long transmission distance into a number of short ones are key steps in optimizing the communication energy [22].

\subsection{Medium Access Control for WSN}

In wireless networks, nodes share a single medium for communication. Network performance is largely affected by how efficiently and fairly nodes can share this common medium. A fundamental task of any MAC protocol is to coordinate the access of a number of nodes to a shared medium so that certain application-dependent performance requirements are satisfied. $[22,2]$

Traditionally, the most important performance attributes of MAC protocols are throughput, stability, fairness, low access delay and low transmission delay as well as a low overhead. The later could result from per-packet overhead (MAC headers and trailers), collisions or from exchange of extra control packets. However, these metrics cannot be fully met and some of them are conflicting. Designers need to make tradeoffs among different attributes based on the application requirements. [2, 51]. In the following section of this chapter, these attributes are discussed; the extent to which they affect the design of MAC protocols for WSN is also explained.

Collision avoidance - the operation and performance of MAC protocols is heavily influenced by the properties of the underlying physical layer. High error rate caused by physical phenomena such as fading, path loss, attenuation, noise and interference, is an inherit problem in wireless transmission. In addition, wireless 
waveforms propagating through free space are subject to a distance-dependent loss of power called path loss. The received power $\left(\mathcal{P}_{r x}\right)$ at a distance $d \leq d_{0}$ between transmitter and receiver can be expressed (the general case) as:

$$
\mathcal{P}_{r x}(d)=\mathcal{P}_{r x}\left(d_{0}\right) \cdot\left(\frac{d_{0}}{d}\right)^{\alpha}
$$

where $d_{0}$ is the reference distance depending on the antenna technology, $\alpha$ is the path-loss exponent typically varying between 2 (free-space path loss) and 5 to 6 (shadowed areas and obstructed in-building scenarios) [52]. $P_{r x}\left(d_{0}\right)$ can be calculated using the Friis free-space equation:

$$
\mathcal{P}_{r x}\left(d_{0}\right)=\frac{\mathcal{P}_{t x} G_{t} G_{r} \lambda^{2}}{(4 \pi)^{2} d_{0}^{2} L}
$$

where $\mathcal{P}_{t}$ is transmission power, $G_{t}$ and $G_{r}$ are antenna gains of the transmitter and receiver, $\lambda$ is wavelength and $L \geq 1$ is system loss factor (losses through transmit/receive circuitry). Path loss combined with the requirement any transceiver needs a minimum signal strength to successfully demodulate signals results in a maximum range which a sensor node can be reached by a given transmit power.

In CSMA-based protocols if two nodes are out of reach, they cannot hear each other's transmission which gives rise to the well-known hidden-terminal problem. As Figure 2.2 shows when a node $\mathrm{A}$ wants to transmit to a node $\mathrm{B}$ it senses the medium and if it is idle it transmits. However, a node $\mathrm{C}$ is not able to hear node $\mathrm{A}$ and transmit to $\mathrm{B}$ at the same time. This causes packets collisions at node B. These collisions could lead to inability of the receiver to correctly 
decode a packet, hence causing the upper layers of the sender to perform a retransmission.

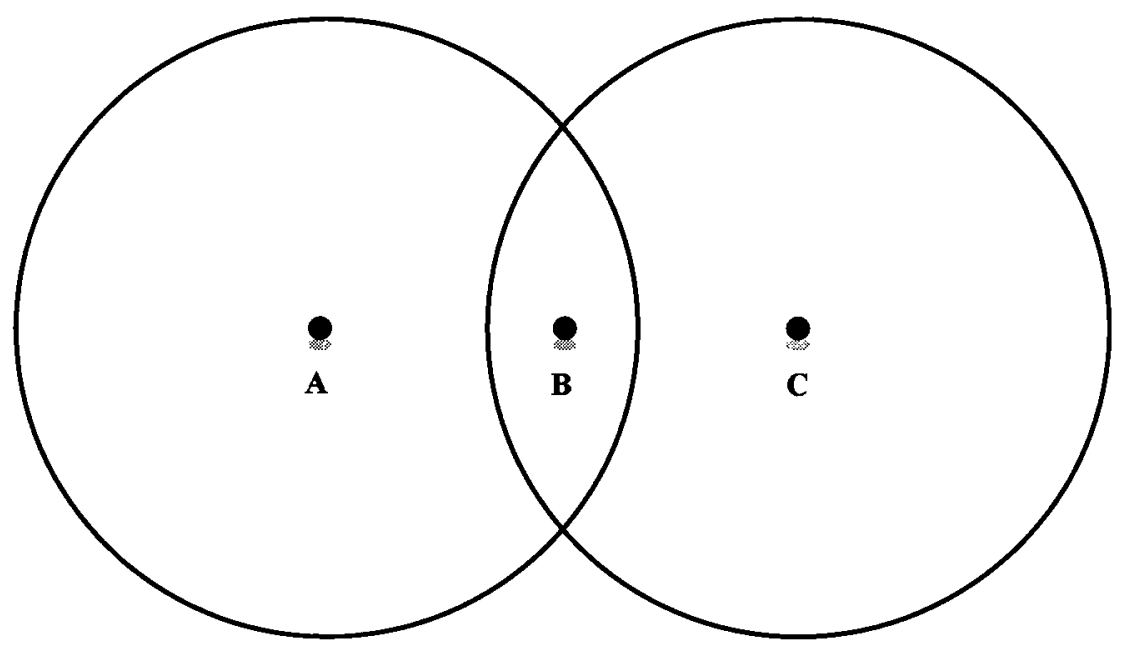

Figure 2.2: The hidden terminal problem.

Therefore, collision avoidance is a fundamental function of all wireless ad-hoc and sensor networks MAC protocols. It determines when and how a node can access the shared medium and send its data. Completely avoiding collisions is not always possible. Contention-based MAC protocols accept some level of collisions, but all MAC protocols avoid frequent ones. Corrupted packets have to be discarded and possible follow-on-retransmissions increase energy consumption and latency. In the context of WSNs, energy efficiency is extremely critical. According to previously discussed characteristics, nodes of a WSN have extremely low energy resources and remain unattended after deployment. In fact, some design goals of sensor networks include building nodes cheap enough to be discarded rather than recharged or efficient enough to operate using ambient power sources. Even though some exhausted nodes could be compensated for by using redundant neighboring nodes, in certain situations, a part of the network 
might become completely inactive or inaccessible and isolated from the other parts due to low connectivity and insufficient coverage. Therefore, MAC protocols for WSNs should be carefully designed to achieve an optimal performance toward the intended application.

Scalability and adaptivity are closely related attributes of a MAC protocol exhibiting changes in network size, node density and topology. Some nodes might die over time and new ones might join the network at a later time of operation. A good MAC protocol should easily accommodate such changes. Scalability and adaptivity to changes in size, density and topology are important attributes because sensor networks are deployed in an ad-hoc manner and often operate in uncertain environments.

Latency refers to the delay in time from when a sender has a packet to be sent until the packet is successfully received by the destination. In sensor networks, the importance of latency depends on the application. In applications such as surveillance or monitoring, nodes are largely inactive until something is detected. These applications could often tolerate certain additional messaging latency because a network speed is typically orders of magnitude faster than the speed of a physical object. The speed of a sensed object places a bound on how rapidly a network must react. During periods of sensing event absence, normally there is very little data flowing in a network. Sub-second latency for an initial message after an idle period might be less important than potential energy savings and longer operational lifetime. However, after event detection, low latency operation becomes more important. 
Throughput (often measured in bits or bytes per second) refers to the amount of data successfully transferred from a sender to a receiver in a certain amount of time. Many factors affect the throughput, including efficiency of collision avoidance, channel utilization, latency and control overhead. Likewise latency, the importance of throughput depends on the specific application. Sensor applications demanding long lifetime often accept longer latency and lower throughput.

Fairness reflects the ability of different users, nodes or applications to equally share the channel. It is an important attribute in traditional voice or data network since each user desires an equal opportunity to send or receive data for their own applications. However, in sensor networks, all nodes cooperate for a single common task. At any particular time, one node may have more data to send than some of the other nodes. Thus, rather than treating each node equally, success is measured by the performance of the application as a whole and pernode or per-user fairness becomes less important.

Energy Efficiency in wireless sensor networks requires a stringent control of energy consumption throughout network operation time. The design of MAC protocols for such networks needs to be as efficient as possible in terms of energy consumption. The next section presents a detailed overview of energy consumption and sources of energy waste in MAC protocols.

\section{Sources of Energy Waste}

As previously discussed a transceiver could be in one of the four main states: transmitting, receiving, idling or sleeping. Measurements indicate that transmitting is costly; receiving costs often have the same order of magnitude as transmit costs; 
idling could be significantly cheaper but also about as expensive as receiving; and sleeping costs almost nothing. In the context of MAC protocols for WSNs, there are four main sources of energy waste $[11,53,54,55]$ :

- Idle listening occurs when a radio is listening to a channel to receive possible data which has not been already sent. The cost is especially high in many sensor network applications where there is not any data to send during periods without any network activity. However, in many MAC protocols such as IEEE 802.11 ad-hoc mode or CDMA, nodes have to listen to the channel to receive possible traffic. Measurements have shown that idle listening consumes $50 \%-100 \%$ of the energy required for receiving [11]. According to [56], the ratio of $E_{\text {idle }}$ : $E_{\text {receiving }}: E_{\text {transmitting }}$ is $1: 2: 2.5$. Since most sensor networks are designed to operate for long periods of time, nodes remain idle for long stretches of time. This is why, idle listening is a major cause of energy waste in such cases.

- Overhearing occurs when a node receives packets destined for other nodes. Overhearing unnecessary traffic could be a main cause of energy waste when traffic load is heavy and network density high. However, according to [2], sometimes overhearing is desirable, for example, when collecting neighborhood information or estimating current traffic load for management purposes.

- Collision - When two packets collide they become corrupted and must be discarded. Possible follow-on retransmissions consume energy as well. Because of limited capacity of the wireless channel, data losses take place when traffic is heavy and the network encounters congestion which also might require retransmissions. While collision is a major problem in contention protocols, generally, this is not the case in scheduled protocols. 
- Control packet overhead includes sending, receiving and listening for control packets, for instance RTS and CTS packets or request packets in demand assignment protocols.

In order to design an energy-efficient MAC protocol, collisions must be avoided

as much as possible. Moreover, energy dissipation due to idle channel listening, overhearing and control overhead should also be reduced to a minimum. In order to reduce energy consumption, most of the MAC protocols developed for WSN tackle one or more of these problems. However, it is difficult to achieve all energy-conserving objectives at the same time.

\subsection{Energy Efficient MAC Protocols}

MAC protocols can be classified into distinct groups according to different criteria. Based on whether a central controller is involved in coordination, they can be classified as centralized (Section 2.3.1), distributed (Section 2.3.2) and hybrid (Section 2.3.3).

\subsubsection{Centralized MAC Protocols}

Centralized MAC protocols, such as FDMA, CDMA and TDMA, achieve a collisionfree operation by using a master controller to coordinate channel access among the different nodes; hence, no special mechanisms are needed to avoid hidden-terminal situations [2]. In addition, the use of these multiple access schemes enables nodes to turn off their radio during unassigned slots, thus, saving energy expenditure otherwise incurred by listening and overhearing.

However, these schemes also have disadvantages. For example, TDMA setup 
and maintenance of schedules involve high signalling traffic, especially when network topology changes due to mobility or failure of nodes. Additionally, a schedule of a node (and possibly these of its neighboring nodes) might require a significant amount of memory which is a scarce resource. Maintaining time synchronization also involves some extra signalling traffic and by extension extra energy. Frequent resynchronization of nodes is indeed required due to clock drifts resulting from low cost oscillators used in sensors. An increase in the number of synchronization messages raises energy consumption of the central controller compared to other nodes. A further disadvantage is that schedules are not easily adapted to different load situations on small timescales. With respect to FDMA, it is not realistic to assign a unique frequency for each individual node in large WSNs due to the limited bandwidth of the system. Likewise, in the case of CDMA, an overhead results from the need to encode nodes' data bits with uniquely assigned codes.

\subsubsection{Distributed MAC Protocols}

In contrast to centralized protocols, distributed MAC protocols usually provide random multiple access to a wireless medium without relying on a master node to coordinate their operations. Most of the distributed MAC protocols rely on CSMA channel access scheme. In this scheme, after a node gets a new packet for transmission from

its upper layers, it waits for a random delay and then performs carrier sensing. If the medium is busy, the node goes into backoff mode. In the backoff mode, the node waits a random period of time which depends on the number of channel access trials it has attempted until this point. After the end of the backoff mode the node listens again; if the medium is busy and the node has exhausted its maximum number of 
trials, the packet is dropped. If the medium is idle, the node sends its data packet and waits for an acknowledgment.

CSMA/CA is used to prevent or minimize the number of collisions during data transmission. In this respect it is similar in its operation to CSMA. However, when the node finds the medium idle, instead of sending data it transmits an RTS packet and waits for a corresponding CTS packet. In case CTS is not received or one for a different pair of nodes is received, the node either enters backoff mode or drops the packet depending on the limits of packet transmission retries. If the CTS packet is received the node sends its data packet and waits for an ACK.

CSMA/CA protocols (e.g. the IEEE 802.11) are not suitable for limited power nodes like those used in WSNs because they require:

- any node $x$ to be constantly in listen mode since another node $y$ may attempt to transmit a frame to node $x$ at any time.

- all nodes to overhear RTS and CTS packets in order to properly adjust their Network Allocation Vector (NAV) timers.

As already discussed, both idle listening and overhearing are two major energy waste factors. Even though IEEE 802.11 has some power-saving functionalities [58], the protocol is targeted towards high bitrate applications and the available transceivers consume orders of magnitude of energy than it is acceptable for low bitrate sensor network applications. Furthermore, IEEE 802.11 has goals which do not match those of wireless sensor networks; a main objective is allowing a number of independent and competing users to share a common channel in a fair manner.

Several distributed MAC protocols have been proposed to address the idle listening problem. The following part of this section describes two categories of these protocols: 
Duty Cycle Protocols and Wake-up On-demand Protocols as well as their advantages and disadvantages.

\section{Duty cycle MAC protocols}

During network operation, duty cycle protocols allow nodes to alternate between two periods: listen period and sleep period. During the listen period, nodes listen to the channel for possible traffic from neighbors and go back into sleep mode when that period expires. A potential transmitting node must acquire knowledge about listen periods of targeted receiving nodes and send its packet at the right time. An entire cycle consisting of a sleep and listen period is called a wakeup period. The ratio of the listen period length to the wakeup period length is called duty cycle. It is important to note that a short duty cycle means that a node sleeps most of the time to avoid idle listening and conserve energy. However, as the duty cycle gets shorter, the listen period also gets shorter, which might increase contention among nodes wanting to transmit to the same target node, especially in heavy load situations. In addition a long sleep period increases latency. Examples of duty cycle protocols are: S-MAC [11], T-MAC [53], and DMAC [12].

S-MAC [11] is the first protocol to adopt the idea of duty cycle operation. Its design considers the following assumptions [22]:

- Short-range multihop communications take place among a large number of nodes.

- Most communications are one-to-one between nodes as peers, rather than manyto-one (nodes to a single base station). 
- In-network data processing is used to reduce traffic.

- Collaborative signal processing and data aggregation reduce traffic and improve sensing quality.

- Low bitrate, high latency applications utilize the network.

All nodes in a network alternate between a fixed-length listen period and fixedlength sleep period according to a pre-selected schedule. Each sleep-listen cycle is called a frame, see Figure 2.3. Sending or receiving a packet is performed only during a listen period. Therefore, neighboring nodes need to coordinate their schedules so that their listen periods start at the same time.

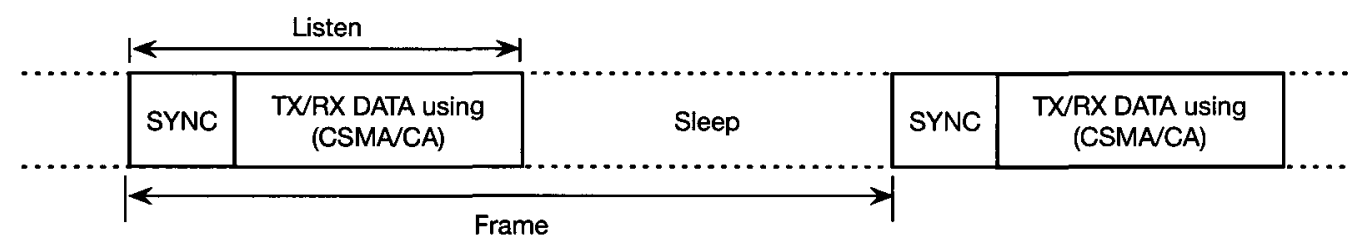

Figure 2.3: Periodic listen-and-sleep schedule in SMAC.

In addition, the listen period is subdivided into two main phases. In the first phase, called SYNC phase, nodes exchange schedules by periodically broadcasting a SYNC packet to their immediate neighbors (by using CSMA). If a node receives a schedule from a neighbor before choosing or announcing its own schedule, it adopts the received schedule. Thus, it can wake at appropriate listen times and send its own schedule. However, if a node receives a different schedule after it has announced its own schedule, it must do one of the following:

- If the node does not detect other neighbors, it can discard its current schedule and adopt the new one. 
- If the node has one or more neighbors, it can adopt both schedules by waking up at the listen intervals of both.

A set of nodes synchronized together form a virtual cluster. Since each node is allowed to have different schedules it could be a member of more than one virtual cluster.

For time synchronization, SYNC messages use relative rather than absolute timestamps to minimize clock drifts. S-MAC also uses a significantly longer listen period than possible clock drift. Although this mechanism can tolerate relatively larger clock drifts, neighboring nodes are still required to update each other periodically with their schedules to prevent errors.

In the second - Data Exchange Phase, nodes exchange data packets using CSMA/CA. Interested neighbors attempt to transmit RTS messages and wait for corresponding CTS. After a successful exchange of RTS/CTS, the sender starts a transmission and extends it into the sleeping period if necessary. The nodes do not follow their sleep schedules until they finish their transmissions, thus, increasing the network performance. In addition to avoiding collision, S-MAC tries to reduce overhearing by letting interfering nodes go to sleep mode after they hear an RTS or CTS. Since data packets are usually much longer than control packets, this approach prevents neighboring nodes from overhearing long data packets and the following ACKs.

The periodic wakeup scheme adopted by S-MAC allows nodes to spend a lot of their time in sleep mode. However, this presents a considerable per-hop latency, called sleep latency, especially problematic during high network traffic situations. [11] describes an adaptive-listening scheme, that is if a node overhears its neighbor's RTS/CTS transmission during a listen period, it receives the estimated length of that 
data transmission before going to sleep following its normal schedule. However, the node wakes up for a short period of time at the end of that transmission to check whether it is the next hop of this multihop message. If so, its neighbor immediately passes the data to it after RTS/CTS exchange, thus, eliminating the requirement of a neighbor to wait for the next scheduled listen time of this node and minimizing latency.

Performance analysis of S-MAC shows that it outperforms MAC protocols such as IEEE 802.11 which was designed for ordinary ad-hoc networks. However, S-MAC has some drawbacks:

- Difficulties adapting the length of the wakeup period to changing load situations due to its fixed duty cycle.

- Broadcast data packets do not use RTS/CTS which increases collision probability.

- Adaptive listening incurs overhearing or idle listening if a packet is not destined to the listening node.

T-MAC [53], likewise S-MAC, is a duty cycle protocol but it adaptively shortens the listen period. It reduces idle listening by allowing nodes to transmit all messages in bursts of variable length and sleep between bursts. The length of each burst is determined dynamically. The active time of a node ends prematurely if it does not sense activity on the medium for a specified duration of time. Although TMAC provides better results than S-MAC under variable loads, its aggressive power conserving policy causes nodes to go into a sleep mode earlier than they are supposed to thus, resulting in increased latency and lower throughput. In addition, nodes 
compete for the channel during small periods of activity which degrades performance during high traffic load conditions.

DMAC [12] is a duty cycle protocol aims at achieving low latency, but can still be energy efficient for convergecast traffic applications. In these applications, the data delivery paths from sources to the sink are in a tree structure, a data gathering tree. The sink is the only destination and all nodes except the sink forward any received packets to the next hop.

DMAC assigns wakeup periods to nodes based on a data gathering tree so that nodes on the multihop path wake-up sequentially like in a chain reaction (Figure 2.4 [12]). Each active period consists of two slots: a receive slot and transmit one. During the receive slot, all children of a receiver have transmit slots during which they might compete to get access to the channel. In the transmit slot, a node tries to send a packet to its next hop and receive an ACK. To reduce contention among nodes on the same tree level during the transmit slot, every node backs off for a period $(B P)$ plus random time within a contention window $(C W)$ before packet transmission. After a node receives a packet, it waits for a short period $(S P)$ and then, transmits the ACK back to the sender. DMAC assumes that only a single data packet is transmitted during a transmit slot. Given that, the time of a receive/send slot is set to:

$$
T_{\text {slot }}^{D M A C}=B P+C W+\left[P K T_{\text {length }}+A C K_{\text {length }}\right] \times T_{\text {Byte }}+S P
$$

where $P K T_{\text {length }}$ is the length of a single data packet in bytes, $A C K_{\text {length }}$ is the length of a single ACK in bytes and $T_{B y t e}$ is the time for transmitting one byte over the radio. 


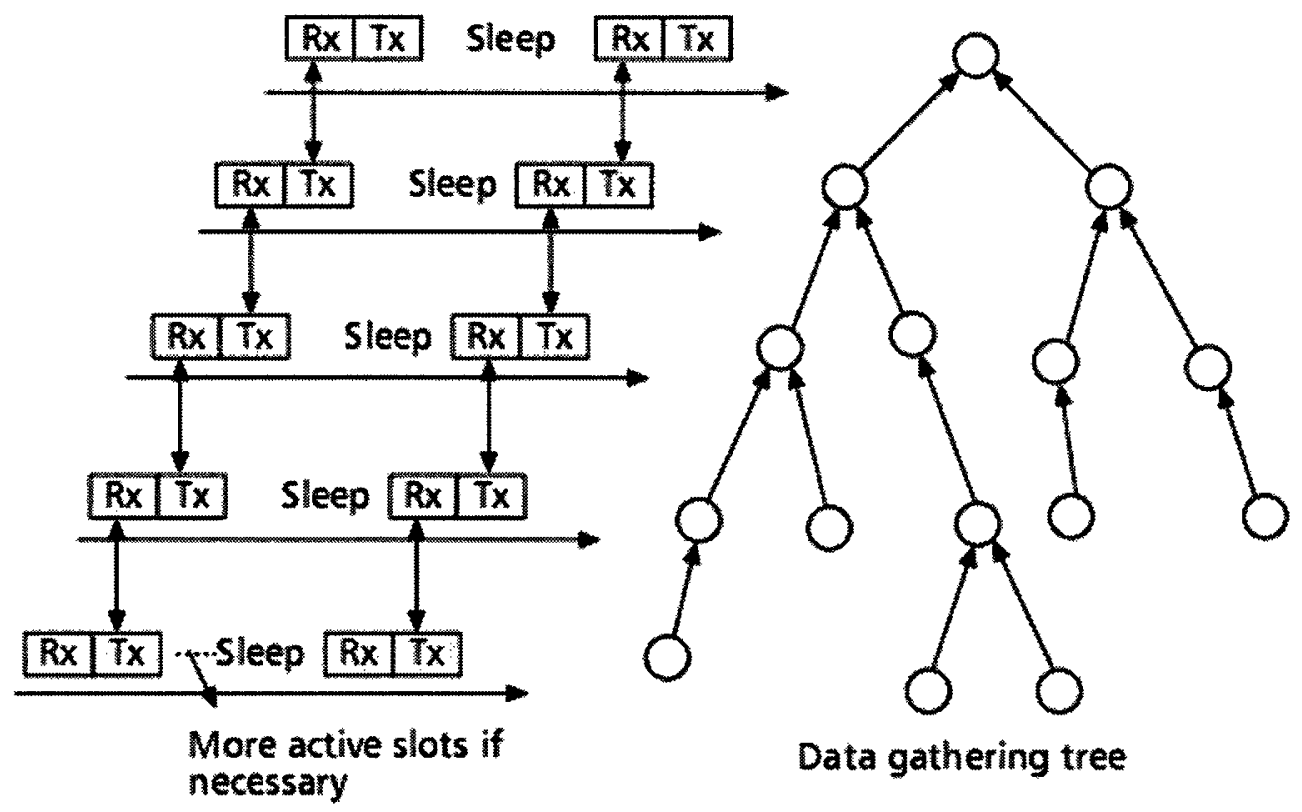

Figure 2.4: A data gathering tree and its DMAC implementation.

DMAC's improvements compared to S-MAC can be summarized as:

- reducing of latency by assigning subsequent slots to nodes that are successive in the data transmission path.

- reducing of contention by separating the nodes' active periods at different levels in the gathering tree.

- avoiding the use of collision avoidance control packets (RTS/CTS) since they would add an extra overhead. However, link level acknowledgements are used to recover lost packets caused by collisions and wireless channel errors.

Despite these improvements, DMAC's performance is still affected by the presence of spatially correlated contention (Figure 2.5) where several close-by nodes detecting the same event, send data almost simultaneously and cause spatially and temporally 
correlated contention. Not employing collision avoidance mechanisms might cause the throughput to degrade as the spatially correlated contention becomes more intense and collisions increase. Subsequently, average end-to-end delay would increase and energy efficiency would decrease. In addition, DMAC does not address two energy waste factors: idle listening and overhearing which could happen during a node's active period.

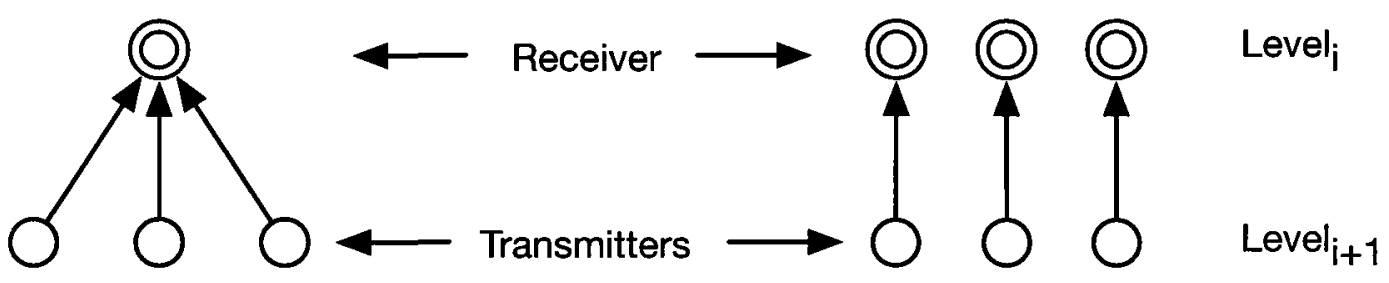

(a)

(b)

Figure 2.5: DMAC spatial contention. (a) In DMAC, all child nodes in Level $_{i+1}$ contend to transmit to the same parent in Level $_{i}$. (b) In S-MAC, child nodes may wake-up at different time intervals to communicate with the same parent.

\section{Wakeup On-demand MAC Protocols}

Unlike duty cycle protocols, Wakeup On-demand protocols do not make attempts to synchronize the schedules of the nodes in the network. Instead, they use different mechanisms to wake-up sleeping nodes only when network activity is present. These mechanisms are grouped into two protocol categories: Two-radio Wake-up protocols and Low-Power listening (LPL) protocols.

The two-radio protocols, e.g. STEM [61], use a two-radio sensor node architecture which consists of a main radio for exchange of data and a wake-up radio which can trigger a main receiver if necessary (on demand) [62] . The wake-up radio uses much less power by either adopting a low duty cycle and/or using a low power hardware. 
STEM $[61,63,64]$ is a two-radio protocol which achieves energy savings by letting the primary radio sleep until communication is necessary, while the wake-up radio periodically listens to the channel following a specified duty cycle. There are two channels used in STEM: the first one - data channel is always in sleep mode except when transmitting or receiving data packets. While the time of the second channel - wakeup channel is divided into fixed-length wakeup periods. Each wakeup period is subdivided into a listen period and sleep period. In the sleep period, the wakeup channel transceiver enters sleep mode; if a node enters the listen period, it simply switches on its receiver for the wakeup channel and waits for incoming signals. If no data is received during the listen period, the node returns to sleep mode. Otherwise the transmitter and receiver start a packet transfer on the data channel.

STEM-B [61], a variant of STEM, periodically transmits beacons on the wakeup channel without prior carrier sensing. The beacon is transmitted on the control channel for one full wakeup period to make sure it hits the receiver's listen period. Each beacon holds the MAC addresses of the transmitter and receiver. As soon as the receiver hears the beacon, it sends an acknowledgment back to the transmitter on the wakeup channel (causing the transmitter to stop beacon transmission) and switches on the transceiver for the data channel. Then, both nodes can proceed to execute the regular CSAM protocol on the data channel. Any other node receiving the beacon on the wakeup channel realizes that the packet is not destined for it and goes back to the sleep mode. The disadvantage of STEM-B is that in order to ensure beacon detection, the listen time of the receiver's wake-up radio in each cycle period should be longer than the transmission time of an ACK plus the synchronization field in the physical layer. This listening period is rather long and increases energy consumption. 
STEM-T [61] uses a busy tone, instead of encoded data as a wake-up signal. Again, the tone is transmitted on the control channel for a time long enough to reach the receiver's listen period. All nodes capable of receiving the tone switch on their data channel for possible incoming data. A node can go back to sleep only when it determines from the packet exchange on the data channel that is not involved in the data transfer. The advantage of the STEM-T is its ability to significantly reduce the listen time in each cycle period since detecting a wake-up tone needs a much shorter time than listening to a beacon in STEM-B.

In general, the advantage of the wakeup radio in STEM is that only the lowpower wakeup transceiver has to be turned on at all times, whereas the significantly higher energy consuming data transceiver is active only if the node is involved in data transmissions. Nevertheless, there are several drawbacks associated with using a wakeup radio: First, requiring nodes to be equipped with an extra low power radio could increase hardware cost and complexity. Second, the range of the wakeup radio and data radios should be the same. If the range of the wakeup radio is shorter than that of the data radio, the possibility of failing to wake-up all neighboring nodes exists.

LPL MAC protocols, unlike the discussed two-radio protocols, use a single RF both for data exchange and waking up neighbors. These protocols eliminate the drawbacks associated with using the two-radio sensor architecture. At the same time, they achieve the very same objective of energy reduction by waking up nodes on demand. Examples of LPL protocols are: Preamble Sampling [67], BMAC [68] and the Wake-Up Frame [69]. 
Preamble Sampling [67] is a basic form of low-power listening in which the RF transceiver periodically wakes itself up for a very short period of time to detect if the channel is busy using a Received Signal Strength Indicator (RSSI). This process is called sampling. To send a data packet to a sampling node, the transmitter first sends a long wake-up- preamble (WUP) with the length of at least one cycle period to wake-up the receiver. The emitted power of the WUP is detected by the sampling nodes using a power-efficient RSSI detector.

B-MAC [68] is another preamble sampling protocol. It achieves energy efficiency by avoiding micro-controller wakeup except if a channel activity is detected. B-MAC utilizes a clear channel assessment (CCA) algorithm looking for outliers in the received signal so that the channel energy is significantly below the noise floor. If an outlier exists during the channel sampling period, the channel is assumed to be clear. After five samples are taken and an outlier is not found, the channel is busy. If activity is detected based on the CCA outcome, a node powers up and stays awake for the time required to receive the incoming packet. After reception, the node returns to the sleep mode. If a packet is not received, a timeout forces the node back to sleep.

Both preamble sampling and B-MAC inherit the same disadvantage of STEM-T, i.e. not only destination but also all other neighboring nodes are woken up generating many overhearers significant power waste while receiving long preambles.

The Wake-Up Frame $[69,70]$ propose an enhanced preamble sampling-based LPL protocol which uses a Wake-Up Frame (WUF) instead of the preamble employed in the preamble sampling protocol. The WUF is composed of multiple short WUFs (SWUFs). Each SWUF includes the destination MAC address and the position of 
the current SWUF in the whole WUF. Upon sampling the medium for a WUF, the receiving node looks for the first SWUF within the WUF. Based on the received destination MAC address field in the SWUF, the node can decide whether it is an overhearer or the destination. If it is an overhearer, it goes to sleep immediately. On the other hand, if it is the destination, it can calculate the start time of the data frame according to the position field so that it can go to sleep until the data comes. Thus, the amount of unnecessary waiting time is reduced. Since the length of the SWUF is on the order of a few milliseconds compared to the hundreds of milliseconds in the basic preamble sampling scheme, the unnecessary waiting time is drastically decreased which saves a large amount of power.

LPL protocols have better energy efficiency because they avoid the long idle listening problem of conventional duty cycle protocols (e.g. S-MAC and T-MAC) when only little network activity is present. However, likewise duty cycle protocols, the use of CSMA channel access causes throughput and energy efficiency degradation due to collisions especially in high contention, high data rate situations. Although collision avoidance (RTS/CTS) could alleviate the hidden terminal problem, it incurs high overhead because data packets in sensor networks are usually very small.

\subsubsection{Hybrid MAC Protocols}

Hybrid MAC protocols attempt to integrate the controllability of centralized protocols with the flexibility of distributed protocols. In addition, these protocols might combine different access schemes (e.g. TDMA and CSMA or TDMA and CDMA) during their operation. Two different hybrid MAC protocols, ZMAC and LEACH are discussed in this section. 
Z-MAC [13] proposes a novel way of combining CSMA and TDMA to adapt to the level of contention in the network. In the low contention level, CSMA is generally adopted, while in the high contention level, TDMA is desired in order to enhance contention resolution. Z-MAC combines the strength of CSMA and TDMA while offsetting their weaknesses. Under low contention modes, CSMA could achieve high channel utilization while under high contention modes, TDMA could perform better in terms of channel utilization.

The Z-MAC operation is divided into three phases: neighbor discovery phase, slot assignment phase and steady state phase. In the neighbor discovery and slot assignment phases, nodes learn about their neighborhood and subsequently, engage in the slot assignment process. During the steady state phase, data exchange might take place.

In the neighbor discovery phase, nodes learn about their single hop and two hop neighbors by periodically broadcasting Hello messages. The collected information is used by Z-MAC as input to its TDMA slot assignment algorithm during the slot assignment phase also called DRAND phase. DRAND [10] is a distributed channelscheduling implementation of RAND [74], a centralized channel reuse scheduling algorithm. DRAND assigns a single slot to each node for transmission while ensuring there is not two nodes within a two-hop communication neighborhood assigned the same slot. Each slot assigned a particular node that node is called an owner and all others nodes are non-owners of that slot. There can be more than one owner per slot since DRAND allows for any two nodes beyond their two-hop neighborhoods to own the same slot. At the end of the DRAND phase, every node forwards its frame size and slot number to its two-hop neighbors. Thus, a node knows about the slot 
and frame information of its one-hop and two-hop neighbors at the beginning of the Z-MAC phase.

During the Z-MAC (steady state) phase, a node can be in one of two modes: low contention level (LCL) or high contention level (HCL). A node is in HCL only when it receives an explicit contention notification (ECN) message from a two-hop neighbor within the last contention notification period. Otherwise, the node is in LCL. A node sends an ECN when it experiences high contention. This contention can be estimated using either of the following:

1. Relate the number of unacknowledged packets to loss rate since both data and ack can be lost due to collisions caused by contention. If the loss rate reaches to a certain threshold, this is a sign of HCL.

2. Measure the noise level of the channel. High contention tends to increase the noise level.

In the LCL mode, any node can compete to transmit in any slot (Z-MAC behaves like CSMA) while in the HCL mode, only the owners of the current slot and their one-hop neighbors are allowed to compete for channel access (Z-MAC enforces the use of TDMA). In both modes, ZMAC uses different backoff window sizes. A backoff window for slot owners is called $T_{o}$ and a backoff window for non-owners is called $T_{n o}$. The sizes of $T_{o}$ and $T_{n o}$ are chosen so that owners have higher access priority to their channel over non-owners. If a slot does not have an owner or its owner does not have data to send, non-owners can use it. This feature achieves high channel utilization even under low contention since a node can transmit as soon as the channel is available. 
The slot duration of Z-MAC must be long enough to transmit one packet with a possible ACK plus an additional time to account for the check interval, CCA period and the maximum contention period. Therefore, Z-MAC slot duration $-T_{\text {slot }}^{Z-M A C}$ is expressed by:

$$
T_{\text {slot }}^{Z-M A C}=T_{\text {check }}+T_{o}+T_{n o}+T_{C A A}+\left[P K T_{\text {length }}+A C K_{\text {length }}\right] \times T_{\text {Byte }}+S P
$$

where $T_{\text {check }}$ is the check interval and $T_{C C A}$ is the CCA period.

The hybrid operation of Z-MAC is mainly intended for achieving an adaptive and robust protocol which can handle dynamic topology changes, varying levels of contention and time synchronization failures. In [13], the authors show that in multihop wireless network, Z-MAC performs better in terms of throughput, latency and channel utilization than other related protocols, namely, B-MAC, Sift (CSMA-based) [75], and PTDMA (hybrid probabilistic TDMA) [76].

To conserve energy, Z-MAC runs on top of B-MAC. Each node maintains a listening duty cycle separated by a check period and each transmission is preceded by a preamble as large as the check period. As discussed in Section 2.3.2, B-MAC has the advantage of reduced energy consumption due to its LPL. Nonetheless, Z-MAC hybrid operation might cause a considerable energy waste because:

1. Each node must periodically perform low power listening due to its lack of knowledge about neighboring nodes sending as well as arrival times (see Figure 2.6). As a result, all nodes usually wake-up in every slot, to check for data transmission to them. High data rate applications require short check interval $T_{\text {check }}$ which means a shorter slot duration. Subsequently, nodes listens to the channel at a higher frequency causing energy waste. Switching the radio ON 
and $\mathrm{OFF}$ is another cause of energy consumption. If during a certain frame, a node has the knowledge about slots in which it would receive neighbor nodes' data and the total number of data bytes, it can be smarter in determining the right slot to listen to the channel. If the node has already collected all the data it needs in this round, it can turn off the radio due to the lack of the data directed to it in this frame.

Frame

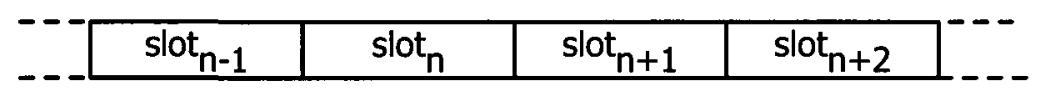

Receiver

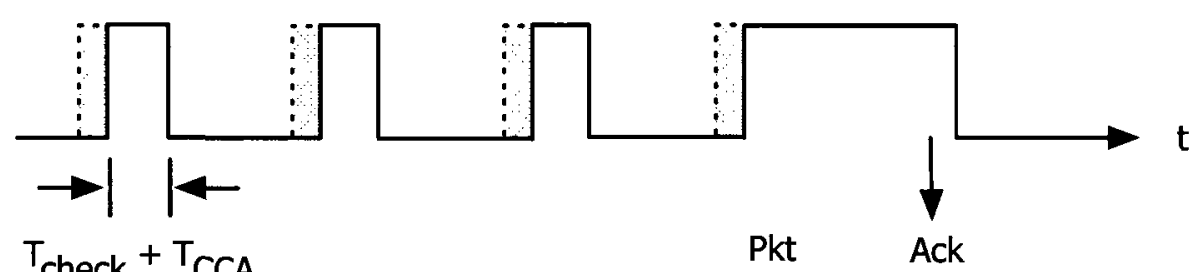

Transmitter
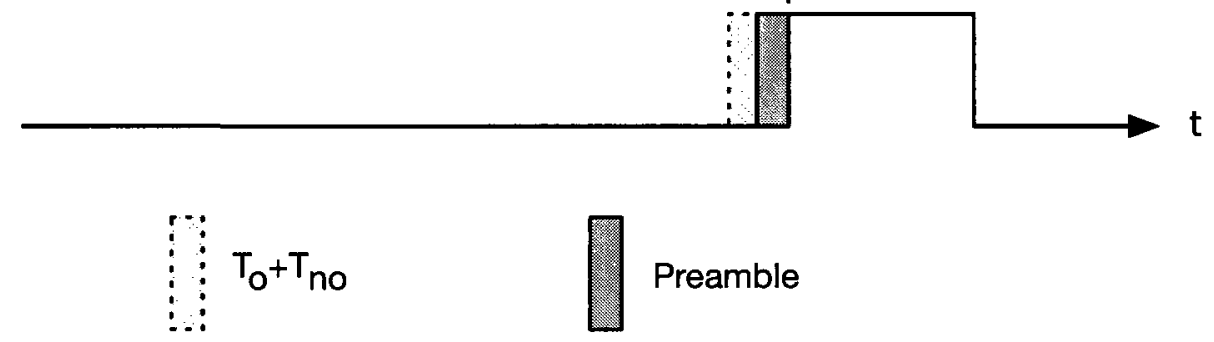

Figure 2.6: Low power listening in Z-MAC

2. If during high traffic, a node has excessive buffered data and the data transfer cannot be completed in its owner slot, the node will contend for the slots of other nodes. Z-MAC uses ECN to minimize this contention at the expense of additional overhead. In addition, ECN messages themselves are susceptible to collisions or loss due to channel errors. 
LEACH [51] is a hybrid TDMA/CDMA MAC protocol integrated with distributed clustering and a simple routing protocol. Localized coordination among sensors is used to improve scalability and robustness. In addition, it incorporates data fusion into the routing protocol in order to reduce the amount of information that must be transmitted to the base station.

LEACH divides the network into several clusters, each cluster has a dedicated node called the cluster-head, responsible for creating and maintaining a TDMA schedule. All the other nodes of a cluster are member nodes. During their assigned time slots, nodes are in active state in order to exchange data with their cluster-head; the rest of the time they are in sleep state. The cluster-head aggregates the data of its members and transmits it to the sink or to other nodes for further relaying. The clusterhead's role is energy consuming since it is always switched on and responsible for data aggregation as well as long-range transmissions. Allowing a fixed node to be the cluster-head burns that nodes' energy resources, after its death, its members would not be able communicate their data to the sink. To solve this problem, adaptive clustering, i.e. re-clustering after a given interval with a randomized rotation of the energy-constrained cluster head to ensure uniform energy dissipation in network, is proposed [51].

The operation of LEACH is organized in rounds and each round is subdivided into two phases: a setup phase and a steady state phase. In the setup phase, the clusters are organized and cluster heads are selected. In the steady state phase, the actual data transfer to the base station takes place.

During the setup phase, each node independently of other ones decides whether it would becomes a cluster-head based on the last time it has this role. A node that has 
not been a cluster-head for a long time is more likely to elect itself than one which served such just recently. After the cluster heads have been elected, they broadcast an advertisement message, using CSMA, to the rest of the nodes in the network notifying them of their role as new cluster heads. Upon receiving this advertisement, all non-cluster head nodes choose a cluster to belong to based on the signal strength of the advertisement. The non-cluster head nodes inform the appropriate cluster heads, again using CSMA, of their belonging to the cluster.

After the cluster setup-phase, the cluster-head knows the number of members and their identifiers. It constructs a TDMA schedule, assigns a time slot to each node and broadcasts the schedule to all the nodes in the cluster. In addition, it picks a random CDMA code to reduce interference from nodes belonging to other clusters. During the steady state phase, the sensor nodes can begin sensing and transmitting data to the cluster heads. The cluster head node aggregates all received data before sending them to the base station. After a certain time, determined a priori, the network goes back into the setup phase and enters another round of new cluster head selection.

There are three main disadvantages to $\mathrm{LEACH}$ :

1. Cluster formation and restructuring can take a long time during which the sensor nodes consume energy and cannot perform any useful work.

2. The requirement that nodes directly communicate with the base station would cause nodes to consume large amounts of energy. This drawback is addressed by forming a routing structure out of the cluster-heads or performing a hierarchical structure of clusters.

3. Uniform distribution of cluster-heads is not easily achieved. Since cluster-heads 
might be concentrated in one part of the network, there would be nodes without any cluster head in their vicinity. This problem is solved by proposing LEACH-C [51] which can conserve more energy by dispersing the cluster heads throughout the network using location information. Support for localization would increase the node cost and power consumption for either hardware support (e.g. GPS) or protocol support (range estimation algorithms).

Despite these drawbacks, for sensing applications with highly correlated, constantly streamed data, LEACH can operate with low energy consumption and extend the network lifetime [51].

\subsection{Multihop Scheduling in Sensor Networks}

Scheduling communication in multihop networks while optimizing a certain objective function is a challenging task because in such networks spatial reuse of a time slot might be possible, i.e. more than one node can transmit at the same slot if their receivers are mutually outside the range of the other node's sender. In the context of multihop TDMA, the problem about scheduling is to determine the smallest length conflict-free assignment of slots where each link or node is activated at least once [79]. In graph theory, minimum slot link scheduling can be represented as an edge coloring problem where the objective is to find the minimum number of colors (time slots) which can be used to color each edge so that adjacent edges receive distinct colors. Edges with the same color can be scheduled simultaneously. As shown in [79], [80], [81] and [82] optimal edge coloring is an NP-complete optimization problem. Therefore, approximation techniques are used to achieve a suboptimal solution. 
For ad-hoc and sensor networks, a number of graph-based scheduling schemes and edge coloring heuristics have been proposed. For example, DRAND [10], a distributed randomized time slot assignment algorithm, finds a time slot for each node, given an input graph and conflict definition so that if any two nodes are in conflict they do not have the same time slot. DRAND runs in rounds and during each round a node may negotiate with its neighbors the selection of a time slot by exchanging a number of messages (request, grant, reject, fail, release). To choose a new time slot, a node broadcasts a request to its one-hop neighbors. If a neighboring node is already processing another request it sends back a reject message. Upon receiving the reject message, the node broadcasts a fail message and start over gain. As the node receives grant messages from its one-hop neighbors, it decides on its time slot to be the minimum of the time slots that have not been taken by its two-hop neighbors prior to this round (this information is piggy-backed in the grant messages). Then, the node broadcasts a release message containing information about its selected time slot to its one-hop neighbors.

Other work by [80] proposes three algorithms based on the coloring method in graph theory with the objective of minimizing data collection time. [83] proposes a distributed edge-coloring algorithm.

An example of optimization-based scheduling is given in [84] where a convex optimization model is formulated to study the tradeoff between the total energy consumption and delay of a link scheduling algorithm which finds the minimum-delay in transferring a fixed number of bits from the source nodes to the sink. On the other hand, [85] formulates a non-linear optimization problem to compute lifetime-optimal routing flow, link schedule and link transmission powers for all active time slots. A 
mixed integer-convex program is used to produce optimal results for interference-free time TDMA schedules. The purpose of [86] is to save energy by minimizing the number of state transitions between active and sleep modes where a Tabu Search based technique is used. A different stochastic multiobjective optimization technique by [87] is based on Particle Swarm Optimization (PSO) and a genetic algorithm (GA). The desired objectives are to minimize the total time for collecting a set of data and save the energy.

\subsection{Summary}

In this chapter, previous works related to the proposed approaches in this dissertation are reviewed. Specifically, previous studies on the topics of: (a) MAC layer design for WSNs, (b) Energy consumption minimization mechanisms for WSNs and (c) multihop scheduling for ad-hoc and WSNs were discussed.

In the area of WSN MAC layer design, protocols are classified into centralized, distributed and hybrid. While centralized MAC protocols relay on a central coordinator for channel access, distributed protocols relay on random channel access. The advantage of distributed protocols over the centralized ones is that the former avoids the overhead associated with coordinating channel access. While hybrid protocols try to combine the advantages of centralized and distributed protocols.

Several energy consumption minimization techniques show that there is not single universal solution for all sensor applications. The discussed mechanisms are:

1. Duty cycling - allows nodes to follow a sleep schedule so that upon waking up a node may participate in data transmission during the wakeup period. Duty cycling is an excellent energy saving mechanism because it gives nodes 
the opportunity to sleep for long periods. However, the longer nodes sleep, the higher delay. In addition, the duty cycle is not adaptive to variable network load.

2. On-demand Wakeup Radio is used for nodes equipped with two radios. The first radio is for data exchange and the other one for triggering a sleeping neighbor. Energy consumption is minimized by putting the more energy consuming radio, i.e. the data exchange radio, to sleep and activating the other radio on a regular basis. The main drawback is the cost associated with having two radios.

3. Low Power Listening is a duty cycling mechanism. However, when a node wakes up, it stays awake for a period long enough to detect channel activity. This period is very short compared to that of the basic duty cycling discussed above. In addition, sensor components other than the radio, e.g. the MCU, are activated only if channel activity is detected, hence, it saves energy.

In the area of multihop scheduling, the problem is to find a conflict-free slot assignment where each link or node is activated at least once. Several solutions based on graph theory have been presented. Other solutions are optimized for certain objective, e.g. delay and/or energy, etc. 


\section{Chapter 3}

\section{On-demand Convergecast}

\section{Scheduling-based MAC}

\subsection{Introduction}

Duty cycle and On-demand Wakeup MAC protocols (Chapter 2) owe in part their performance gain to the use of CSMA as underlying access mechanism. However, for the same reason, they might experience performance degradation under different network operating conditions, for e.g. high contention and/or high traffic rate. On the other hand, a TDMA-based MAC protocol where each node is assigned specific time slots to send, receive data or sleep, inherits the advantage of energy conservation due to the existence of the built-in duty cycle. Furthermore, it can avoid the hidden terminal problem without extra message overhead (RTS/CTS) by scheduling transmission of interfering nodes at different times.

Nevertheless, a conventional TDMA protocol has a number of drawbacks which make it undesirable for certain sensor applications and/or network configurations. 
First, tasks of slot allocation and schedule maintenance could be complex. Second, it is difficult to change frame size and slot assignment when introducing new nodes. In addition, following a rigid transmission schedule does not allow for effective avoidance of idle listening. Third, TDMA needs clock synchronization; while it is an essential feature of many sensor applications, tight synchronization incurs high energy overhead because of its frequent message exchanges. Fourth, during low contention, TDMA has much lower channel utilization and longer delays than CSMA. Nodes in TDMA can transmit only during their scheduled time slots whereas in CSMA, they can transmit at any time as long as there is not contention.

Despite these drawbacks, we believe that a TDMA solution, tailored to tackle these problems, would eventually achieve a performance gain that outweighs these drawbacks. In addition, it is desirable to integrate various energy efficient mechanisms (e.g. low power listening and on-demand wake-up).

This work, proposes a centralized multihop scheduling TDMA MAC protocol called OCSMACS which supports data collection (source-driven), event-driven and query driven WSN applications with the objective of energy efficiency and delay guarantee. The use of TDMA for these applications is advocated because:

1. Many-to-one communication suggests the presence of $\operatorname{sink}(s)$ in the network. These sinks might have an unlimited (high) power supply and are capable of reaching every node in the network. Network wide transmissions by the sink make synchronization easier to achieve.

2. A periodic network discovery phase makes it possible to introduce large number of new nodes to the network in addition to the possibility of modifying frame size and length. 
3. Most sensor applications assume static deployment where nodes' movement occurs when nodes die (as if those nodes are no longer in their last location) which happens infrequently.

4. An integrated on-demand wakeup and LPL mechanisms can be used to avoid long idle listening so that nodes use their slots only at times of network activity. In addition, nodes in receive mode (receive slot) have the capability to switch to sleep mode if there is no traffic received within certain time period.

5. A multihop TDMA schedule could be designed to: maximize network life time; route data from sensor nodes to the sink in a shorter period of time, or facilitate data aggregation and redundancy reduction; and/or a combination of any of these objectives.

Closely related to centralized multihop scheduling is network topology discovery. Distributed scheduling and topology discovery algorithms, similar to the one adopted in DRNAD and Z-MAC, rely on CSMA to exchange a large number of control messages (e.g. hello messages). In large and dense sensor networks high contention and, subsequently, large number of collisions, might result from nodes attempting to transmit control messages at the same time. If the discovery (or set-up) phase runs for a short period of time, then some nodes might end up being not assigned a transmission slot. On the other hand, having a very long discovery phase is not desirable for two reasons: first, long periods means more control messages, hence more energy is wasted in transmitting and receiving these messages. Second, nodes able to obtain transmission slots early in the setup phase must remain active, mostly idle listening, in order to share their current status with other requesting nodes. Likewise, nodes which find the medium busy most of the time or lost their control messages remain 
active (once again idle listening) and retry a number of times before they give up. Long periods of idle listening is a major source of energy waste in sensor networks.

Therefore, an energy-efficient progressive topology construction protocol, called PROGRESSIVE, in which gradual topology information reaches the sink at the same time as the TDMA slots are assigned to already discovered nodes is proposed.

\subsection{Models and Assumptions}

\subsubsection{Network Model}

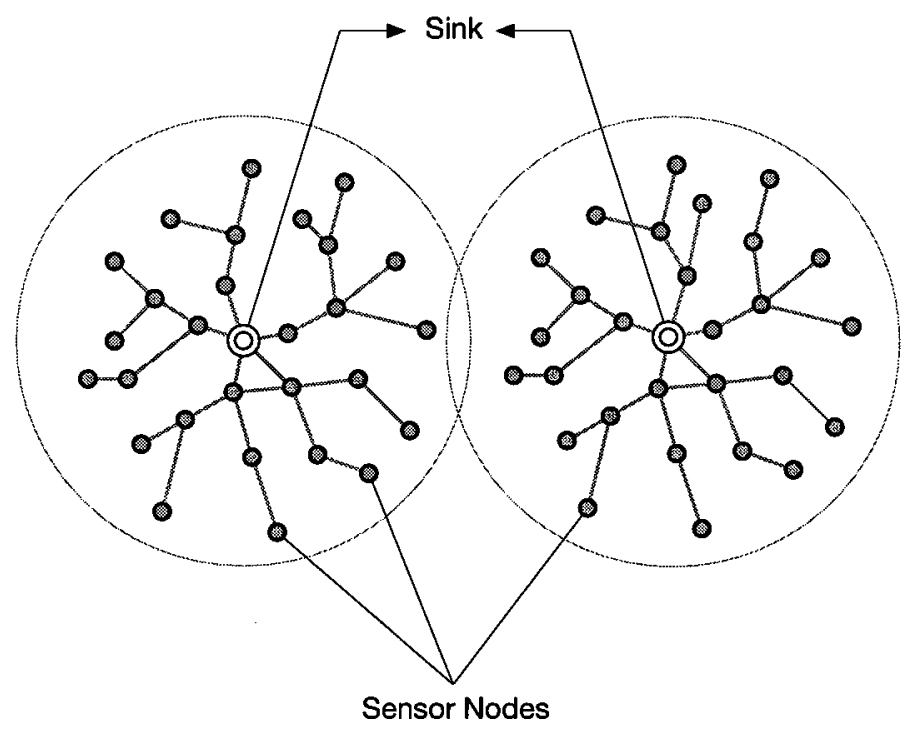

Figure 3.1: WSN model.

The sensor network is viewed as an ad-hoc network of $n$ stationary sensor nodes $N_{1}, N_{2}, \ldots, N_{n}$ of density $\delta_{s n}$ (sensors per unit area) uniformly distributed in a two dimensional field (see Figure 3.1 above). Each node has a receiver, transmitter and a battery with limited energy $E_{s n}$. A node can be either a sender or a receiver but not both at the same time. All nodes are assumed to have omnidirectional antenna which 
transmits at maximum range $\mathcal{R}_{s n}$ using maximum power level, $\mathcal{P}_{s n}$. To be an eligible receiver, a node should be within the transmission range of a sender. A sink is placed in the center of the coverage area and has the ability to transmit at a power level $\mathcal{P}_{\text {sink }}$ $\gg \mathcal{P}_{s n}$ and, therefore, its maximum range would be $\mathcal{R}_{s i n k} \gg \mathcal{R}_{s n}$. This transmission range enables the sink to reach all nodes in one hop; however, the path from a node back to the sink includes several hops. The sink and all nodes associated with it, is considered to form a cell or a cluster. The sink is the entity which receives data from sensors within the same cluster; it is also responsible for time synchronization, and schedule creation and transmission.

\subsubsection{Channel Model}

The communication medium is assumed to be shared by all nodes including the sink. If a receiver is within the range of multiple senders, interference might occur when more than one sender attempts to transmit simultaneously. However, there would not be interference if two receivers are mutually outside the range of other sender.

When node $N_{i}$ transmits with power $\mathcal{P}_{s n}$, node $N_{j}$ receives the transmitted signal with power $G_{i j} \mathcal{P}_{s n}$, where $G_{i j}=K d_{i j}^{-\alpha} . K$ is a constant, same for all nodes; $d_{i j}$ is the distance between the nodes $N_{i}$ and $N_{j}$ and $\alpha \geq 2$ is the path loss exponent.

Let $N_{t}: t \in \mathcal{T}$ be the set of transmitting nodes at a given time with each node transmitting with power $\mathcal{P}_{s n}$. Let us assume that node $N_{j}, j \notin \mathcal{T}$ is receiving a data packet from $N_{i}, i \in \mathcal{T}$. Then, the SNR at node $N_{j}$ would be:

$$
\gamma_{j}=\frac{G_{i j} \mathcal{P}_{s n}}{\eta+\sum_{k \in \mathcal{T}, k \neq i} G_{k j} \mathcal{P}_{s n}}
$$

where $\eta$ is the thermal noise and background interference power at the receiver. $\eta$ 
is considered to be the same for all nodes. The transmission of the packet would be successful if and only if $\gamma_{j}>\beta$. The latter is a given threshold.

\subsubsection{Network Synchronization}

The $\operatorname{sink}(\mathrm{s})$ utilize(s) the local clock to synchronize sensors in the network. Sensor synchronization takes the form of broadcast messages - Beacons which contain the sink's time stamp. Since one and the same Beacon is used to synchronize all nodes, the opportunity for an error in synchronization from the delay between time-stamping and sending a packet at the transmitter is eliminated. Upon receiving a Beacon, each sensor resets its own clock to the value set in the Beacon. For highly precise timebased network operations, the sink has to account for message propagation delay in the clock adjustment. Since the range of the sink is in the range of hundreds of meters, the propagation delay (a few $\mu \mathrm{sec}$ ) is negligible. The other factor to be taken into consideration is the accuracy required by the TDMA schedule as well as that required by the sensor application since an accumulative clock drift could cause the timing of slots and events to be inaccurate. Typical clock drift of a sensor node in MICA2 platform is up to $40 \mu \mathrm{sec}$ per second.

There are two ways to handle synchronization errors. The first one is to resynchronize nodes before maximum clock drift is reached. For a MICA2 platform, it would require continuous re-synchronization with a period of less than one second to keep the error in the microsecond range, which is a significant overhead in terms of bandwidth and energy consumption. The second solution is to introduce a guard time. It is a precautionary measure used to tolerate the difference in clock readings of communicating nodes at each slot. Increasing guard time requires a sensor node to be 
active mode earlier than its reception time in order to tolerate clock drift. Therefore a trade-off between the guard-time and the frequency of resynchronization is utilized.

\subsection{PROGRESSIVE}

PROGRESSIVE [93] is considered as a network setup phase that precedes OCSMACS steady state operation. PROGRESSIVE consists of three sub-phase: neighbor discovery, topology collection and construction, and progressive adjustment. Figure 3.2 shows these phases and their order during network operations. The following sub-sections describe each sub-phase in detail.

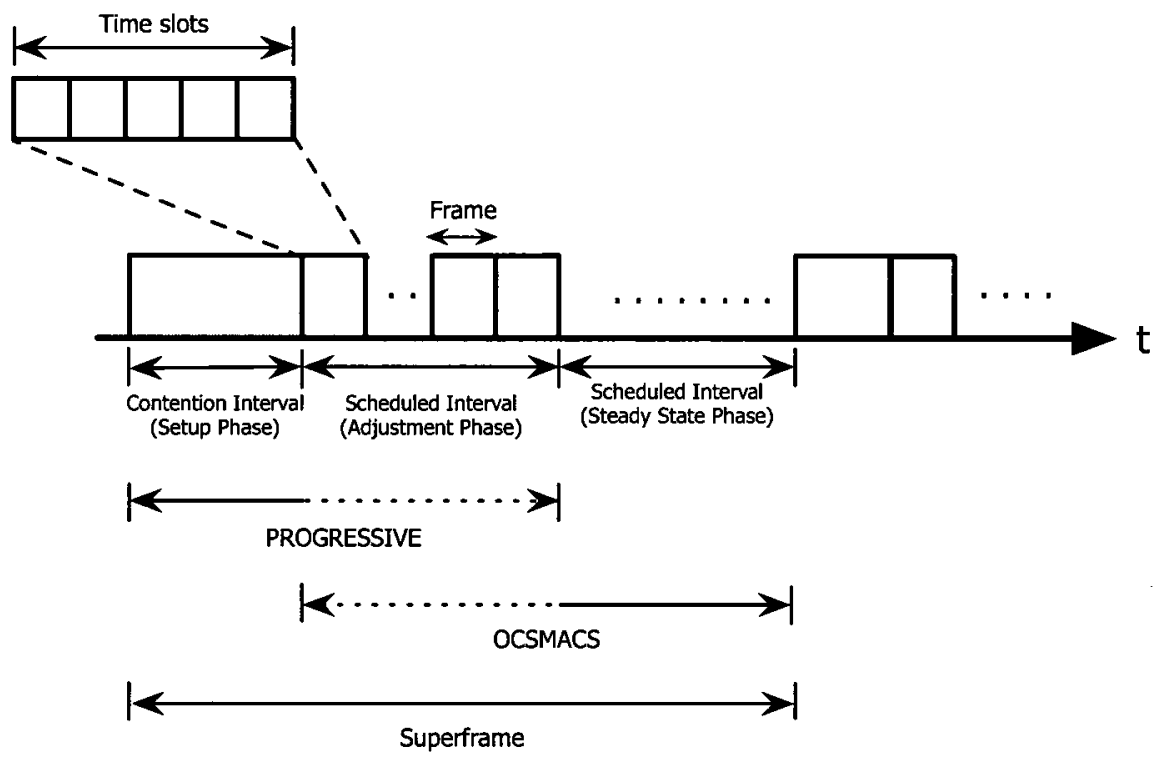

Figure 3.2: OCSMAC Frame structure and phases of operation.

\subsubsection{Neighbor Discovery}

During this phase nodes try to discover their neighboring nodes and their relative location (hop count) with respect to the sink and convey that information to that 
sink. This phase can be also used to provide link quality information.

Nodes independently exchange HeLlo messages, using CSMA, with their neighbors. Each node tries to send a Hello message, senses the medium and if it is idle, the node transmits. If the medium is busy, the node waits for a random time $\left(t_{\text {nextHello }}\right)$ and tries again. If the transmission is successful, the node waits for another random $t_{\text {nextHello }}$ before transmitting the next HeLLO. The maximum number of message transmissions is proportional to the discovery duration $t_{\text {discovery }}$.

Each Hello carries the node ID and current hop count which is initially set to infinity. Only single hop nodes receive a REPLY message from the sink (which indicates that they are single hop nodes). Each node learns of a neighbor with a better hop count to the sink updates its own including this information in the next HELLO transmission, and so on. Eventually, almost every node has updated information of its hop count as well as those of its neighbors. Setting $t_{\text {discovery }}$ is important to enable nodes to learn more about their neighborhood and detect interfering nodes. Setting a large value could be desirable; however, that would mean more transmissions, possibly more collisions and higher energy consumption. The effect of different $t_{\text {discovery }}$ on the number of detected neighbors during the discovery phase will be studied through simulation.

\subsubsection{Topology Learning and Collection}

The topology collection and construction sub-phase starts by broadcasting a new type of messages called NET-LIST which contain neighborhood information (both about discovered neighbors and interferers). To reduce synchronized broadcasting of neighboring nodes and facilitate the propagation of NET-LIST towards the sink, a backoff 
window - $B W_{N L I S T}$ - is set independently at each node so that nodes closer to the sink have a higher probability of transmitting their NET-LIST before their parents.

$$
B W_{N L I S T}(n)=\operatorname{Random}(R)+R \times(h o p(n)-1)
$$

where $\operatorname{Random}(R)$ returns a random integer in $[0, R]$ and $h o p(n)$ is the hop count of a node $n$.

Figure 3.3 shows an example of the range of $B W_{N L I S T}$ for nodes at different hops when $R$ is 16 . It shows that a node in the first hop can have a backoff counter between 0 and 16 while a node in two hops can have a counter in the $[16,32]$ range. This ensures that nodes in $h o p(n)$ are very likely to transmit before nodes in $h o p(n+1)$. Each node receives a NET-LisT will forward it if two conditions in the received message are satisfied: first, if it contains information about nodes different from those in the receiving node's list. Second, if the transmitting node's hop count is not smaller than that of the receiving one. These two conditions minimize the number of unnecessary transmissions and reduce the overall energy consumed during the process.

\subsubsection{Adjustment}

By the end of the network discovery phase, the sink has a rough picture about the current topology and network connectivity. The accuracy of this information depends on successful transmissions of both HELLO and NET-LIST messages. Using CSMA to transmit these messages causes collisions which increase in dense networks. This also increases the number of nodes which might not have full and/or accurate information about their neighbors. As a result, the sink allocates time slots to a fraction of nodes in the network. 


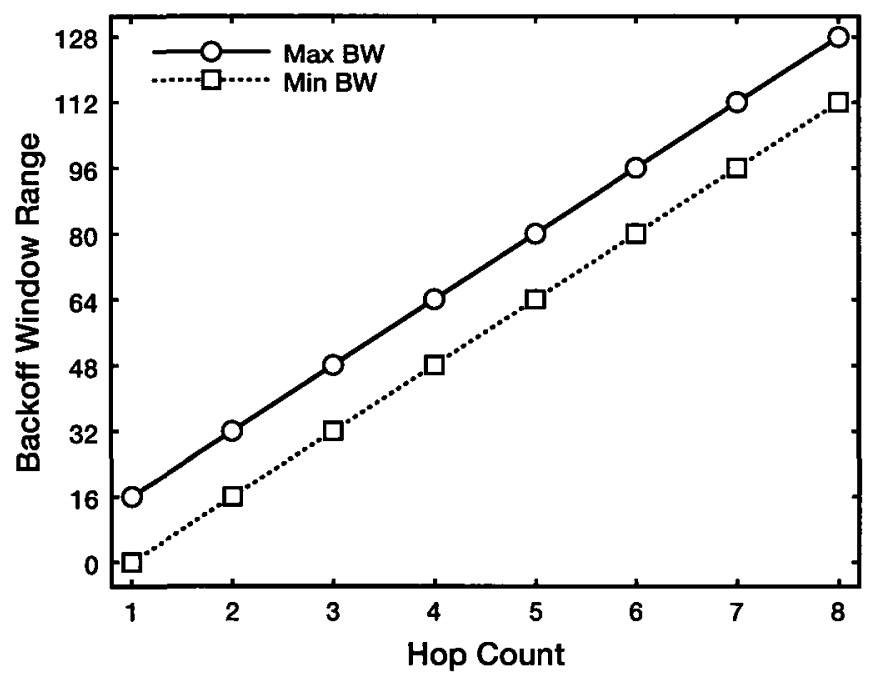

Figure 3.3: Range of $B W_{N L I S T}$ for different hop counts.

The adjustment phase, also called progressive adjustment, allows unscheduled (undiscovered) nodes to notify the sink about their topology information. This phase provides a progressive update to network topology as well as means for resolving scheduling conflicts. It is integrated with OCMACS scheduling phase as follows: once the sink creates a schedule of currently discovered nodes, it broadcasts that schedule as a ScHDL frame. Upon receiving this frame, each node checks a flag called adjustment flag. This flag requires nodes do the following:

- an unscheduled node with a scheduled upstream (parent) to inform the sink about its status.

- if data transmission is taking place, scheduled nodes to defer their data transmissions for a duration of time long enough to allow any unscheduled node or those with scheduling conflicts to inform the sink about their status.

Informing the sink about a scheduling problem is done by sending notification messages (NET-List). At early stage of network operation, it is unlikely that any data 
sampling is taking place. Therefore, time slots could be long enough just to transmit/receive the notification. When a notification message is received, the receiving node checks the list of neighbors included in the message. If its ID is not in the list then, it updates the message by appending its ID. The message is then queued and eventually retransmitted during the upcoming SEND slot. Following notification transmission, a single data packet could be then transmitted.

It is important to highlight that unscheduled nodes intending to send notification messages follow CSMA to minimize the possibility of collisions with other nodes transmitting at the same time slot. Network density and size of the Contention Window ( $C W_{\text {notification }}$ ) affects the number of transmissions required to successfully deliver a notification to the sink. Figure 3.4 is an illustration of a time slot where unscheduled nodes $\mathrm{B}$ and $\mathrm{C}$ compete for the medium to transmit a notification during node A Recieve slot (assuming A has a smaller hop count than the rest).

During the adjustment phase all discovered nodes are scheduled and a ScHDL frame is transmitted as long as new nodes are added based on the new information contained in the notification messages. If the sink does not receive any notification messages during this phase, it assumes that the network is in a stable condition. As a result, with the exception of data exchange periods, further ScHDL frames are not transmitted.

\subsection{OCSMACS}

OCSMACS operation starts once the first node is scheduled using PROGRESSIVE. The initial phase of the OCSMACS overlaps with PROGRESSIVE in the adjustment phase (see Figure 3.2). This overlap is required because while new nodes are 

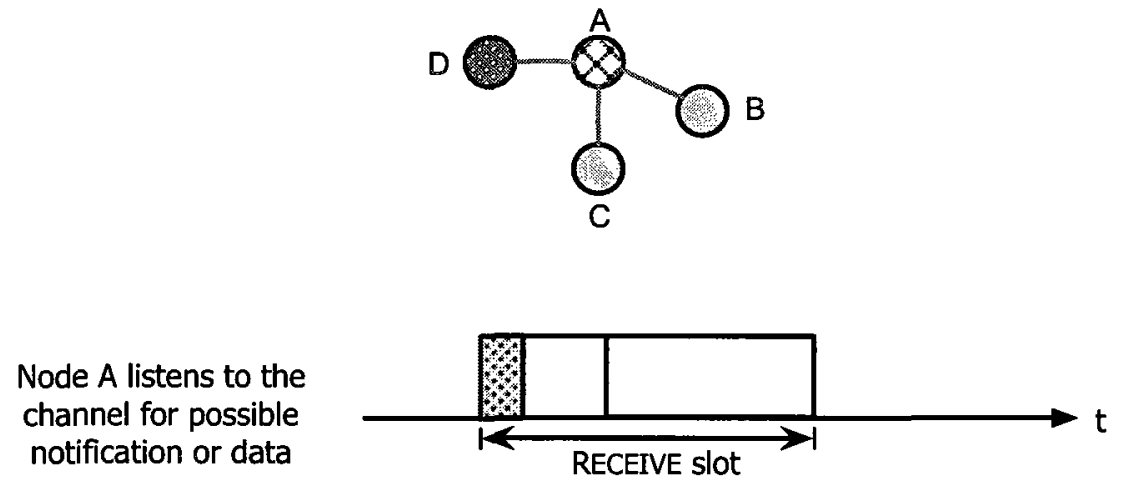

Node $B$ sends notification to node $A$

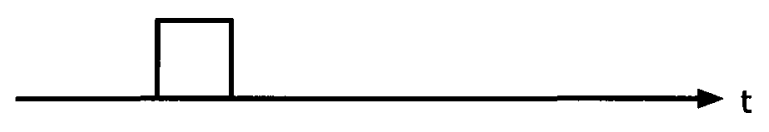

Node $D$ sends data to node $A$

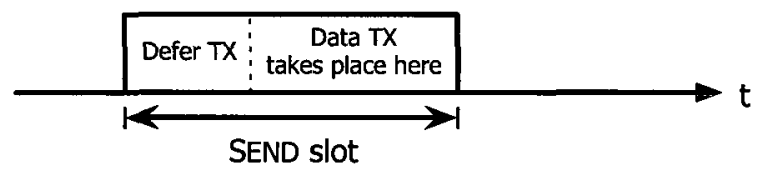
$2 \mathrm{CW}_{\text {notification }}$
- 2 SEND
- Receive
O UNSCHEDULED

Figure 3.4: Topology adjustment. After succeeding in capturing the channel, node B transmits a notification message to node A. Node D starts data transmission only after node B's notification is completely received by node $A$. 
scheduled progressively, those already scheduled would start data transmission using OCSMACS. The figure also shows that the end of OCSMACS's steady state phase marks the end of a superframe and the beginning of a new one as well as the beginning of PROGRESSIVE.

Following the adjustment phase, OCSMACS starts the scheduled steady state phase. The main task of this phase is to provide a highly conflict-free, energy and delay efficient multihop TDMA access for nodes to transmit their sensing data back to the sink. The discussion about OCSMACS includes the frame structure, length and its relation with the specific application requirements (energy and delay). In addition, OCSMACS synchronization, adaptive slot request and assignment will be discussed.

\subsubsection{Frame Structure and Size}

During the course of the steady state phase, nodes might be in one of two types of frames: a sleep frame or an active frame. Each frame has $\mathcal{S}$ data exchange slots. During the sleep frame (see Figure 3.5) all nodes are assigned SLEEP in each data slot. In the active frame, however, nodes can be assigned SEnd, Receive or SLEeP depending on the outcome of the scheduling algorithm which is discussed in Section 3.5 .

The data exchange slot duration depends on the current mode of operation, i.e. whether it is in adjustment phase or steady state scheduled phase. During the adjustment phase, the slot should be long enough to account for $C W_{\text {notification }}, T_{N L I S T}$ and $T_{D A T A}$; while during the steady state scheduled phase, only data transmission is 
allowed. Therefore, OCSMACS's data slot duration - $T_{\text {slot }}^{O C S M A C S}$, is:

$$
T_{\text {slot }}^{\text {OCSMACS }}= \begin{cases}C W_{n o t i f i c a t i o n}+T_{N L I S T}+T_{C C A}+T_{D A T A} & \text { adjustment } \\ T_{C C A}+T_{D A T A} & \text { steady scheduled }\end{cases}
$$

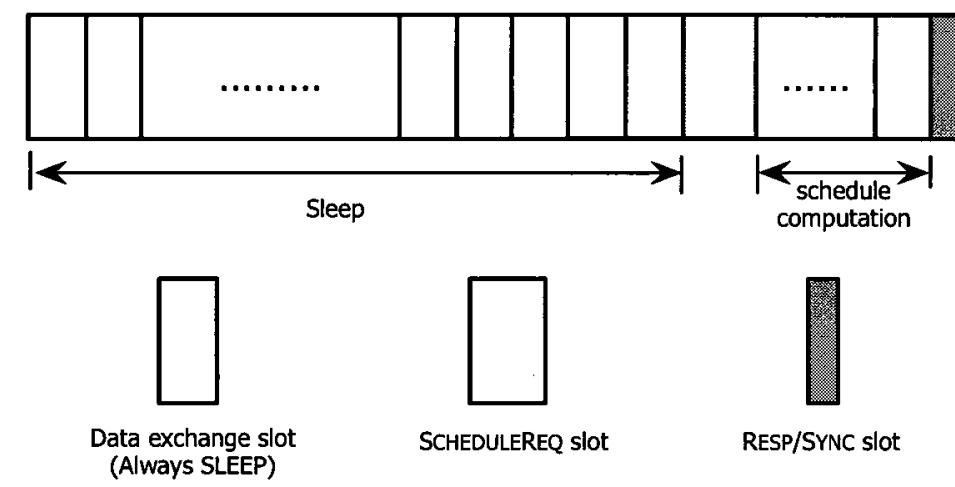

Figure 3.5: Sleep frame structure.

The other two types of slots are ScheduleREQ slot and Resp/Sync slot. These slots are key elements in achieving energy- and delay-efficient design of OCSMACS. There is a single ScheduleREQ slot within each sleep frame. This slot is used as a low power listening mechanism by nodes to transmit and/or relay schedule requests (when sampled data is ready) to the sink. Based on the received node requests, the sink schedules nodes and transmits the new SchdL during the Resp/Sync slot. The time (number of data slots) between the ScheduleREQ and the next Resp/Sync should be long enough for the sink to compute a new schedule. If requests are not received then SCHDL frame is not transmitted and the next frame remains as a sleep frame. This mechanism allows nodes to sleep during the sleep frame and only wake-up for a short period of time during the SCHEDULEREQ slot to sense any traffic request in case there is some or to continue sleeping in case there is none. The other function of the 
Resp/Sync slot is to synchronize nodes by using the SchDL frame or Beacons when there is not network activity, i.e. when SCHDL frame is not transmitted.

Setting the sleep frame size is important for achieving certain application requirements, for instance energy and delay. With respect to energy, nodes sleep time is proportional to frame size, i.e. the bigger the frame size, the longer nodes are able to sleep and hence, conserve energy. However, for event-driven applications with short delay requirements, the longer nodes sleep time translates into longer end-to-end delays. Therefore, the application sampling rate $-r_{\text {sampling }}$ and maximum end-toend delay - delay $y_{\max }$ requirements must be considered in deciding the optimal frame length.

The number of data slots $-\mathcal{S}$ in a frame can be expressed by:

$$
\mathcal{S} \leq\left\lfloor\frac{\text { delay }_{\max }-\left[T_{R E Q}+T_{\text {scheduling }}^{\text {sink }}+T_{S C H D}+T_{\text {multihop }}\right]}{\left.T_{\text {slot }}^{\text {OCSACS }}\right\rfloor}\right\rfloor
$$

s.t.

$$
\begin{gathered}
\text { delay }_{\text {max }}>\left[T_{R E Q}+\right. \\
\frac{\left.T_{\text {scheduling }}^{\text {sink }}+T_{S C H D}+T_{\text {multihop }}\right]}{Q^{\text {max }}}<\frac{1}{r_{\text {sampling }}}
\end{gathered}
$$

where $T_{R E Q}$ is the duration of the SCHEDULEREQ slot, $T_{\text {scheduling }}^{\text {sink }}$ is the time it takes the sink to compute a new schedule, $T_{S C H D}$ is the SCHDL frame transmission time, $T_{\text {multihop }}$ is the expected delay experienced by a packet from the start of its transmission until it reaches the sink and $Q^{\max }$ is the maximum queue size of each node. $T_{\text {frame }}$ is the 
total frame duration and is given by:

$$
T_{\text {frame }}=\mathcal{S} \times T_{\text {data-slot }}^{O C S M A C S}+T_{R E Q}+T_{S C H D}
$$

Equation (3.5) is used in setting an upper bound for the delaymax requirement of the application. If the sum of $T_{R E Q}, T_{\text {multihop }}$ and $T_{\text {scheduling }}^{\text {sink }}$ exceeds delaymax then there is not a solution for equation (3.4). In addition, as per equation (3.6) if the sampling time is less than the sum then packets would be queued at nodes and the end-to-end delay would grow rapidly. Data loss might also occur if queues become full.

\subsubsection{Handling Synchronization}

The number of data slots - $\mathcal{S}$ given in equation (3.4) might need to be adjusted to accommodate synchronization errors. As discussed earlier, these errors could be addressed either by resynchronization using Beacons transmitted during the RESP/SYNC slots, or by using guard time in each slot. The choice of a mechanism depends on the frame size $-T_{\text {frame }}$. For example, Table 3.1 shows maximum clock drifts of MICA2 sensors for different frame sizes.

\begin{tabular}{|l|l|l|l|}
\hline$T_{\text {frame }}(\mathbf{s})$ & max clock drift (ms) & guard time (ms) & overhead (\%) \\
\hline \hline 1 & 0.04 & 0.08 & 0.9 \\
\hline 5 & 0.2 & 0.4 & 4.4 \\
\hline 10 & 0.4 & 0.8 & 8.4 \\
\hline 30 & 1.2 & 2.4 & 21.5 \\
\hline 60 & 2.4 & 4.8 & 35.4 \\
\hline 300 & 12 & 24 & 73.3 \\
\hline 600 & 24 & 48 & 84.6 \\
\hline
\end{tabular}

Table 3.1: Maximum clock drifts of MICA2 sensor for different frame sizes. 
Short frame sizes, e.g. five or ten (s), introduce maximum clock drifts in the order of few hundreds of $\mu$ s. These clock drifts, if added as guard time to each slot, contribute with less than $10 \%$ to the total adjusted slot time (the initial slot time plus the guard time) assuming the initial slot time is $8.75 \mathrm{~ms}$ (the duration of a packet transmission of 42 bytes at $38.4 \mathrm{kbps}$ ). A [4-10] \% overhead which might become an energy waste due to idle listening is still less than the energy waste due to transmitting a Beacon to all nodes at a rate of a Beacon every less than a second.

However, for long frames, e.g. 300s or higher, the required guard time is in the order of tens of ms. This guard time would take more than $50 \%$ of the adjusted slot time and thus increase idle listening and reduce energy efficiency. Therefore, in this case it, is desirable to use a combination of both resynchronization and guard time. Resynchronization is facilitated by adding more RESP/SYNC slots until clock drift drops to an acceptable level. Then, a guard time is added to compensate for clock drifts.

Based on the application sampling time $-r_{\text {sampling }}$, the acceptable level of maximum clock drifts and the maximum clock drift specification of the sensor platform, the number of data exchange slots given by equation (3.4) could be rewritten as:

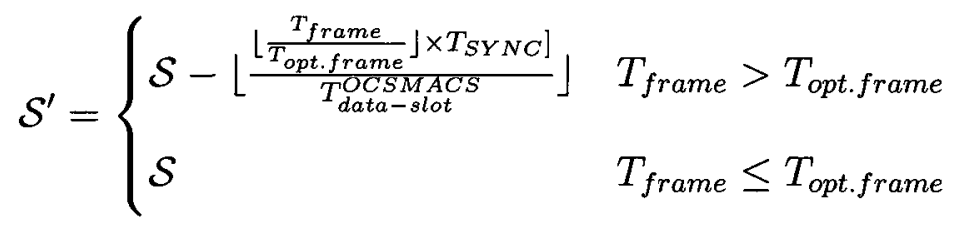

where $r_{\text {opt.frame }}$ is the sampling time which results in an acceptable level of maximum clock drift and thus, a small guard time overhead. $T_{S Y N C}$ is the duration of the RESP/SynC slot. The term $\left\lfloor\frac{T_{\text {frame }}}{T_{\text {opt.frame }}}\right\rfloor$ is the number of additional Beacons which need to be transmitted while maintaining a balance between the guard time overhead 
and the Beacons transmission overhead.

\subsubsection{Requesting Slot Assignment}

The underlying idea to achieving energy efficiency in OCSMACS is that the default mode of operation uses sleep frames. Except during the SchedulEREQ, nodes sleep for the whole frame. Nodes stay active in the ScheduleReq slot to transmit (if a slot is needed) and/or relay schedule requests transmitted by neighbors. For example, if an event is detected or data is sampled as a result of a query, the involved nodes utilize the upcoming ScheduleREQ slot to demand a new schedule during which these nodes are being assigned transmission slots. The new starting frame is called an active frame (Figure 3.6).

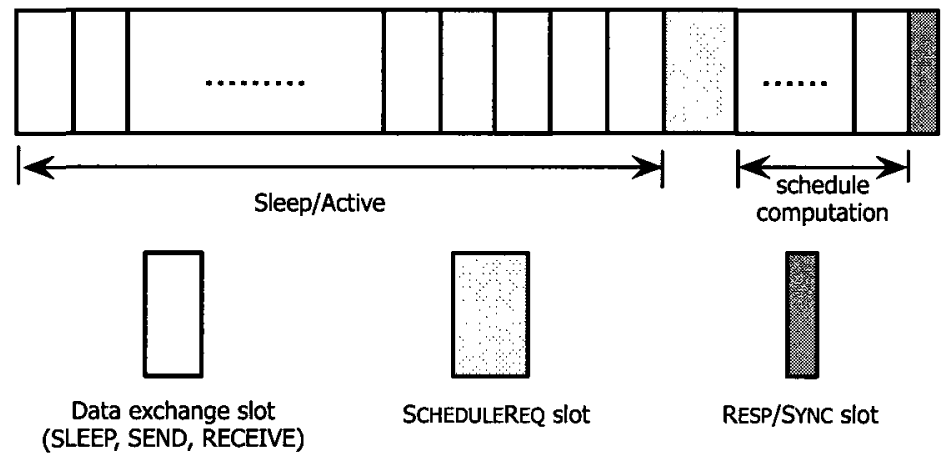

Figure 3.6: The Active frame structure.

A special care must be taken in developing the mechanism to transmit and forward schedule requests to the sink. First, successfully delivering schedule requests to the sink is the only way to guarantee that nodes are able to get slot assignment and, subsequently, communicate their sensing data. Second, schedule requests are overhead that eventually contributes to energy consumption. Third, the duration of the ScheduleREQ slot affects the delay as well as energy consumption. Having a long 
ScheduleReq slot means longer periods of idle listening, especially when data is not sampled which translates into higher energy consumption. In addition, nodes would experience more delay before being able to transmit their data.

To address these issues, a schedule request aggregation process during which multiple schedule requests are combined into a single one is proposed. This process requires dividing the ScheduleReq slot into fixed size request sub-slots. Each sub-slot is long enough to transmit a single schedule request (see Figure 3.7). Moreover, a schedule request aggregation tree, computed by the sink is used to assign a request listen window (a number of request sub-slots) for each node within the ScheduleReQ slot. Listen window assignment information is included in the ScHDL frame transmitted by the sink. During each window a node must listen for schedule request transmissions made by its child nodes. Once the listen window expires, the aggregated schedule requests (including that of the receiving node, if available) are broadcasted in a new message. It has to be noted that there could be more than one parent (upstream node) listening to the same child. Hence, the same schedule request might be routed through out multiple paths before reaching the sink.

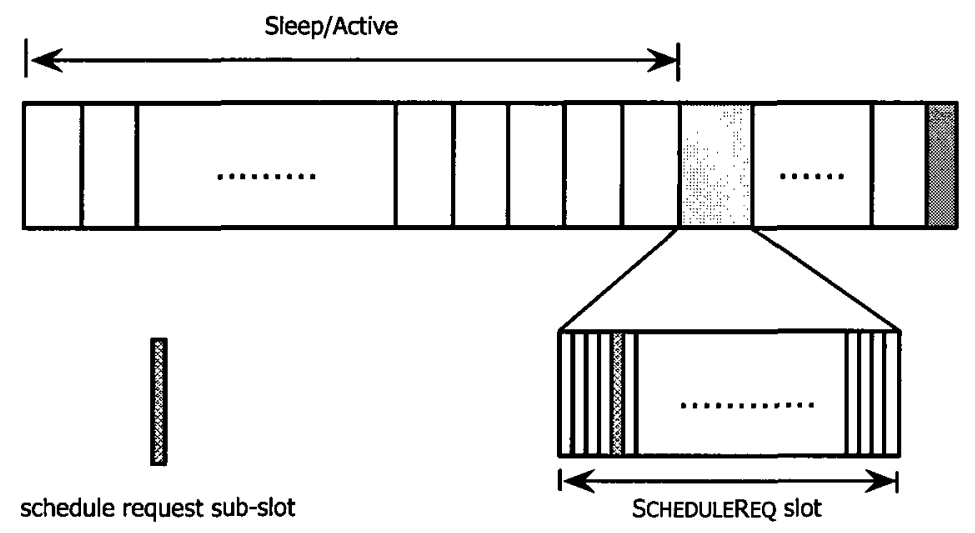

Figure 3.7: Schedule request sub-slots. 
An example of a conventional request aggregation mechanism is concatenating $I D s$ of requesting nodes in a single request message and forwarding it to the next hop. Eventually, the sink would receive schedule requests containing a list of all nodes in need of slot assignment. This mechanism is simple, however, the size of schedule request grows as it gets closer to the sink. Large schedule requests mean longer request sub-slots and, subsequently, longer ScheduleReq slot(s). In large networks, the overhead caused by large schedule requests would negatively affects the energy efficiency of the protocol.

Unlike this one, the proposed mechanism is simple and yet energy-efficient in transmitting many schedule requests in a short period of time. The objective is to control the duration of SCHEDULEREQ slot by using very small fixed-size request messages. The design of these messages is based on the assumption that nodes in close proximity can detect the same event, hence, generate schedule requests in the same period. These nodes will communicate their sensing data almost at the same time. Therefore, only little information about the relative location of this event within the network is sufficient to identifying the active nodes. All unscheduled nodes broadcast their schedule requests during the next SchedULEREQ slot.

Each schedule request message is assumed to have two main fields: activity rootID and activity depth. After receiving a schedule request, the node sets itself as the activity root in two cases. First, if it is a source node and second, if it has received schedule requests from different branches of a tree rooted at itself. Otherwise, it would forward the request message as is. The activity depth is the difference in hop count between the activity root and first initiator of the schedule request. Received schedule requests are stored and aggregated until the schedule listen window expires. 
Then they are forwarded to the next hop. Once a schedule request is received at the sink, the activity root and all nodes within the activity depth from that root are set as active sources. Figure 3.8 provides an example of a network with two detected events. The schedule request aggregation trees show the activity root and activity depth for each event.

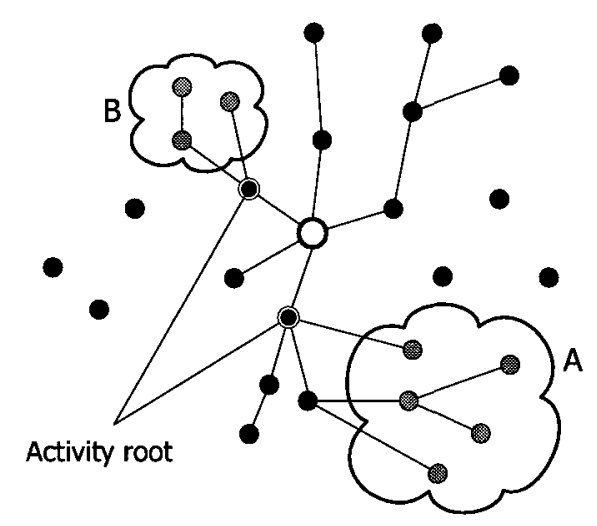

(a)

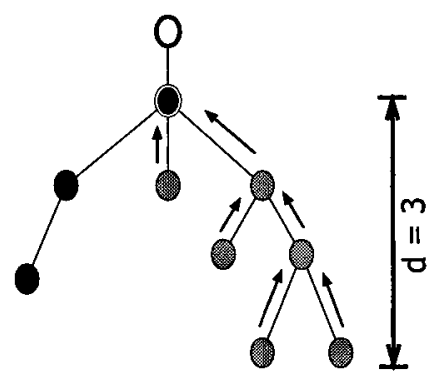

(b)

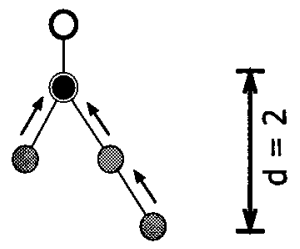

(c)

Figure 3.8: Schedule request. (a) Events A and B are detected in two different parts of a network (b) Request transmission and request aggregation tree for event $\mathrm{A}$ (event depth $=$ 3) (c) Request transmission and request aggregation tree for event $\mathrm{B}$ (event depth $=2$ ).

Despite the advantages this aggregation mechanism offers, it has some drawbacks:

- Inactive sources are unnecessarily selected as members of the aggregation tree.

Figure 3.8 shows two inactive nodes which are in an event $A$ aggregation tree.

- Large number of inactive nodes are redundantly scheduled if sources are scattered throughout the network and the activity root is set on the bases of different schedule requests generated by nodes which are very far, in terms of hop count, from the activity root.

- Schedule requests are unacknowledged, end-to-end delays might increase if requests are lost due to transmission errors. 
The solution to the first problem could reside in associating a timer $-t_{\text {active-source }}(n)$ for each source node $n$. This timer is activated each time a new data packet is received from $n$. However, if data is not received, the timer expires and this particular source is removed from the active sources' list and eventually, would not be assigned any transmission slots in subsequent ScHDl frames. As for the second problems, any node receiving schedule requests from two different nodes would not set itself as the activity root node. Instead, it would forward the schedule request with the larger activity depth and eliminate the other. To minimize delays caused by lost and/or eliminated schedule requests, the number of SCHEDULEREQ slots in the active frame has to be increased.

When the sink receives a request a new slot assignment is computed and transmitted during the next RESP/SynC slot. This SCHDL is broadcasted to all nodes in the network. Upon receiving the ScHDL frame, all nodes store their new slot assignment and reset their timers to start a new active frame. The active frame has identical structure, size and slot duration to that of the sleep frame. However, in an active frame, nodes might be in different states (i.e. send, receive, sleep) at different slots. If a source node does not receive any slot assignment, this means that its schedule request did not go through due to transmission errors or the elimination process discussed above. This node can retransmit its request during the next ScheduleREQ slot.

The computed slot assignment might require a total number of slots greater than the data exchange slots available in a frame. In this case, the computed slot assignment is mapped onto multiple frames as needed. Doing so, does not alter the positions of ScheduleREQ and ReSP/SynC slots in the frame. 


\subsubsection{Joining the Network While Operating}

While the network is in operation, nodes could be added to replace failed ones or improve sensing quality. When a node powers on, it listens to the channel for a Beacon which besides time synchronization provides information about slot duration, the next adjustment phase and the next superframe. Based on which precedes the other, i.e. the adjustment phase or a new superframe, the node performs the following:

- If the adjustment phase is repeated before the end of the current superframe then, the node wakeup during data collection prior to the adjustment phase to learn about existing neighbors. To obtain a transmission slot during the adjustment phase, the node follows the same mechanism described in Section 3.3.3.

- If the adjustment phase is not repeated until the end time of the current superframe, then the node will obtain a slot assignment using PROGRESSIVE scheduling at the beginning of the next superframe.

Figure 3.9 shows the steps which are followed when joining the network while it is in operation. The time it takes a node to join the network (obtain slot assignment) depends on which one of the above cases is followed.

The worst cast delay $-T_{\text {join }}$, can be expressed as:

$$
T_{\text {join }}=\mathcal{F}_{\text {adjustment }} \times T_{\text {frame }}+t_{\text {discovery }}{ }^{1}+t_{\text {adjustment }},
$$

\footnotetext{
${ }^{1}$ Adding $t_{\text {discovery }}$ is applicable only if the next adjustment phase is the beginning of a new superframe.
} 


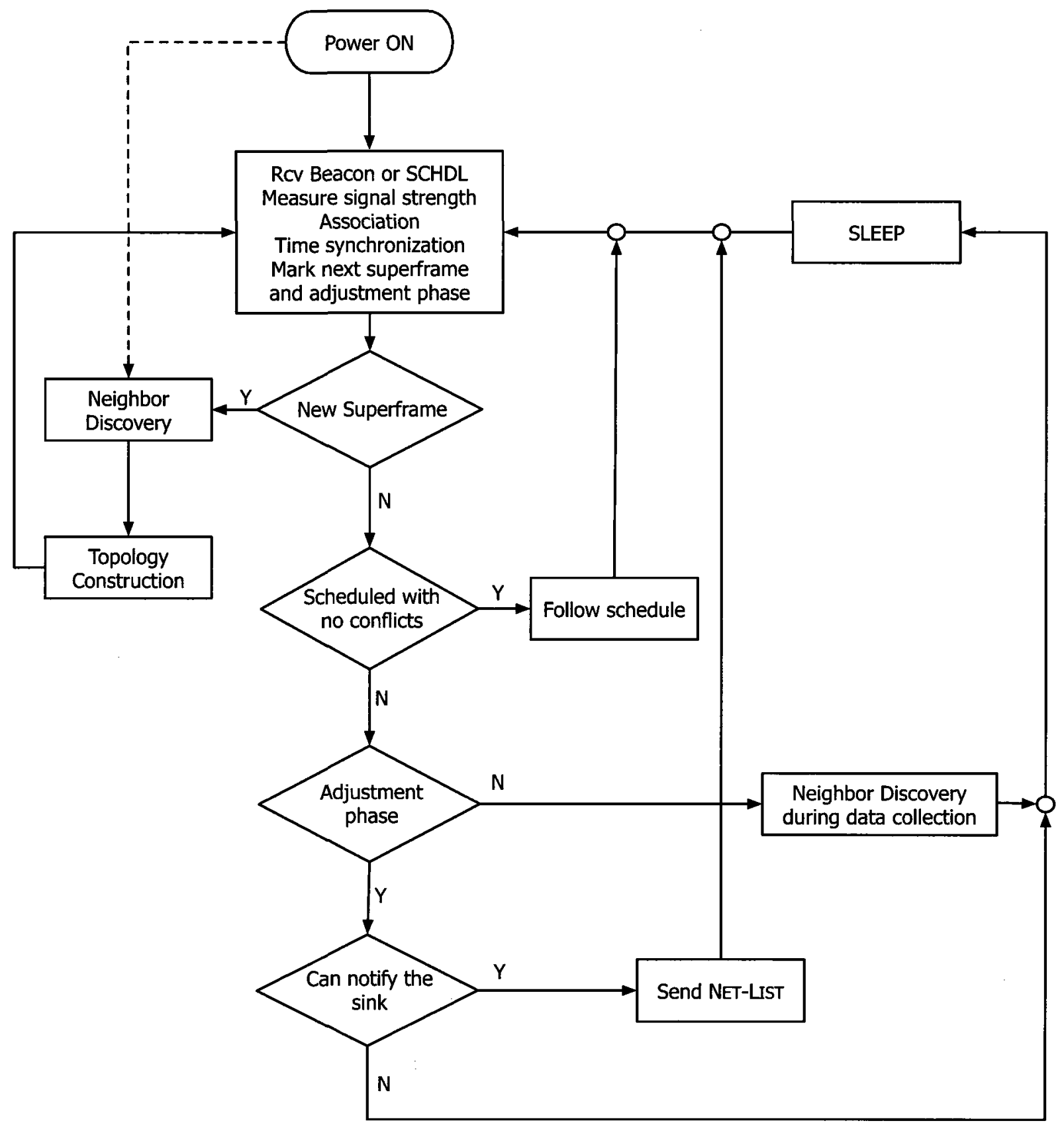

Figure 3.9: Slot assignment while the network in operation. 
where $\mathcal{F}_{\text {adjustment }}$ is the number of frames between two consecutive adjustment phases. $T_{\text {frame }}$ is the frame duration and $t_{\text {adjustment }}$ is the average duration of the adjustment phase; the time until all nodes are assigned time slots. Setting $\mathcal{F}_{\text {adjustment }}$ depends on the frequency of adding new nodes to the network and the desired frequency of refreshing topology information.

\subsection{Multihop scheduling}

A core part of the discussed work is proposing TDMA scheduling protocols for convergecast communications, as data usually flows from sensors towards the sink. Therefore, considering network topology, the main task in designing a TDMA schedule is time slot assignment for every link/node ensuring elimination of collisions and satisfaction of some optimization criteria. Different scheduling algorithms with the main objective of minimizing data collection time while minimizing energy during operation are proposed in this work.

\subsubsection{Scheduling Model and Assumptions}

The network is represented by a graph $G=(V, E) . V$ is the set of nodes including the sink. $N=|V|$ is the number of nodes in $G$. The (undirected) edges $E \in V \times V$ are (transmission) links to be scheduled. An interference graph $C=(V, I)$ so that $I \in V \times V$ is the set of edges and $(u, v) \in I$ if either $u$ or $v$ can interfere with a signal intended for the other. Links which strongly interfere with each other should be scheduled at different time slots to prevent collisions. On the other hand, weakly interfering links can be scheduled simultaneously as long as SNR remains above the 
specified threshold for all receivers in that same slot.

The presented scheduling algorithms use the following set of assumptions and definitions:

1. The time is divided into equivalent time slots. Each time slot $(\tau)$ is long enough to allow a single packet transmission. The same slots can be assigned to different nodes (or links).

2. The network is divided into $K$ hypothetical levels on the basis of hop count so that Level $_{i}$ is $i+1$ hops from the sink, Figure 3.10.

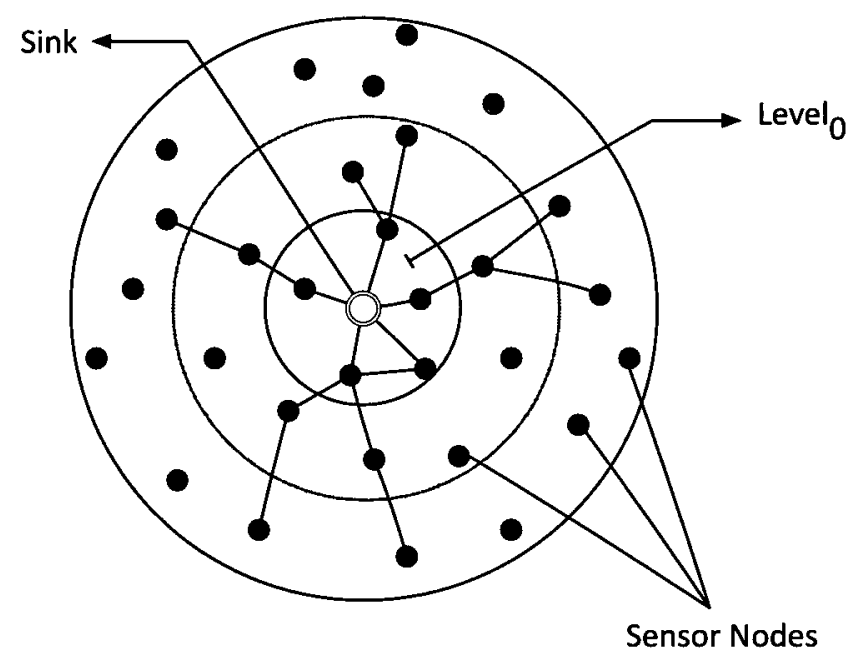

Figure 3.10: Scheduling model view of the WSN.

3. Range $\left(S_{i}, R_{x}\right)$ denotes that node $R_{x}$ (a receiver) is within the transmission range of node $S_{i}$ (a transmitter).

4. $\mathcal{N}\left(S_{i}\right)$ is a list of all neighbors of $S_{i}$ so that $\forall R_{x} \in \mathcal{N}, \operatorname{Range}\left(S_{i}, R_{x}\right)$ is satisfied.

5. tabuReceivers $(\tau)$ is a list of nodes which are not allowed to receive in slot $\tau$. 
6. tabuSenders $(\tau)$ is a list of nodes which are not allowed to send in slot $\tau$.

7. pktsQueued(v) is used to indicate that each node might produce at least one

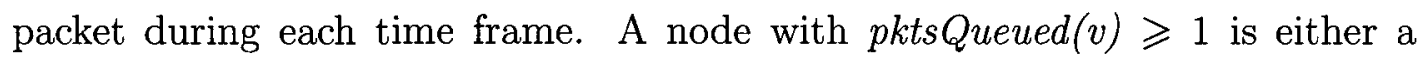
source or relay.

8. receiveCounter $(v)$ is a counter that is incremented each time $v$ is selected as a receiver. Having this information when selecting an upstream receiver the scheduler would choose $v$ with minim receiveCounter $(v)$ to distribute the load among all nodes.

9. $\operatorname{sort}(G$, criterion) is a function which sorts nodes in $G$ based on their hop-count. Criterion is either Top-Down (smallest to largest hop counts) or Bottom-Up (largest to smallest hop-counts).

10. A node can be either a sender, a receiver or in a sleep mode at any given time slot. In addition, a node can switch from send or receive to sleep at any time.

11. A node $R_{x}$ can receive a packet transmitted by a node $S_{i}$ in a time slot $\tau$ only if two condition are satisfied: (1) Range $\left(S_{i}, R_{x}\right)$ and (2) SNR $>\beta_{R_{x}}$.

\subsubsection{Spatial-temporal Scheduling}

Spatial-temporal scheduling [94] assigns time slots for nodes based on their relative distance (hop count) from the sink. In addition, slot assignment is performed taking into consideration many-to-one traffic assumption, i.e. it facilitates the flow of traffic from nodes to the sink.

In spatial-temporal scheduling, the network is divided into different levels based on the number of hops in it. Nodes in Level $_{0}$ are single-hop nodes and nodes in Level 
are nodes in $i+1$ hop. The scheduling process is performed in cycles, each cycle represents a time $\operatorname{slot}^{2}$. During a cycle, nodes in Level $_{i}$ are assigned SEND while their neighbors in Level $_{i-1}$ are assigned Receive. All possible Send-Receive assignments between the two levels are performed while avoiding conflicts. The scheduler keeps track of SNR of the currently assigned receivers. If a node $S_{i}$ is going to be assigned SEND, SNR is updated for all current receivers in this cycle. If the new SNR at receiver $R_{x}$ goes below a specific threshold $\beta_{R_{x}}$ then $S_{i}$ would not be assigned Send in the current cycle; it would also be placed in the tabuSenders $(\tau)$.

Any node assigned Receive must be assigned Send in a later cycle. Nodes in Level $_{0}$ are one hop neighbors of the sink, so the sink is their only receiver. The current cycle is over when further slot assignments are not possible. All remaining unassigned nodes in the current cycle are assigned SLEEP. This slot assignment process is repeated for a number of cycles until all active nodes are assigned at least a single SEND slot. This scheduling slot assignment is called interleaved spatial-temporal scheduling (see A.1.1) since nodes at different levels of the network can be assigned SEND in the same cycle.

Interleaved spatial-temporal scheduling can be performed in two different ways: Bottom-Up (BU) or Top-Down (TD). In Bottom-Up scheduling, each cycle starts slot assignment for nodes in $K-1$ level (nodes with maximum hop count) followed by nodes on the next level closer to the sink, i.e. Level $_{K-2}$, etc. On the other hand, in top-down scheduling each cycle starts slot assignment for nodes in Level $_{0}$, then Level $_{1}$, etc.

Figure 3.11 shows an example of schedules produced by the above discussed algorithms for a simple network of 9 nodes. As demonstrated Top-Down scheduling

\footnotetext{
${ }^{2}$ Cycle and time slot $\tau$ will be used interchangeably within this subsection
} 
generates a smaller frame than that of Bottom-Up scheduling. This is due to the direct proportional dependency between the number of nodes and hop count in uniformly distributed network topology. In other words, there are more nodes in Level $_{i}$ than Level $_{i-1}$. Moreover, Bottom-Up scheduling priorities SEND slot assignment to nodes at higher hop count. As a result, more slots are needed to allow all nodes at higher hop counts to transmit their data.

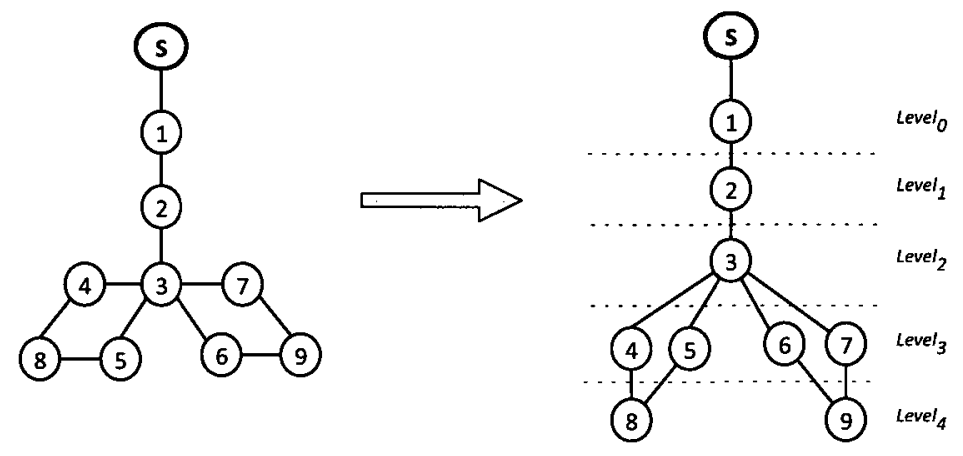

a)

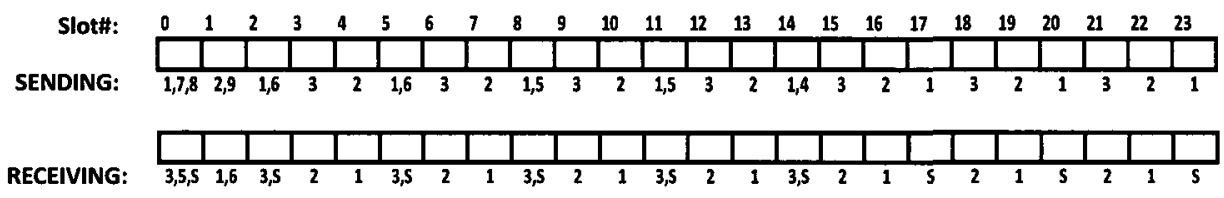

b)
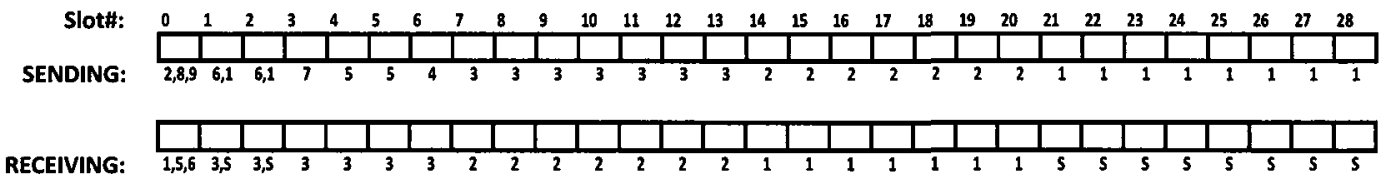

Figure 3.11: An example of schedules generated by the two spatial-temporal interleaved scheduling schemes: (a) Top-down (b) Bottom-Up. Nodes which are neither assigned SEND nor RECEIVE would be assigned SLEEP.

\subsubsection{Leafnode-based Scheduling}

Leafnode-based Scheduling (see A.4.1) performs slot assignments the same way as the Bottom-Up interleaved scheduling, i.e. nodes on the $K-1^{\text {th }}$ level are the first 
ones to be assigned in each cycle followed by nodes at levels closer to the sink, etc. However, leafnode-based scheduling imposes an extra condition regarding nodes that can be assigned SEND in a certain cycle.

- A node $S_{i}$ can be assigned SEND at a specific cycle if and only if $S_{i} \in \mathcal{L}$, where $\mathcal{L}$ is the list of all Leafnodes (a leafnode in Level $_{i}$ is any node whose children (neighbors in Level $_{i+1}$ ) have been assigned SEND in previous cycles).

Figure 3.12 depicts a Leafnode-based schedule for the network used in Figure 3.11. It shows that Leafnode-based scheduling forces nodes in a certain Level $_{i}$ to wait for all of their children in Level $_{i+1}$, to completely transmit their data before they do so.

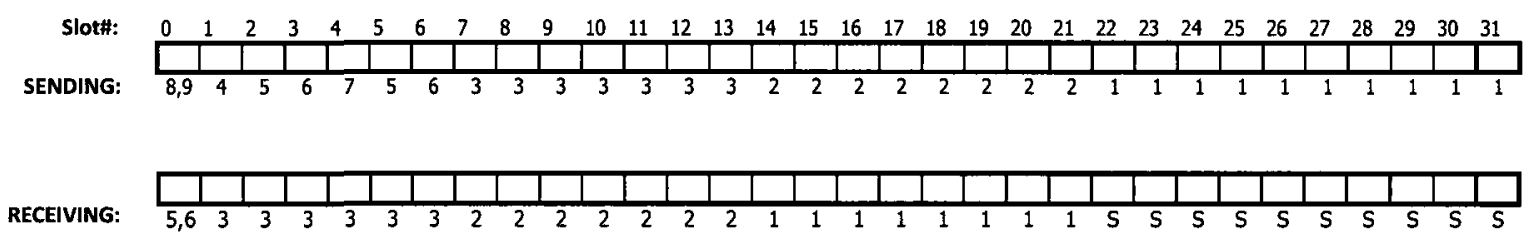

Figure 3.12: Leafnode-based scheduling for the network example used in Figure 3.11.

An important application to the Leafnode-based scheduling is assigning listen windows to nodes during ResP/Sync slots. In Figure 3.13, nodes are assigned multiple sub-slots to receive requests from their children, aggregate the requests and, finally transmit the assembled requests in a single one during a single sub-slot. Another similar application of Leafnode-based scheduling is supporting data aggregation in OCSMACS which is discussed in Chapter 6. 


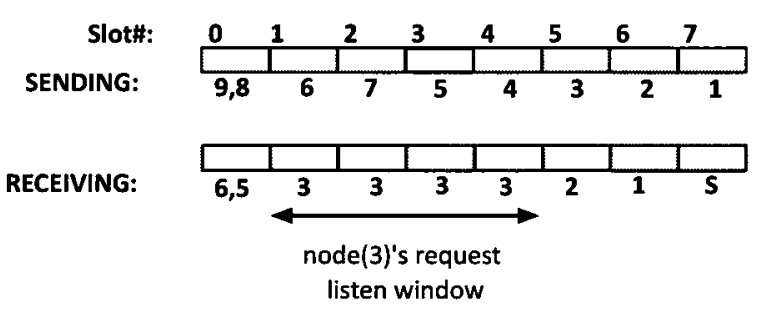

Figure 3.13: Leafnode-based scheduling used to assign listen windows to nodes in Figure 3.11. Node 3 might receive schedule requests during sub-slots $1-4$ and then aggregate them for transmission during sub-slot 5 .

\subsection{Summary}

Existing MAC layer protocols for WSNs are mainly CSMA-based duty cycling protocols. These protocols consume little energy at low traffic conditions. Nevertheless, they lack adaptability to changes in traffic conditions. Moreover, due to the use of CSMA, they poorly handle contention around the sink in convergecast type of applications. On the other hand, scheduling-based (TDMA) protocols are either very rigid in their scheduling schemes or incur high energy cost in the scheduling setup and maintenance phases. Furthermore, most of the existing MAC protocols rely on other routing mechanisms to coordinate the flow of data from sources to destination. The resulting drawback is that routing protocols consume a considerable amount of energy in exchanging routing information.

A MAC protocol called OCSMACS is proposed in this chapter. It addresses the identified issues by using the following mechanism: (a) an adaptive on-demand slot scheduling; (b) compact and aggregate slot assignment requests; and (c) integrated routing/MAC. For OCSMACS's setup phase PROGRESSIVE - a protocol which schedules nodes in a progressive fashion, i.e. nodes are gradually assigned transmission slots, is proposed. Nodes closer to the sink are given higher priority of being scheduled first. This would eventually facilitate scheduling other nodes in the network 
while using a much less signalling overhead. Both PROGRESSIVE and OCSMACS save energy by adopting the discussed mechanisms which substantially minimize idle listening and collisions. 


\section{Chapter 4}

\section{OCSMAC Performance Evaluation}

\subsection{Introduction}

This chapter provides analytical models that characterize the performance of OCSMACS together with the underlying scheduling mechanisms: Top-Down and BottomUp scheduling. Section 4.2 presents a network model, which is used to analyze the proposed scheduling schemes. Expressions of total number of slots and the time required to collect a single data packet from each sensor in the network are given. Section 4.3 presents analytical models to compute the energy consumption for the sensor components (radio and MCU) during different operations, i.e. transmit, receive, listen and sleep.

The presented models in this chapter strongly mirror the behaviour of TD and BU scheduling schemes, the flow of data from sensors to the sink and the operations that affect energy consumption. This chapter does not provide numerical results because to doing so many additional models need to be considered, e.g. the channel model (transmission, recepition, collisions), queueing, generating traffic. These models are 
already part of the simulation and performing numerical analysis of the presented models in this chapter is like a repetition of the simulation.

\subsection{Scheduling Delay}

The objective of this section is to compute the number of slots required to collect data packets from nodes in the network and use the obtained expression to express the delay that a data packet experiences from the time of transmission until it reaches the sink. For this purpose, the network model presented in Section 3.5.1 is used. In addition, the following assumptions are made:

- The network is divided into hypothetical levels based on hop count, so that Level $_{i}$ is $i+1$ hops from the sink. The total number of levels is $k$.

- $N$ is the total number of nodes in the network and there are $n_{i}$ nodes in Level $_{i}$ of the network.

- The time is divided into equivalent time slots. Each time slot $(\tau)$ is long enough for a single packet transmission. In addition, the same slot can be assigned to different nonconflicting nodes (or links).

- $\mathcal{D}_{i}(\tau)$ is the number of data packets in Level $_{i}$ at the beginning of slot $\tau$. Therefore, for each $\operatorname{Level}_{i}, \mathcal{D}_{i}(0)$ is $n_{i}$.

- Let $q_{i}$ denote the proportion of all nodes, which have data in their queues, in Level $_{i}$ transmitting simultaneously without conflicts during slot $\tau$.

- A single data packet is generated from each node in the network. Therefore, the total number of packets to be collected is $N$. 
The objective is to find the required $\tau$ to collect $N$ data packets from $N$ nodes using Top-Down and Bottom-Up scheduling schemes.

\subsubsection{Bottom-Up Scheduling Delay}

Bottom-Up scheduling, as discussed previously, starts the scheduling process by assigning SEND slot to nodes in Level $_{k-1}$. Receiving nodes in Level $_{k-2}$ are assigned RECEIVE. While ensuring that their transmissions would not cause collisions or high interference, all possible non-receiving nodes in Level ${ }_{k-2}$ are assigned SEND. All possible scheduling assignments are performed until Level $_{0}$ is reached. The slot counter is then incremented and the process starts again by assigning SEND to nodes that have not been assigned previously or those having queued packets from previous slots.

For $\tau>0, \mathcal{D}_{i}(\tau)$ is expressed as:

$$
\begin{aligned}
\mathcal{D}_{i}(\tau) & =\mathcal{D}_{i}(\tau-1)+q_{i+1} \mathcal{D}_{i+1}(\tau-1)-q_{i} \mathcal{D}_{i}(\tau-1) \\
& =\left(1-q_{i}\right) \mathcal{D}_{i}(\tau-1)+q_{i+1} \mathcal{D}_{i+1}(\tau-1)
\end{aligned}
$$

For Level $_{L-1}, q_{i+1}$ is zero. Therefore, $\mathcal{D}_{i}(\tau)$ is written as:

$$
\mathcal{D}_{i}(\tau)= \begin{cases}\left(1-q_{i}\right) \mathcal{D}_{i}(\tau-1)+q_{i+1} \mathcal{D}_{i+1}(\tau-1) & 0 \leq i<k-1 \\ \left(1-q_{L-1}\right) \mathcal{D}_{L-1}(\tau-1) & i=k-1\end{cases}
$$

From equation (4.2) the following can be observed:

- Total SEND slots - $\tau_{i}^{S E N D}$ assigned to Level $_{i}$ is $\sum_{\tau=0} q_{i} \mathcal{D}_{i}(\tau)$.

- Total RECEIVE slots - $\tau_{i}^{R E C E I V E}$ assigned to Level $l_{i}$ is $\sum_{\tau=0} q_{i+1} \mathcal{D}_{i+1}(\tau)$. 
- Total SLEEP slots $-\tau_{i}^{S L E E P}$ assigned to Level $_{i}$ is $\tau_{i}^{\text {total }}-\tau_{i}^{\text {SEND }}-\tau_{i}^{R E C E I V E}$. $\tau_{i}^{\text {total }}$ is the total number of slots assigned to nodes in Level $_{i}$.

- The time required to collect packets from all nodes $T_{m u l t i h o p}^{B U}$ is:

$$
T_{\text {multihop }}^{B U}=\min (\tau) \times T_{\text {data-slot }}^{\text {OCSMACS }} \quad \text { s.t. } \quad \sum_{i=0}^{L-1} \mathcal{D}_{i}(\tau)=0
$$

It is evident from Equations (4.2) and (4.3) that to minimize $T_{\text {multihop }}^{B U}, \mathcal{D}_{i}(\tau)$ needs to be minimized for all $i$. One way to achieve this is by maximizing the number of packets transmitted during slot $\tau$. In other words, $q_{i} \mathcal{D}_{i}(\tau)$ is maximized. The limiting factor is $q_{i}$ and to maximize $q_{i} \mathcal{D}_{i}(\tau), q_{i}$ needs to be maximized during each slot.

\subsubsection{Top-Down Scheduling Delay}

Unlike Bottom-Up scheduling, Top-Down scheduling always begins by assigning a Send slot to a node in Level $_{0}$ in slot $(\tau=0)$, then assigning SEND to all possible nodes in Level $_{1}$ as long as collisions or high interference are prevented. Receiving nodes in Level $_{0}$ are assigned REceive. The scheduling process is repeated for the remaining levels until further slot assignment is not possible in the same slot $\tau$. Afterwards the slot counter is incremented and the whole process is repeated starting from Level $l_{0}$ until all data packets are collected from nodes. 
In Top-Down scheduling, $q_{0}$ is at most $1 / n_{0}$. Hence, $\mathcal{D}_{i}(\tau)$ is:

$$
\mathcal{D}_{i}(\tau)= \begin{cases}\left(1-\frac{1}{n_{0, \tau}}\right) \mathcal{D}_{0}(\tau-1)+q_{1} \mathcal{D}_{1}(\tau-1) & i=0 \\ \left(1-q_{i}\right) \mathcal{D}_{i}(\tau-1)+q_{i+1} \mathcal{D}_{i+1}(\tau-1) & 0<i<k-1 \\ \left(1-q_{L-1}\right) \mathcal{D}_{L-1}(\tau-1) & i=k-1\end{cases}
$$

The time $T_{\text {multihop }}^{T D}$ required to collect packets from all nodes is:

$$
T_{\text {multihop }}^{T D}=\min (\tau) \times T_{\text {slot }}^{O C S M A C S} \quad \text { s.t. } \quad \sum_{i=0}^{L-1} \mathcal{D}_{i}(\tau)=0
$$

Top-Down scheduling guarantees that a single packet is transmitted from a node in Level $_{0}$ in each time slot. This maintains a constant reduction in $\mathcal{D}_{0}(\tau)$ by a factor of $1 / n_{0}$ (equivalent to one packet). In addition, in Bottom-Up scheduling, the rate at which $\mathcal{D}_{i}(\tau)$ decreases as $i \rightarrow k-1$ is lower than that of Top-Down scheduling. For these reasons Top-Down scheduling is more likely to have smaller $T_{m u l t i h o p}^{T D}$ than $T_{m u l t i h o p}^{B U}$ as previously demonstrated in the example of Figure 3.11.

\subsection{Energy Consumption}

In order to model the energy consumption of OCSMACS, energy consumption of the individual components of individual sensor nodes, which contribute to the overall energy consumption is computed. Mainly, the radio communication as well as the micro-controller (MCU) are considered. A network model where $N$ nodes are uniformly distributed in a circular field and density of $\delta_{s n}$ (nodes $\left./ m^{2}\right)$ is used. Each node is assumed to transmit at a maximum transmission range $\mathcal{R}_{s n}$ while the sink, 
located in the centre, transmits at a maximum range $\mathcal{R}_{\text {sink }}$ so that $\mathcal{R}_{\text {sink }}=k \times r_{n}^{\prime}$ $\left(r_{s n}^{\prime}=\mathcal{R}_{s n}-\Delta \mathcal{R}_{s n}\right)$ is also the field radius.

The whole network area is divided into $k$ hypothetical concentric rings each of depth $d=r_{s n}^{\prime}$ centred at the $\operatorname{sink}^{1} . \Delta \mathcal{R}_{s n}$ is necessary to ensure that each node in ring $_{i}$ can communicate with at least one node in ring $_{i-1}$, i.e. all nodes which are $i$ hops from the sink are in $r_{i n g}$. As Figure 4.1 shows, ring $_{0}$ is the circle with radius $r_{s n}$ and $\operatorname{ring}_{1}$ is the shaded ring.

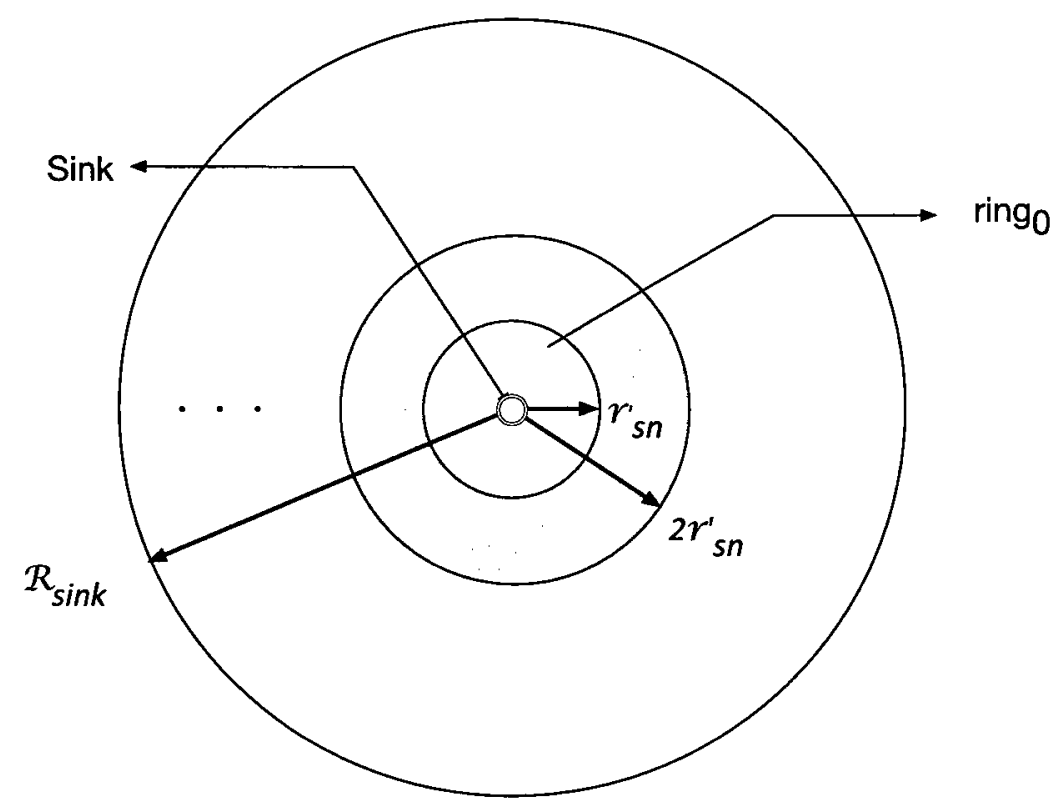

Figure 4.1: Multiple rings network representation.

Based on this model, the following assumptions and definitions are given:

1. All nodes draw the same current (or power) levels when communicating data (all nodes use the same maximum $\mathcal{P}_{s n}$ ) or when carrying out internal processing tasks.

\footnotetext{
${ }^{1}$ Network analysis based on similar models can be found in [59], [95] and [96], for example.
} 
2. All nodes have the same sampling rate $r_{\text {sampling }}$ and the same packet length $P K T_{\text {length }}$ (in bytes).

3. In a convergecast application, packets transmitted by nodes in ring $_{i}$ must be relayed by those in $\operatorname{ring}_{i-1}$, where $0<i \leq k-1$.

4. Let $\lambda_{i}$ be the total number of packets generated (sampled) by nodes in ring . $_{\text {. }}$

5. Let $\lambda_{i}^{t x}$ denote the total number of packets transmitted by nodes in ring $g_{i}$ and received by nodes in ring $_{i-1}$.

6. Let $\lambda_{i}^{r x}$ denote the total number of packets received by nodes in $r i n g_{i}$.

\subsubsection{Radio Energy}

In this section, a set of expressions for the radio energy consumption during transmission, receiving, idle listening, switching and sleeping are presented.

\section{Transmission Energy}

The transmission energy component refers to the energy consumed for transmitting packets over the radio. It can be expressed as:

$$
E^{t x}=\lambda^{t x} \times P K T_{\text {length }} \times T_{\text {Byte }} \times \mathcal{P}_{t x}
$$

where $\lambda^{t x}$ is the total number of data packets transmitted, $T_{B y t e}$ is the time for transmitting one byte over the radio and $\mathcal{P}_{t x}$ is the power consumption of the radio while in transmit mode.

The total number of data packets transmitted $\lambda^{t x}$ depends on two variables: 
1. The sampling rate, $r_{\text {sampling }}$.

2. The relayed traffic; nodes in $r i n g_{i}$ relay packets transmitted by nodes in ring $_{i+1}$. Therefore, each $\operatorname{ring}_{i}$ might have a different $\lambda^{t x}$. The total number of packets $\lambda_{i}^{t x}$ transmitted by ring $_{i}$ is written as:

$$
\lambda_{i}^{t x}=\lambda_{i}+\lambda_{i+1}^{t x}
$$

The total transmission energy $E_{t x}$ is expressed as the sum of transmission energies of all rings:

$$
\begin{aligned}
E^{t x} & =\sum_{i=0}^{k-1} E_{i}^{t x} \\
& =\sum_{i=0}^{k-1} \lambda_{i}^{t x} \times P K T_{\text {length }} \times T_{\text {Byte }} \times \mathcal{P}_{t x}
\end{aligned}
$$

Few expressions are developed to compute $\lambda_{i}^{t x}$ and $E_{i}^{t x}$ of each ring. Let $A_{i}$ denotes the area of $r i n g_{i}$. Then $A_{i}=\pi\left((i+1) r_{n}^{\prime}\right)^{2}-\pi\left(i r_{n}^{\prime}\right)^{2}$. Let $n_{i}$ be the number of nodes in ring $_{i}$. Since nodes are distributed uniformly with density $\delta_{s n}$, then $n_{i}$ is $\delta_{s n} A_{i}$. During a period of time $t, \lambda_{i}^{t x}$ is:

$$
\begin{gathered}
\lambda_{i}^{t x}=t \times r_{\text {sampling }} \times n_{i}+\lambda_{i+1}^{t x} \\
=t \times r_{\text {sampling }} \times \delta_{s n} A_{i}+\lambda_{i+1}^{t x} \\
\lambda_{i}^{t x}= \begin{cases}t \times r_{\text {sampling }} \times \delta_{s n} A_{0}+\lambda_{1}^{t x} & i=0 \\
t \times r_{\text {sampling }} \times \delta_{s n} A_{i}+\lambda_{i+1}^{t x} & 0<i<k-1 \\
t \times r_{\text {sampling }} \times \delta_{s n} A_{k-1} & i=k-1\end{cases}
\end{gathered}
$$




\section{Receiving Energy}

The reception energy component refers to the energy consumed while receiving packets on the radio. It is expressed as:

$$
E^{r x}=\lambda^{r x} \times P K T_{\text {length }} \times T_{\text {Byte }} \times \mathcal{P}_{r x},
$$

where $\mathcal{P}_{r x}$ is the power consumption of the radio while in receive mode.

Similar to transmission energy, the reception energy $E^{r x}$ is the sum of reception energies of all rings:

$$
\begin{aligned}
E^{r x} & =\sum_{i=0}^{k-1} E_{i}^{r x} \\
& =\sum_{i=0}^{k-1} \lambda_{i}^{r x} \times P K T_{\text {length }} \times T_{\text {Byte }} \times \mathcal{P}_{r x}
\end{aligned}
$$

During a period of time $t, \lambda_{i}^{r x}$ is given by:

$$
\begin{aligned}
\lambda_{i}^{r x} & =\lambda_{i+1}^{t x} \\
& =t \times r_{\text {sampling }} \times \delta_{s n} A_{i+1}+\lambda_{i+1}^{r x} \quad 0 \leq i<k-1
\end{aligned}
$$

\section{Listening Energy}

Listening, or idle listening, is the energy consumed when the radio remains in the active mode without receiving or transmitting any packets. In OCSMACS, idle listening happens in two cases:

1. When nodes wake-up during RESP/SynC (request or synchronization slot) to receive possible requests from their neighbors but most or all receive no requests. 
Therefore, nodes are in an idle listening state for the whole listen window.

2. When the schedule request aggregation algorithm redundantly sets some nodes as forwarders (relays) of traffic but no forwarding is required. These nodes consume energy while sampling the channel at the beginning of each RECEIVE slot assigned to them expecting them to relay traffic.

In a typical network-wide data gathering application, each node transmits a single schedule request. Eventually, all requests are aggregated and the next ScHDL frame would include a slot assignment for all nodes. However, due to transmission errors and/or request elimination during schedule aggregation, some nodes might need to retransmit their requests during upcoming SCHEduleREQ slots.

During a period of time $t$, the number of schedule requests transmitted $N_{R E Q}^{t x}(t)$ is expressed as:

$$
N_{R E Q}^{t x}(t)=1+\varepsilon
$$

where $\varepsilon$ accounts for any schedule request retransmissions.

However, when the number of sources and sampling rate varies considerably overtime, then $N_{R E Q}^{t x}(t)$ will depend on two factors:

1. The sampling rate $r_{\text {sampling }}$.

2. The active source timer $t_{\text {active-source }}(n)$. It is assumed that the sink sets the duration for the timer to be of the same length as the current frame, i.e. $t_{\text {active-source }}(n)=T_{\text {frame }}$ 
Based on these factors, $N_{R E Q}^{t x}(t)$ is:

$$
N_{R E Q}^{t x}(t)= \begin{cases}1+\epsilon & \frac{1}{r_{\text {sampling }}} \leq T_{R E Q-S C H D} \\ t \times r_{\text {sampling }}+\epsilon & \frac{1}{r_{\text {sampling }}}>T_{R E Q-S C H D}\end{cases}
$$

where $T_{R E Q-S C H D}$ is expressed as :

$$
2 \times T_{\text {frame }}
$$

Given $N_{R E Q}^{t x}(t)$, the number of ScheduleREQ slots, $N_{R E Q}^{i d l e}(t)$, during which nodes will be idle listening is given by:

$$
N_{R E Q}^{i d l e}(t)=\left\lceil\frac{t}{T_{\text {frame }}}\right\rceil-N_{R E Q}^{t x}(t)
$$

Another scenario that brings about an idle listening state is when a node is assigned a RECEIVE slot but nothing is received. In this case, the listening time is:

$$
T_{\text {listen }}^{L P L}=\tau_{\text {unused }}^{R E C I V E} \times T_{\text {sample }}
$$

where $\tau_{\text {unused }}^{\text {RECIVE }}$ is the total number of unused RECEIVE slots and $T_{\text {sample }}$ is the time spent sampling the channel at the beginning of a RECEIVE slot. The total idle listening energy consumption is:

$$
E_{\text {listen }}=\left[N_{R E Q}^{i d l e}(t) \times T_{R L W}+T_{l i s t e n}^{L P L}\right] \times \mathcal{P}_{l i s t e n}
$$


where $T_{R L W}$ is the request listen window duration and $\mathcal{P}_{\text {listen }}$ is the power consumption of the radio while idle listening.

\section{Switching Energy}

The switching, or radio setup, energy component is the energy consumed for switching the radio state between sleep and active modes. In OCSMACS, switching might happen when nodes wake-up to check for schedule requests. In addition, nodes may wake-up from the sleep mode to receive synchronization messages or SCHEDULE frames. Furthermore, switching happens between SLEEP and REcEIVE/SEND slots.

The switching energy can be expressed as:

$$
E_{\text {switch }}=\left[S W_{R E Q}+S W_{R E S P / S Y N C}+S W_{\text {scheduling }}\right] \times T_{\text {switch }} \times \mathcal{P}_{\text {switch }},
$$

where $S W_{R E Q}$ and $S W_{R E S P / S Y N C}$ denote number of times a node switches ON its radio to receive schedule requests from neighboring nodes and to receive ScHDL frames or synchronization, respectively. $S W_{\text {scheduling }}$ is the number of times a node switches its radio between SLEEP and RECEIVE/SEND slots during data exchange periods (active frames), $T_{\text {switch }}$ is the switching time and $\mathcal{P}_{\text {switch }}$ is the radio power consumption when turned ON from sleep mode.

\section{Sleep Energy}

The radio switches to the sleep mode when no communication activity is taking place. Therefore, sleep time $T_{r f}^{\text {sleep }}$ is:

$$
T_{r f}^{\text {sleep }}=t-T_{T X}-T_{R X}-T_{L I S T E N}-T_{S W I T C H}
$$


where, $T_{T X}, T_{R X}, T_{L I S T E N}, T_{S W I T C H}$ is the total time that the radio spends transmitting, receiving, listening and switching, respectively. The energy consumed during sleep mode is:

$$
E_{\text {sleep }}=T_{r f}^{\text {sleep }} \times \mathcal{P}_{r f}^{\text {sleep }}
$$

where $\mathcal{P}_{r f}^{\text {sleep }}$ is the radio power consumption during sleep mode.

\subsubsection{Micro-controller Energy}

The MCU operates in one of two modes at any one time: active or sleep (power down). It remains active when communication is taking place, or when carrying other tasks, e.g. signal processing. However, in this part of the analysis, the MCU is considered active only during data communications. Chapter 6 , however, discusses the energy consumption during data aggregation.

The MCU active time $T_{M C U}^{a c t i v e}$ is expressed as:

$$
T_{M C U}^{a c t i v e}=T_{T X}+T_{R X}+T_{L I S T E N}
$$

The MCU active energy is:

$$
E_{M C U}^{a c t i v e}=T_{M C U}^{a c t i v e} \times \mathcal{P}_{M C U}^{a c t i v e}
$$

where $\mathcal{P}_{\mu C}^{a c t i v e}$ is the MCU power consumption during active operation.

During sleep mode the MCU energy consumption is:

$$
E_{M C U}^{s l e e p}=T_{M C U}^{s l e e p} \times \mathcal{P}_{M C U}^{s l e e p}
$$


where $T_{M C U}^{\text {sleep }}=t-T_{M C U}^{a c t i v e}$ is the total time during which the MCU is in sleep mode and $\mathcal{P}_{M C U}^{\text {sleep }}$ is the power consumption of the MCU while the node is not active. The total MCU energy consumption is then:

$$
E_{M C U}=E_{M C U}^{a c t i v e}+E_{M C U}^{\text {sleep }}
$$

\subsubsection{Optimizing Network Lifetime}

As discussed in Chapter 1, extending network lifetime is a primary objective in most WSN applications. The definition of network lifetime depends on the specific application. One possible definition is the time until the first node fails (or runs out of energy). Other definitions include the time until the network is disconnected into different partitions or the time until some percentage $(x \%)$ of nodes fail.

For convergecast applications, nodes that are close (one or two hops) to the sink consume a significant amount of energy compared to other nodes. This occurs because these nodes are the primary gateways through which data is forwarded to the sink. If these nodes run out of energy, then data collected by other nodes in the network cannot be communicated to the sink. Therefore, network lifetime for convergecast applications may be defined as the time until the first node within a single hop from the sink dies. To formally express this definition, let $T_{s n_{i}^{k}}$ denote the lifetime of node $s n_{i}$ at the $k^{t h}$ hop from the sink. Then the network lifetime is $T_{n e t}=\min _{i=1} T_{s n_{i}^{k}}$.

\section{Notations}

The following notations are used:

- Let $E^{h(k)}$ denote the total energy consumed by a node in the $k^{t h}$ hop. 
- $\lambda_{s n_{i}}$ is the total number of data packets generated by a node $s n_{i}, i \in 0, \ldots, N$.

- $\lambda_{\text {sink }}^{s n_{i}}$ is the total number data packets collected at the sink and originated from node $s n_{i}$. The MAC protocol should have the mechanisms to ensure that:

$$
\lambda_{\text {sink }}=\sum_{i=0}^{N-1} \lambda_{\text {sink }}^{s n_{i}}=\sum_{i=0}^{N-1} \lambda_{s n_{i}}=\sum_{i=0}^{N-1}\left(T_{n e t} \times r_{\text {sampling }}\right) .
$$

- Let $Q_{s n_{i}(t)}$ be the queue size of node $s n_{i}$ at time $t$.

\section{WSN Lifetime}

The optimization problem to maximize the lifetime $T_{\text {net }}$ of a WSN running a convergecast application can be formulated based on the following constraints:

1. Energy constraint: the total energy consumed by each node in the $1^{\text {st }}$ hop $\left(E^{h(1)}\right)$ during time $T_{n e t}$ should be less than or equal to $E_{s n}$, the initial energy of each node.

2. Data delivery constraint: the MAC protocol should maximize data delivery, i.e. maximize $\lambda_{\sin k}$.

Therefore, network lifetime maximization is:

$$
\begin{array}{ll}
\max . & T_{\text {net }} \\
\text { s.t. } & E^{h(1)} \leq E_{s n} \\
& E^{h(1)}=E_{t x}^{h(1)}+E_{r x}^{h(1)}+E_{\text {listen }}^{h(1)}+E_{\text {switch }}^{h(1)}+E_{\text {sleep }}^{h(1)}+E_{\mu C}^{h(1)}
\end{array}
$$

OCSMACS works in an adaptive fashion so that slots are assigned to nodes on demand. The computed schedule contributes in maximizing $T_{n e t}$ by assigning slots 
only to active nodes. In addition, it is assumed that separation between nodes is in the range of a few tens of meters and all nodes transmit at a power level high enough to have a range that is as long as the maximum separation distance. Therefore, $T_{\text {net }}$ maximization, as presented in this work, does not take into account assigning variable transmission powers to nodes. Instead, maximizing the sleep time of nodes is considered the most effective solution to maximize $T_{n e t}$. However, there are some factors that negatively affect this approach:

1. Delay: increasing the sleep time of nodes means that data will be queued for a long period before being transmitted. Hence, the end-to-end delay increases.

2. Queue size: sensor nodes have very constrained memory sources. Long sleep periods will force data to be queued for later transmissions. As both the sampling rate and sleep periods increase, queue size increases and data becomes subject to loss, i.e. $\lambda_{\text {Sink }}<\sum_{i=0}^{N-1} \lambda_{s n_{i}}$.

Accordingly, maximizing lifetime of a WSN running OCSMACS is defined as a problem to maximize $T_{\text {sleep }}$. Maximizing OCSMACS's sleep time is possible by maximizing $\mathcal{S}$, the number of data exchange slots in the frame. The realtion between $\mathcal{S}$ and $T_{n e t}$ is that if $\mathcal{S}$ is much larger than the actual number of required slots to collect data from all nodes in the network, then there would larger number of SLEEP slots in the frame. Due to the delay and queue size factors, two additional constraints must be satisfied while using this approach:

1. Frame constraint: the number of data exchange slots $(\mathcal{S})$ must satisfy:

$$
\mathcal{S} \leq\left\lfloor\frac{\text { delay }_{\max }-\left[T_{R E Q}+T_{\text {scheduling }}^{\text {Sink }}+T_{S C H D}+T_{\text {multihop }}\right]}{\left.T_{\text {data }}^{\text {OCSMACS }}\right\rfloor}\right.
$$


2. Queue size constraint: total data queued at each node must remain below a threshold to prevent queue overflow and delays beyond the acceptable levels for the application.

Therefore, network lifetime maximization is:

$$
\begin{array}{ll}
\max . & \mathcal{S} \\
\text { s.t. } & E^{h(1)} \leq E_{\text {sn }} \\
& \text { delay }_{\max }>\left[T_{R E Q}+T_{\text {scheduling }}^{\text {Sink }}+T_{S C H D}+T_{\text {multihop }}\right] \\
& Q_{\text {sni }(t)}<Q^{\text {max }}, \forall s n_{i}, i \in\{0, \ldots, N\}, t \leq T_{\text {net }} \\
& \lambda_{\text {Sink }} \text { is maximized }
\end{array}
$$

The variables are $r_{\text {sampling }}$ and $N$ (or $\left.\delta_{s n}\right) . r_{s a m p l i n g}$ affects $\lambda_{s n_{i}}$ and subsequently affects $E_{s n}$ while $N$ affects $T_{R E Q}, T_{S C H D}, T_{\text {multihop }}$ and $T_{\text {scheduling }}^{\text {sink }}$. Therefore, the optimal $\mathcal{S}$ that maximizes network lifetime, for a given sampling rate and/or network size or density, is computed.

\subsection{Summary}

In this chapter, mathmatical expressions that model delay and energy consumption of OCSMACS have been presented. Specifically, expressions for TD and BU multihop scheduling delay are given. It was shown that $\mathrm{BU}$ scheduling tends to have more delay than TD scheduling. The resaon is that in TD gives higher priority in assign transmission slots to nodes that are close to the sink. This reduces congestion from these nodes hence resulting in less number of slots, compared to BU. In terms of energy consumption, models for radio energy consumption are given. This includes 
transmission, reception, idle listening, switching and sleep energy. In addition, MCU active and sleep energy consumption is considered.

Maximizing network lifetime is shown to be a problem of maximizing OCSMACS frame size (maximizing the number of data slots, $\mathcal{S}$ ). This approach is is used because as $\mathcal{S}$ becomes much larger than the actual number of required slots to collect data from all nodes in the network, then there would larger number of SLeEP slots in the frame. Therefore, nodes would sleep more and conserve energy. It was observed that several factors would affect this approach and need to be considered in finding the optimal value of $\mathcal{S}$. Examples of these factors include: maximum end-to-end delay set by the application and maximum queue size of each node. 


\section{Chapter 5}

\section{Simulation Evaluation}

\subsection{Introduction}

This chapter presents an ns-2 [9] simulation-based evaluation for PROGRESSIVE and OCSMACS. Simulations are divided into two parts:

- Part I Evaluates and compares PROGRESSIVE, the proposed progressive topology learning and scheduling protocol, against DRAND ${ }^{1}[10]$.

- PartII Evaluates and compares the performance of OCSMACS to that of the following protocols:

1. ALWAYS-ON: A CSMA protocol similar to the 802.11 protocol without power saving and without collision avoidance (RTS/CTS).

2. S-MAC: A full S-MAC ${ }^{2}$ implementation as proposed in [11] with adaptive listening enabled. The active period is set to accommodate one packet

\footnotetext{
${ }^{1}$ Implementation is available by one of the authors at http://www4.ncsu.edu/rinee/export/zmac/ software/drand/drand.htm.

${ }^{2}$ Implementation of S-MAC in $n s-2$ distribution 2.31.
} 
transmission plus ACK. Packet size is assumed to be small such that RTS/CTS and message passing options are not activated.

3. DMAC: This is the $\mathrm{DMAC}^{3}[12]$ protocol with and without the More-ToSend (MTS) option. The active and the sleep periods as well as the data collection tree are assumed to be predetermined.

4. Z-MAC: The Z-MAC ${ }^{4}[13]$ protocol where the data collection tree is assumed to be predetermined. In addition. the transmission schedules are recomputed using DRAND.

\subsection{Assumptions and Default Parameters}

Simulations results presented in this chapter are averages of 20 independent (different seed) replications such that each replication has a different topology, data generation start times, etc. In addition, all results are plotted with $95 \%$ confidence intervals as described in Appendix B. For OCSMACS, Z-MAC, S-MAC, DMAC and ALWAYSON, it is assume that the routing paths are already predetermined. This would eliminate initial delays caused during acquiring these routing paths. In addition, data generation stops before terminating the simulation for a time duration long enough to collect statistics of all packets in transit. This time is two to three times the worst end-to-end delay.

Table 5.2 shows time and energy comparison of MICA2 [99, 100, 68] sensors assumed in the simulations. The choice of MICA2 [23] sensor platform for evaluating

\footnotetext{
${ }^{3}$ Implementation is available by one of the authors at http://anrg.usc.edu/www/index.php /Downloads.

${ }^{4}$ Implementation is available by one of the authors at http://www4.ncsu.edu/rinhee/export/zmac/ software/zmac/zmac.htm.
} 
OCSMACS is that it would make it possible to validate and compare our results with those of other protocols. With respect to the sink, it is assumed to have an unlimited source of energy.

\begin{tabular}{|l|l||l|l|}
\hline Nodes' Placement & Randomly uniform & Avg. Node Degree & 5 \\
\hline Sink Placement & Center of the field & Number of Sink(s) & One \\
\hline Field Geometry & Two-dimensional & Field Size & Varies \\
\hline Transmission Range & $20 m$ & Interference Range & $45 m$ \\
\hline Sensor Platform & MICA2 & Data Rate & 38.4 Kbps \\
\hline Data Payload & 29 bytes & ACK & 14 bytes \\
\hline
\end{tabular}

Table 5.1: Default Simulation Parameters.

\begin{tabular}{|l|l||l|l|}
\hline Turn on radio & $1.5 \mathrm{~ms}$ & Switch to RX/TX & $250 \mu \mathrm{s}$ \\
\hline Time to sample radio & $350 \mu \mathrm{s}$ & Evaluate radio sample & $100 \mu \mathrm{s}$ \\
\hline \hline Radio Tx Current & $16.5 \mathrm{~mA}$ & Radio Rx Current & $9.6 \mathrm{~mA}$ \\
\hline Radio Idle Current & $7.68 \mathrm{~mA}$ & Radio Sleep Current & $0.2 \mu \mathrm{A}$ \\
\hline MCU Active Current & $8 \mathrm{~mA}$ & MCU Sleep Current & $14 \mu \mathrm{A}$ \\
\hline
\end{tabular}

Table 5.2: MICA2 Sensor Timings and Power Consumption.

\subsection{PROGRESSIVE Performance}

In evaluating the performance of PROGRESSIVE, the following set of criteria is considered:

1. Energy Efficiency: The total energy consumed during the topology discovery and the scheduling phases.

2. Scheduling Efficiency: The percentage of nodes that have been successfully assigned transmission slots without any conflict. 
3. Time: The total time required to achieve the maximum possible scheduling efficiency.

4. Neighborhood Knowledge: The percentage of each node's neighbors that have been identified at the end of the neighbor discovery phase. For DRAND, the neighborhood knowledge is the average neighborhood knowledge of all nodes. However, for PROGRESSIVE, neighborhood knowledge is computed at the sink based on the information that reaches the sink. Since the sink might not receive NET-LIST from each individual node, it might not have a complete knowledge about all neighbors of each individual node. The lack of knowledge about some nodes' neighbors would lower neighborhood knowledge and might lead to conflicts in slot assignments.

5. Number of Message transmissions: The total number of control messages transmitted by each node during the topology discovery and the scheduling phases.

Various simulations are carried out to study the effects of the neighbor discovery duration $t_{\text {discovery }}$, the number of nodes $N$ and the relative node density. Results are the average of 20 simulations. Each simulation uses a different random seed and runs for 15 minutes. This simulation time is chosen because in the case of DRAND it is long enough to schedule the minimum number of nodes, i.e. 40 nodes, as well shown later. It is long enough to demonstrate the difference in scheduling times of both protocols. In addition, all results are plotted with $95 \%$ confidence intervals. Table 5.1 shows the default parameters used. For the energy comparison, it is assumed that the sink has an unlimited source of energy. DRAND-specific parameters are set based 
on the values in [10]. However, the maximum number of slots is set large enough so that typically DRAND is able to schedule all nodes. DRAND results presented in this section differ from those in [10] mainly because in [10] a data rate of $2 \mathrm{Mbps}$ is used. However, our simulations assume a MICA2 platform with a $38.4 \mathrm{Kbps}$ data rate. Nonetheless, performance of both protocols with a $2 \mathrm{Mbps}$ data rate is discussed in section 5.3.5.

\subsubsection{Net-List Backoff Window Size}

The size of the NET-LIST backoff window $B W_{N L I S T}$ is important in reducing contention among nodes across different hops during the NET-LisT broadcast period. Our objective is to maximize the number of NET-LIST packets that reach the sink by the end of the topology learning phase so that the sink has more information about the topology. This increases the number of nodes that can be scheduled. The size of $B W_{N L I S T}$ has a direct effect on the number of NET-LisT packets that can reach the sink.

To study this effect, 100 nodes are simulated varying $R$ between $[4,8,16,32,64]$. Figure 5.1 shows the percentage of each hop's nodes that are assigned transmission slots for different values of $R$. When $R$ is 64 , almost $100 \%$ of nodes at hop counts 1, 2 and 3 are scheduled, while few nodes in the large hop counts are scheduled. The reason for this is that when $R$ becomes large, nodes which are close to the sink have a better chance of broadcasting NET-LIST packets before the end of the topology learning phase compared to nodes at higher hop counts. When $R$ is 4 , however, nodes at large hop counts have a better chance of being assigned transmission slots than is the case when $R$ is 64 . It can be observed that a balance in the number of nodes 
scheduled from each hop is achieved when $R$ is 16 . Figure 5.2 demonstrates that the maximum number of nodes which are scheduled on the first frame occurs when $R$ is 16. The reason is that the number of nodes at higher hop counts is larger than that in the first or the second hop count. By increasing the chance of nodes to transmit their NET-Lists, the number of scheduled nodes in the first frame increases.

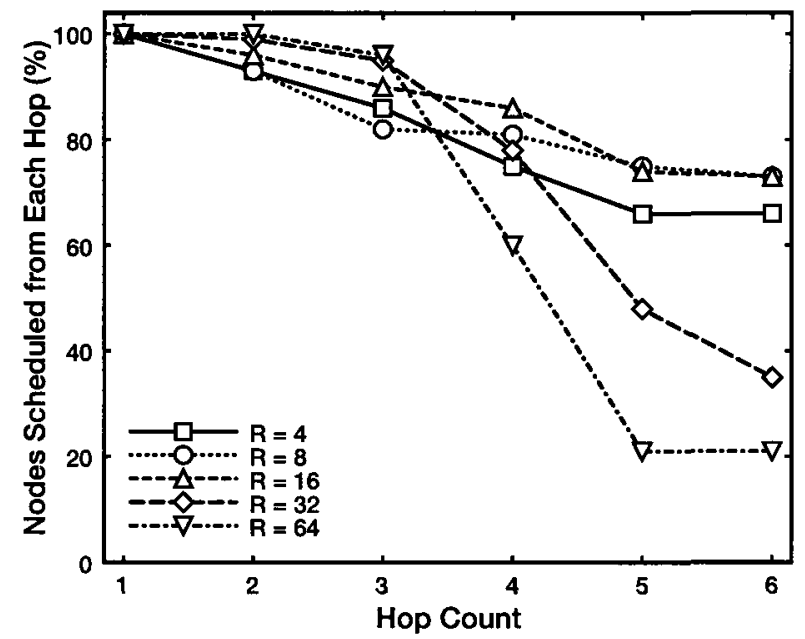

Figure 5.1: Percentage of nodes which are scheduled from each hop as $R$ is varied, $N=$ 100 .

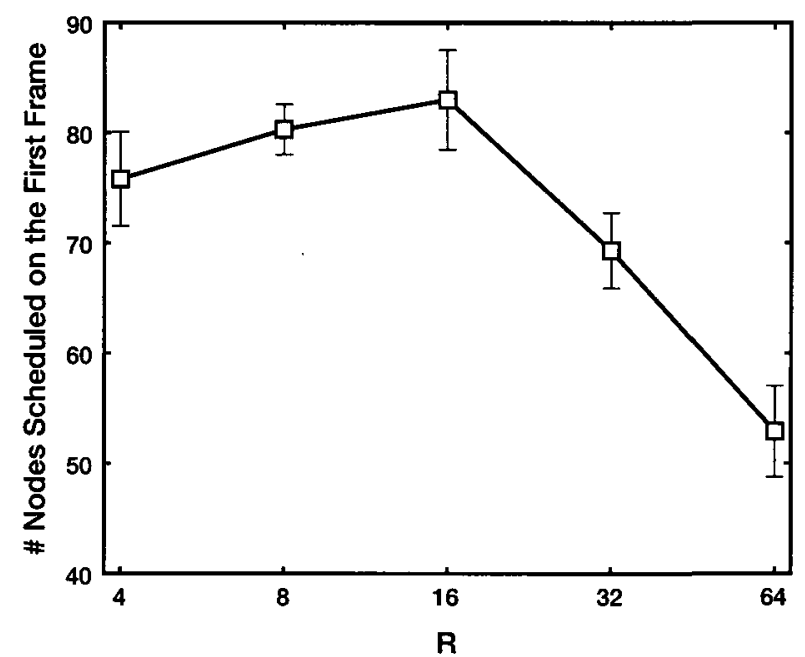

Figure 5.2: Total number of nodes which are scheduled as $\mathrm{R}$ is varied, $N=100$. 


\subsubsection{Neighbor Discovery Duration}

In studying the impact of the neighbor discovery duration $t_{\text {discovery }}$, a network of 80 nodes is simulated varying $t_{\text {discovery }}$ among $[2,4,8,16,32,64,128] \mathrm{s}$. With respect to PROGRESSIVE, $t_{\text {discovery }}$ has a most notable effect on the neighborhood knowledge (see Figure 5.3(a)). A short $t_{\text {discovery }}$ means few Hello messages are exchanged among neighboring nodes and fewer NET-Lists reach the sink. Consequently, the sink has little knowledge about the network topology. Increasing $t_{\text {discovery }}$ provides more time for nodes to exchange Hello messages and to broadcast NeT-Lists hence, the sink has more information about the topology.

Figure 5.4(a) shows that when $t_{\text {discovery }}$ is small, e.g. 2 s, about 311 Hello and 388 NET-LIST messages are transmitted. However, this causes an increase in the number of ScHDL frames (15 frames). This occurs because of insufficient neighborhood knowledge at the sink results in an initial schedule where a large number of nodes are not scheduled or have conflicts. Each unscheduled node independently notifies the sink during the adjustment phase. When the sink receives a notification (NET-LIST), a new slot assignment is computed and a ScHDL frame transmitted. Increasing $t_{d i s c o v e r y}$ to $128 \mathrm{~s}$ increases message transmissions (138 HeLlo and 28 NeT-LIST) and allows the sink to perform slot assignment based on better knowledge of the topology. Therefore, fewer ScHDL frames are transmitted, 2 frames when $t_{\text {discovery }}$ is $128 \mathrm{~s}$. Despite the variations in $t_{\text {discovery }}$ and the number of message transmissions, PROGRESSIVE is able to achieve $100 \%$ scheduling efficiency (Figure 5.3(c)) because of its novel mechanism, which gradually schedules nodes even with little initial topology information.

With respect to DRAND, increasing $t_{\text {discovery }}$ over $4 \mathrm{~s}$ does not affect the neighborhood knowledge because in 4s each node is able to obtain full knowledge of its 


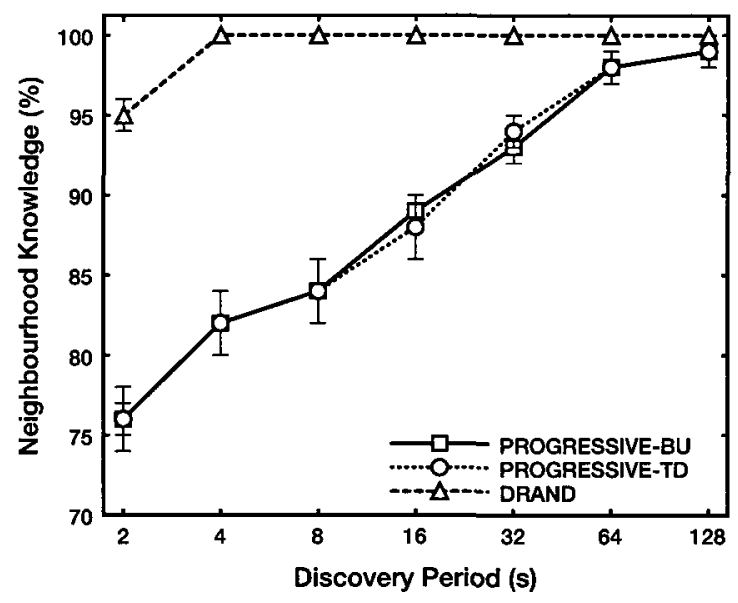

(a) Neighborhood Accuracy

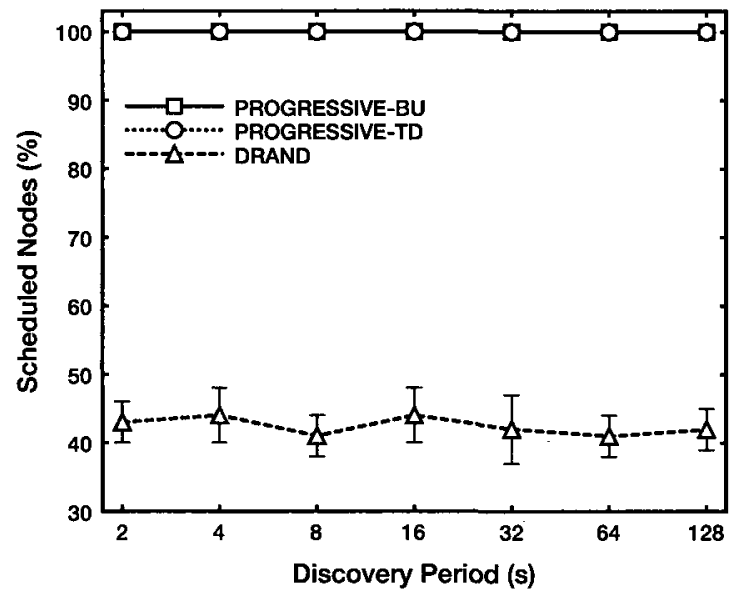

(c) Scheduling Efficiency

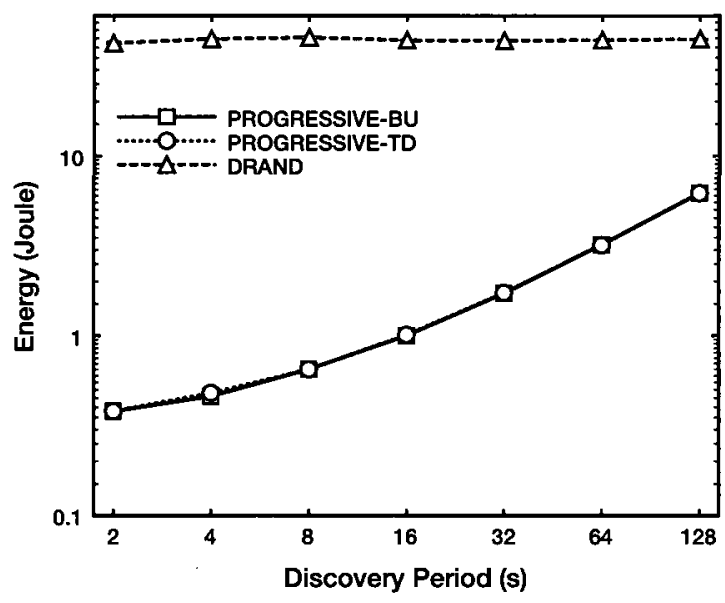

(b) Energy

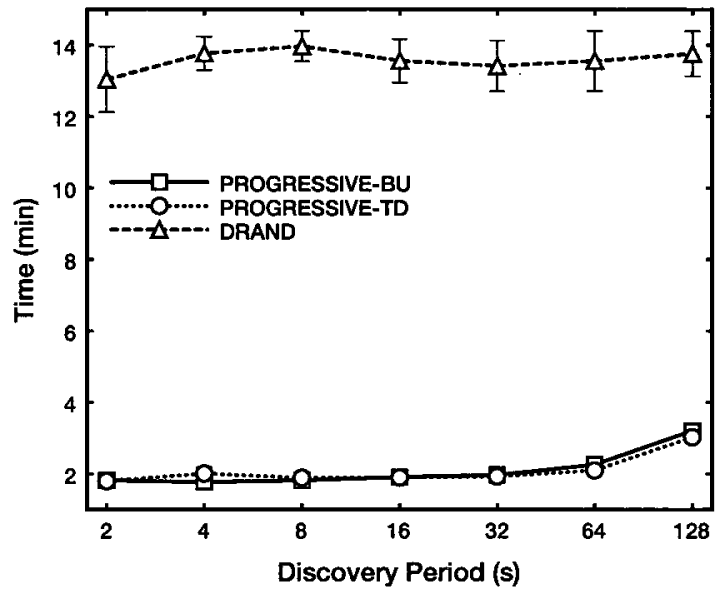

(d) Time

Figure 5.3: Simulation results of PROGRESSIVE and DRAND for different $t_{\text {discovery }}, N$ $=80$. 


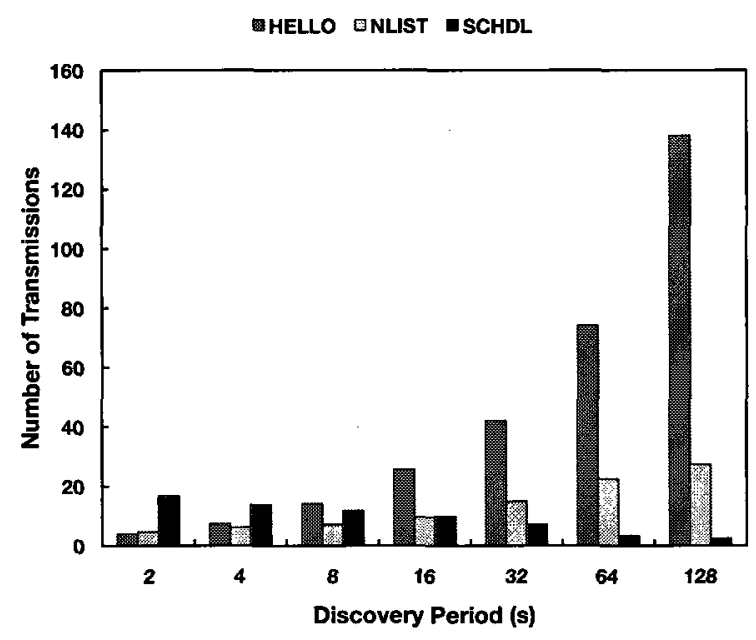

(a) PROGRESSIVE

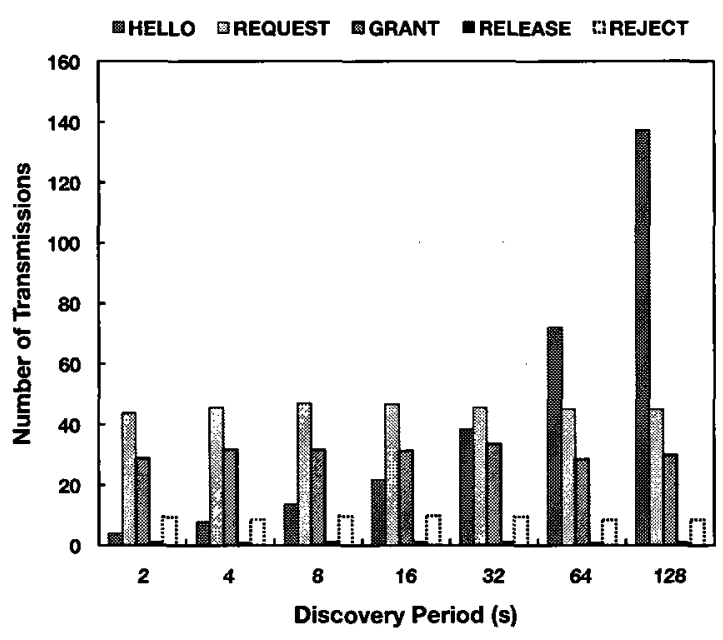

(b) DRAND

Figure 5.4: Number of control message transmissions for different $t_{\text {discovery }}, N=80$.

neighborhood. Despite that, increasing $t_{\text {discovery }}$ results in an increase in the average number of HeLlo messages transmitted. Other types of messages (REQUEST, GRANT, RELEASE, REJECT) are transmitted during the scheduling phase and are not affected by $t_{\text {discovery }}$.

The cost of increasing $t_{\text {discovery }}$ is increasing energy consumption, because of the increase in message transmissions. In addition, as $t_{\text {discovery }}$ increases periods of idle listening increase. These factors are clearly reflected in the energy consumption of PROGRESSIVE scheduling as shown in Figure 5.3(b). In contrast, energy consumption resulting from increasing $t_{\text {discovery }}$ partially contributes to the overall energy consumption of DRAND because much of the energy is consumed during the scheduling phase.

Furthermore, in PROGRESSIVE, the average time spent to achieve $100 \%$ scheduling efficiency increases by $6 \mathrm{~s}$ and $60 \mathrm{~s}$ when $t_{\text {discovery }}$ is $64 \mathrm{~s}$ and $128 \mathrm{~s}$, respectively, 
compared to the time spent when $t_{\text {discovery }}$ is in the range $[2,4,8,16,32] \mathrm{s}$. Unlike PROGRESSIVE, DRAND uses almost the whole scheduling period, 15 minutes, while achieving less than $50 \%$ scheduling efficiency.

\subsubsection{Network Size}

To study the performance of the scheduling protocols for various network sizes, networks of $40,60,80$ and 100 nodes are simulated using $t_{\text {discovery }}=64 \mathrm{~s}$. Figure 5.5 shows simulation results for neighborhood knowledge, energy, scheduling efficiency and time for PROGRESSIVE (with Bottom-Up and Top-Down scheduling) and DRAND.

DRAND maintains $100 \%$ neighborhood knowledge compared to PROGRESSIVE which maintains $98 \%$ neighborhood knowledge falling to $96 \%$ when the network size is 100 nodes. This slight reduction in the PROGRESSIVE's neighborhood knowledge occurs because when the number of nodes increases, the number of hops that NETLIST messages have to traverse also increases. By the end of the topology learning and construction phase some of those NET-LisTs might not be able to reach the sink.

PROGRESSIVE is able to schedule all nodes in all network sizes. DRAND, however, achieves about $88 \%$ scheduling efficiency only when the network has 40 nodes. For larger network size, DRAND's scheduling efficiency continues to fall until it reaches less than $48 \%$ when the network size is 100 nodes.

The energy consumed and the total scheduling time to achieve the previous efficiencies are shown in Figures 5.5(b) and 5.5(d), respectively. DRAND consumes 30 Joules in 10 minutes to schedule 40 nodes. Furthermore, it consumes 40,44 and 45 Joules just to schedule 32 out of 60,35 out of 80 and 39 out of 100 nodes after 
running for $13,13.5$ and 13.75 minutes, respectively. PROGRESSIVE reduces energy consumption by a factor of $9.5,12.8,13.6$ and 13.9 compared to DRAND for networks of 40,60, 100 and 100 nodes, respectively. This performance gain results because PROGRESSIVE scheduling runs for a short duration (less than 3 minutes) and still achieves $100 \%$ scheduling efficiency.

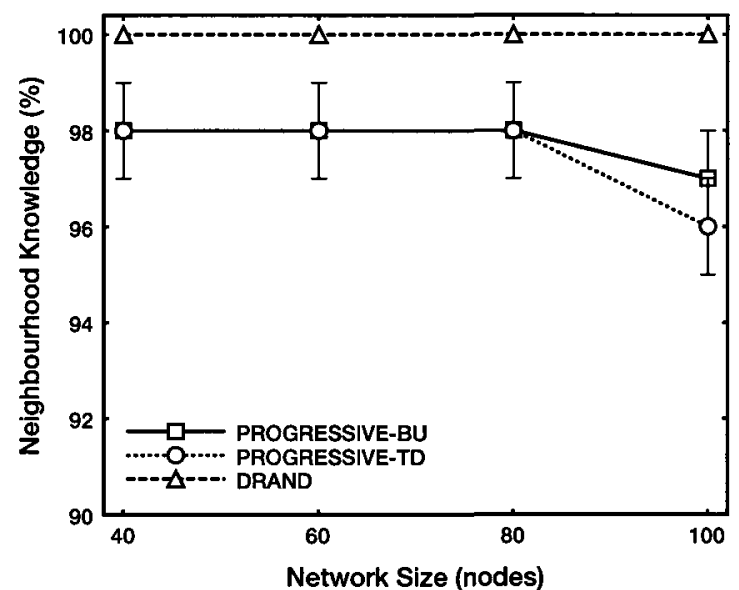

(a) Neighborhood Knowledge

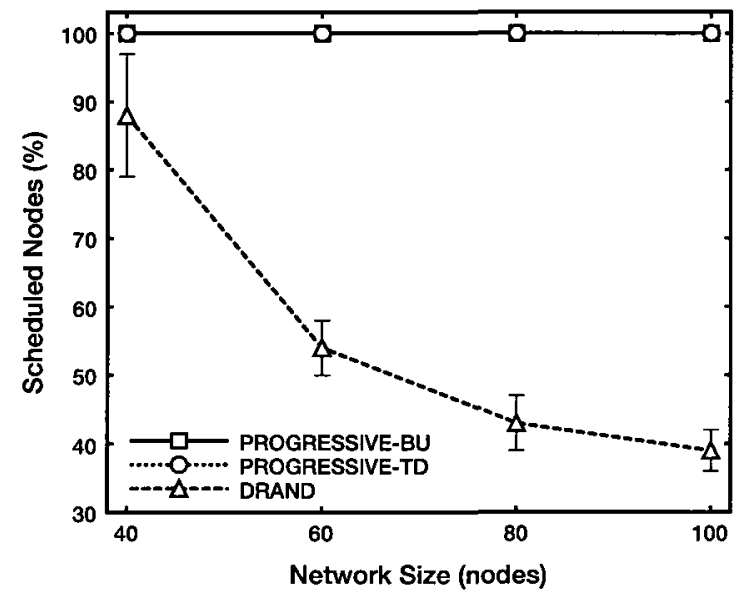

(c) Scheduling Efficiency

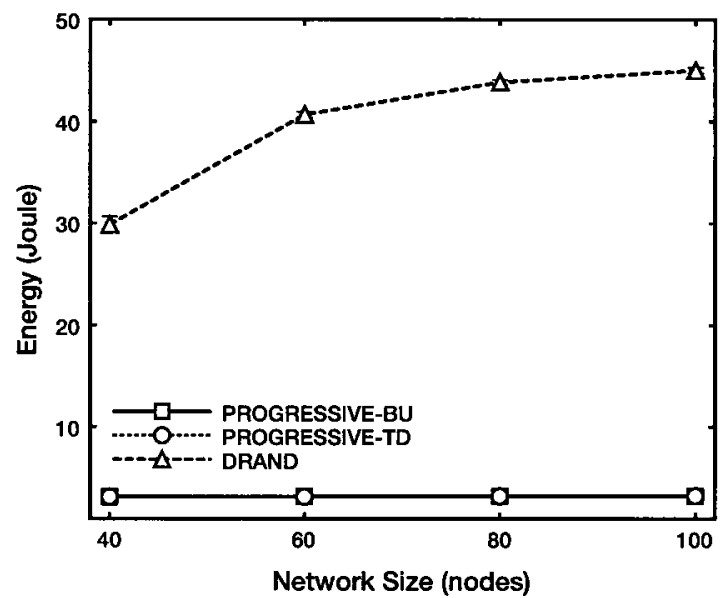

(b) Energy

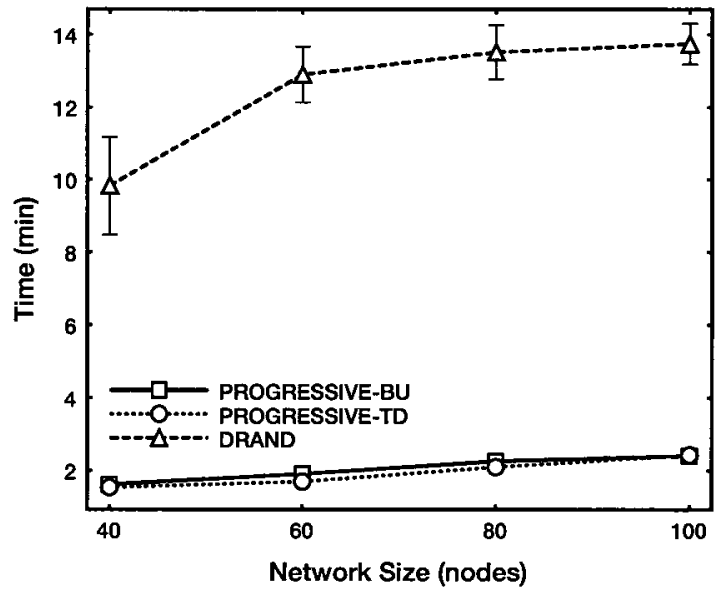

(d) Time

Figure 5.5: Simulation results of PROGRESSIVE and DRAND for different network sizes, $t_{\text {discovery }}=64 \mathrm{~s}$. 
To further demonstrate how PROGRESSIVE outperforms DRAND, a network of 40 nodes is simulated and $t_{\text {discovery }}$ is $16 \mathrm{~s}$. This duration is short enough to show how energy is gradually consumed with time. In Figure 5.6, the energy consumption of both PROGRESSIVE and DRAND increases rapidly during the neighborhood discovery and topology learning phases. This increase happens because both protocols rely on CSMA to exchange control messages among neighbors. However, once the topology learning phase is over each protocol follows a different mechanism to perform the slot assignment process. DRAND continues to use CSMA and all nodes must remain active waiting to receive or transmit a REQUEST, GRANT, RELEASE, or REJECT message. This causes the continuing rapid increase in energy consumption. On the other hand, nodes in PROGRESSIVE start to follow a schedule where they are active during specific time slots and sleep the rest of the time. Unscheduled nodes can sleep, and only wake-up to transmit notifications when their neighbors are receiving. Eventually, notification messages reach the sink and a new schedule is computed.

\subsubsection{Relative Network Density}

Relative network density refers to the average number of two-hop neighbors of a node. This measure is chosen for comparison because it is one of the factors that affect DRAND's performance, since its slot assignment ensures that all two-hop neighbors of a node must be assigned a different slot. To study the relative network density, a network of 100 nodes is simulated. The average number of two-hop neighbors is varied in the range [5-25] in steps of 5. $t_{\text {discovery }}$ is $64 \mathrm{~s}$.

Figure 5.7 shows that neighborhood knowledge and scheduling efficiency are high when the network relative density is 5 . In addition both PROGRESSIVE and DRAND 


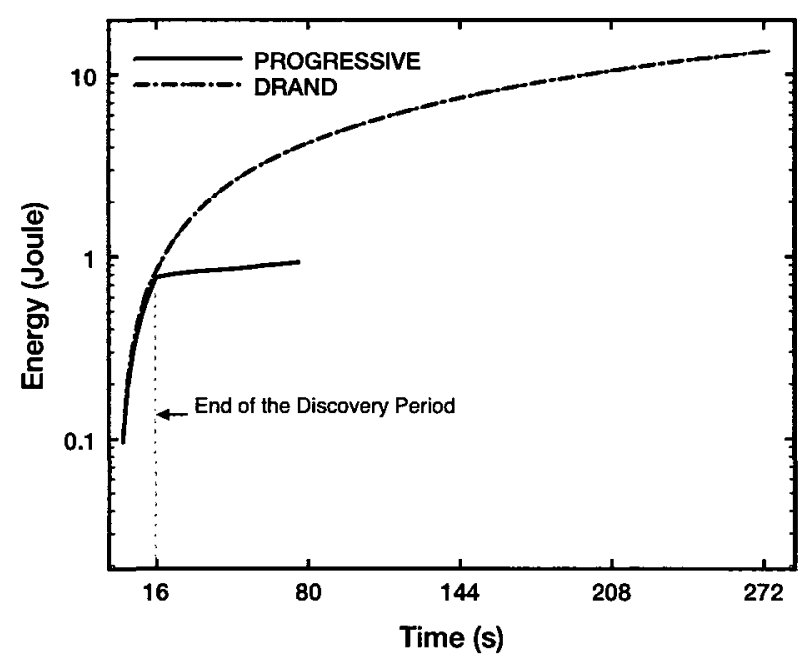

Figure 5.6: Gradual energy consumption of DRAND and PROGRESSIVE, $N=40$, $t_{\text {discovery }}=16 \mathrm{~s}$. PROGRESSIVE schedules all 40 nodes in about $80 \mathrm{~s}$ while DRAND in $272 \mathrm{~s}$. Once $t_{\text {discovery }}$ ends, PROGRESSIVE's energy consumption is more flat compared to DRAND.

consume little energy in a short period of time. However, Figure 5.5(a) shows that as the relative density increases PROGRESSIVE's neighborhood knowledge drops by $6 \%$ to reach $91 \%$ when the relative density is 25 . Nevertheless, Figure 5.5(c) shows that PROGRESSIVE's scheduling efficiency remains at $100 \%$ for all densities. Nodes running DRAND are able to know about all neighbors even at higher densities. However, only $40-50 \%$ of these nodes were able to obtain a transmission slot. This sharp drop in DRAND's scheduling efficiency is due to its slot allocation mechanism, where each node must receive a GRANT message from all its neighbors before it is considered a scheduled node.

Scheduling energy consumption is shown in Figure 5.7(b). It can be observed that the relative density has little effect on PROGRESSIVE energy consumption. In fact, each node consumes 0.66 Joule less energy when the number of two-hop neighbors is 25 compared to what is consumed when the relative density is 5 nodes. This 
occurs because PROGRESSIVE consumes energy the most during the neighborhood discovery and topology learning phases. Message transmissions during these phases are the major cause of energy consumption. NET-LIST messages traverse multihops to reach the sink. Deploying the same number of nodes in a smaller area to increase the relative density leads to a network with a smaller number of hops. Therefore, the average number of hops traversed by a NET-List message is reduced as the relative density increases in the same area. This can be seen in the number of message transmissions in Figure 5.8(a). The number of transmitted NET-LisT messages drop from 23 when the relative density is 5 to 13 when it is 25 . On the other hand, the reduction in neighborhood knowledge increases the number of transmitted ScHDL frames, because fewer NET-Lists results in the sink being more likely to have an incomplete knowledge about the topology. Therefore, more scheduling frames are required to resolve conflicts in the slot assignment.

Increasing relative network density negatively affects DRAND's energy consumption. Figure 5.7(b) shows that by increasing the relative density from 5 to 10 , energy consumption increases by about 30 Joules. The cause for this is similar to the case when increasing the network size, i.e. nodes spend the whole scheduling time exchanging control messages, which are susceptible to collisions and errors. Figure 5.8 (b) shows that the number of REQUEST messages increases sharply when the relative density increases from 5 to 10 nodes. This indicates that most nodes are unable to receive GRANT messages from their neighbors. 


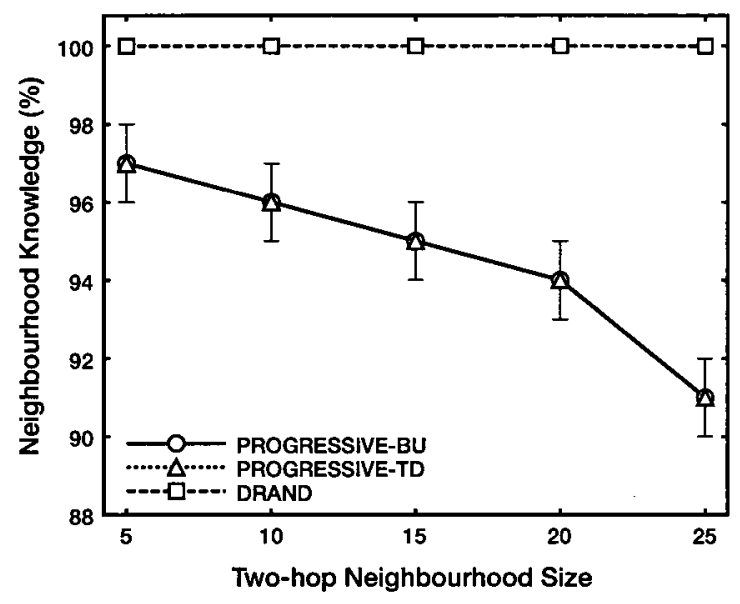

(a) Neighborhood Knowledge

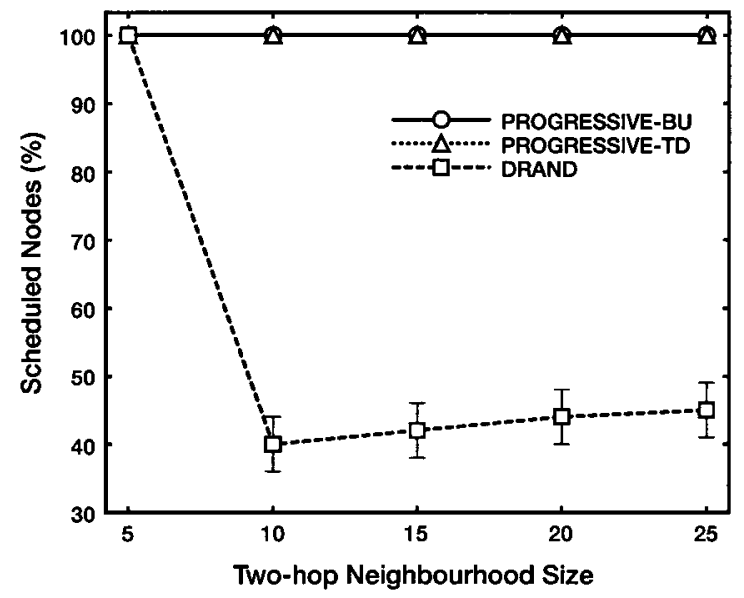

(c) Scheduling Efficiency

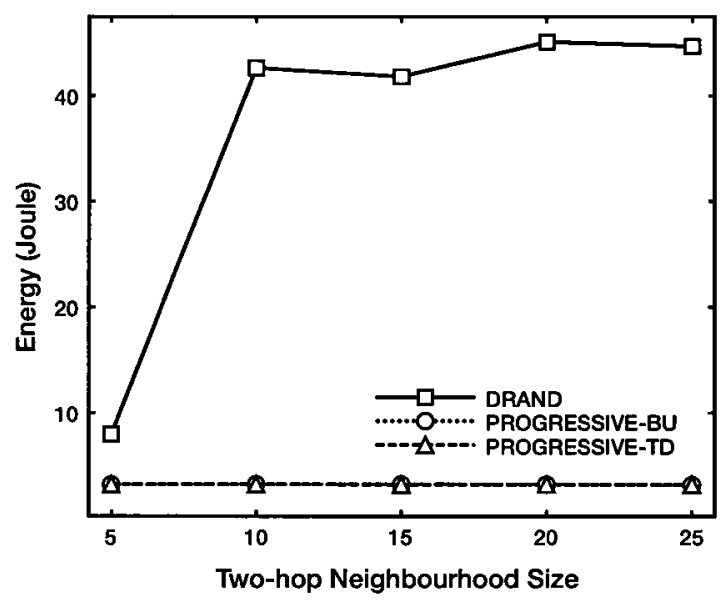

(b) Energy

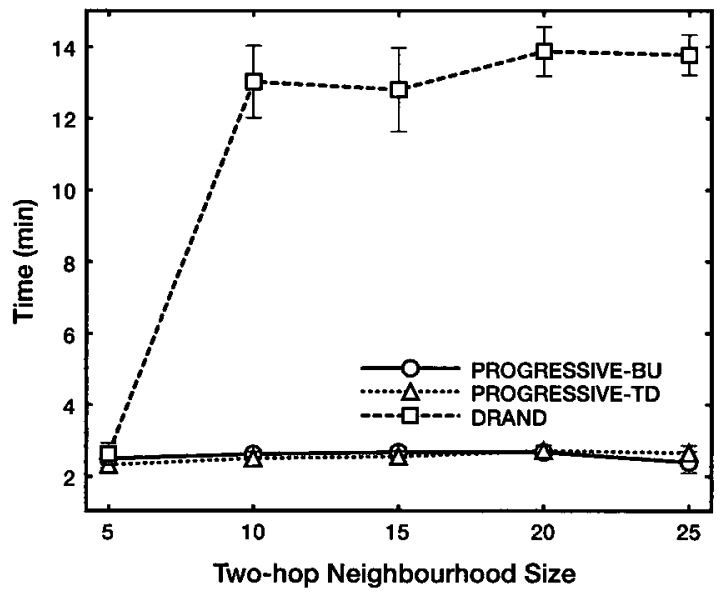

(d) Time

Figure 5.7: Simulation results of PROGRESSIVE and DRAND for different relative network densities, $N=80$, $t_{\text {discovery }}=64 \mathrm{~s}$. 


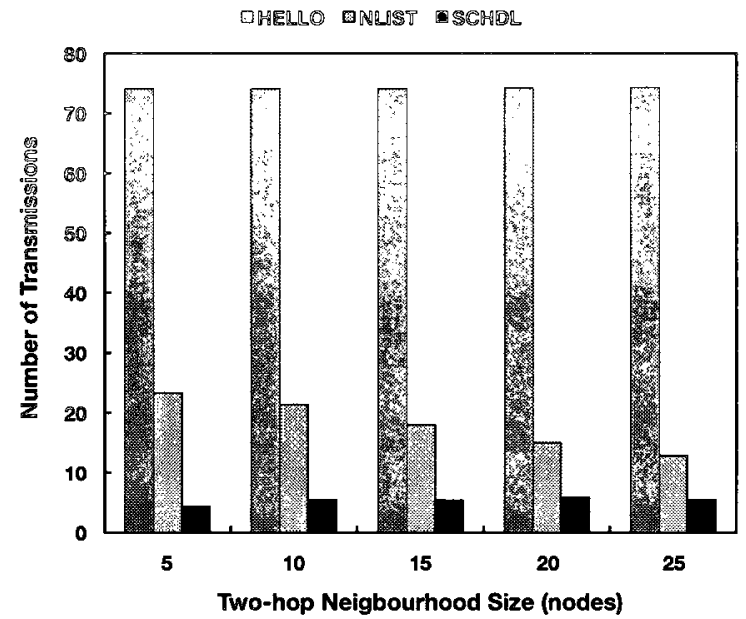

(a) PROGRESSIVE

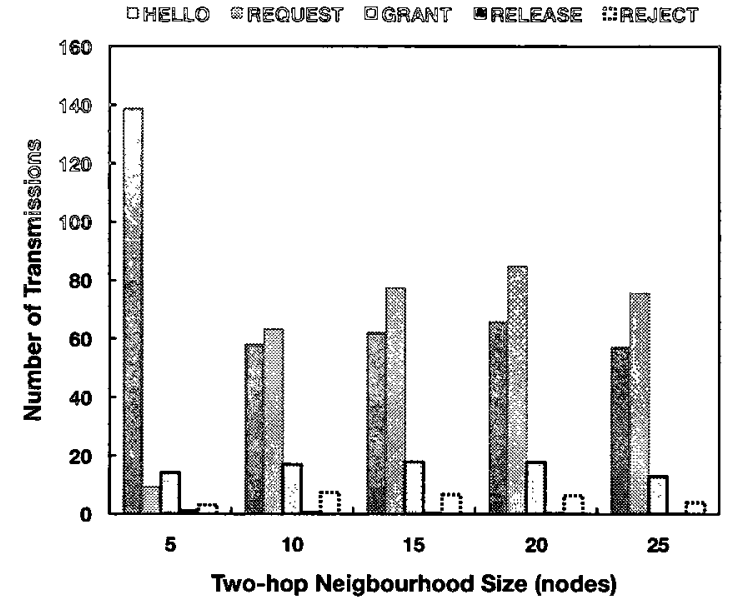

(b) DRAND

Figure 5.8: Number of control message transmissions for different relative network densities, $N=80, t_{\text {discovery }}=64 \mathrm{~s}$.

\subsubsection{Data Rate}

PROGRESSIVE may also be used in ad-hoc networks employing protocols such as 802.11. In such networks, nodes can transmit at higher data rates compared to what is supported in sensor networks. In addition, energy is not a critical resource. Instead, scheduling time and scheduling efficiency are the more important performance metrics. To demonstrate the effect of high data rates, two simulations are conducted: in the first, the network sizes is varied in the range [60 -160] and in the second the network density is varied between [5-45]. A 2Mbps data rate is assumed in both simulations. In previous sections, PROGRESSIVE-BU and PROGRESSIVE-TD have identical results. Therefore, in this section one curve named PROGRESSIVE represents both schemes.

Figure 5.9 shows the scheduling time and efficiency of PROGRESSIVE and DRAND for different network sizes. Indeed, DRAND performs significantly better in high data 
rate networks. It achieves $100 \%$ scheduling efficiency when the number of nodes in the network is up to 160 . For a 100-node network this is an improvement by a factor of 2.5 compared to the efficiency when data rate is $38.4 \mathrm{Kbps}$. Likewise, PROGRESSIVE remains $100 \%$ efficient regardless of the increase in data rate or network size. In both protocols, the time it takes to schedule all nodes in the network increases as the number of nodes in the network increases. However, DRAND needs twice the amount of time as PROGRESSIVE to schedule the same number of nodes.

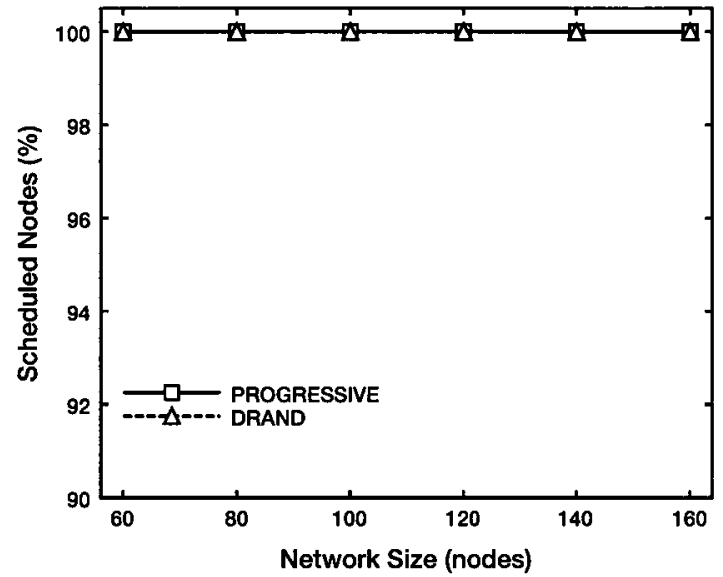

(a) Scheduling Efficiency

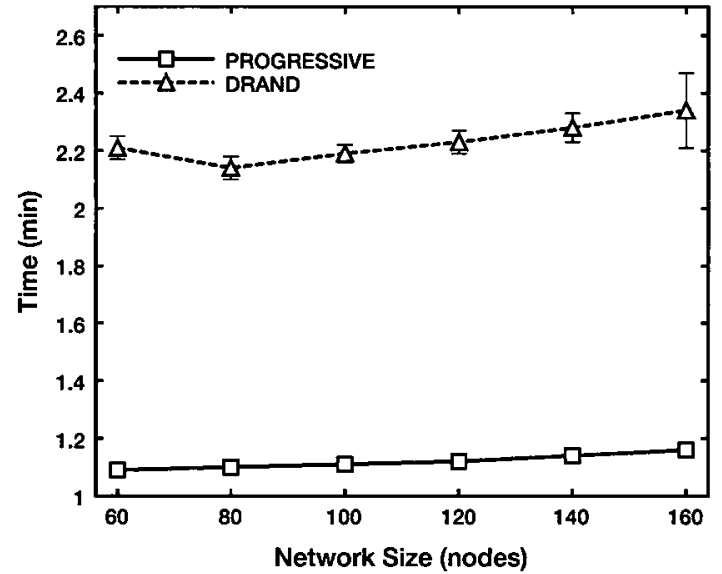

(b) Time

Figure 5.9: Simulation results of PROGRESSIVE and DRAND for different network sizes, data rate $=2 \mathrm{Mbps}$.

Simulation results of PROGRESSIVE and DRAND under variable network densities and a 2Mbps data rate are shown in Figures 5.10 and 5.11. PROGRESSIVE has a superior performance compared to DRAND. It is able to schedule all nodes in the network for all relative network densities. To schedule all nodes when the network density is 45 , PROGRESSIVE requires only $3.5 \%$ of the time spent with DRAND to schedule 82 nodes. In addition, PROGRESSIVE manages to schedule all nodes, while DRAND fails to schedule all nodes once the relative network density is 35 . 


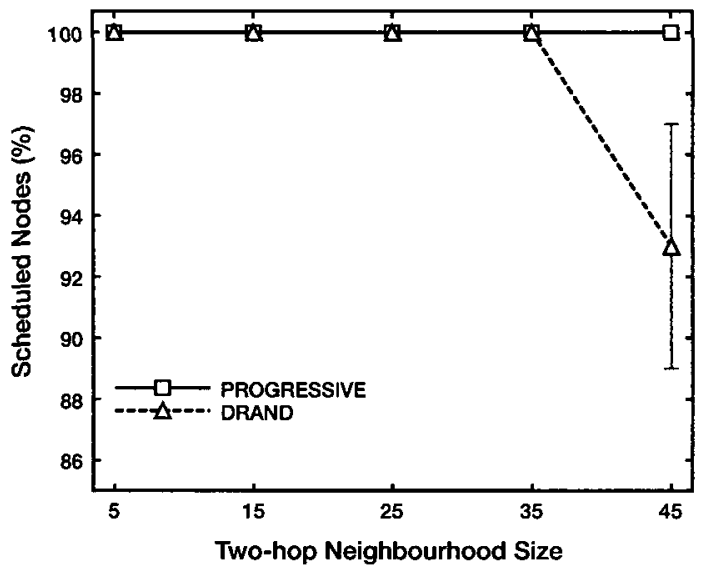

(a) Scheduling Efficiency

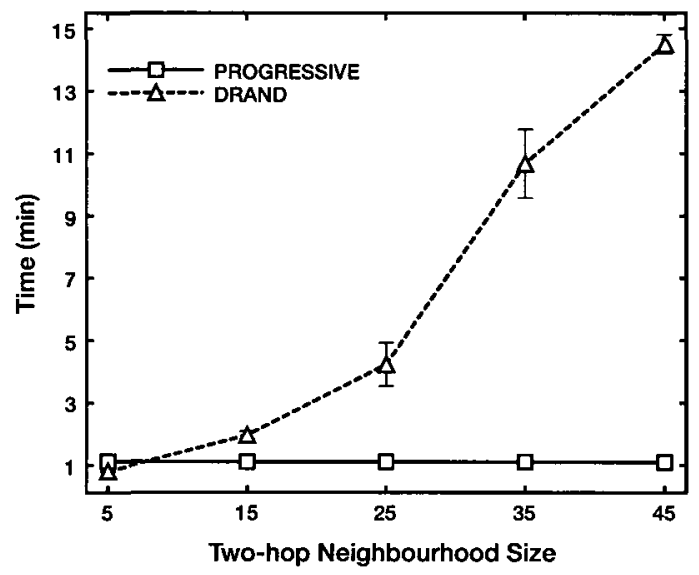

(b) Time

Figure 5.10: Simulation results of PROGRESSIVE and DRAND at different relative network densities, data rate $=2 \mathrm{Mbps}$.

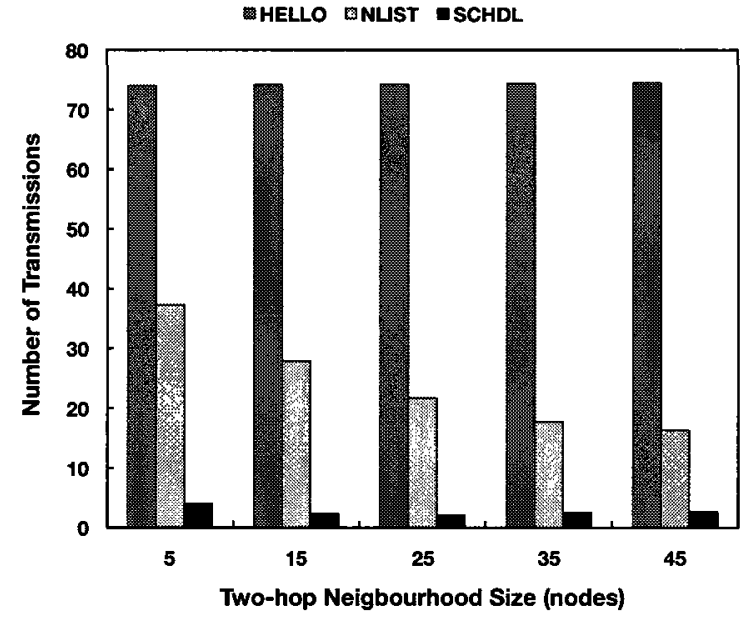

(a) PROGRESSIVE

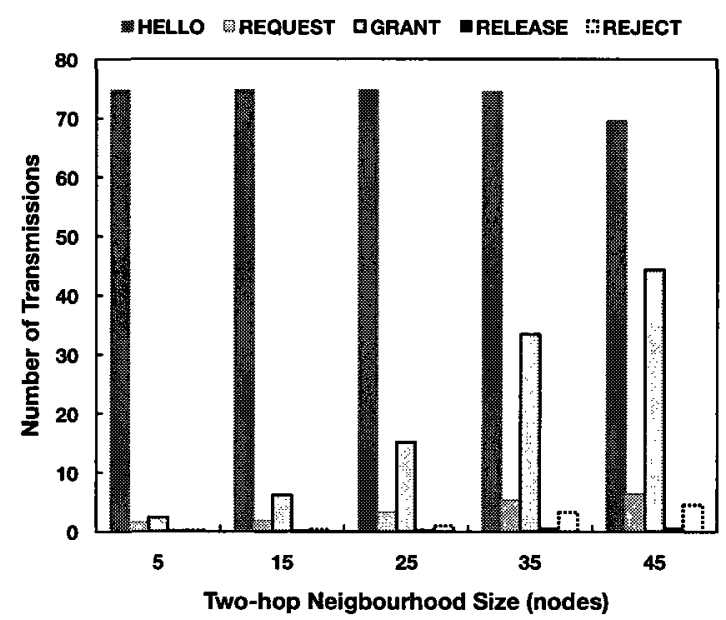

(b) DRAND

Figure 5.11: Number of control message transmissions for different relative network densities, data rate $=2 \mathrm{Mbps}$.

\subsection{OCSMACS Performance}

In this part, OCSMACS simulation results are presented and compared with that of S-MAC, DMAC, Z-MAC and ALWAYS-ON protocols. The protocols are evaluated based on the following performance metrics: 
1. Delay: The end-to-end delay that each packet experiences from the time it is generated until it reaches the sink.

2. Throughput: The total amount of data (bits), excluding overhead, that can be delivered to the sink in $1 \mathrm{~s}$.

3. Energy: The average total energy consumed by each node during the simulation time.

4. Energy Efficiency: The total amount of data (bits) that can be delivered to the sink using $1 \mathrm{~mJ}$ of node's energy.

5. Network Lifetime: The time until the first node in the $1^{\text {st }}$ hop dies.

More detailed performance results, e.g. energy consumed by the radio and the MCU, energy consumed by nodes in different hops, and OCSMACS's scheduling energy overhead are also discussed. These performance metrics are used to evaluate the performance of each protocol for different network sizes and different sampling rates. Similar to PartI, for each simulated metric a total of 20 simulations are carried out, and each simulation runs for 260s using a different random seed. All results are plotted with $95 \%$ confidence intervals. Figures that are plotted in a logarithmic scale are used to clarify the variations in protocols' performance if the results differ by a large margin.

OCSMACS, Z-MAC and S-MAC each has a specific parameter, which is tuned to achieve a specific application requirement. The parameter of OCSMACS is the number of data exchange slots $\mathcal{S}$, for Z-MAC the parameter is the check-interval and for S-MAC it is the duty cycle. For each simulation, the best parameter setting is 
obtained by running a separate set of simulations and the value that gives the best results with a $95 \%$ level of confidence is chosen.

For all simulations, two applications with different requirements are considered: the first application requires that the network operates with the maximum energy efficiency. The second application requires an average end-to-end delay that does not exceed 1s.

\subsubsection{Network Size}

This sub-section presents the performance of these protocols when the network size is varied from [40 - 160] nodes and the sampling rate is $0.1 \mathrm{pkt} / \mathrm{sec}$. The best values of $\mathcal{S}$,

check-interval and duty cycle for the requirements of the two applications are given in Tables 5.3 and 5.4. For the application requiring energy efficiency, OCSMACS-BU is compared with other protocols because it consumes less energy than OCSMACS-TD. For the application requiring no more than 1s delay, we compare with OCSMACSTD since it has a shorter delay. Similarly, the basic DMAC is compared with other protocols concerning energy efficiency. While DMAC with the optional MTS (DMACMTS) is compared for delay minimization since the later employs MTS to minimize delay.

\section{Results Optimized for Energy Efficiency}

Figure 5.12 shows the delay, throughput, energy and energy efficiency of all protocols when the energy efficiency is optimized. It can be observed that OCSMACS-BU, DMAC and ALWAYS-ON protocols experience a continuous increase in the delay as the network size increases. OCSMACS-BU shows a slight increase in delay because 


\begin{tabular}{|c|c|c|c|}
\hline & OCSMACS-BU & Z-MAC & S-MAC \\
\hline \hline $\boldsymbol{N}$ (nodes) & $\mathcal{S}$ (slots) & slot duration (ms) & duty cycle (\%) \\
\hline 40 & 584 & 127 & 10 \\
\hline 60 & 584 & 82 & 20 \\
\hline 80 & 584 & 32 & 20 \\
\hline 100 & 584 & 32 & 30 \\
\hline 120 & 584 & 32 & 30 \\
\hline 140 & 584 & 32 & 50 \\
\hline 160 & 584 & 32 & 50 \\
\hline
\end{tabular}

Table 5.3: Best parameters for OCSMACS-BU, Z-MAC and S-MAC (maximizing energy efficiency), $r_{\text {sampling }}=0.1 \mathrm{pkt} / \mathrm{sec}$.

\begin{tabular}{|c|c|c|c|}
\hline & OCSMACS-TD & Z-MAC & S-MAC \\
\hline \hline $\boldsymbol{N}$ (nodes) & $\mathcal{S}$ (slots) & slot duration (ms) & duty cycle (\%) \\
\hline 40 & 146 & 62 & 10 \\
\hline 60 & 146 & 47 & 20 \\
\hline 80 & 146 & 32 & 20 \\
\hline 100 & 146 & 32 & 30 \\
\hline 120 & 146 & 32 & 30 \\
\hline 140 & 146 & 32 & 50 \\
\hline 160 & 36 & 32 & 50 \\
\hline
\end{tabular}

Table 5.4: Best parameters for OCSMACS-TD, Z-MAC and S-MAC (minimizing delay to a max of 1s), $r_{\text {sampling }}=0.1 \mathrm{pkt} / \mathrm{sec}$. 
the value of $\mathcal{S}$ is the same for all network sizes. The increase can be considered as an increase in the scheduling delay, i.e. an increase in $T_{\text {multihop }}^{B U}$ On the other hand, the effect of choosing different parameter values is reflected on the performance of Z-MAC and S-MAC as a fluctuation in delay. While S-MAC maintains a delay within 1s, Z-MAC's delay increases sharply once the network size is over 80 nodes. DMAC is the most significantly affected protocol when the network size increases while ALWAYS-ON has the best delay among all protocols. The reason for DMAC's poor performance is its problem with spatial contention. Nodes in the same hop wake-up at the same time and compete to transmit to the same neighbor, but only one can be successful. Nodes with unsuccessful transmissions have to wait for the next wakeup interval to carry out the same process.

Similarly, an increase in network size causes an increase in each node's energy consumption. OCSMACS-BU has a superior performance compared to other protocols. It consumes the least energy per node, less than 0.5 Joule, while maintaining a high throughput hence, the best energy efficiency. OCSMACS-BU is characterized by low energy consumption because of its ability to put nodes to sleep for the longest possible period. Moreover, when nodes wake up they do not experience contention and therefore have the least interference during data transmission. ALWAYS-ON is the protocol consuming the most energy because nodes remain in the idle state most of the time. Despite its increase in throughput, it has the worst energy efficiency of all. DMAC consumes less energy than both Z-MAC and S-MAC and it is also more energy efficient. However, its throughput degrades when the network has more than 80 nodes. 


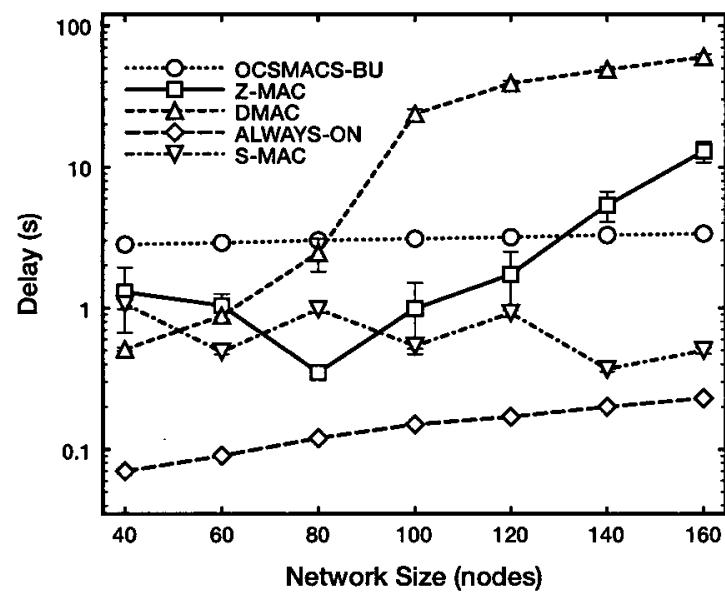

(a) Delay

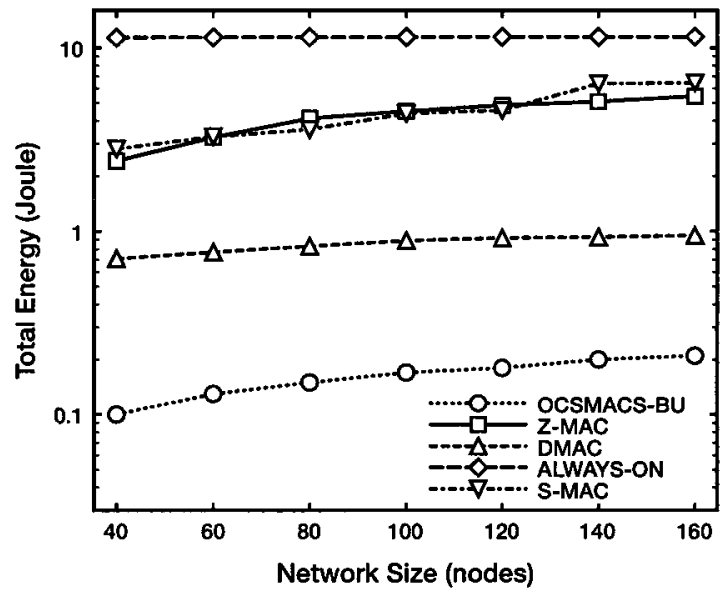

(c) Energy

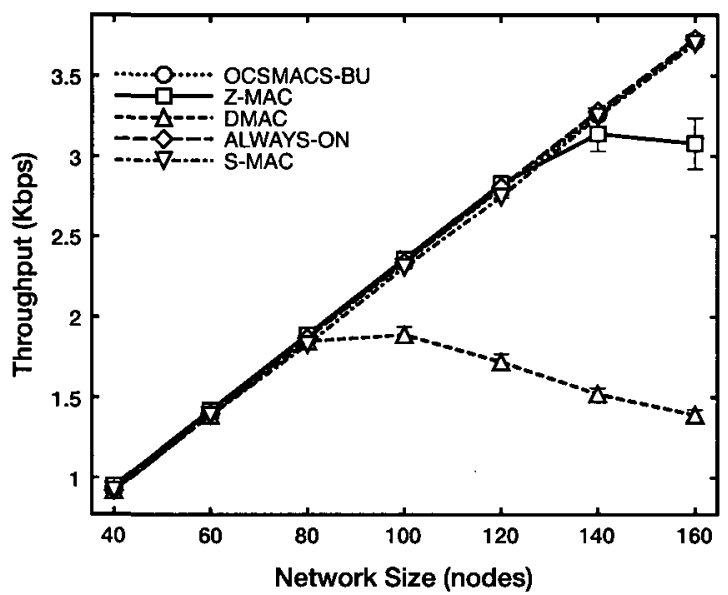

(b) Throughput

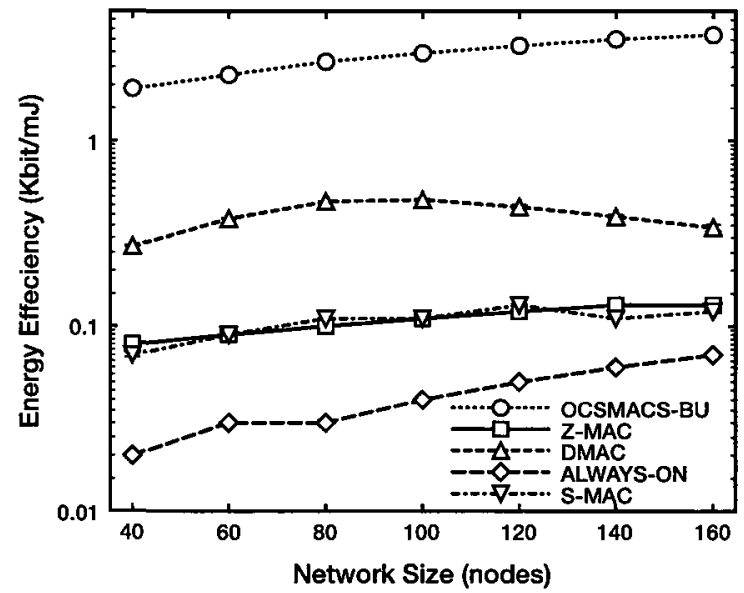

(d) Energy efficiency

Figure 5.12: Simulation results of OCSMACS-BU, Z-MAC, S-MAC, DMAC and ALWAYS-ON for variable number of nodes. OCSMAC, Z-MAC and S-MAC are all optimized to achieve the best energy efficiency for each network size, $r_{\text {sampling }}=0.1 \mathrm{pkt} / \mathrm{sec}$. 
Figure 5.13 shows energy consumption of the radio and the MCU when the network size is 100 nodes. It can be observed that OCSMACS-BU consumes the least energy in transmission, reception and idle listening. The idle listening is the main cause of energy waste in all protocols; it contributes to $10 \%$ of OCSMACS-BU's total energy consumption while it is $29 \%, 33 \%, 35 \%$ and $45 \%$ of DMAC's, Z-MAC's, S-MAC's and ALWAYS-ON's total energy consumption, respectively. The MCU energy, which has been overlooked in past research, is related to radio energy consumption such that the more a node transmits, receives or idle listens the more energy is consumed by the MCU. The figure shows that for OCSMACS-BU, the energy consumption of the MCU is $45 \%$ of a node's total energy consumption. Similarly, the energy consumption of the MCU for Z-MAC or DMAC and S-MAC or ALWAYS-ON is $46 \%$ and $50 \%$ of the total energy consumption, respectively.

The energy consumption and the effective duty cycle of nodes at different hops in the network is important in showing how each protocol balances activity among nodes at different hops. Figure 5.14 shows that the energy consumption and the effective duty cycle increase as the hop count decreases. OCSMACS-BU is the most energy efficient protocol and has the shortest duty cycle compared to other protocols. Its performance is explained by the use of an efficient on-demand slot assignment algorithm. In addition, for each node, OCSMACS keeps track of a counter that indicates how many times this node has been chosen as a receiver. This counter is used to balance energy consumption among all nodes in a specific hop of the network. DMAC also shows better performance compared to Z-MAC, S-MAC and ALWAYSON because of its staggered wakeup. 


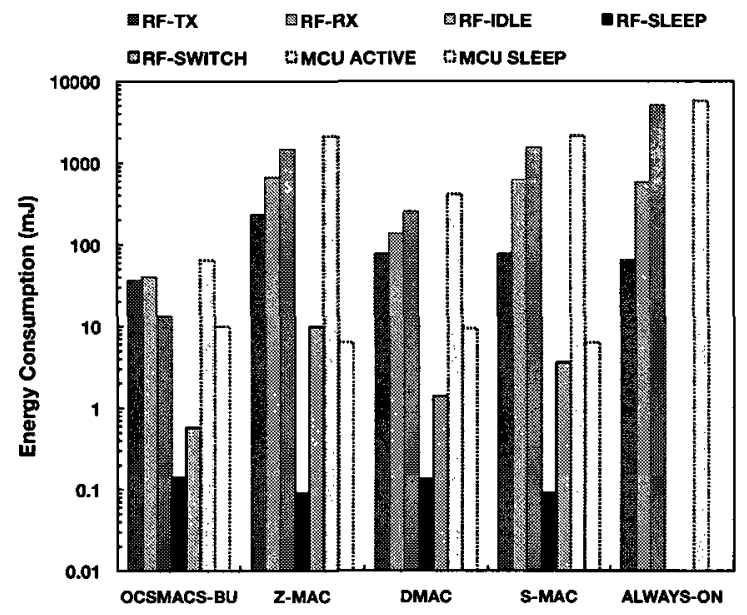

(a) Energy

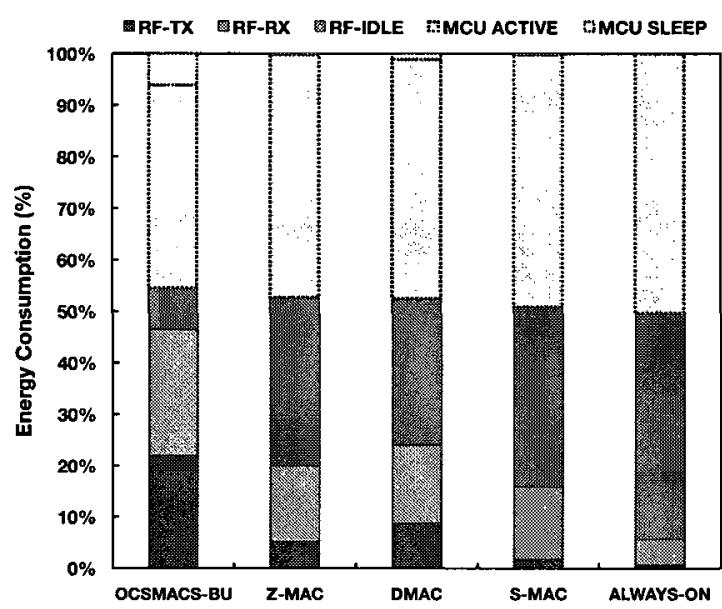

(b) Percentages of total energy

Figure 5.13: Energy consumed by the radio and the MCU during different modes of operation, $N=100, r_{\text {sampling }}=0.1 \mathrm{pkt} / \mathrm{sec}$, optimized for energy efficiency.

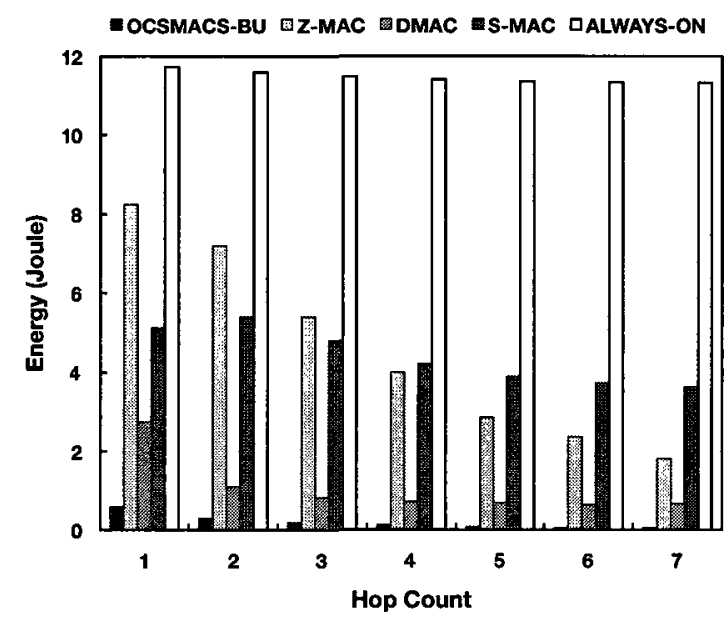

(a) Energy

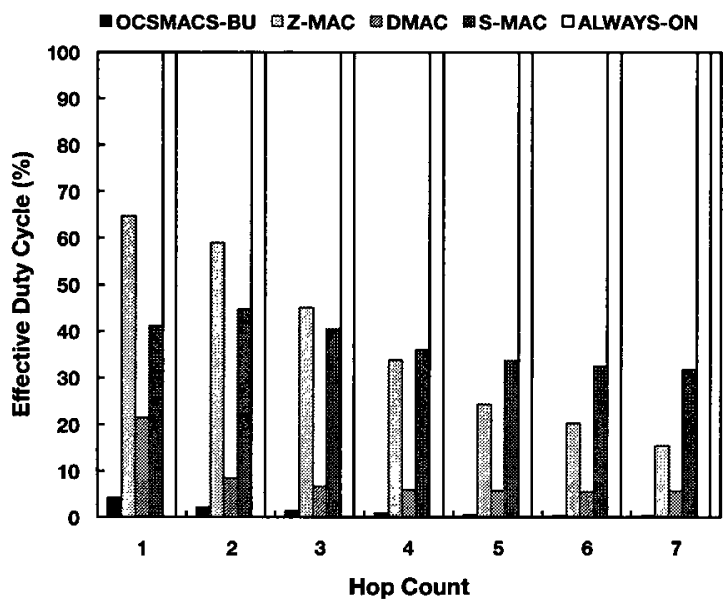

(b) Effective hop duty cycle

Figure 5.14: Energy consumed by nodes at different hops, $N=100, r_{\text {sampling }}=0.1$ $\mathrm{pkt} / \mathrm{sec}$, (optimized for energy efficiency).

It is worth mentioning that even though DMAC and S-MAC duty cycles were preset at $10 \%$ and $30 \%$, respectively, the effective duty cycle of nodes at different hops differ considerably from the preset values. In case of DMAC, the effective duty cycle of the first hop is about $20 \%$ but it falls to less than $10 \%$ for larger hops. With 
respect to S-MAC, a $30 \%$ effective duty cycle can only be noticed at the $7^{\text {th }}$ hop, but it is higher than $30 \%$ at smaller hop counts.

OCSMACS's scheduling overhead energy is important in evaluating the cost of the OCSMACS centralized scheduling scheme. Figure 5.15 shows energy consumption of each node as a result of receiving ScHDL frames and exchanging schedule requests when optimal energy efficiency is required by the application. The trend increases as the network size increases. This is due to the increase in the size of the SchDL frame that carries the slot assignment for all nodes. It is also noteworthy that the energy overhead associated with scheduling is more in OCSMACS-BU compared to that in OCSMACS-TD. Because the energy consumption of OCSMACS-BU is lower, the overhead is more.

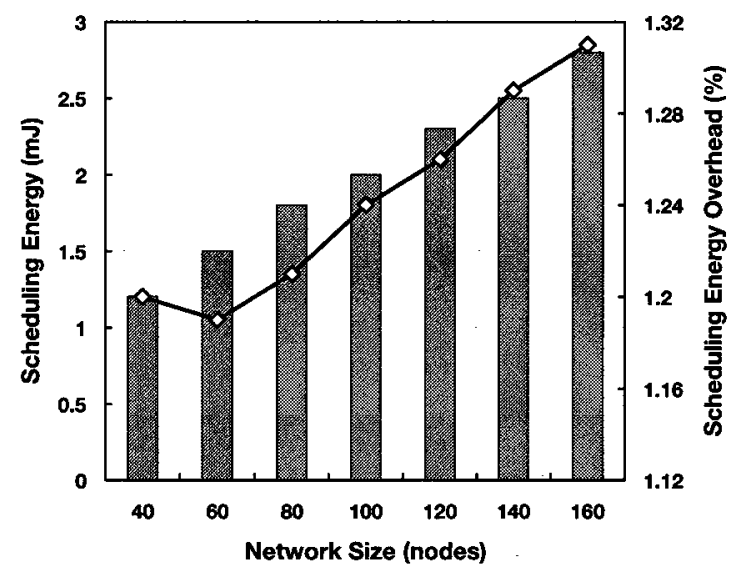

(a) OCSMACS-BU

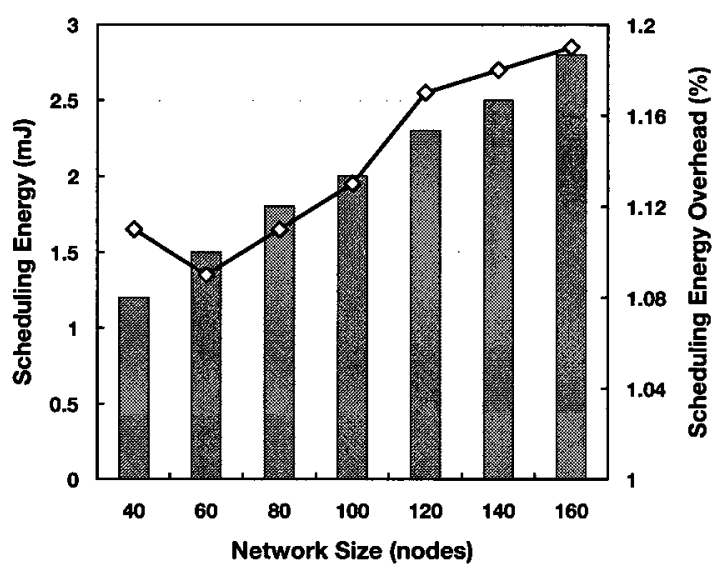

(b) OCSMACS-TD

Figure 5.15: Scheduling energy and its overhead, $r_{\text {sampling }}=0.1 \mathrm{pkt} / \mathrm{sec}$ (optimized for energy efficiency). 


\section{Results Optimized for a 1s Maximum Delay}

When minimizing delay up to a ceiling of $1 \mathrm{~s}$, each protocol does so using the minimum amount of energy. Results for the simulations are shown in Figure 5.16. OCSMACSTD, S-MAC and ALWAYS-ON were all able to achieve the 1s delay objective for all network sizes. However, Z-MAC's delay exceeds that boundary when the network size is more than 100 nodes. Similarly, DMAC-MTS achieves the 1s delay objective when the number of nodes is 80 or less. Beyond this point, DMAC-MTS's delay increases sharply to reach about $45 \mathrm{~s}$ when the network size is 160 . Despite that, DMAC-MTS has a better delay than the basic DMAC when the network size is 80 .

The energy consumption and energy efficiency of Z-MAC and S-MAC do not change from those presented in Figure 5.12 since the optimal parameter settings are the same in those cases. However, a 1s maximum delay requires OCSMACS-TD to reduce $\mathcal{S}$ from 584 to 146 for all network sizes, except when there are 160 nodes in which case $\mathcal{S}$ is set to 36 . The drawback of having a small value of $\mathcal{S}$ is that the frequency of ScheduleReq and ResP/Sync slots increases. Hence, nodes wake up more often and more energy is consumed. Reducing the frame size causes an increase in the energy consumption of OCSMACS-TD by a factor of one compared to OCSMACS-BU, when optimized for energy efficiency.

Another effect related to increasing the frequency of the SCHEDULEREQ and RESP/SynC slots is an increase in the scheduling energy overhead (see Figure 5.17). For OCSMACS$\mathrm{TD}$, changing $\mathcal{S}$ from 584 to 146 is only required when the network has 160 nodes. In this case, about $38 \%$ of a node's energy is wasted on the scheduling process. On the other hand, to maintain the 1 s maximum delay requirement, OCSMAC-BU requires 
smaller $\mathcal{S}$ starting from a network size of 120 nodes. OCSMACS-BU scheduling energy overhead is 163 times and 158 times that of OCSMAC-TD when the network size is 120 and 140 , respectively.

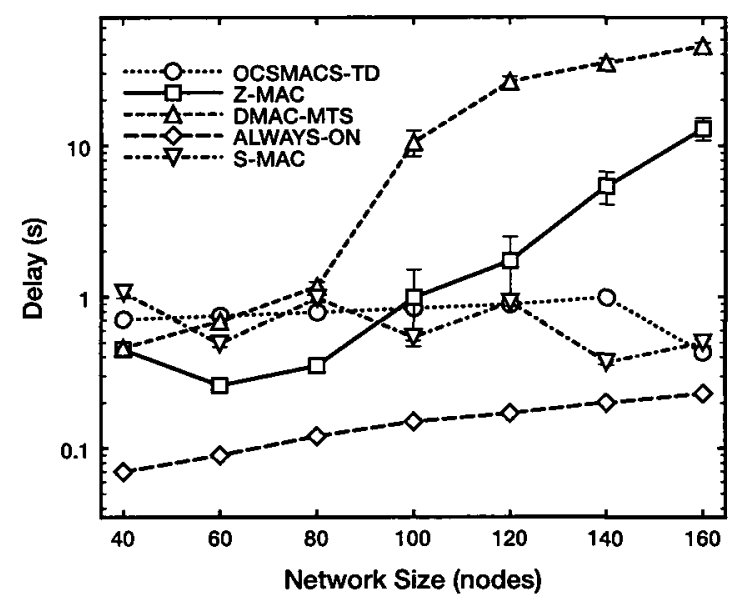

(a) Delay

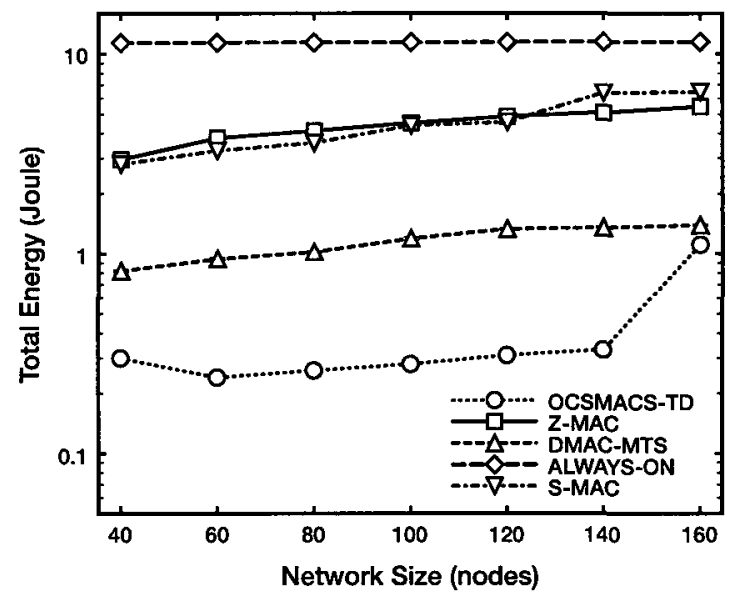

(c) Energy

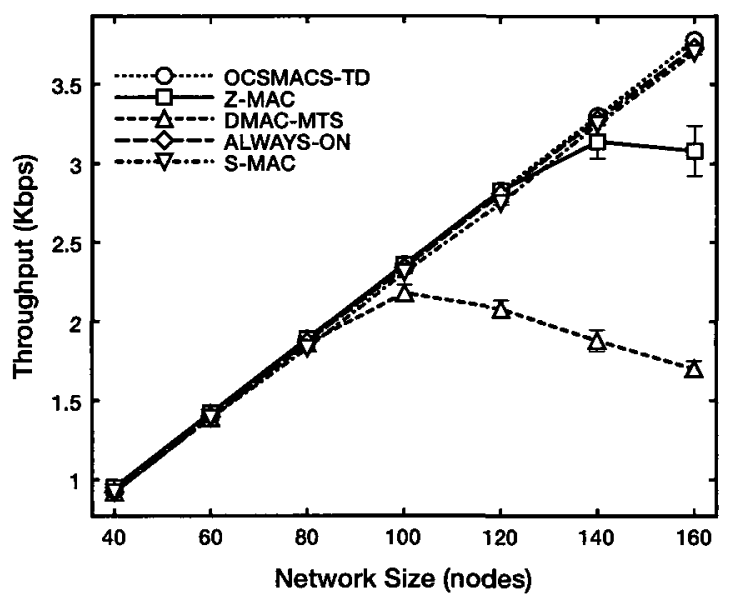

(b) Throughput

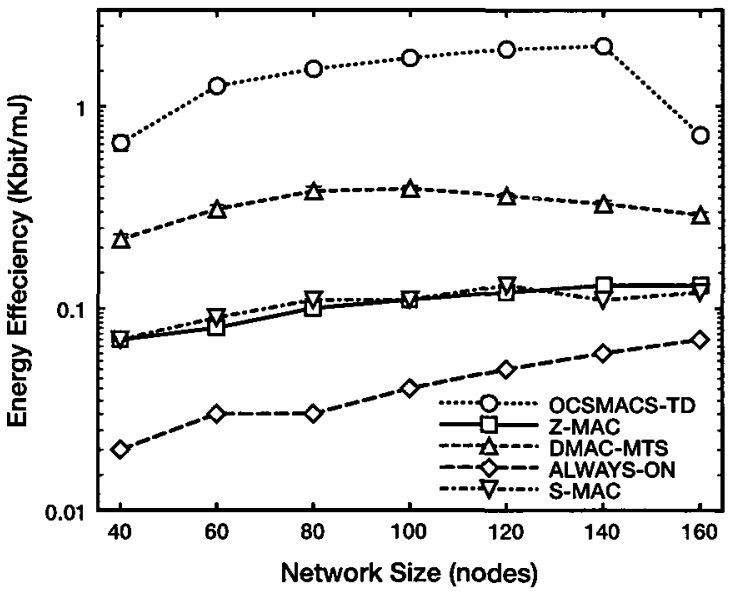

(d) Energy efficiency

Figure 5.16: Simulation results of OCSMACS-TD, Z-MAC, S-MAC, DMAC and ALWAYS-ON for variable number of nodes. OCSMAC, Z-MAC and S-MAC are all optimized to achieve a $1 \mathrm{~s}$ maximum end-to-end delay. A delay of more than $1 \mathrm{~s}$ means that the protocol cannot achieve this requirement at the specific network size, $r_{\text {sampling }}=0.1$ $\mathrm{pkt} / \mathrm{sec}$. 


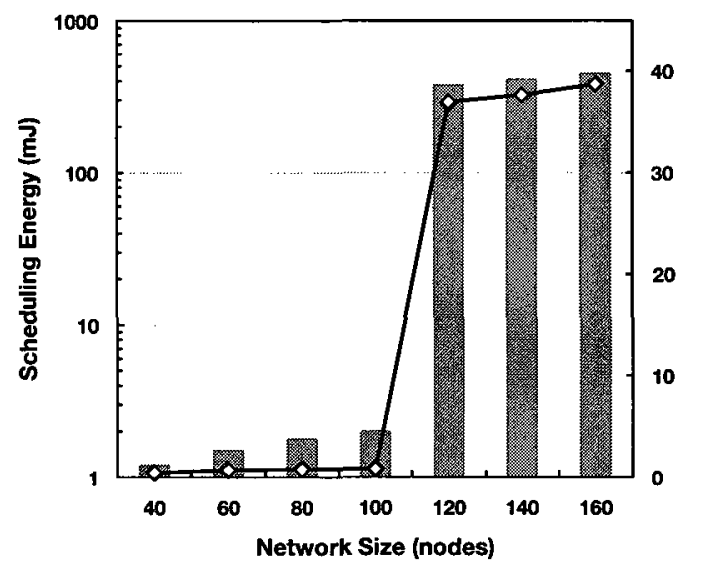

(a) OCSMACS-BU

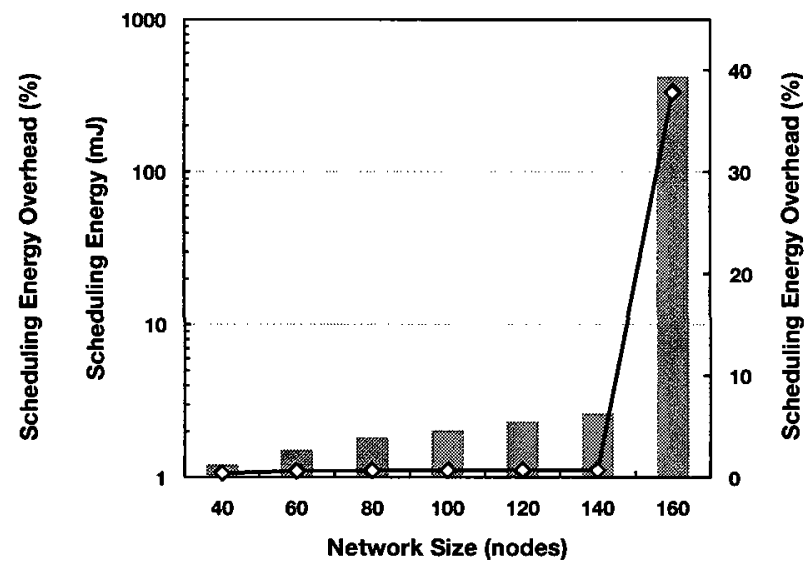

(b) OCSMACS-TD

Figure 5.17: Scheduling energy overhead, $r_{\text {sampling }}=0.1 \mathrm{pkt} / \mathrm{sec}$ (optimized for $1 \mathrm{~s}$ maximum delay).

\section{Network Lifetime}

The objective of this section is to compute network lifetime subject to a maximum $40 \mathrm{~s}$ delay. Networks of [40-160] nodes are simulated assuming a very low sampling rate of $0.003 \mathrm{pkt} / \mathrm{sec}$. In addition, sensors are assumed to carry $2 \mathrm{AA}$ alkaline batteries of $2000 \mathrm{mAh}$. The best values of $\mathcal{S}$, check interval, duty cycle, for OCSMACS, Z-MAC and S-MAC, respectively, are shown in Table 5.5. DMAC and ALWAYS-ON are not considered in this section since neither can be optimized for this low sampling rate; therefore they would perform much worse than other protocols.

Figure 5.18 shows lifetimes of OCSMACS, Z-MAC and S-MAC. It can be observed that OCSMACS has a superior performance compared to Z-MAC and S-MAC. Specifically, when the network consists of 40 nodes, OCSMACS extends network lifetime by 3 years compared to Z-MAC and S-MAC. When the network is 160 nodes, OCSMACS extends lifetime approximately 1 year. This drop in network lifetime results from the fact that nodes that are one hop away from the sink forward more data, as 
the total number of nodes in the network increases. This increase in data forwarding is reflected as an increase in energy consumption and subsequently a decrease in network lifetime.

\begin{tabular}{|c|c|c|c|}
\hline & OCSMACS-BU & Z-MAC & S-MAC \\
\hline \hline $\boldsymbol{N}$ (nodes) & $\mathcal{S}$ (slots) & slot duration (ms) & duty cycle (\%) \\
\hline 40 & 6000 & 1000 & 2 \\
\hline 60 & 6000 & 1000 & 2 \\
\hline 80 & 6000 & 800 & 3 \\
\hline 100 & 6000 & 600 & 3 \\
\hline 120 & 6000 & 400 & 3 \\
\hline 140 & 6000 & 300 & 3 \\
\hline 160 & 6000 & 300 & 3 \\
\hline
\end{tabular}

Table 5.5: Best parameters for OCSMACS-BU, Z-MAC and S-MAC, maximizing lifetime, $r_{\text {sampling }}=0.003 \mathrm{pkt} / \mathrm{sec}, 40 \mathrm{~s}$ maximum delay.

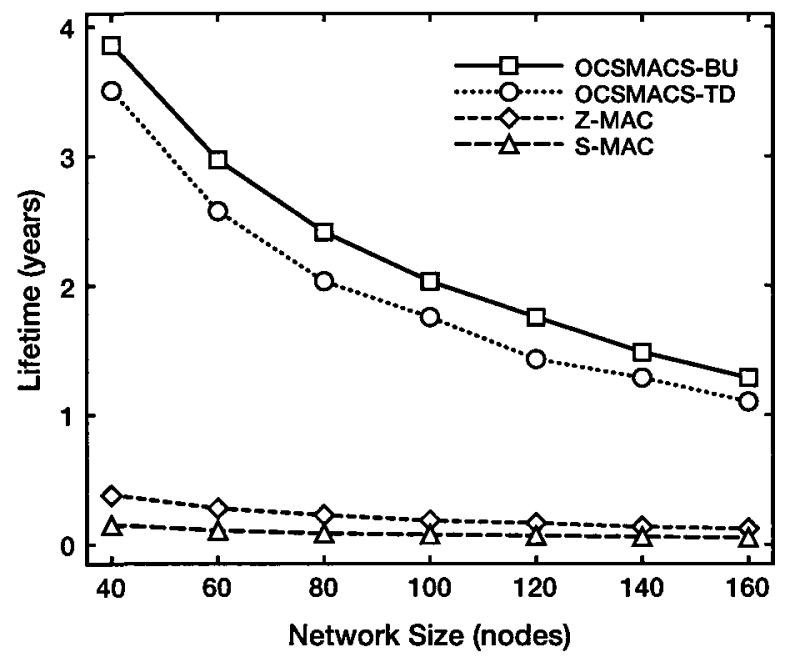

Figure 5.18: Network lifetime, $r_{\text {sampling }}=0.003 \mathrm{pkt} / \mathrm{sec}$.

\subsubsection{Sampling Rate}

This next simulation-based evaluation studies the performance of the protocols when data rate is varied from a moderate $(0.25 \mathrm{pkt} / \mathrm{sec})$ to relatively high $(4 \mathrm{pkt} / \mathrm{sec})$. A 
network of 100 nodes is simulated and 10 sources are chosen at random to generate data at the given sampling rates. Tables 5.6 and 5.7 show the best parameters for OCSMACS, Z-MAC and S-MAC for the two suggested applications, i.e. optimal energy efficiency and 1s maximum delay.

\begin{tabular}{|c|c|c|c|}
\hline & OCSMACS-BU & Z-MAC & S-MAC \\
\hline \hline$r_{\text {sampling }}$ (pkt/sec) & $\mathcal{S}$ (slots) & slot duration (ms) & duty cycle (\%) \\
\hline 0.25 & 292 & 110 & 10 \\
\hline 0.5 & 146 & 47 & 20 \\
\hline 1 & 73 & 37 & 30 \\
\hline 2 & 36 & 32 & 60 \\
\hline
\end{tabular}

Table 5.6: Best parameters for OCSMACS-BU, Z-MAC and S-MAC for variable $r_{\text {sampling, }}$ $N=100,10$ sources, optimized for energy efficiency.

\begin{tabular}{|c|c|c|c|}
\hline & OCSMACS-TD & Z-MAC & S-MAC \\
\hline \hline$r_{\text {sampling } \text { (pkt/sec) }}$ & $\mathcal{S}$ (slots) & slot duration (ms) & duty cycle (\%) \\
\hline 0.25 & 146 & 72 & 10 \\
\hline 0.5 & 73 & 47 & 20 \\
\hline 1 & 36 & 37 & 30 \\
\hline 2 & 18 & 32 & 60 \\
\hline
\end{tabular}

Table 5.7: Best parameters for OCSMACS-TD, Z-MAC and S-MAC for variable $r_{\text {sampling, }}$ $N=100,10$ sources, optimized for 1 s maximum delay.

\section{Results Optimized for Energy Efficiency}

Simulation results are shown in Figure 5.19, from which it can be observed that optimizing the energy efficiency results in a unified trend in all protocols. For example, OCSMACS-BU and S-MAC maintain an end-to-end delay less than 3s for all sampling rates. Z-MAC has a similar performance but its delay reaches up to $15 \mathrm{~s}$ at $2 \mathrm{pkt} / \mathrm{sec}$ sampling rate. The problem with Z-MAC is that, as the sampling rate increases, the number of contending nodes on a given slot increases, and only one node can 
successfully transmit. Other nodes need to delay their transmissions and contend for another slot. When an owner of a slot experiences contention for its slots, ECN messages are used to relieve that contention. However, ECN messages are susceptible to collisions too. On the other hand, DMAC's delay continues to increase from 0.4s at $0.25 \mathrm{pkt} / \mathrm{sec}$ until it reaches $50 \mathrm{~s}$, the highest among all, at $2 \mathrm{pkt} / \mathrm{sec}$. As previously discussed, DMAC's poor performance is due to its spatial contention and delayed staggered wakeup scheme. If nodes fail to transmit in the current wake-up period, they have to wait long periods before attempting to access the channel.

Unlike our results for delay, all protocols, except ALWAYS-ON, show a gradual increase in their energy consumption as the sampling rate increases. However, at a sampling rate of $0.25 \mathrm{pkt} / \mathrm{sec}$, OCSMACS-BU consumes $76 \%, 81 \%, 91 \%$ and $99 \%$ less energy than DMAC, Z-MAC, S-MAC and ALWAYS-ON, respectively. Similarly, at a $4 \mathrm{pkt} / \mathrm{sec}$ rate, it consumes $43 \%, 88 \%, 92 \%$ and $95 \%$ less energy than DMAC, Z-MAC and ALWAYS-ON. These energy savings are reflected on OCSMACS-BU energy efficiency. The figure illustrates that when the sampling rate is $0.25 \mathrm{pkt} / \mathrm{sec}$, it transports $1.4 \mathrm{Kbits}$ of data consuming $1 \mathrm{~mJ} /$ node. This is an improvement by a factor of more than 7 compared to other protocols. High efficiency $(1.6 \mathrm{Kbit} / \mathrm{mJ})$ is also achieved at $2 \mathrm{pkt} / \mathrm{sec}$ sampling rate. Both Z-MAC's and DMAC's throughput drops when sampling rate is $2 \mathrm{pkt} / \mathrm{sec}$. As a result, their energy efficiency drops. On the other hand, S-MAC and ALWAYS-ON maintain the same throughput as OCSMAC-BU but they consume more energy; therefore, they are less energy efficient. 


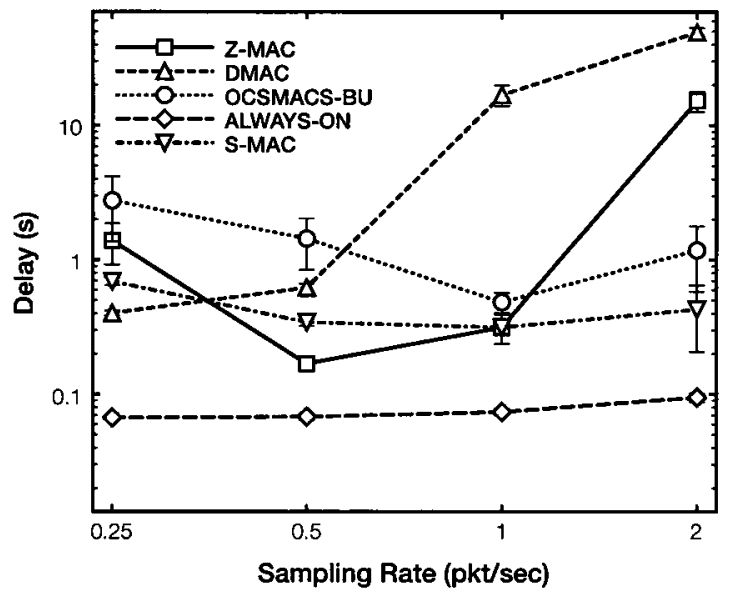

(a) Delay

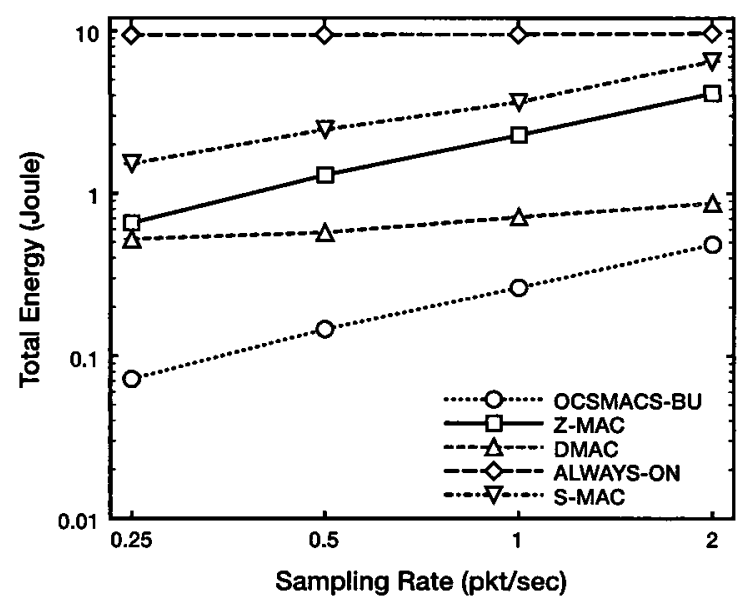

(c) Energy

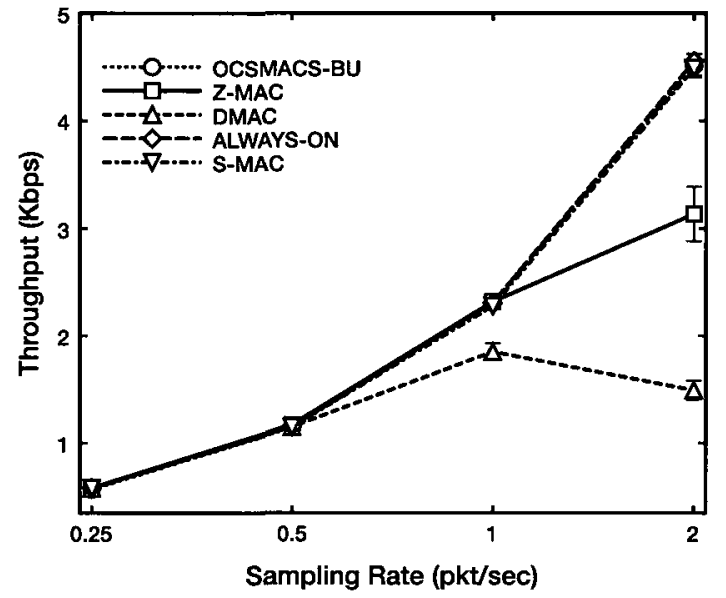

(b) Throughput

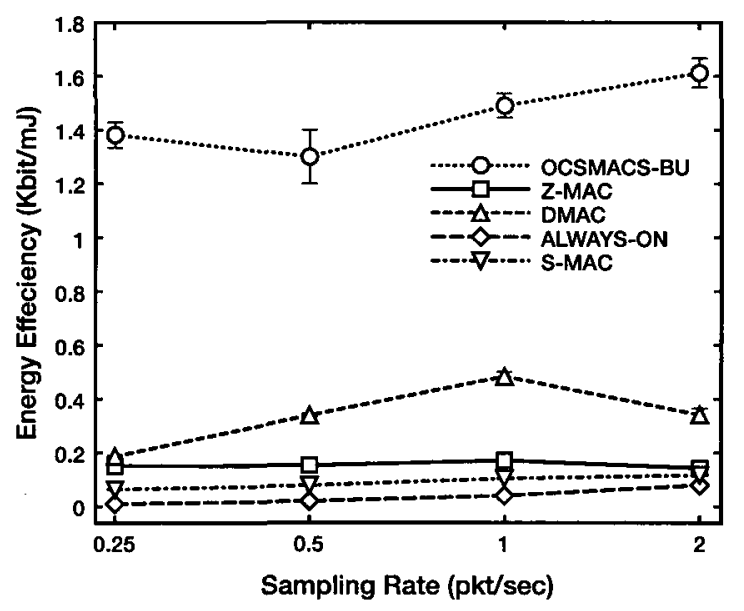

(d) Energy efficiency

Figure 5.19: Simulation results of OCSMACS-BU, Z-MAC, S-MAC, DMAC and ALWAYS-ON for variable sampling rates, $N=100,10$ sources (optimized for energy efficiency). 


\section{Results Optimized for a 1s Maximum Delay}

The simulation results for an application requiring a 1s maximum end-to-end delay are shown in Figure 5.20. It is interesting to notice that in the case of OCSMAC-TD and S-MAC, keeping the delay within 1s requires small values for OCSMAC-TD's $\mathcal{S}$ and S-MAC's duty cycle. The chosen values lead to a reduction in delay even when the sampling rate increases. Z-MAC follows the same trend, however, at $4 \mathrm{pkt} / \mathrm{sec}$ the check interval does not handle the increase in contention. Hence, under Z-MAC the delay increases sharply. With DMAC-MTS, the delay increases as the sampling rate increases. For 1 and $2 \mathrm{pkt} / \mathrm{sec}$ sampling rates, its delay is $10 \mathrm{~s}$ less than that of DMAC.

Increasing the sampling rate increases the number of data packets transmitted. Therefore, energy consumption increases. This is the case for all protocols with the exception of ALWAYS-ON, in which idle listening is the primary cause of energy consumption. Despite being the least energy-consuming and the most energy-efficient protocol, OCSMACS-TD raises energy consumption and reduces efficiency by a factor of 1.6 compared to OCSMACS-BU when the latter is optimized for energy efficiency. It can be concluded from Figure 5.20 and 5.21 that the break point for Z-MAC and DMAC is the $2 \mathrm{pkt} / \mathrm{sec}$ sampling rate. In other words, once the sampling rate is 2 $\mathrm{pkt} / \mathrm{sec}$ it costs more energy to deliver one Kbit of data to the sink than at lower sampling rates. Furthermore, as the sampling rate increases OCSMACS consumes less energy to deliver one Kbit of data to the sink because a higher data delivery to the sink compensates for the energy overhead caused by scheduling.

Figure 5.22 demonstrates how each protocol balances the load among nodes at different hops, e.g. relaying traffic and duty cycling. OCSMACS and DMAC show 


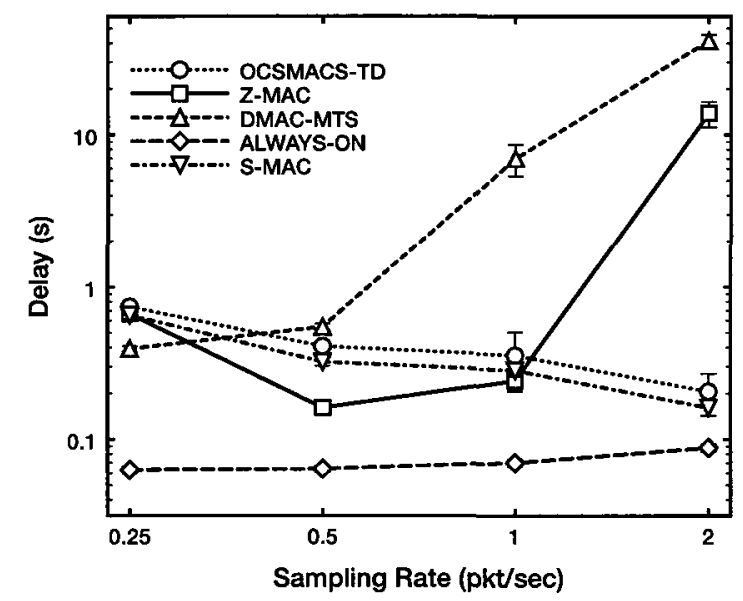

(a) Delay

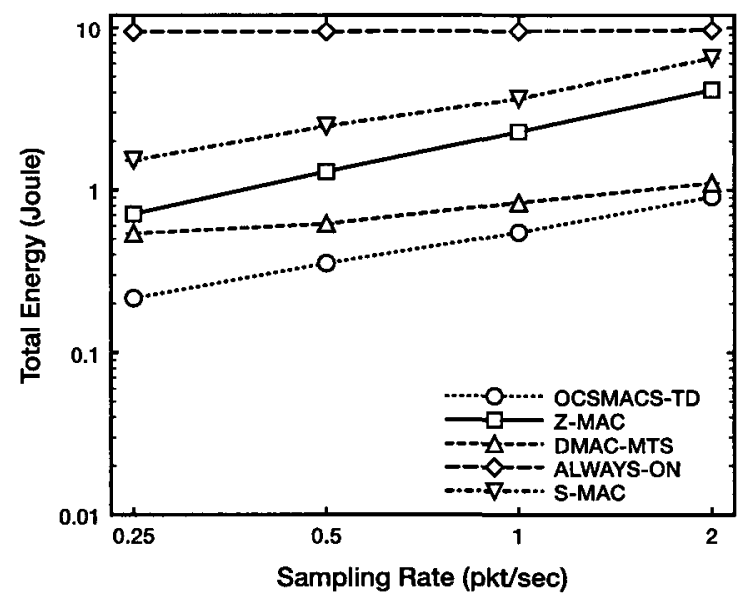

(c) Energy

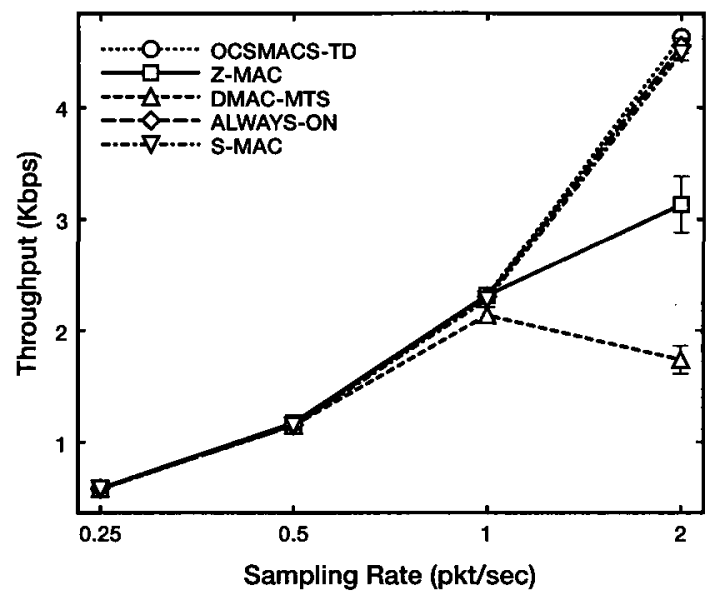

(b) Throughput

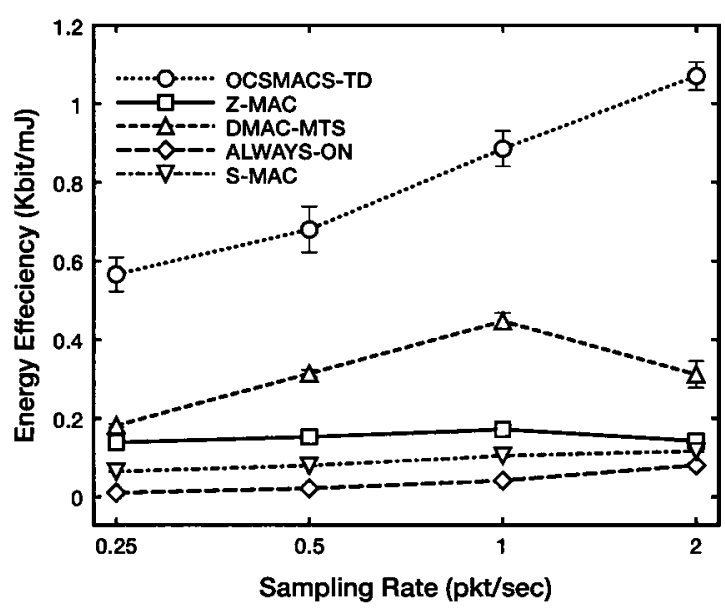

(d) Energy efficiency

Figure 5.20: Simulation results of OCSMACS-TD, Z-MAC, S-MAC, DMAC and ALWAYS-ON for variable number of nodes, $N=100,10$ sources. For each sampling rate, OCSMAC, Z-MAC and S-MAC specific parameters are optimized for 1s maximum end-toend delay. A delay of more than 1s means the protocol cannot achieve this requirement given the specific sampling rate. 
a similar trend in balancing the load among nodes. This is because both protocols employ the same mechanism that specifies wake-up times according to the relative position of nodes in the network. OCSMACS outperforms DMAC because it keeps track of how many times each node is selected as an upstream receiver. This information helps the scheduler in choosing nodes that have been selected the least number of times. On the other hand, Z-MAC energy consumption significantly varies among nodes at different hops, because in Z-MAC all nodes have the same check interval regardless of whether they are close to the sink or far from it.

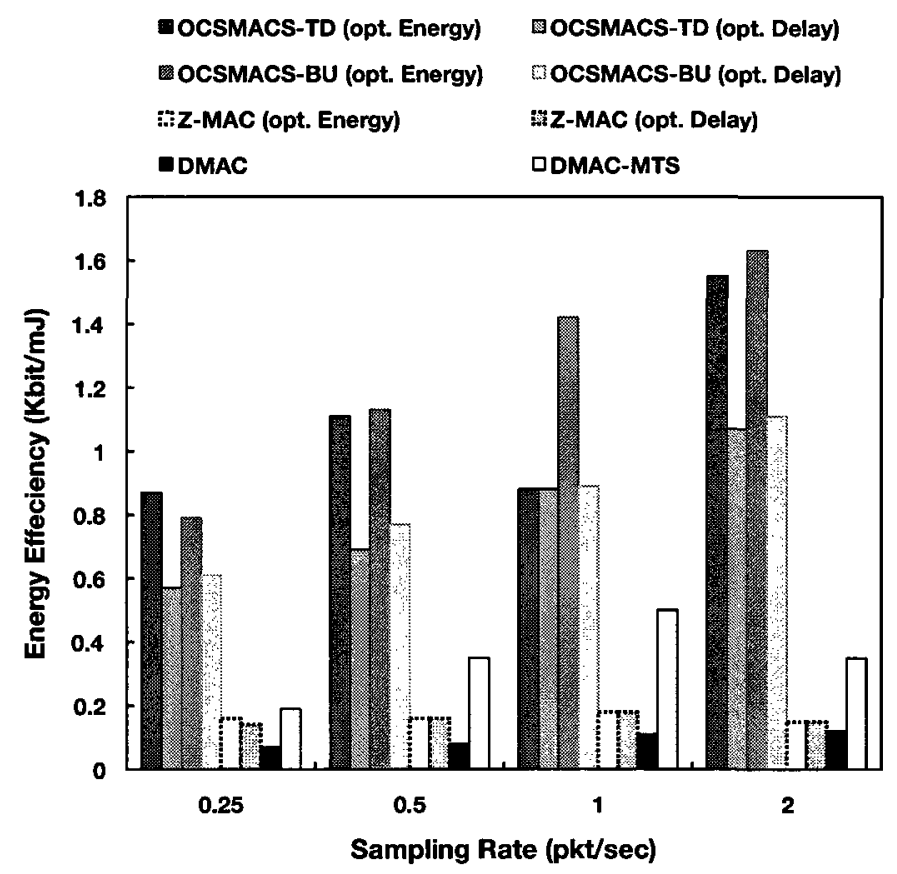

Figure 5.21: Energy efficiency of all protocols, $N=100,10$ sources. Results for both energy-efficiency and 1 s end-to-end delay applications. 


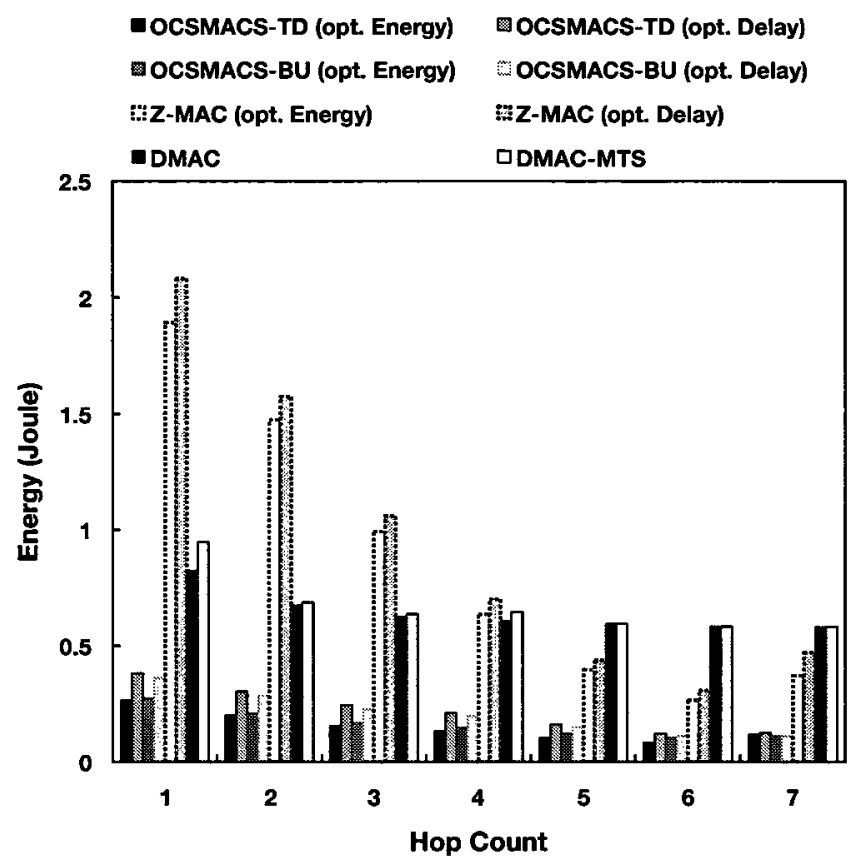

Figure 5.22: Energy consumption of nodes at different hops, $N=100,10$ sources, $r_{\text {sampling }}$ $=2 \mathrm{pkt} / \mathrm{sec}$. Results for both energy-efficiency and 1s end-to-end delay applications.

\subsection{Discussion}

Large sensor networks: Simulation results presented in this chapter up-to are for single cluster networks of up-to 160 nodes, while WSNs might consists of hundreds or even thousand of sensors. This is not a limitation of OCSMACS in handling such large number of nodes. Instead the reason is due the limitation of the simulation environment (speed and memory requirement).

Nonetheless, OCSMACS can operate in a multiclustered sensor network architecture in which a number of sinks are deployed along sensors, Figure 5.23. A distinct group of sensors associated with a sink from a cluster. Adjacent clusters are assumed to operate in different channel to limit interference. In addition, a hierarchical architecture can be used where a group of sinks for another level in the network hierarchy. 


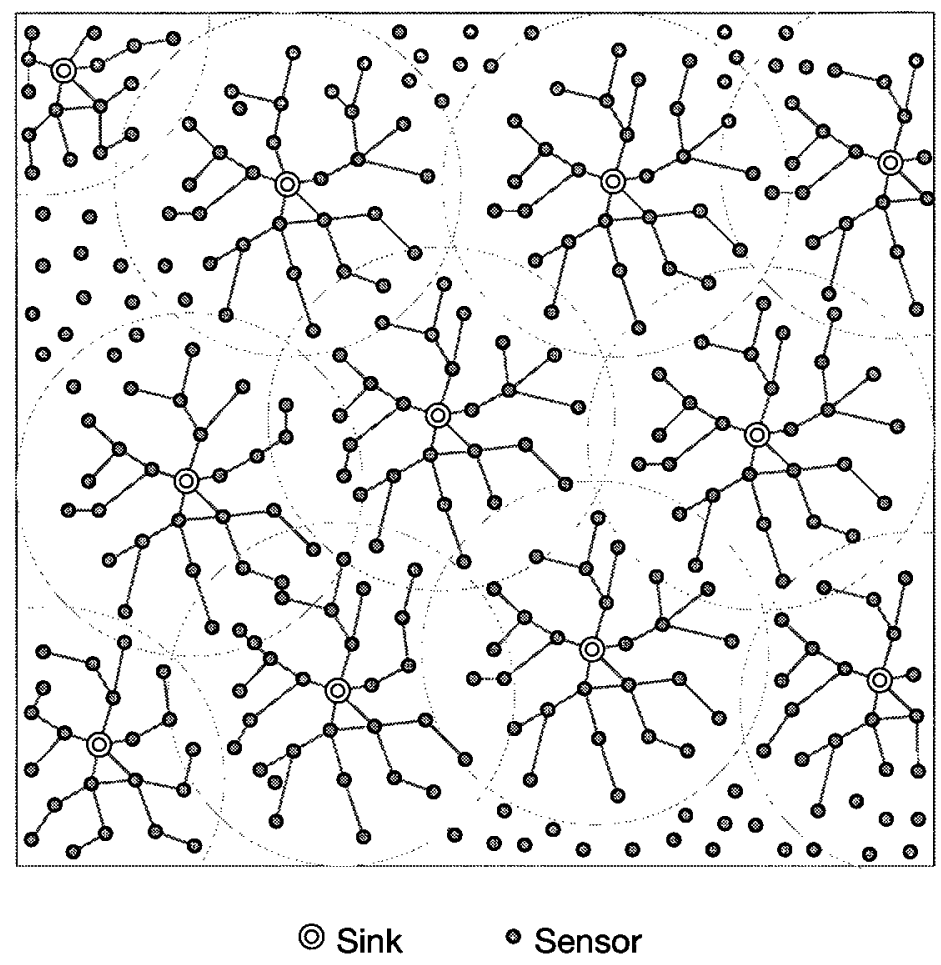

Figure 5.23: Multiclustered WSN.

There are several issues that need to be addressed in such architecture:

- Association and synchronization: When powered up, a sensor node should listen to all channels for possible Beacon. Based on the received signal strength, the node associates and synchronizes with the sink of the largest signal strength.

- Inter-cluster interference: The choice of channels among adjacent clusters affects inference experienced by nodes in the overlapping regions. To minimize this interference, adjacent clusters need to operate on channels that are further apart. In addition to this OCSMACS should have the mechanisms to handle inter-cluster interference if it exists. A possible solution is that sinks exchange 
scheduling information and resolve inter-cluster conflicts before transmitting SchDl frames. Another less desirable solution is to use unoverlapped time frames such that the end of cluster $_{i}(\operatorname{frame}(x))$ is the beginning of $\operatorname{cluster}_{j}(\operatorname{frame}(x))$ assuming cluster $_{i}$ and cluster $_{j}$ are adjacent. This solution is less desirable because it might cause large delays, specially if the frame duration is long.

- Cluster size: the dimension and the number of nodes in the cluster is affected by many factors, some of which are:

1. Transmission range of the sink.

2. Application delay, delaymax: having a large cluster means more nodes within the cluster. This would results in long TDMA frames and large SchDl frames, subsequently the end-to-end delay would increase. Therefore, the cluster size should be bounded such that the delay requirement of the application is met.

3. Scheduling overhead: the larger the cluster size the larger the ScHDL frame size, the longer ScheduleREQ slot duration - $T_{R E Q}$ and more request transmissions. The result is a increase in the scheduling energy overhead.

Fault tolerance: Another important assumption made about OCSMACS is that the sink has an access to a continues power supply and it is a fail proof node. However, in practical situations the possibility of sink failures cannot be ruled out. Fault tolerance can be achieved by deploying redundant sinks, e.g. a backup sink for an active one. Each redundant sink closely monitors current operation of the active sink in the cluster. Parameters such as synchronization periods and frame duration are used to help the backup sink to take over the cluster in case of failures. 


\subsection{Summary}

This chapter presents and discusses simulation-based evaluation of PROGRESSIVE and OCSMACS. In evaluating PROGRESSIVE, two important parameters are optimized: $B W_{N L I S T}$ and $t_{\text {discovery }}$. Simulation results show that the novel design of PROGRESSIVE makes it a time- and energy-efficient topology discovery and scheduling setup protocol in comparison to DRAND. It reduces energy consumption by a considerable factor with respect to DRAND, for a variable number of nodes. This is due to PROGRESSIVE's ability to run for a shot duration and achieve $100 \%$ scheduling efficiency. With respect to relative network density, PROGRESSIVE maintains low energy consumption whereas for DRAND energy consumption increases sharply when the relative density increases from 5 to 10 nodes.

Two different applications were used to evaluate OCSMACS: an application which requires optimal energy efficiency (delay is not important) and an application which requires the maximum delay to be 1s (energy is not a major concern). Simulations of three other protocols are also performed: S-MAC, DMAC, Z-MAC and ALWAYSON. Similar to PROGRESSIVE, OCSMACS, S-MAC and Z-MAC are all optimized to achieve the best results for the specified application.

Simulation results show that for variable network sizes, OCSMACS has a superior energy efficiency performance compared to other protocols. It consumes the least amount of energy per node, while maintaining a high throughput, hence, the highest energy efficiency. Even when optimized for a 1s delay, OCSMACS manages to remain the most energy efficient protocol. Similar performance is achieved when the sampling rate is varied in the $[0.25-2] \mathrm{pkt} / \mathrm{sec}$. 
In addition, OCSMACS shows a better performance with respect to network lifetime compared to Z-MAC and S-MAC. Specifically, when the network consists of 40 nodes, OCSMACS extends network lifetime by 3.5 years compared to Z-MAC and S-MAC. On the other hand, when the network is 160 nodes, OCSMACS extends lifetime by about one year. 


\section{Chapter 6}

\section{Correlation-aware OCSMAC}

\subsection{Motivation}

Sensor networks generate data of a certain physical phenomena, which by nature, are spatially and temporally correlated [111]. The spatial correlation results in redundancy in the data transmitted back to the sink. This gives network designers a room to improve energy efficiency by incorporating Data Aggregation, or also called Data Fusion, techniques that compress data propagating through active nodes (thus reducing redundancy) before further transmission towards the Sink.

Top-Down and Bottom-Up scheduling schemes have been proposed to facilitate the flow of data from nodes to the sink in a convergecast application. The default assumption is that each node is allocated SEND slots to transmit whatever it receives. Therefore, the number of SEND slots assigned to a node is at least the same as the number of assigned RECEIVE slots. In addition, both scheduling schemes do not guarantee that a node in the $i^{\text {th }}$ hop transmits its own data after it receives data from all neighbors in the $\left(i^{t h}+1\right)$ hop. These two characteristics of Top-Down and Bottom-Up 
scheduling might not be effective when data aggregation is to be carried out by nodes since:

- Equating the number of SEND slots to the number of RECEIVE ones may result in slots not being used. For example, Figure 3.12(b), if data is $100 \%$ correlated and aggregation is taking place in node 5, then a packet received from node 8 can be combined with a packet originating from node 3 in a single packet and transmitted in one slot. Instead Bottom-Up scheduling assigns two slots to node 5. This could lead to a waste of network and energy resources as well as undesirable delays.

- Ordering of SEND slots can render data aggregation useless especially in the case of Top-Down scheduling. This can be observed in Figure 3.12(a) where node 1 and node 7 have been assigned SEND in slot zero. This means that both nodes are likely to transmit their data before receiving from their child nodes. If data is correlated, then, only a few are aggregated. This problem is less severe in Bottom-Up scheduling.

Therefore, the objective of this chapter is to propose an enhanced scheduling schemes that are well suited for correlated data gathering applications. Section 6.2 gives a brief overview of past research in the area of data correlation aware energy efficient protocols for wireless sensor networks. Section 6.3 presents two correlation models that mimic some of those applications. Section 6.4 discusses the proposed Correlation-aware scheduling scheme, then Section 6.5 shows how the this scheduling scheme is integrated with OCSMACS. Then, an analytical model is given in Section 6.6 to demonstrate the effectiveness of the new scheme. Finally, through simulation, 
Section 6.7 evaluates the proposed scheduling scheme when combined with OCSMACS and compares it with both Top-own and Bottom-Up scheduling.

\subsection{Related Work}

Data aggregation has been widely investigated technique used in prolonging sensor network lifetime. Previous research has primarily been focusing on aggregation-driven routing schemes. For example, [111] study the performance of three different routing with compression schemes, i.e. Distributed Source Coding, Routing Driven Compression and Compression Driven Routing. In addition, a near-optimal network clustering scheme which maximizes the energy efficiency is presented. Similarly, [113] utilizes a non-linear optimization problem to find the optimal clustering structure for data aggregation minimizing the total energy cost; that clustering scheme is called "Annular Slicing-based Clustering". Both [111] and [113] conclude that varying the cluster size, an optimal clustering which minimizes energy consumption for a wide range of spatial data correlation is achieved.

Recent work in [114] aims at maximizing network lifetime by jointly optimizing routing and data aggregation. An approximate smoothing function which exploits the specific network structure is derived. On the other hand, [115] identify an optimal energy efficient routing problem that in addition to transmission cost it considers the aggregation cost. The reason is that the aggregation cost may vary due to variable data characteristics or encryption/decryption overhead. In addition a Minimum Fusion Steiner Tree that can guarantee a specific approximation ratio to the optimal solution is presented. Despite the energy gain achieved by aggregation-driven routing optimization, the advantage it offers is limited if not combined with a MAC protocol 
which utilizes the proposed enhanced routing and clustering algorithms.

Little work has been done on integrating data aggregation with the design of MAC protocols. Two examples of such efforts are LEACH [51] and PEGASIS [116] . As discussed in Section 2.3.3, one of the main assumptions made about LEACH is that data generated by neighboring nodes is highly correlated hence, the cluster-head is able to aggregate in a single packet all data received from nodes in the cluster before it is sent to the BS. In PEGASIS, sensors form chains along which a node transmits and receives from a nearby neighbor. Data aggregation is then performed while data moves from node to node. Similar to LEACH, it is assumed that data from different nodes can be fused and compressed generating a single data packet.

Despite the performance gain achieved by both LEACH and PEGASIS, making a generalizing assumption that there is a continuos stream of data might not be applicable in all situations. Both protocols do not define a sleep schedule, instead they mainly define the mechanisms through which nodes know where and when data should be transmitted. In addition, LEACH assumes CDMA capable nodes and their transmit power can be high enough to reach the BS in one hop. The other limitation of LEACH and PEGASIS is that they do not provide an evaluation to the cost of performing data aggregation or the effect of having a varying correlation in the transmitted data. 


\subsection{Correlation Models}

\subsubsection{Global Correlation Model}

The Global Correlation (GC) model, presented in [113], describes the joint entropy; a measure of the amount of information generated by a collection of sources [118]. A correlation parameter, $\rho \in[0,1]$ is used to characterize the extent of spatial and temporal correlation of the data. The value of $\rho$ is set on the basis of the environment conditions during the sensing and the nature of the data. For a certain query response or event type this value is assumed to be known; it is constant during data generation and collection time. The model also defines $H_{0}$ as the entropy of any single source node with a "correlated" part with entropy $\rho H_{0}$ and an "uncorrelated part" with entropy $(1-\rho) H_{0}$. The joint entropy of any two sources is obtained from the following two parts:

1. uncorrelated part $=2(1-\rho) H_{0}$

2. correlated part $=\rho H_{0}$

The joint entropy is:

$$
\text { Joint entropy }(G C)=H_{0}+(1-\rho) H_{0}
$$

Based on this model, data from different sources is opportunistically aggregated. Whenever correlated data happens to meet at a receiving node during a receive slot that node aggregates it with its own data, if any is queued, and then transmits it on the upcoming send slot. 


\subsubsection{Sensing Range Correlation Model}

The global correlation model assumes that the level of correlation between any two sources is the same for all nodes in a network. This model might not be applicable when the network is sparse and the average distance between some neighboring nodes exceeds the sensing range. For example, the IMB400 [119] is an Imote2 [120] sensor board equipped with a motion detection sensor with $5 m$ maximum range but the Imote 2 itself can transmit at a maximum range of $30 \mathrm{~m}$. Figure 6.1 shows an example of two nodes that have no overlapped sensing area. Data generated by node A (monitoring a target) might not be correlated with that generated by node B, i.e. $\rho$ is zero.

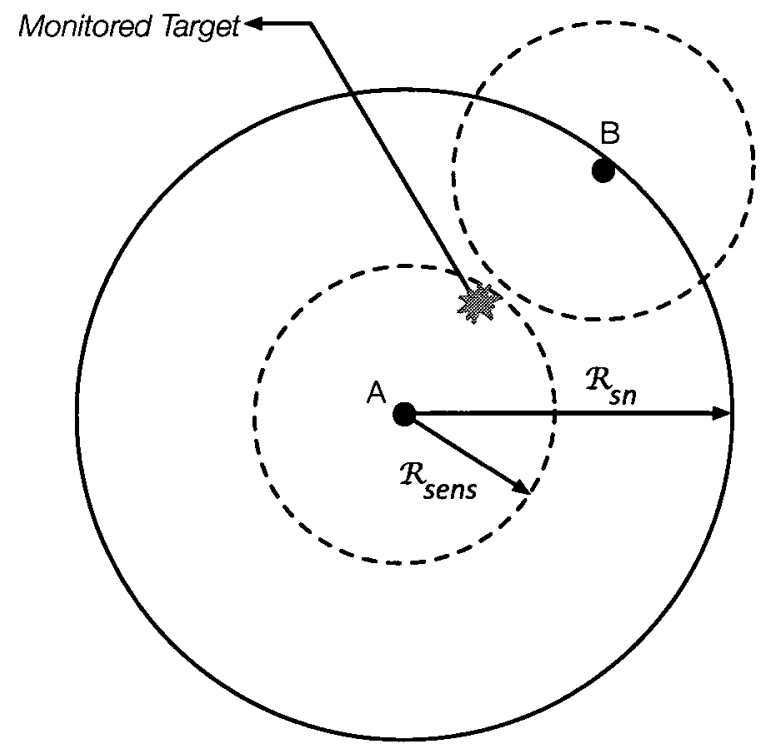

Figure 6.1: Nodes A and B are within each other's transmission range, but their data are not necessarily correlated because the monitored target is outside B's coverage area. 
The Sensing Range Correlation (SRC) model redefines the joint entropy of any two sources as:

$$
\text { Joint entropy }(S R C)= \begin{cases}H_{0}+\left(1-\rho_{s_{0} s_{1}}\right) H_{0} & d\left(s_{0}, s_{1}\right) \leq 2 \mathcal{R}_{\text {sense }} \\ 2 H_{0} & d\left(s_{0}, s_{1}\right)>2 \mathcal{R}_{\text {sense }}\end{cases}
$$

The uncorrelated and the correlated parts are:

$$
\begin{gathered}
\text { Uncorrelated part }= \begin{cases}2\left(1-\rho_{s_{0} s_{1}}\right) H_{0} & d\left(s_{0}, s_{1}\right) \leq 2 \mathcal{R}_{\text {sense }} \\
2 H_{0} & d\left(s_{0}, s_{1}\right)>2 \mathcal{R}_{\text {sense }}\end{cases} \\
\text { Correlated part }= \begin{cases}\rho_{s_{0} s_{1}} H_{0} & d\left(s_{0}, s_{1}\right) \leq 2 \mathcal{R}_{\text {sense }} \\
0 & d\left(s_{0}, s_{1}\right)>2 \mathcal{R}_{\text {sense }}\end{cases}
\end{gathered}
$$

where $d\left(s_{0}, s_{1}\right)$ is the distance between the two sources; $s_{0}$ and $s_{1}, \mathcal{R}_{\text {sense }}$ is the sensing range and $\rho_{s_{0} s_{1}}$ is the correlation parameter specific to each pair $\left(s_{0}, s_{1}\right)$.

The disadvantages of the sensing range correlation model are that it requires nodes to have their location information and to communicate this information in the transmitted data packets. Obtaining location information and the communication overhead associated with that might exceed cost-wise the savings achieved by aggregation. Exploring localization models and/or other correlation models and their implementations is beyond the scope of this dissertation. 


\subsection{Correlation-aware Scheduling}

Spatial-temporal scheduling assumes that each node that receives a data packet needs to forward it as it is. This means that during the data collection period the number of assigned SEND slots is at least equal to the number of RECEIVE slots. If data is $100 \%$ correlated, then the nodes would be assigned considerably higher number of slots than needed. Forcing nodes to wake-up at specific time slots without transmitting or receiving data is a waste of energy and network resources.

Therefore, Correlation-Aware (CA) scheduling allocates enough slots based on the level of correlation in the collected data. In addition, it is similar to Bottom-Up scheduling with the difference that a node $n_{i}$ in Level $_{i}$ can only be assigned SEND at a specific time slot if $n_{i} \in \mathcal{L}$ where $\mathcal{L}$ is the list of all leaf nodes (a leaf node in Level $_{i}$ is any node whose neighbors in Level $_{i+1}$, have all been assigned SEND in previous cycles).

The number of SEND slots - $\mathcal{T}_{S E N D}$ assigned to a node is:

$$
\mathcal{T}_{S E N D}=\left\lceil\frac{\mathcal{D}_{\text {sn }}+(1-\rho) \mathcal{D}_{\text {neighbors }}+o}{P K T_{\text {length }}}\right\rceil
$$

where $\mathcal{D}_{s n}$ is the total data bytes generated by a node, $\mathcal{D}_{\text {neighbors }}$ is the total data bytes received from neighboring child nodes, $P K T_{\text {length }}$ is the length of a single data packet in bytes and $o$ is the overhead associated with packets' header. 


\subsection{OCSMACS with Correlation-aware Schedul- ing}

OCSMACS integrated with the Global Correlation-aware scheduling is called OCSMACSGCA; OCSMACS integrated with the Sensing Range Correlation-aware scheduling is called OCSMACS-SRCA. OCSMACS-TD and OCSMACS-BU can also opportunistically aggregate data so that when packets are received, nodes check for queued packets to be aggregated with the newly received ones. If so, aggregation takes place before data transmission and if not, the received packets are transmitted without. OCSMACS-TD and OCSMACS-BU with opportunistic aggregation are denoted by OCSMACS-TD+ and OCSMACS-BU+, respectively.

The duration of a slot must be adjusted to account for the aggregation time. This gives each node the time to aggregate the received packets before the upcoming SEND slot. Therefore, the slot duration is rewritten as:

$$
T_{\text {slot }}^{\text {OCSMACS }}{ }^{+}=P K T_{\text {length }} \times T_{\text {Byte }}+T_{\text {aggregation }}
$$

\subsection{Analytical Models}

Correlation-aware scheduling, in general, is a leafnode-based scheduling scheme. However, the number of SEND slots assigned to leafnodes in a particular level Level $_{i}$ depends on the number of RECEIVE slots already assigned as well as on the correlation parameter $(\rho)$ (equation (6.3)). In this section, expressions for the total number of slots and the time required to collect a single data packet from each sensor in a network are given. In addition, the communication energy consumption and the 
achievable energy gain resulting from the aggregation is provided.

\subsubsection{Scheduling Delay}

To find the required $\tau$ to collect $N$ aggregated data packets from $N$ nodes using global Correlation-aware and sensing range-aware scheduling schemes, the following definition and assumptions are made:

- The network is divided into hypothetical levels (regions) based on hop count so that Level $_{i}$ is $i+1$ hops from the sink. The total number of levels is $k$.

- $N$ is the total number of nodes in the network; there are $n_{i}$ nodes in Level $_{i}$ of the network.

- The time is divided into equivalent time slots. Each time slot $(\tau)$ is long enough for a single packet transmission. The same slot can be assigned to different nonconflicting nodes (or links).

- $\mathcal{D}_{i}(\tau)$ is the number of data packets in Level $_{i}$ at the beginning of slot $\tau$. Therefore, for each $\operatorname{Level}_{i}, \mathcal{D}_{i}(0)$ is $n_{i}$.

- Let $q_{i}$ denote the proportion of all Level $_{i}$ 's nodes which can transmit simultaneously without conflicts during slot $\tau$.

- Let $q_{i}^{l}$ denote the proportion of Level ${ }_{i}$ 's Leafnodes which have data in their queues and can transmit simultaneously without conflicts during slot $\tau$.

- Let $p_{i}$ denote the proportion of Level ${ }_{i}$ 's Leafnodes which have data in their queues and can transmit simultaneously to neighbors within a correlated sensing range without conflict during slot $\tau$. Therefore, $p_{i} \subset q_{i}^{l}$. 
- A single data packet is generated from each node in the network. Therefore, the total number of packets to be collected prior to aggregation is $N$.

\section{Global Correlation-aware Scheduling}

To find the minimum $\tau$ to collect $n$ data packets from $N$ nodes using global correlationaware scheduling, $\mathcal{D}_{i}(\tau)$ for $\tau>0$ is computed:

$$
\begin{gathered}
\mathcal{D}_{i}(\tau)=\mathcal{D}_{i}(\tau-1)+q_{i+1}^{l}(1-\rho) \mathcal{D}_{i+1}(\tau-1)-q_{i}^{l} \mathcal{D}_{i}(\tau-1) \\
=\left(1-q_{i}^{l}\right) \mathcal{D}_{i}(\tau-1)+q_{i+1}^{l}(1-\rho) \mathcal{D}_{i+1}(\tau-1) \\
\mathcal{D}_{i}(\tau)= \begin{cases}\left(1-q_{i}^{l}\right) \mathcal{D}_{i}(\tau-1)+q_{i+1}^{l}(1-\rho) \mathcal{D}_{i+1}(\tau-1) & 0 \leq i<k-1 \\
\left(1-q_{i}^{l}\right) \mathcal{D}_{i}(\tau-1) & i=k-1\end{cases}
\end{gathered}
$$

\section{Sensing Range Correlation-aware Scheduling}

The same approach as the one discussed above is used to find the time required to collect packets from all nodes using sensing range correlation-aware scheduling. However, in the sensing range correlation model not all data received from Leafnodes is subject to aggregation. Instead, only data received from Leafnodes that are within the correlated sensing range of the receiving nodes are aggregated. Other data will be forwarded with no aggregation. 
Therefore, for $\tau>0, \mathcal{D}_{i}(\tau)$ is given by:

$$
\begin{gathered}
\mathcal{D}_{i}(\tau)=\mathcal{D}_{i}(\tau-1)+p_{i+1} q_{i+1}^{l}\left(1-\rho_{s_{0} s_{1}}\right) \mathcal{D}_{i+1}(\tau-1) \\
\quad+\left(1-p_{i+1}\right) q_{i+1}^{l} \mathcal{D}_{i+1}(\tau-1)-q_{i}^{l} \mathcal{D}_{i}(\tau-1) \\
=\left(1-q_{i}^{l}\right) \mathcal{D}_{i}(\tau-1)+q_{i+1}^{l}\left(1-p_{i+1} \rho_{s_{0} s_{1}}\right) \mathcal{D}_{i+1}(\tau-1) \\
\mathcal{D}_{i}(\tau)= \begin{cases}\left(1-q_{i}^{l}\right) \mathcal{D}_{i}(\tau-1)+q_{i+1}^{l}\left(1-p_{i+1} \rho_{s_{0} s_{1}}\right) \mathcal{D}_{i+1}(\tau-1) & 0 \leq i<k-1 \\
\left(1-q_{i}^{l}\right) \mathcal{D}_{i}(\tau-1) & i=k-1\end{cases}
\end{gathered}
$$

The delay for both correlation-aware scheduling schemes $T_{\text {multihop }}^{C A}$ is:

$$
T_{\text {multihop }}^{C A}=\min (\tau) \times T_{\text {slot }}^{O C S M A C S^{+}} \quad \text { s.t. } \quad \sum_{i=0}^{L-1} \mathcal{D}_{i}(\tau)=0
$$

From $\mathcal{D}_{i}(\tau)$ and $T_{\text {multihop }}^{C A}$ it can be observed that:

1. $q_{i}^{l} \leq q_{i}$. Thus, more slots are needed to transmit all data packets in Level $_{i}$.

2. $T_{\text {slot }}^{O C S M A C S^{+}}=T_{\text {slot }}^{O C S M A C S}+T_{\text {aggregation. }}$ Therefore, $T_{\text {multihop }}^{C A}$ is worse than $T_{\text {multihop }}^{B U}$ and $T_{\text {multihop }}^{T D}$ by at least $\tau \times T_{\text {aggregation }}$.

Therefore, correlation-aware scheduling tends to increase delay compared to BottomUp and Top-Down scheduling especially when $\rho$ is small (in the case of the Global Correlation model) or the sensing range is considerably smaller than the transmission range (in the case of the Sensing Range Correlation model). 


\subsubsection{Energy Consumption}

The primary objective of data aggregation is to reduce energy consumption by suppressing redundant data while forwarding packets to the sink. The overall energy reduction results mainly because of the reduction in consumed communication energy. On the other hand, data aggregation and fusion are performed in-network and this would cause more processing load on the mico-controller. As a result, reducing communication energy is reflected as an increase in the processing energy. In this part, only the micro-controller energy and transmit and receive energy for the global correlation-aware and sensing range-aware scheduling schemes is computed. The same network model as in Section 4.3 is used.

1. All nodes draw the same current levels when communicating data or when carrying out internal processing tasks.

2. All nodes have the same sampling rate $\left(r_{\text {sampling }}\right)$ and the same packet length $P K T_{\text {length }}$ (in bytes).

3. Packets transmitted by nodes in ring $_{i}$ must be relayed by nodes in $r i n g_{i-1}$ where $0<i<k$.

4. Let $\lambda_{i}$ be the total number of packets generated (sampled) by nodes in ring . $_{\text {. }}$

\section{Micro-controller Energy}

The mico-controller active energy is consumed while conducting data aggregation plus other operations. It can be given by:

$$
E_{M C U}^{G C A}=T_{M C U}^{G C A} \times \mathcal{P}_{M C U}^{a c t i v e}
$$


where $T_{M C U}^{G C A}$ is the total time during which the MCU is $\mathrm{ON}$ during communication and data processing. $\mathcal{P}_{M C U}^{\text {active }}$ is the MCU power consumption during the active mode. In-network data aggregation would increase the MCU active time due to additional data processing. On the contrary, the reduction in communication would reduce it. Therefore, we can write $T_{M C U}^{G C A}$ as:

$$
\begin{aligned}
T_{M C U}^{G C A} & =T_{M C U}^{a c t i v e}+T_{M C U}^{\text {aggregation }} \\
& =T_{T X}^{G C A}+T_{R X}^{G C A}+T_{L I S T E N}^{G C A}+T_{M C U}^{\text {aggregation }}
\end{aligned}
$$

where $T_{M C U}^{\text {aggregation }}$ is the time during which the micro-controller is performing data aggregation. Assuming that $\mathcal{P}_{M C U}^{G C A}$ and $\mathcal{P}_{M C U}^{a c t i v e}$ are the same, $E_{M C U}^{G C A}$ can be written in terms of $E_{M C U}^{a c t i v e}$ (in Equation(4.23)), as:

$$
E_{M C U}^{G C A}=\left[T_{T X}^{G C A}+T_{R X}^{G C A}+T_{L I S T E N}^{G C A}+T_{M C U}^{\text {aggregation }}\right] \times \mathcal{P}_{M C U} .
$$

\section{Global Correlation-aware Scheduling Transmit Energy}

The transmit energy is related to the number of total data transmitted at each ring as previously shown in equation (4.7). In the case of the Global Correlation model, data received from ring $_{i+1}$ is aggregated with data at $r i n g_{i}$ based on the correlation parameter $\rho$.

Therefore, the total number of aggregated packets transmitted by $\operatorname{ring}_{i}, \lambda_{i}^{G C A^{t x}}$ is:

$$
\lambda_{i}^{G C A^{t x}}=\lambda_{i+1}^{G C A^{t x}}+\lambda_{i}-\rho \lambda_{i+1} .
$$


Over a time period $t, \lambda_{i}^{G C A^{t x}}$ is given as:

$$
\lambda_{i}^{G C A^{t x}}=\lambda_{i+1}^{G C A^{t x}}+\left(t \times r_{\text {sampling }} \times \delta\right)\left(A_{i}-(1-\rho) A_{i+1}\right) .
$$

The total transmission energy $E^{G C A^{t x}}$ can be expressed as the sum of transmission energies of all rings:

$$
\begin{aligned}
E^{G C A^{t x}} & =\sum_{i=0}^{k-1} E_{i}^{G C A^{t x}} \\
& =\sum_{i=0}^{k-1} \lambda_{i}^{G C A^{t x}} \times P K T_{\text {length }} \times T_{B y t e} \times \mathcal{P}_{t x}
\end{aligned}
$$

\section{Global Correlation-aware Scheduling Receive Energy}

The reception energy is consumed while receiving the aggregated packets transmitted by nodes in other rings. The total number of aggregated packets received by nodes in $\operatorname{ring}_{i}$ during a period of time $t, \lambda_{i}^{r x}$ is:

$$
\lambda_{i}^{G C A^{r x}}=\lambda_{i+1}^{G C A^{t x}} .
$$

The total receive energy $E_{r x}^{G C A}$ is:

$$
\begin{aligned}
E^{G C A^{r x}} & =\sum_{i=0}^{k-1} E_{i}^{G C A^{r x}} \\
& =\sum_{i=0}^{k-1} \lambda_{i}^{G C A^{r x}} \times P K T_{\text {length }} \times T_{\text {Byte }} \times \mathcal{P}_{r x}
\end{aligned}
$$




\section{Global Correlation-aware Scheduling Energy Gain}

The energy gain is the percentage of energy reduction due to aggregation. It can be expressed as:

$$
\mathcal{G}^{G C A}=1-\frac{E_{t x}^{G C A}+E_{r x}^{G C A}+E_{M C U}^{G C A}}{E_{t x}+E_{r x}+E_{l i s t e n}+E_{M C U}^{a c t i v e}} .
$$

Substitute equations (4.7), (4.11), (4.17), (6.12), (6.5) and (6.14) in equation (6.15). For the sake of simplicity, let's assume that $\forall i, \lambda_{i}=1$ and $T_{P K T}$ is the packet transmission time. Then $\mathcal{G}^{G C A}$ can be expressed as:

$$
\begin{gathered}
\mathcal{G}^{G C A}=1-\frac{T_{P K T} \sum_{i=0}^{k-1}\left[\mathcal{P}_{t x} \lambda_{i}^{G C A^{t x}}+\mathcal{P}_{r x} \lambda_{i}^{G C A^{r x}}\right]+E_{M C U}^{G C A}}{T_{P K T} \sum_{i=0}^{k-1}\left[\mathcal{P}_{t x} \lambda_{i}^{t x}+\mathcal{P}_{r x} \lambda_{i}^{r x}\right]+E_{l i s t e n}+E_{M C U}^{a c t i v e}} \\
=1-\frac{T_{P K T} \sum_{i=0}^{k-1}\left[\mathcal{P}_{t x}\left(\lambda_{i+1}^{G C A^{t x}}+\lambda_{i}-\rho \lambda_{i+1}\right)+\mathcal{P}_{r x} \lambda_{i+1}^{G C A^{t x}}\right]+E_{M C U}^{G C A}}{T_{P K T} \sum_{i=0}^{k-1}\left[\mathcal{P}_{t x}\left(\lambda_{i}+\lambda_{i+1}^{t x}\right)+\mathcal{P}_{r x} \lambda_{i+1}^{t x}\right]+E_{l i s t e n}+E_{M C U}^{a c t i v e}}
\end{gathered}
$$

As expected, when $\rho$ is $0, \lambda_{i+1}^{G C A^{t x}}=\lambda_{i+1}^{t x}$ and $E_{M C U}^{G C A}=E_{M C U}^{\text {active }}\left(E_{M C U}^{\text {aggregation }}=0\right)$. Therefore, gain is not achieved $\left(\mathcal{G}^{G C A}=0\right)$. On the other hand, when $\rho$ is greater than 0 , a positive gain is only achievable if:

$$
\begin{aligned}
E_{M C U}^{\text {aggregation }}< & T_{P K T}\left[\left(\mathcal{P}_{t x}+\mathcal{P}_{M C U}^{\text {active }}\right) \sum_{i=0}^{k-1}\left(\lambda_{i}^{t x}-\lambda_{i}^{G C A^{t x}}\right)\right] \\
& +T_{P K T}\left[\left(\mathcal{P}_{r x}+\mathcal{P}_{M C U}^{a c t i v e}\right) \sum_{i=0}^{k-1}\left(\lambda_{i}^{r x}-\lambda_{i}^{G C A^{r x}}\right)\right] \\
& +\left(\mathcal{P}_{\text {listen }}+\mathcal{P}_{M C U}^{\text {active }}\right) \times T_{\text {listen }} .
\end{aligned}
$$




$$
\begin{aligned}
E_{M C U}^{\text {aggregation }}< & T_{P K T}\left[\left(\mathcal{P}_{t x}+\mathcal{P}_{M C U}^{\text {active }}\right) \sum_{i=0}^{k-1}\left(\lambda_{i+1}^{t x}+\rho \lambda_{i+1}-\lambda_{i+1}^{G C A^{t x}}\right)\right] \\
& +T_{P K T}\left[\left(\mathcal{P}_{r x}+\mathcal{P}_{M C U}^{\text {active }}\right) \sum_{i=0}^{k-1}\left(\lambda_{i+1}^{t x}-\lambda_{i+1}^{G C A^{t x}}\right)\right] \\
& +\left(\mathcal{P}_{\text {listen }}+\mathcal{P}_{M C U}^{\text {active }}\right)\left(\frac{\rho \mathcal{D}_{\text {neighbors }}-o}{P K T_{\text {length }}} \times T_{\text {sample }}\right)
\end{aligned}
$$

This inequality assumes that $T_{M C U}^{\text {aggregation }}$ is the same for all values of $\rho$. However, if there are applications in which the processing time increases as the level of aggregation increases, the inequality must be modified to account for variations in $\rho$. To ensure a positive energy gain when processing time increases as correlation increases, the following condition must be met:

$$
\begin{aligned}
E_{M C U}^{\text {aggregation }}< & \frac{T_{P K T}\left[\left(\mathcal{P}_{t x}+\mathcal{P}_{M C U}^{\text {active }}\right) \sum_{i=0}^{k-1}\left(\lambda_{i+1}^{t x}+\rho \lambda_{i+1}-\lambda_{i+1}^{G C A^{t x}}\right)\right]}{C} \\
+ & \frac{T_{P K T}\left[\left(\mathcal{P}_{r x}+\mathcal{P}_{M C U}^{\text {active }}\right) \sum_{i=0}^{k-1}\left(\lambda_{i+1}^{t x}-\lambda_{i+1}^{G C A^{t x}}\right)\right]}{C} \\
+ & \frac{\left(\mathcal{P}_{\text {listen }}+\mathcal{P}_{M C U}^{\text {active }}\right)\left(\frac{\rho \mathcal{D}_{\text {neighbors }}-o}{P K T_{\text {length }}} \times T_{\text {sample }}\right)}{C}
\end{aligned}
$$

where $C>0$ is an increasing function of $\rho$.

\section{Sensing Range Correlation-aware Scheduling Transmit Energy}

Unlike the Global Correlation-aware scheduling, in Sensing Range Correlation-aware scheduling nodes in ring $_{i}$ can only aggregate packets received from $r i n g_{i+1}$ only if the receivers are within the correlated sensing range of the transmitters. 
The total number of aggregated packets transmitted by $r i n g_{i}, \lambda_{i}^{S R C A^{t x}}$ is:

$$
\lambda_{i}^{S R C A^{t x}}=\lambda_{i+1}^{S R C A^{t x}}+\lambda_{i}-p_{i+1} \rho_{s_{0} s_{1}} \lambda_{i+1}
$$

where $s_{0} \in p_{i+1}$ and $s_{1} \in n_{i}$. When all neighboring nodes are within each other's sensing range, i.e. $p_{i}=1$, the Sensing Range Correlation-aware scheduling performs as the Global Correlation-aware scheduling so that $\lambda_{i}^{S R C A^{t x}}$ becomes:

$$
\lambda_{i, p_{i}=1}^{S R C A^{t x}}=\lambda_{i+1}^{S R C A^{t x}}+\lambda_{i}-\rho \lambda_{i+1}
$$

The total transmission energy $E^{S R C A^{t x}}$ is given by:

$$
\begin{aligned}
E^{S R C A^{t x}} & =\sum_{i=0}^{k-1} E_{i}^{S R C A} \\
& =\sum_{i=0}^{k-1} \lambda_{i}^{S R C A^{t x}} \times P K T_{\text {length }} \times T_{\text {Byte }} \times \mathcal{P}_{t x}
\end{aligned}
$$

\section{Sensing Range Correlation-aware Scheduling Receive Energy}

The total number of aggregated packets received by nodes in $r i n g_{i}$ during a period of time $t, \lambda_{i}^{S R C A^{r x}}$ takes the same form as $\lambda_{i}^{G C A^{r x}}$ :

$$
\lambda_{i}^{S R C A^{r x}}=\lambda_{i}^{S R C A^{t x}}, \quad 0<i<k-1
$$


The total receive energy $E^{G S R C A^{r x}}$ is:

$$
\begin{aligned}
E^{S R C A^{r x}} & =\sum_{i=0}^{k-1} E_{i}^{S R C A^{r x}} \\
& =\sum_{i=0}^{k-1} \lambda_{i}^{S R C A^{r x}} \times P K T_{\text {length }} \times T_{\text {Byte }} \times \mathcal{P}_{r x}
\end{aligned}
$$

\section{Sensing Range Correlation-aware Scheduling Energy Gain}

The energy gain achieved by the Sensing Range Correlation-aware Scheduling $\mathcal{G}^{S R C A}$ is:

$$
\mathcal{G}^{S R C A}=1-\frac{T_{P K T} \sum_{i=0}^{k-1}\left[\mathcal{P}_{t x}\left(\lambda_{i+1}^{S R C A^{t x}}+\lambda_{i}-p_{i+1} \rho_{s_{0} s_{1}} \lambda_{i+1}\right)+\mathcal{P}_{r x} \lambda_{i+1}^{S R C A^{t x}}\right]+E_{M C U}^{S R C A}}{T_{P K T} \sum_{i=0}^{k-1}\left[\mathcal{P}_{t x}\left(\lambda_{i}+\lambda_{i+1}^{t x}\right)+\mathcal{P}_{r x} \lambda_{i+1}^{t x}\right]+E_{\text {listen }}+E_{M C U}^{a c t i v e}} .
$$

When $p_{i}=1$, the Sensing Range Correlation-aware scheduling behaves as the Global Correlation-aware scheduling and both schemes have the same gain as:

$$
\mathcal{G}_{p_{i}=1}^{S R C A}=\mathcal{G}^{G C A}
$$

Similarly, SRCA scheduling can reduce the overall energy only if:

$$
\begin{aligned}
E_{M C U}^{\text {aggregation }}< & T_{P K T}\left[\left(\mathcal{P}_{t x}+\mathcal{P}_{M C U}^{a c t i v e}\right) \sum_{i=0}^{k-1}\left(\lambda_{i+1}^{t x}+p_{i+1} \rho_{s_{0} s_{1}} \lambda_{i+1}-\lambda_{i+1}^{S R C A^{t x}}\right)\right] \\
& +T_{P K T}\left[\left(\mathcal{P}_{r x}+\mathcal{P}_{M C U}^{\text {active }}\right) \sum_{i=0}^{k-1}\left(\lambda_{i+1}^{t x}-\lambda_{i+1}^{S R C A^{t x}}\right)\right] \\
& +\left(\mathcal{P}_{\text {listen }}+\mathcal{P}_{M C U}^{\text {active }}\right)\left(\frac{\rho_{s_{0} s_{1}} \mathcal{D}_{\text {neighbors }}-o}{P K T_{\text {length }}} \times T_{\text {sample }}\right)
\end{aligned}
$$


In order to achieve a positive energy gain under the condition that the processing power increases as the level of correlation increases, the following condition must be met:

$$
\begin{aligned}
E_{M C U}^{\text {aggregation }}< & \frac{T_{P K T}\left[\left(\mathcal{P}_{t x}+\mathcal{P}_{M C U}^{a c t i v e}\right) \sum_{i=0}^{k-1}\left(\lambda_{i+1}^{t x}+p_{i+1} \rho_{s_{0} s_{1}} \lambda_{i+1}-\lambda_{i+1}^{S R C A^{t x}}\right)\right]}{C} \\
+ & \frac{T_{P K T}\left[\left(\mathcal{P}_{r x}+\mathcal{P}_{M C U}^{a c t i v e}\right) \sum_{i=0}^{k-1}\left(\lambda_{i+1}^{t x}-\lambda_{i+1}^{S R C A^{t x}}\right)\right]}{C} \\
+ & \frac{\left(\mathcal{P}_{\text {listen }}+\mathcal{P}_{M C U}^{\text {active }}\right)\left(\frac{\rho_{s_{0} s_{1}} \mathcal{D}_{\text {neighbors }}-o}{P K T_{\text {length }}} \times T_{\text {sample }}\right)}{C} .
\end{aligned}
$$

\subsection{Simulation Evaluation}

In this section, the effectiveness of the proposed correlation-aware scheduling schemes when integrated with OCSMACS is demonstrated. The main objective is to establish the energy reduction gain resulting from data aggregation. $n s-2$ simulations are carried out in two parts:

- PartI: Study the performance of OCSMACS-GCA and OCSMACS-SRCA and compare them against OCSMACS-TD+ and OCSMACS-BU+.

- PartII: Study aggregation feasibility which is the feasible extent of energy savings of the proposed correlation-aware scheduling schemes under variable processing time and energy. 
In evaluating the proposed scheduling schemes, the following performance metrics are considered:

1. Aggregation Efficiency is the percentage of the total data (bits) suppressed by the aggregation while being forwarded towards the sink.

2. Energy is the total average energy consumed during the simulation time.

3. Energy Efficiency: Unlike the energy efficiency defined in Chapter 5, the energy efficiency in the context of data aggregation is the total data (bits) received plus the total data suppressed over the total average energy consumed by a sensor. In other words, it is the total energy consumed by a sensor to deliver $x$ bits of aggregated data.

4. Delay is to end-to-end delay which each packet experiences from its initial transmission until it reached the sink. In this context, each packet is a packet carrying data originating from a particular source or a packet carrying an aggregated data of many different sources.

Introducing aggregation power consumption, or energy cost, in the simulation is done by adding an artifical processing time, $T_{\text {aggregation }}$, to each slot. In such case the total processing time of each sensor is the total number of assigned RECEIVE slots times $T_{\text {aggregation. }}$ The reason for this is that $n s-2$ does not have a model for an actual sensor platform or operating system, such as TinyOS in MICA2 [23]. In real implementation, values of $T_{\text {aggregation }}$ vary according to the specific aggregation/compression algorithm, sensor platform and operating system. Nevertheless, the suggested values of $T_{\text {aggregation }}$ falls within the range of compression times determined using a TI MSP430x1611 micro-controller $[122,123]$ which is comparable to MICA2 sensors. 


\subsubsection{OCSMACS-GCA}

In this part, a network of 100 nodes and varying value of $\rho$ (from 0.1 to 1 ) is simulated. All nodes are considered to be active sources; the sampling rate is $0.1 \mathrm{pkt} / \mathrm{sec}$; and the aggregation processing time $T_{\text {aggregation }}$ is $5 \mathrm{~ms}$. The rest of the simulation parameters are the same as in Table 5.1.

Figure 6.2 shows the delay, aggregation efficiency, energy and energy efficiency of OCSMAC-GCA, OCSMACS-TD+ and OCSMACS-BU+. The general trend of all protocols is that both delay and energy consumption decreases as data becomes highly correlated. This results from the decrease in the number of slots required for aggregated data transmission (see Table 6.1). Compared to OCSMACS-BU+ and OCSMACS-TD+, OCSMACS-GCA increases the end-to-end delay by $7 \%$ and $18 \%$, respectively, when $\rho$ is less than 0.4 . This is caused by the fact that the Correlationaware scheduling postpones SEND slots' assignment until nodes receive data from all of their children. At low correlation levels delayed scheduling has a negative effect on delay. However, beyond 0.5 correlation, OCSMACS-GCA's delay continues to drop until it is the same as that of OCSMACS-TD+ when $\rho$ is one. This is a reduction of $9 \%$ compared to OCSMACS-BU+.

Delayed scheduling allows nodes to aggregate more data compared to Top-Down or Bottom-Up scheduling. Figure 6.2(b) shows that when $\rho$ is 1, OCSMACS-GCA can suppress $1 \%$ and $12 \%$ more redundant data than OCSMACS-BU+ and OCSMACSTD+, respectively. It is also worth mentioning that OCSMACS-BU+'s ability to remove redundant data is much better than that of OCSMACS-TD+. The reason is that Bottom-Up scheduling increases the chances of receiving more data hence before performing aggregation. 


\begin{tabular}{|c|c|c|c|c|c|c|}
\hline & \multicolumn{2}{|c|}{ OCSMACS-GCA } & \multicolumn{2}{c|}{ OCSMACS-BU+ } & \multicolumn{2}{c|}{ OCSMACS-TD+ } \\
\hline \hline$\rho$ & Total & SEND $_{h(1)}$ & Total & SEND $_{h(1)}$ & Total & SEND $_{h(1)}$ \\
\hline 0.1 & 193.50 & 100 & 168.75 & 100 & 128.90 & 100 \\
\hline 0.2 & 193.20 & 100 & 167.45 & 100 & 128.55 & 100 \\
\hline 0.3 & 193.30 & 99.95 & 167.90 & 100 & 128.60 & 100 \\
\hline 0.4 & 152.90 & 73.25 & 168.35 & 100 & 128.40 & 100 \\
\hline 0.5 & 134.05 & 62.90 & 167.90 & 100 & 128.50 & 100 \\
\hline 0.6 & 114.75 & 54.05 & 167.40 & 100 & 129.15 & 100 \\
\hline 0.7 & 100.55 & 44.70 & 168.90 & 100 & 128.90 & 100 \\
\hline 0.8 & 84.25 & 35.65 & 168.45 & 100 & 128.45 & 100 \\
\hline 0.9 & 68.80 & 26.25 & 169.50 & 100 & 129 & 100 \\
\hline 1 & 53.80 & 16.85 & 167.80 & 100 & 128 & 100 \\
\hline
\end{tabular}

Table 6.1: Average number of slots $\tau$ required to schedule 100 nodes for different correlation parameter values. $\mathrm{SEND}_{h(1)}$ is the number of SEND slots assigned to nodes in the first hop.

OCSMACS-GCA's efficient redundant data suppression leads to improvements in energy consumption and energy efficiency. Figures 6.2(c) and 6.2(d) show that when $\rho$ is 0.1 , OCSMACS-GCA reduces energy by $5 m J$ and $93 m J$, and increases energy efficiency by $5 \%$ and $18 \%$ compared to OCSMACS-BU+ and OCSMACSTD + , respectively. When $\rho$ is 1 , OCSMACS-GCA is by $15 \%$ and $58 \%$ more energy efficient than OCSMACS-BU+ and OCSMACS-TD+, respectively.

Figure 6.3 shows the energy gain of the $1^{\text {st }}$ hop nodes when OCSMACS-GCA is used compared to OCSMACS-BU+ and OCSMACS-TD+. When $\rho$ is 1, OCSMACSGCA reduces energy consumption by $17 \%$ and $43 \%$ compared to OCSMACS-BU+ and OCSMACS-TD + , respectively. A low to moderate data correlation, e.g. $\rho$ is less than 4 , results in less than $15 \%$ energy gain.

Figure 6.4 shows how each protocol affects energy consumption of the radio and MCU under high and low data correlation levels. With $\rho$ increasing from $0.1 \%$ to $1 \%$, OCSMACS-GCA saves about $26 \mathrm{~mJ}$ of radio energy consumption and $16 \mathrm{~mJ}$ of the 


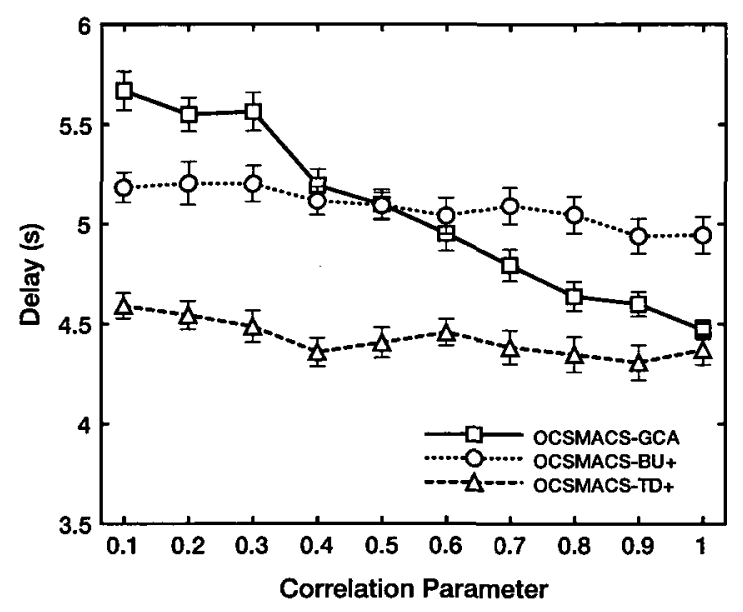

(a) Delay

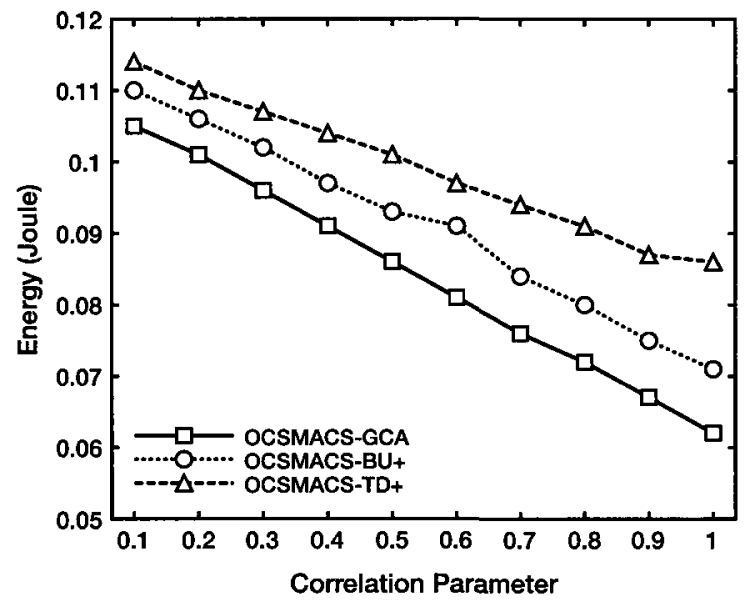

(c) Energy

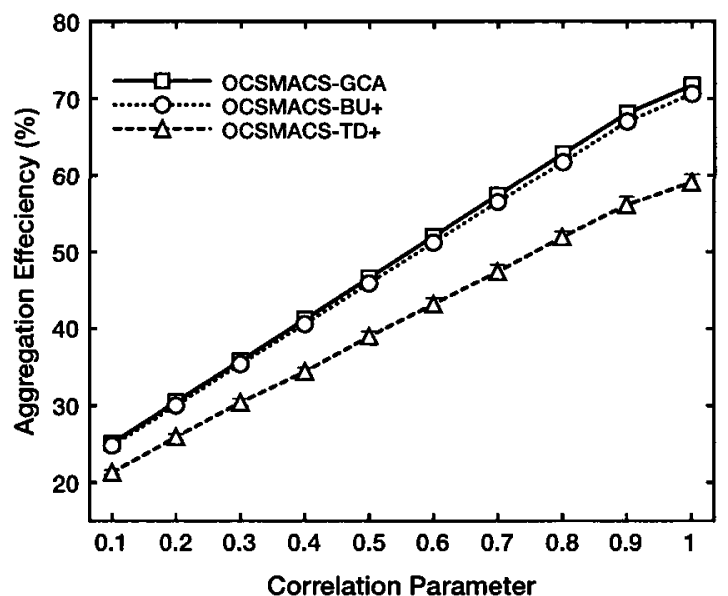

(b) Aggregation efficiency

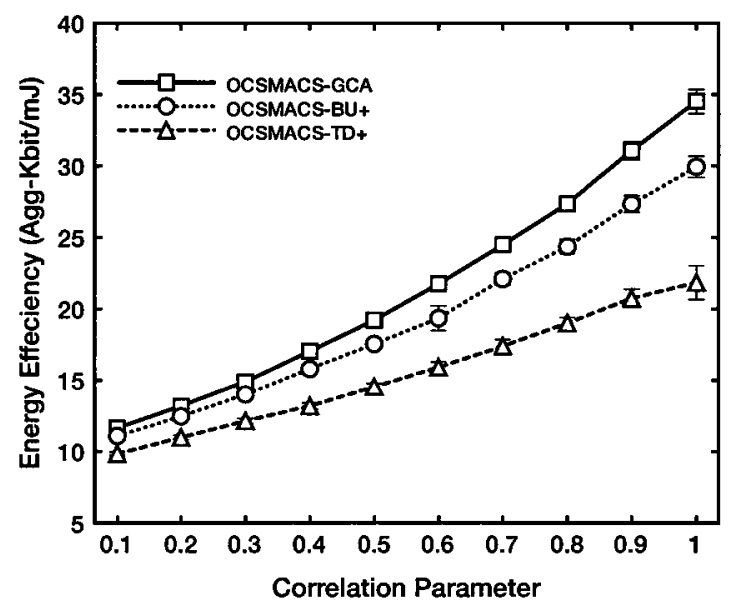

(d) Energy efficiency

Figure 6.2: Simulation results of OCSMACS-GCA, OCSMACS-BU+ and OCSMACS$\mathrm{TD}+$ for variable correlation parameter $(\rho)$. 


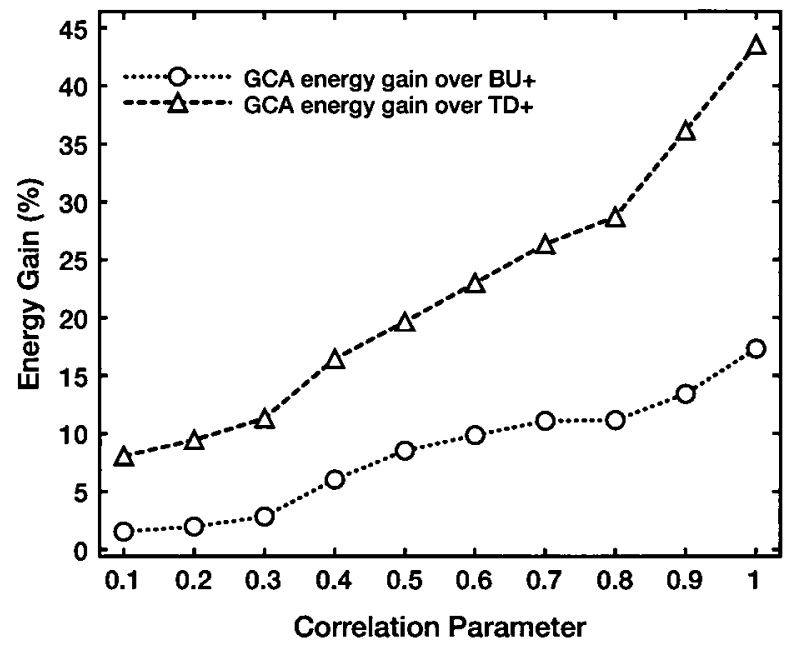

Figure 6.3: Energy gain using the GCA Scheduling instead of TD and BU scheduling.

MCU. OCSMACS-BU+ saves $24 m J$ of the radio energy and $14 m J$ of the MCU. On the other hand, OCSMACS-TD+ saves $18 \mathrm{~mJ}$ of the radio energy consumption and $10 \mathrm{~mJ}$ of the MCU. Considering the idle listening energy only, OCSMACS-GCA and OCSMACS-BU+ reduce it by $7 \%$ and $4 \%$, respectively. OCSMACS-TD+, however, increases it by $1.8 \%$. This is a result of OCSMACS-TD+ assigning more RECEIVE slots than actually used, compared to other protocols.

Correlation-aware OCSMACS's network lifetime is determined using a scenario similar to that in Section 5.4.1 where 100 nodes are simulated and $r_{\text {sampling }}$ is 0.003 pkt/sec. Figure 6.5 shows lifetimes of OCSMACS-GCA, OCSMACS-BU+ and OCSMACSTD+. It can be observed that indeed, designing the scheduling scheme based on knowledge of data correlation results in extending network lifetime. For example when $\rho$ is 1 , OCSMACS-GCA extends network lifetime by 0.26 and 0.51 years compared to OCSMACS-TD+ and OCSMACS-BU+, respectively. In addition, this is about one year longer than noncorrelation-aware OCSMACS. 


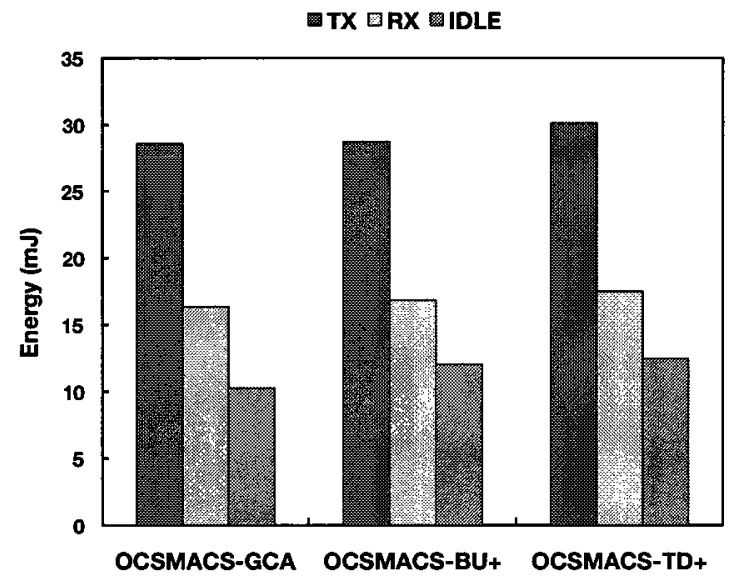

(a) Radio energy $(\rho=0.1)$

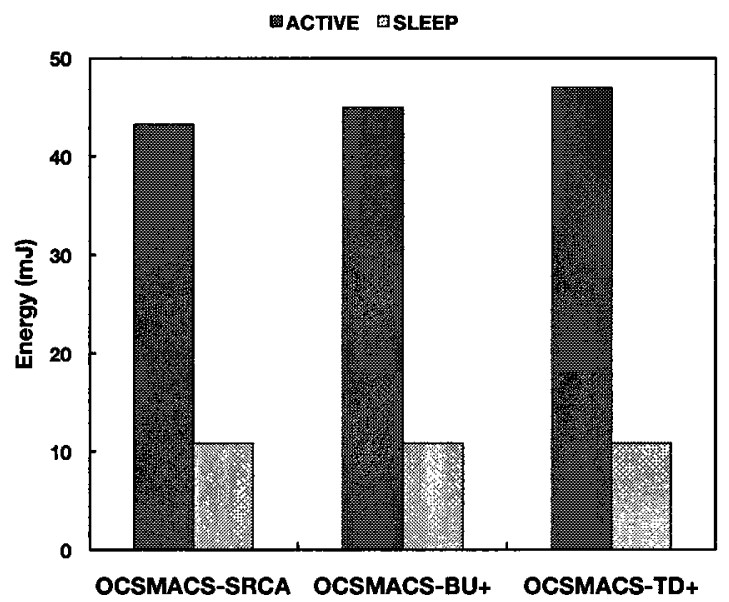

(c) MCU energy $(\rho=0.1)$

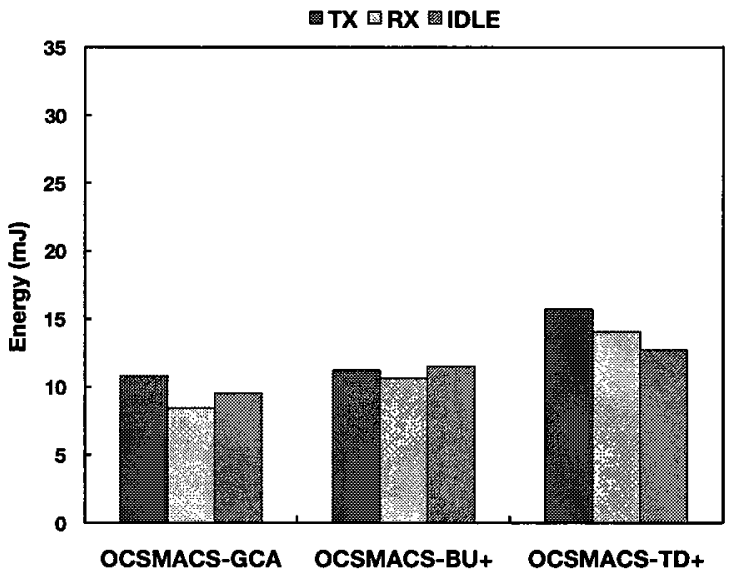

(b) Radio energy $(\rho=1)$

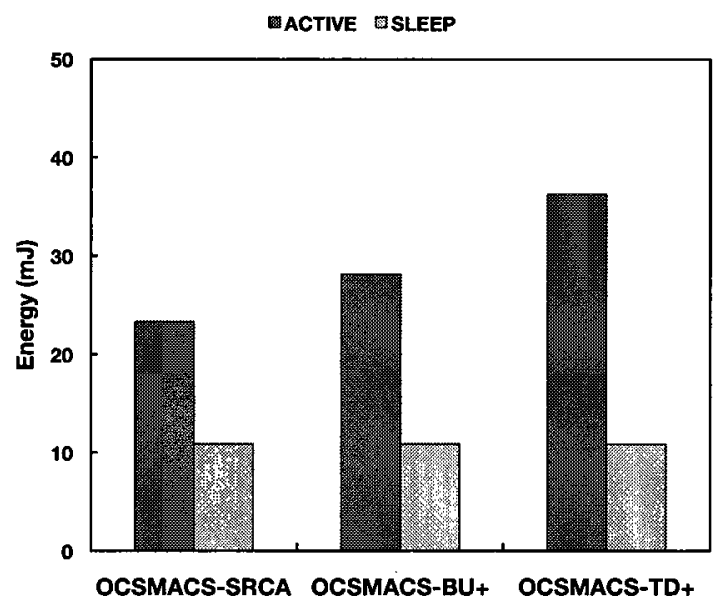

(d) MCU energy $(\rho=1)$

Figure 6.4: Radio and MCU energy consumption. 


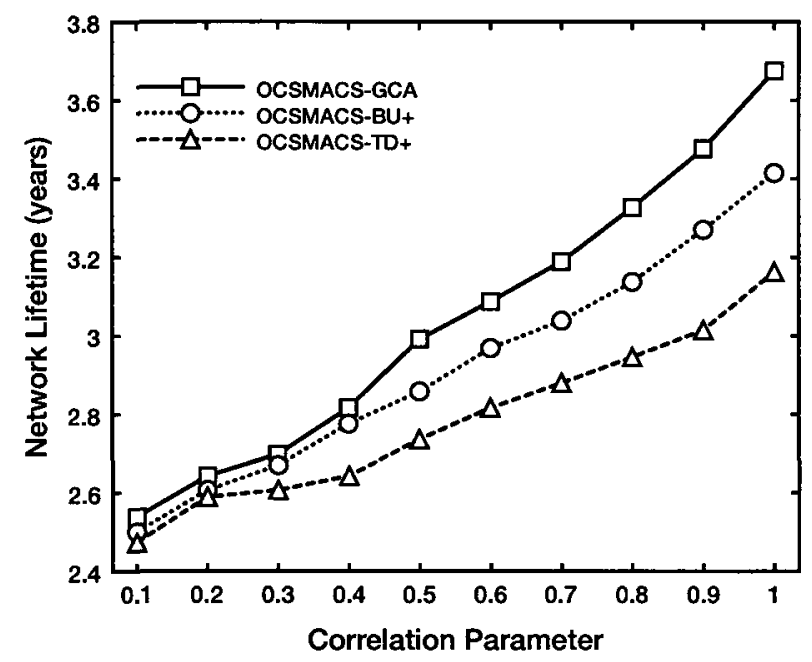

Figure 6.5: Network lifetime, $r_{\text {sampling }}=0.003 \mathrm{pkt} / \mathrm{sec}$.

\subsubsection{OCSMACS-SRCA}

This section evaluates the performance of OCSMAC with the Sensing-range Correlationaware scheduling under a variable sensing range and compares it with that of OCSMACSBU+ and OCSMACS-TD+. A network of 100 nodes with neighboring nodes separated uniformly in [10-60] $m$ is simulated. The maximum transmission range is $60 m$ while the sensing range is varying from 5 to $65 \mathrm{~m}$. Increasing the maximum transmission range requires an increaser in the transmit power, therefore, the radio transmit current is increased from $16.5 \mathrm{~mA}$ to $26.7 \mathrm{~mA}$. In addition, the aggregation processing time is assumed to be $5 \mathrm{~ms}$. Table 6.2 shows the suggested values of $\rho$ for different ranges of $d\left(s_{0}, s_{1}\right)$.

The simulation results shown in Figure 6.6 indicates an increasing trend in aggregation efficiency, energy and energy efficiency as $\mathcal{R}_{\text {sense }}$ approaches $\mathcal{R}_{s n}$. In Figure 6.6(b), a sensing range below the minimum node separation of $10 \mathrm{~m}$ results in all data being uncorrelated, hence, aggregation does not takes place. On the other hand, when $\mathcal{R}_{\text {sense }}$ is equivalent to the $\mathcal{R}_{s n}$, OCSMACS-SRCA achieves up to $70 \%$ aggregation 
efficiency, compared to the $67 \%$ and $49 \%$ efficiency archived by OCSMACS-BU+ and OCSMACS-TD+, respectively.

\begin{tabular}{|c|c|}
\hline Nodes separation $(\mathrm{m})$ & $\rho_{s_{0}, s_{1}}$ \\
\hline$d\left(s_{0}, s_{1}\right) \geq 2 \mathcal{R}_{\text {sense }}$ & 0 \\
\hline $2 \mathcal{R}_{\text {sense }}>d\left(s_{0}, s_{1}\right) \geq 1.8 \mathcal{R}_{\text {sense }}$ & 0.2 \\
\hline $1.8 \mathcal{R}_{\text {sense }}>d\left(s_{0}, s_{1}\right) \geq 1.6 \mathcal{R}_{\text {sense }}$ & 0.4 \\
\hline $1.6 \mathcal{R}_{\text {sense }}>d\left(s_{0}, s_{1}\right) \geq 1.4 \mathcal{R}_{\text {sense }}$ & 0.6 \\
\hline $1.4 \mathcal{R}_{\text {sense }}>d\left(s_{0}, s_{1}\right) \geq 1.2 \mathcal{R}_{\text {sense }}$ & 0.8 \\
\hline$d\left(s_{0}, s_{1}\right)<1.2 \mathcal{R}_{\text {sense }}$ & 1 \\
\hline
\end{tabular}

Table 6.2: Suggested values of $\rho$ as the distance between nodes changes

The improvement in aggregation efficiency results in energy savings and better energy efficiency. OCSMACS-SRCA transports about 31.66 Kbit of aggregated data using $1 m J$ when $\mathcal{R}_{\text {sense }}$ is equivalent to $\mathcal{R}_{s n}$. This is by $5.4 \mathrm{Kbit}$ and $15.3 \mathrm{Kbit}$ more compared to OCSMACS-BU+ and OCSMACS-TD+, respectively.

When $\mathcal{R}_{\text {sense }}$ is $5 m$, OCSMACS-SRCA increases the end-to-end delay by $0.3 \mathrm{~s}$ and 1s compared to OCSMACS-BU+ and OCSMACS-TD+, respectively. Despite that, once $\mathcal{R}_{\text {sense }}$ exceeds $45 \mathrm{~m}$ OCSMACS-SRCA reduces delay rapidly until it becomes within the margin of error with OCSMACS-TD+'s delay. Similar to OCSMACSGCA, this reduction in delay is because OCSMACS-SRCA reduces the number of slots as the difference between $\mathcal{R}_{\text {sense }}$ and $\mathcal{R}_{s n}$ decreases (Table 6.3).

First hop nodes' achievable energy gain, if OCSMACS-SRCA is used instead of OCSMACS-BU+ or OCSMACS-TD+, is shown in Figure 6.7. An $\mathcal{R}_{\text {sense }}$ of $5 \mathrm{~m}$ does not result in any gain. However, energy gain continues to increase as $R_{\text {sense }}$ increases. Compared to OCSMACS-BU+ and OCSMACS-TD+, OCSMACS-SRCA reduces energy by $24 \%$ and $57 \%$, respectively, when $\mathcal{R}_{\text {sense }}$ is $55 \mathrm{~m}$. 


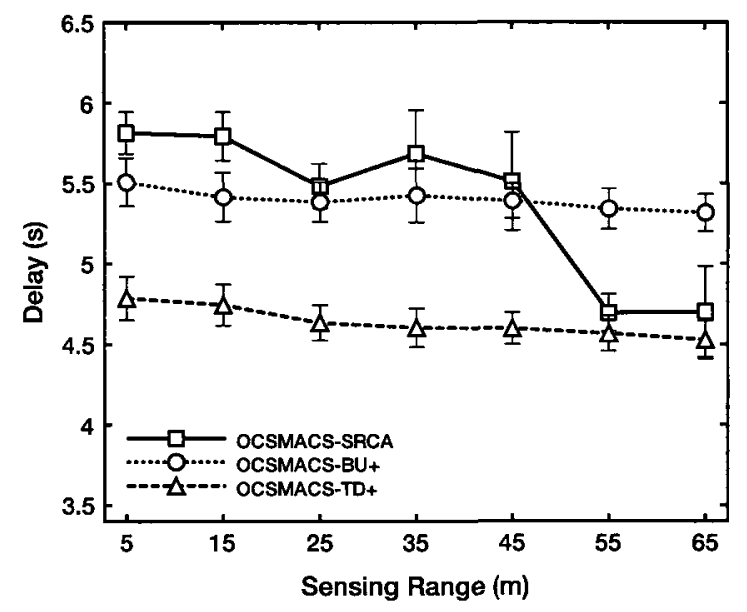

(a) Delay

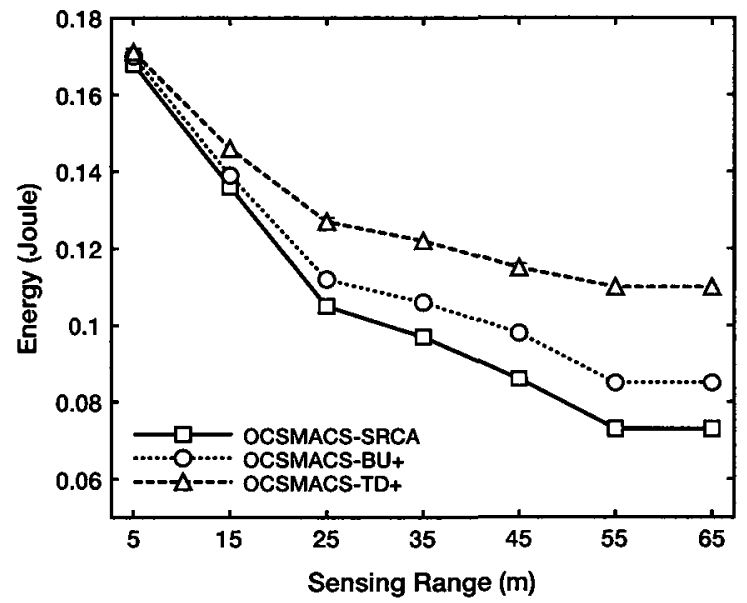

(c) Energy

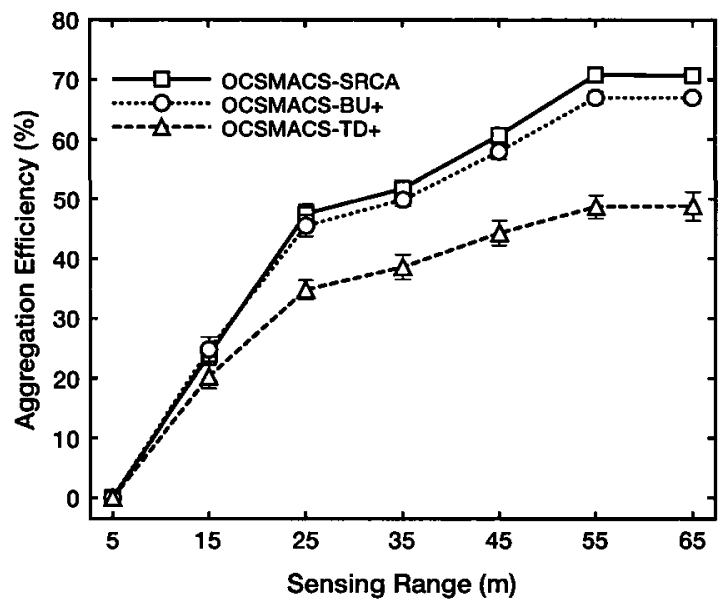

(b) Aggregation efficiency

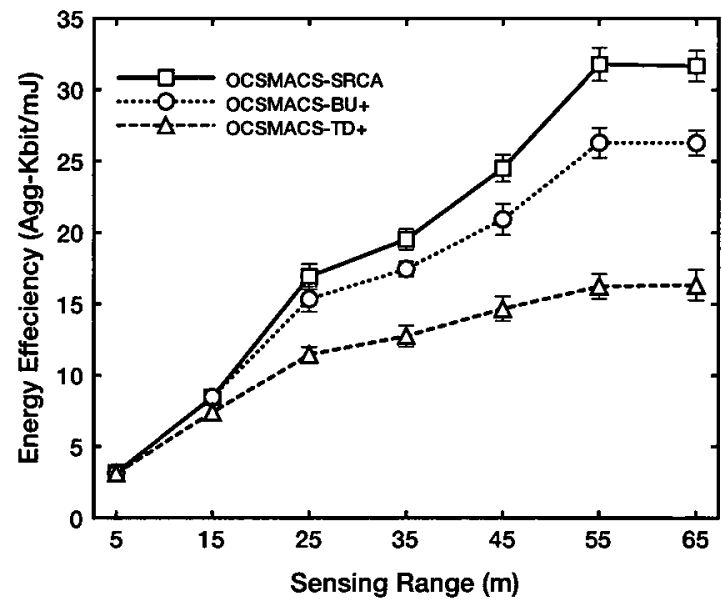

(d) Energy efficiency

Figure 6.6: Simulation results of OCSMACS-SRCA, OCSMACS-BU+, and OCSMACS$\mathrm{TD}+$ for a variable sensing range. 


\begin{tabular}{|c|c|c|c|c|c|c|}
\hline & \multicolumn{2}{|c|}{ OCSMACS-SRCA } & \multicolumn{2}{c|}{ OCSMACS-BU+ } & \multicolumn{2}{c|}{ OCSMACS-TD+ } \\
\hline \hline $\mathcal{R}_{\text {sense }}$ & Total & SEND $_{h(\mathbf{1})}$ & Total & SEND $_{h(1)}$ & Total & SEND $_{h(1)}$ \\
\hline 5 & 211.55 & 100 & 180.80 & 100 & 140.90 & 100 \\
\hline 15 & 204.10 & 96.40 & 181.35 & 100 & 141 & 100 \\
\hline 25 & 159.60 & 72.25 & 181.40 & 100 & 140.90 & 100 \\
\hline 35 & 132.70 & 58.85 & 181.75 & 100 & 140.85 & 100 \\
\hline 45 & 101.25 & 41.05 & 180.70 & 100 & 140.85 & 100 \\
\hline 55 & 61.45 & 20.55 & 180 & 100 & 140.90 & 100 \\
\hline 65 & 61.25 & 20.65 & 181.95 & 100 & 140.90 & 100 \\
\hline
\end{tabular}

Table 6.3: Number of slots $\tau$ required to schedule 100 nodes for variable sensing ranges $\mathcal{R}_{\text {sense }}$ SEND $_{h(1)}$ is the number of SEND slots assigned to nodes in the first hop.

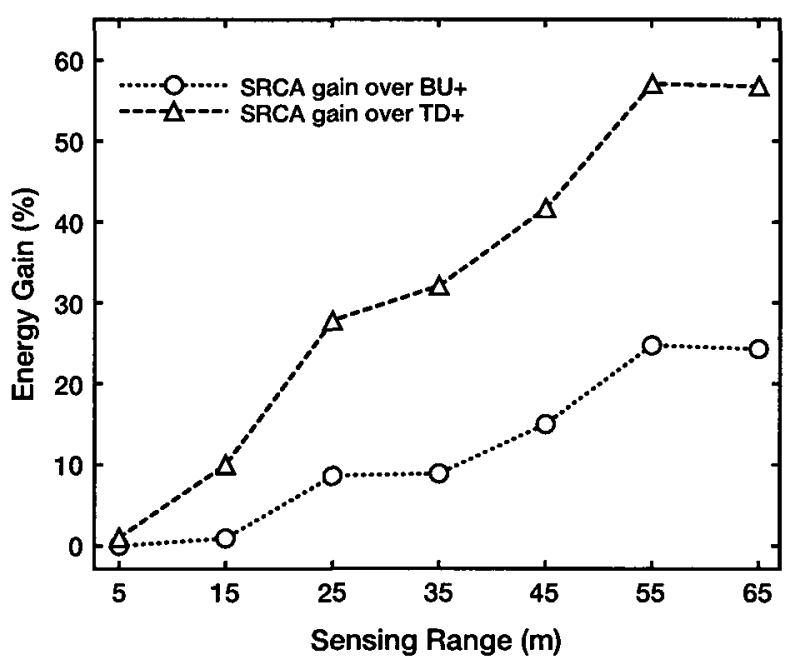

Figure 6.7: Energy gain using the Correlation-aware scheduling instead of Top-Down or Bottom-Up scheduling.

Figure 6.8 shows the energy consumed by the radio and the MCU for $\mathcal{R}_{\text {sense }}$ of $15 \mathrm{~m}$ and $65 \mathrm{~m}$. OCSMACS-SRCA's combined radio transmit/receive energy is about $160 \mathrm{~mJ}$ at a $15 \mathrm{~m}$ sensing range and about $113 \mathrm{~mJ}$ at a $65 \mathrm{~m}$ sensing range. Compared to OCSMACS-BU+, this is equivalent to $1.6 \%$ and $18 \%$ reduction at $15 \mathrm{~m}$ and $65 \mathrm{~m}$ $\mathcal{R}_{\text {sense }}$, respectively. While compared to OCSMACS-TD+, it is equivalent to $7 \%$ and $63 \%$ reduction at $15 m$ and $65 m \mathcal{R}_{\text {sense }}$, respectively. With respect to idle listening 
energy, OCSMACS-SRCA, OCSMACS-BU+ and OCSMACS-TD+ reduce it by $25 \%$, $18 \%$ and $9 \%$, respectively.

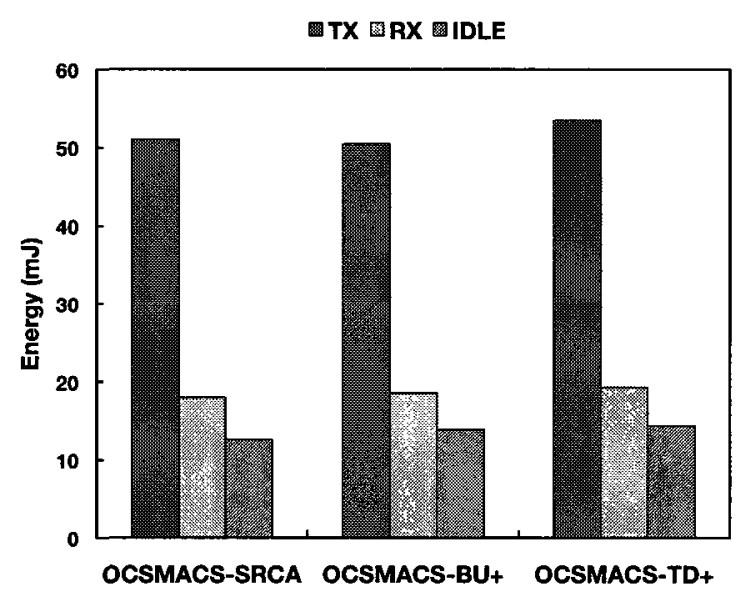

(a) Radio energy $\left(\mathcal{R}_{\text {sense }}=15 \mathrm{~m}\right)$

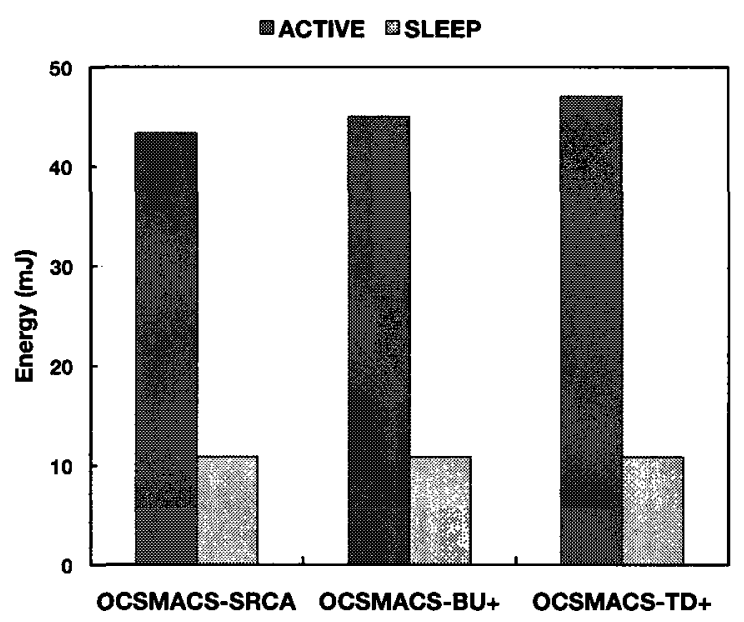

(c) MCU energy $\left(\mathcal{R}_{\text {sense }}=15 \mathrm{~m}\right)$

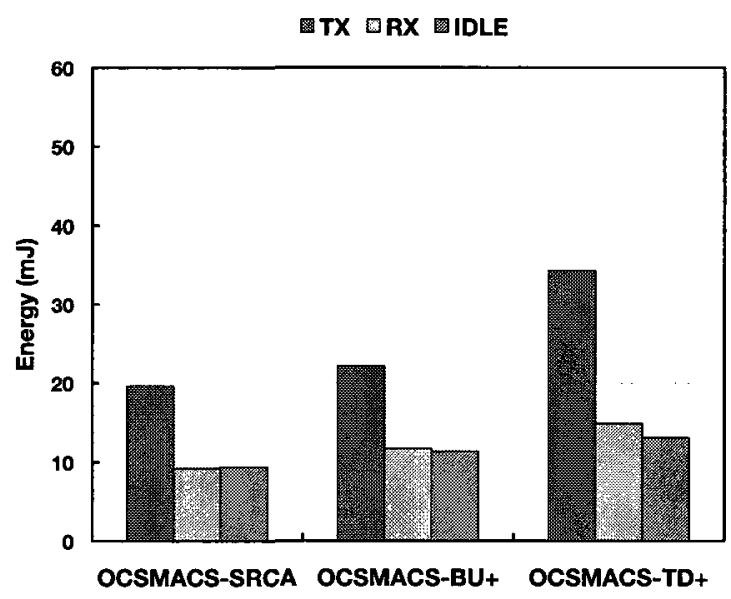

(b) Radio energy $\left(\mathcal{R}_{\text {sense }}=65 \mathrm{~m}\right)$

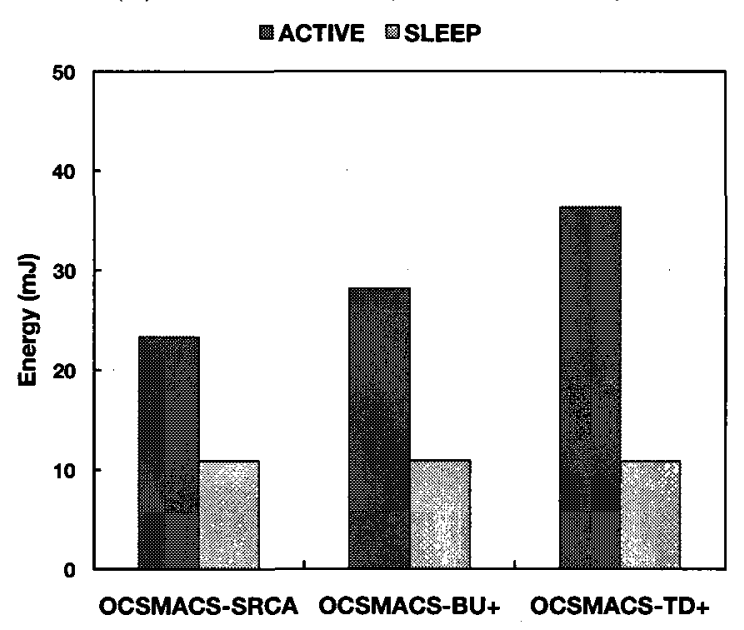

(d) $\mathrm{MCU}$ energy $\left(\mathcal{R}_{\text {sense }}=65 \mathrm{~m}\right)$

Figure 6.8: Radio and MCU energy consumption.

Network lifetime of OCSMACS-SRCA is shown in Figure 6.9. Similar to previous OCSMACS-SRCA results, as $\mathcal{R}_{\text {sense }}$ gets closer to the transmission range more data becomes correlated, better aggregation and, therefore, longer network lifetime. The figure shows that, when $\mathcal{R}_{\text {sense }}$ and $\mathcal{R}_{\text {sn }}$ are the same, OCSMACS-SRCA extends 
network lifetime by 0.4 and 1 years compared to OCSMACS-BU+ and OCSMACS$\mathrm{TD}+$, respectively.

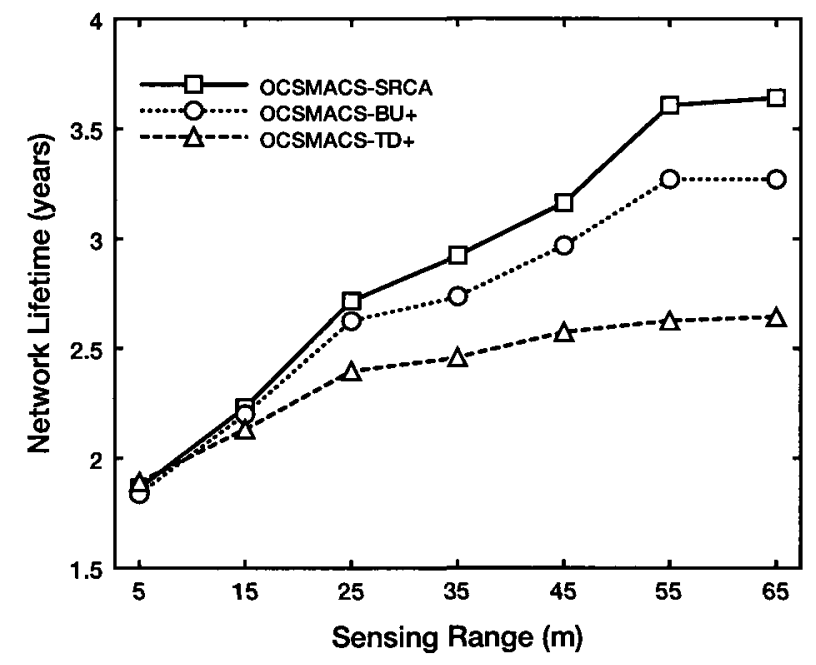

Figure 6.9: Network lifetime, $r_{\text {sampling }}=0.003 \mathrm{pkt} / \mathrm{sec}$.

\subsubsection{Aggregation Feasibility}

The time it takes to perform the aggregation process depends on the sensing task, data, and complexity of the running algorithm. If the computational energy exceeds the gain achieved by aggregation then, it is better to relay data as is and leave the aggregation process to the sink or a remote processing entity which utilizes the data for decision making. In addition, because of the processing time requirement, correlation-aware scheduling increases the delay compared to the other proposed scheduling mechanisms. For applications with very short delay requirements, in-network data aggregation may not be desirable.

In this section, simulations are used to find the conditions under which in-network data aggregation is a feasible solution for improving energy efficiency. In other words, the aim is to determine the maximum achievable energy gain before the aggregation 
process becomes another source of undesirable energy waste. For that reason, 100 nodes and varying aggregation processing time $T_{\text {aggregation }}$ in $[20-50] m s$ are simulated. Both OCSMACS-GCA and OCSMACS-SRCA are compared against OCSMAC-TD and OCSMACS-BU.

Figure 6.10 shows that increasing $T_{\text {aggregation }}$ increases energy consumption of OCSMACS-GCA. At low correlation levels, e.g. when $\rho$ is less than 0.5 , a $40 \mathrm{~ms}$ processing time increases OCSMACS-GCA's energy consumption beyond that of OCSMACS-BU. Even when $\rho$ is 1 , the achievable gain is less than $0.3 J$ of energy. Using $40 \mathrm{~ms}$ processing time, OCSMACS-GCA performs better than OCSMACS-TD only when $\rho$ is greater than 0.5 . Therefore, if $T_{\text {aggregation }}$ is more than $40 \mathrm{~ms}$ then OCSMACS-GCA is less likely to achieve energy gain compared to OCSMACS-BU. Instead it may consume more energy and cause longer delays. Similarly, if compared to OCSMACS-TD, it could consume more energy except when data is highly correlated.

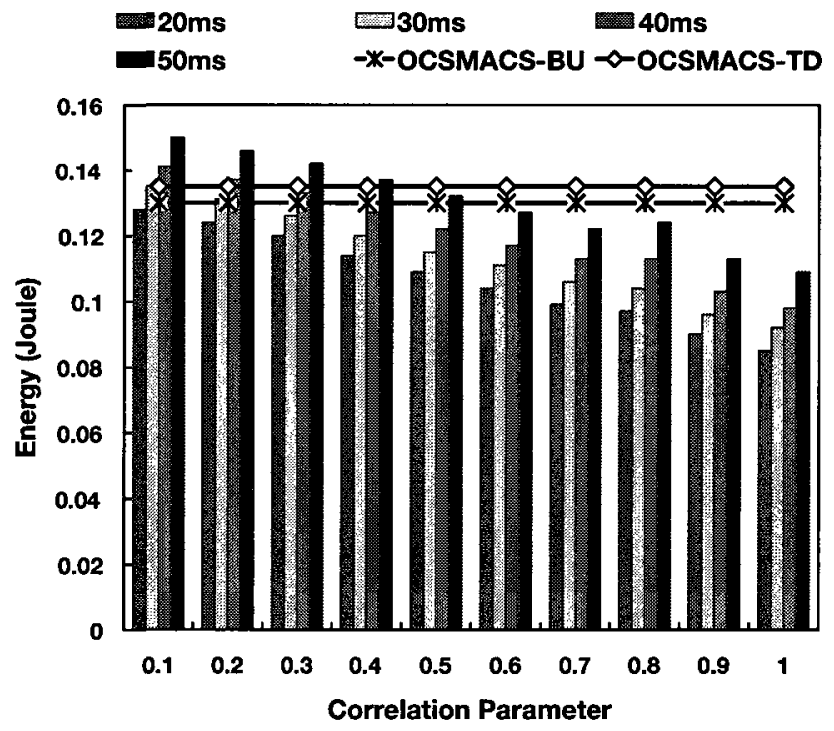

Figure 6.10: Energy consumption of OCSMACS-GCA vs. correlation parameter $(\rho)$ for different aggregation times $\left(T_{\text {aggregation }}\right)$. 
Unlike OCSMACS-GCA, OCSMACS-SRCA is severely affected by increasing the processing time $T_{\text {aggregation }}$ (Figure 6.11). It can be observed that if $\mathcal{R}_{\text {sens }}$ is less than $45 m$ OCSMACS-SRCA consumes the same or more energy than OCSMACS-TD and OCSMACS-BU. Only when $\mathcal{R}_{\text {sense }}$ is beyond $45 \mathrm{~m}$ OCSMACS-SRCA achieves some energy gain.

Aggregation feasibility can also be used to study the energy cost when the energy consumption increases with an increase in the required level of data redundancy reduction or with the increase in the required level of data compression. For this purpose, simulations of 100 nodes are carried out. $T_{\text {aggregation }}$ (translates to aggregation energy) increases linearly in $[0-80] \mathrm{ms}$. The increase is triggered when the desired level of aggregation/compression increases. Figure 6.12 shows the communication $(\mathrm{TX}+\mathrm{RX}), \mathrm{MCU}$ and overall energy consumption versus the data aggregation energy. The figure shows that the maximum energy saving of almost $50 \%$ is achievable when $2.8 \mathrm{~mJ}$ of processing energy is consumed to compress/suppress $60 \%$ of the transmitted data. Removing all data redundancy or compressing data packets from multiple sources in a single packet, however, reduces overall energy consumption by only $39 \%$. The reason is that while the TX+RX energy is reduced by $71 \%$, the $\mathrm{MCU}$ and the radio idle energy is reduced by only $36 \%$ and $3 \%$, respectively. Unlike the $\mathrm{TX}+\mathrm{RX}$ energy, the idle energy increases as the processing time increases because the number of of ScheduleReq slots is increased to satisfy the frame length condition that is previously discussed in Section 3.4. 


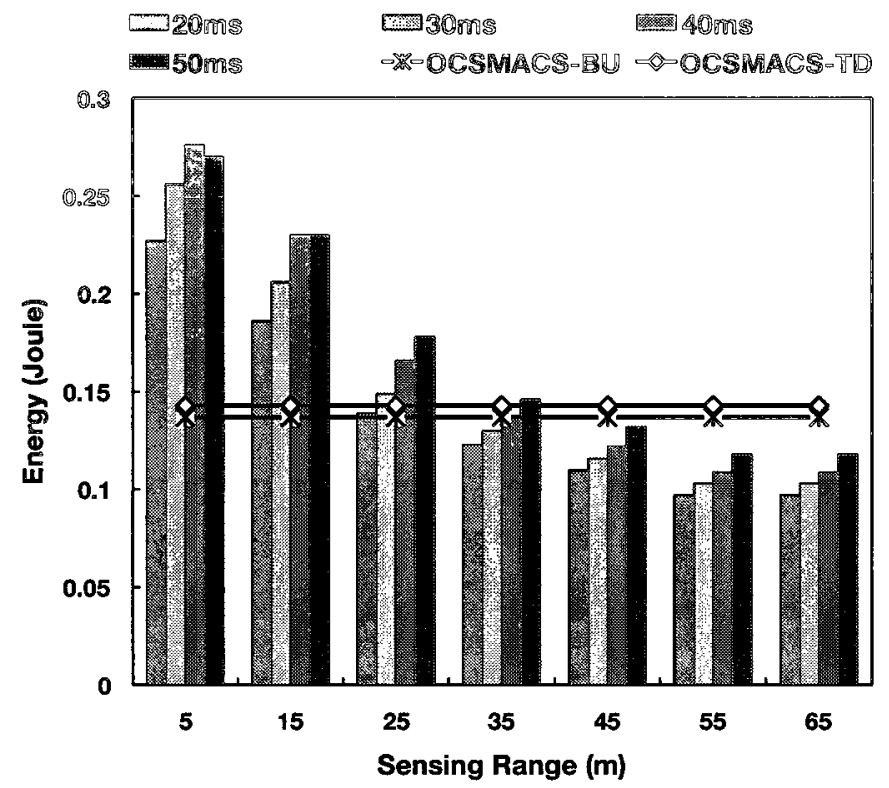

Figure 6.11: Energy consumption of OCSMACS-SRCA vs. sensing range for different aggregation times ( $\left.T_{\text {aggregation }}\right)$.

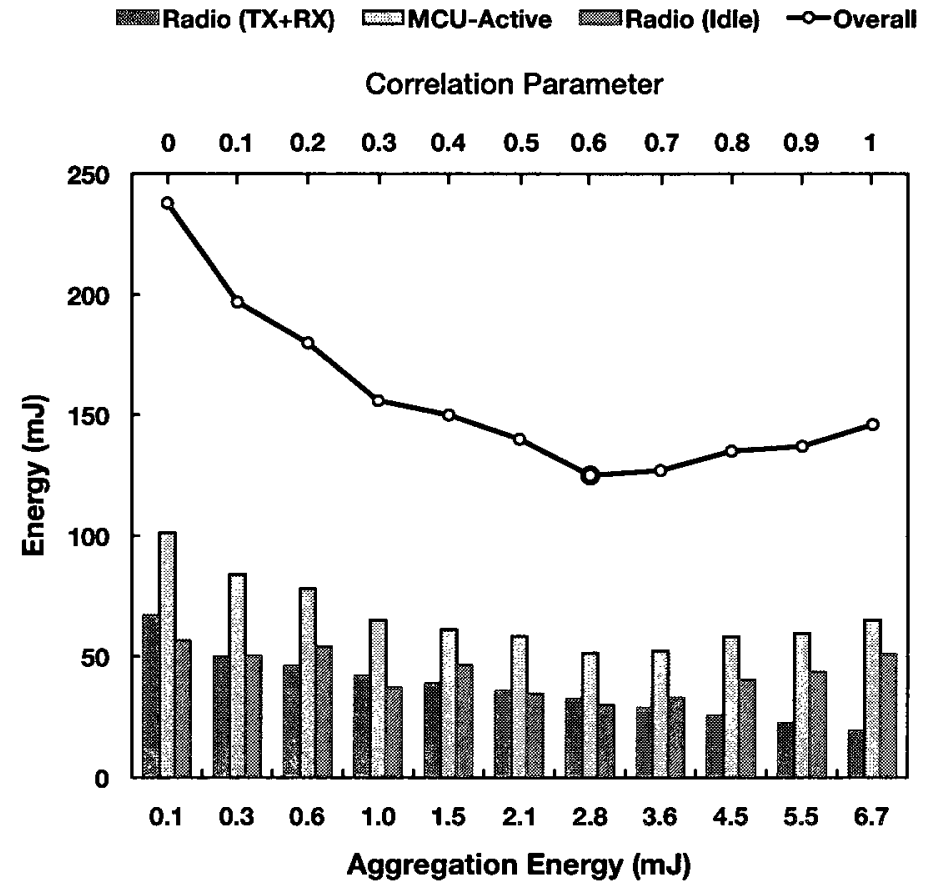

Figure 6.12: Communication, processing, and overall energy consumption of OCSMACSGCA for different aggregation processing/compression energy. 


\subsection{Summary}

Previous work on data aggregation and fusion in WSNs focuses on higher level design aspects, e.g. routing and clustering. Nevertheless, the underlying MAC protocol has a major impact on energy efficiency and network lifetime. In this chapter, the potential energy gain achieved by utilizing a knowledge of data correlation to design multihop scheduling schemes is studied. Two scheduling schemes: the first one is based on a global data correlation model and the second one is based on a more realistic model where data correlation exists if sources are within sensing range of each other are proposed.

Simulation results show that OCSMACS together with the proposed correlationaware scheduling improves energy efficiency and extends network lifetime. In the case of GCA scheduling when $\rho$ is 1 , OCSMACS-GCA extends network lifetime by 0.26 and 0.51 years compared to OCSMACS-TD+ and OCSMACS-BU+, respectively. OCSMACS-SRCA performs better by extending network lifetime by 0.4 and 1 years compared to OCSMACS-BU+ and OCSMACS-TD+, respectively, when the sensing and transmission ranges are the same. 


\section{Chapter 7}

\section{Conclusions and Future Work}

Energy efficient MAC design presents many challenges in wireless sensor networks. We consider sensor networks with convergecast (many-to-one) type of applications. In addition, data aggregation and compression are used to reduce redundancy and to provide a solution for adaptive slot assignment. This dissertation focused on multihop scheduling-based (TDMA) MAC protocols which facilitate the follow of data from sources to sink.

It is well-known that convergecast traffic might cause congestion at nodes close to the sink. In addition, a conventional TDMA protocol has a number of drawbacks which make it undesirable for certain sensor applications and/or network configurations. Examples of such drawbacks are complex slot allocation and schedule maintenance, effective avoidance of idle listening, tight global clock synchronization and low channel utilization in low traffic conditions. In this dissertation, we concentrated on adaptive and correlation-aware scheduling schemes which minimize or eliminate the effect of these drawbacks to achieve specific application requirements (energy efficiency and delay). 
The main conclusions and suggested future work for each part are summarized below.

1. PROGRESSIVE - a protocol which schedules nodes in a progressive fashion so that nodes are gradually assigned transmission slots, has been proposed.

- The optimal NET-LisT backoff window size is determined through simulation. It has been observed that the best size that maximizes the number of nodes which are scheduled at the end of the topology collection phase depends on the network size, density, and number of hops.

- The effects of various protocol and network specific parameters; discovery and topology collection phases duration, network size and network density on the total number of scheduled nodes (scheduling efficiency), scheduling time and energy have been studied. Results have shown that the novel design of PROGRESSIVE makes it very time and energy efficient topology discovery and scheduling setup protocol compared to DRAND. It reduces energy consumption by a considerable factor compared to DRAND for variable network sizes. With respect to relative network density, PROGRESSIVE maintains very low energy consumption compared to DRAND in which energy consumption increases sharply by increasing the relative density.

- Possible future work for this topic my target the design of a hybrid PROGRESSIVE which utilizes progressive scheduling during the initial network setup then uses a distributed protocol, similar to DRAND, during normal network operation. A PROGRESSIVE-then-distributed protocol would 
be an excellent solution that combine the advantages of both fast energy efficient network setup and scalability.

2. An On-demand Convergecast Scheduling-based MAC (OCSMACS) has been proposed. OCSMACS addressed the identified TDMA MAC issues by using the following mechanism:

- A very adaptive on-demand slot assignment has been proposed. While usually used for data redundancy reduction, aggregation is the main underlying mechanism that is used to aggregate many slot assignment requests in a single compact one. Requests aggregation is based on the assumption that in a short time interval large number of requests is generated by nodes in close proximity.

- Based on maximum application's end-to-end delay requirement, scheduling, delay, schedule request duration, schedule computing time and resynchronization frequency, the optimal frame size in terms of the number of data exchange slots has been evaluated. Similarly, network lifetime maximization has been modeled. The objective is to maximize the frame size subject to maximizing data delivery and preventing queues overflow.

- Topology information collected during PROGRESSIVE enables integration of the routing and MAC as a single protocol. Two interleaved spatialtemporal scheduling schemes have been proposed: Top-Down scheduling and Bottom-Up scheduling. OCSMACS using these scheduling schemes is called OCSMACS-TD and OCSMACS-BU, respectively. It has been observed that Top-Down scheduling is more likely to have better end-to-end 
delays than Bottom-Up scheduling. While, the opposite is valid in terms of energy efficiency.

- OCSMACS's performances of an application that requires the best energy efficiency and another application requires a one second maximum endto-end delay have been studied using simulations. Optimal frame sizes have been obtained for each application while varying network size and sampling rate.

- Possible future work on this topic includes:

- Different variations of the proposed scheduling schemes can be investigated. For example, in deciding the next node along the route to sink, residual nodes energy, queue size, event type, etc. can be considered. In addition, assigning different transmit powers to nodes to enhance overall energy consumption. Point-to-point (graph coloring) sink-assisted scheduling can be also a topic of future studies.

- Simulation and performance analysis is based on a single cell network configuration. Evaluation of OCSMACS in multicell configuration can be targeted. In such configuration, it is important to coordinate slot assignment among cells and to handle intra and inter cell interference.

3. The potential of data aggregation has been analyzed in the context of multihop scheduling MAC design.

- To be used in correlation-aware scheduling, the Sensing Range Correlation model has been proposed. Based on this model and the Global Correlation model, two correlation-aware scheduling schemes have been proposed, 
SRCA and GCA scheduling. Both schemes are non-interleaved spatial temporal scheduling where transmission slots assignment are delayed until all data received from down stream neighbors.

- It has been observed that integrating the proposed scheduling schemes with OCSMACS, energy efficiency can be significantly improved compared to OCSMACS-TD and OCSMACS-BU even with opportunistic aggregation.

- Given energy consumption of the radio and micro-controller, sampling rate, data packet size, and aggregation parameter or sensing range, the limit on aggregation power while achieving energy gain has been evaluated.

- Future studies on this topic may include enhancing non-interleaved scheduling by considering better correlated routes. 
Appendix A

\section{Scheduling Algorithms}




\section{A.1 Interleaved Spatial-Temporal Scheduling}

Algorithm A.1.1: $\operatorname{ISTS}(G, C$, criteria $)$

$1: \operatorname{nodes}[|V|] \leftarrow \operatorname{sort}(G, \operatorname{criteria})$

2: $\forall A C T I V E(v) \in G$, pktsQueued $(v) \leftarrow 1$

3: $\forall \mathcal{T}$ time-slots and $\forall v \in G, v \leftarrow S L E E P$

for $\mathcal{T}$ time-slots

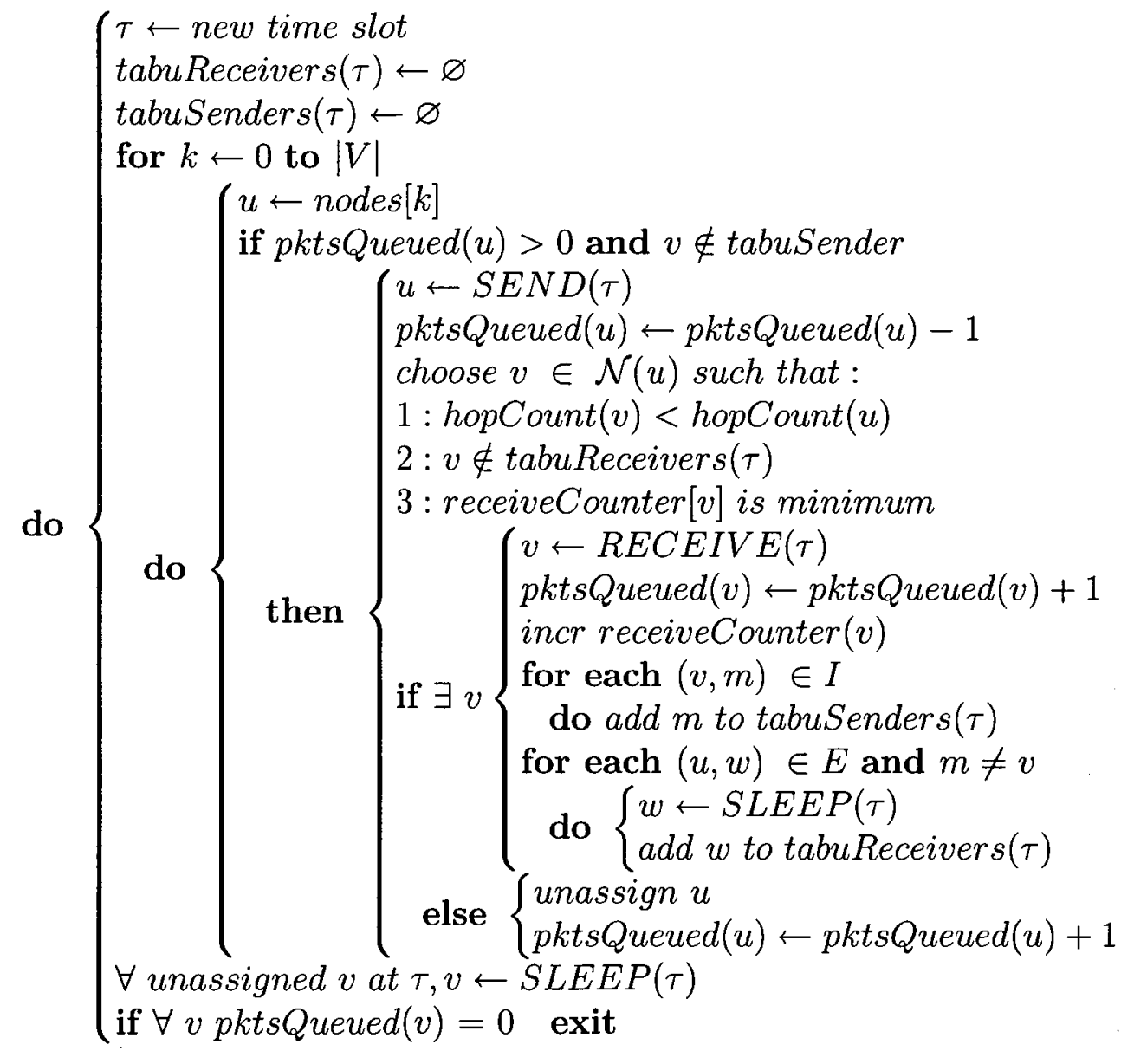




\section{A.2 Leafnode-based Non-interleaved Scheduling}

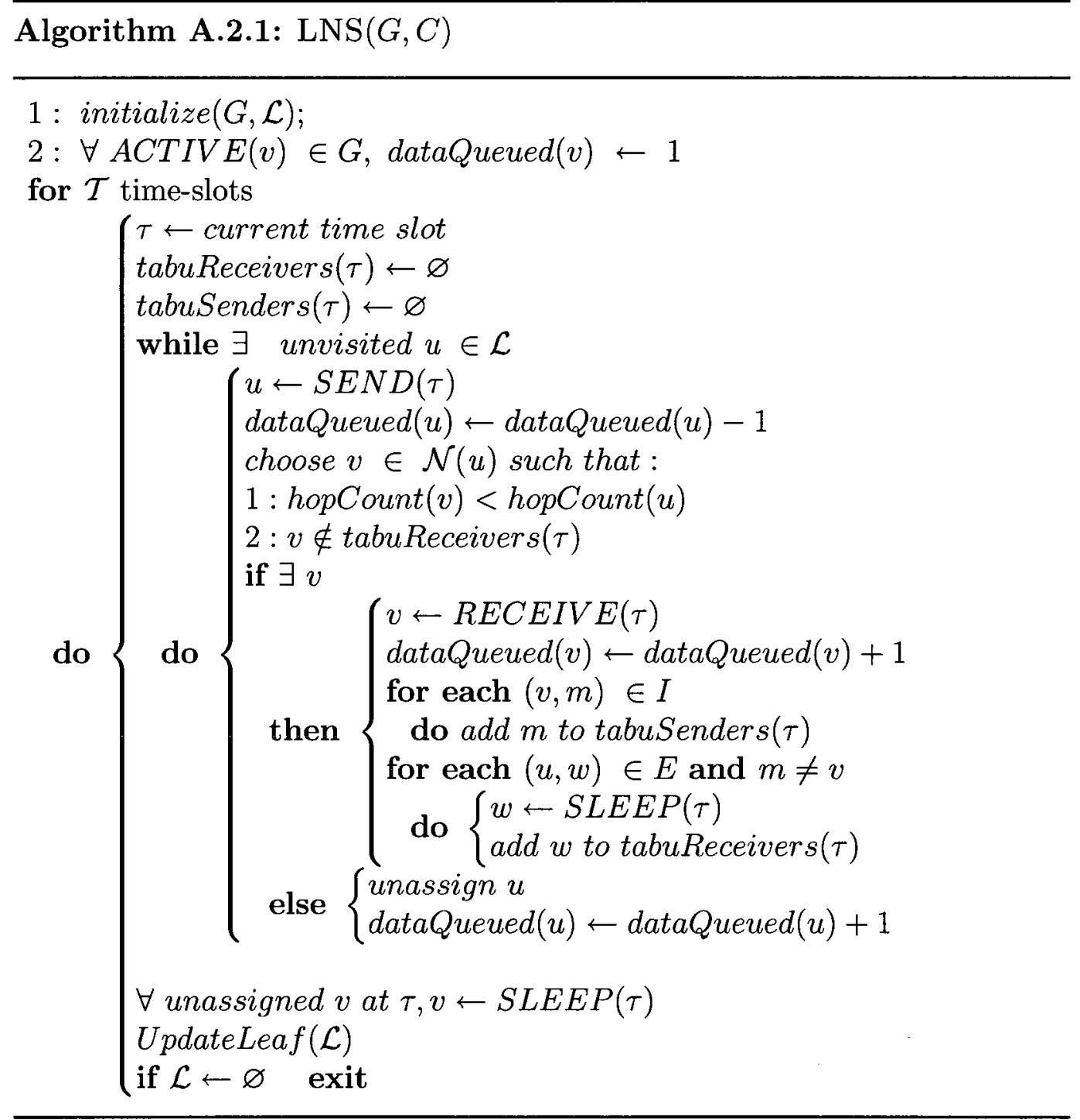




\section{A.3 Global Correlation-aware Scheduling}

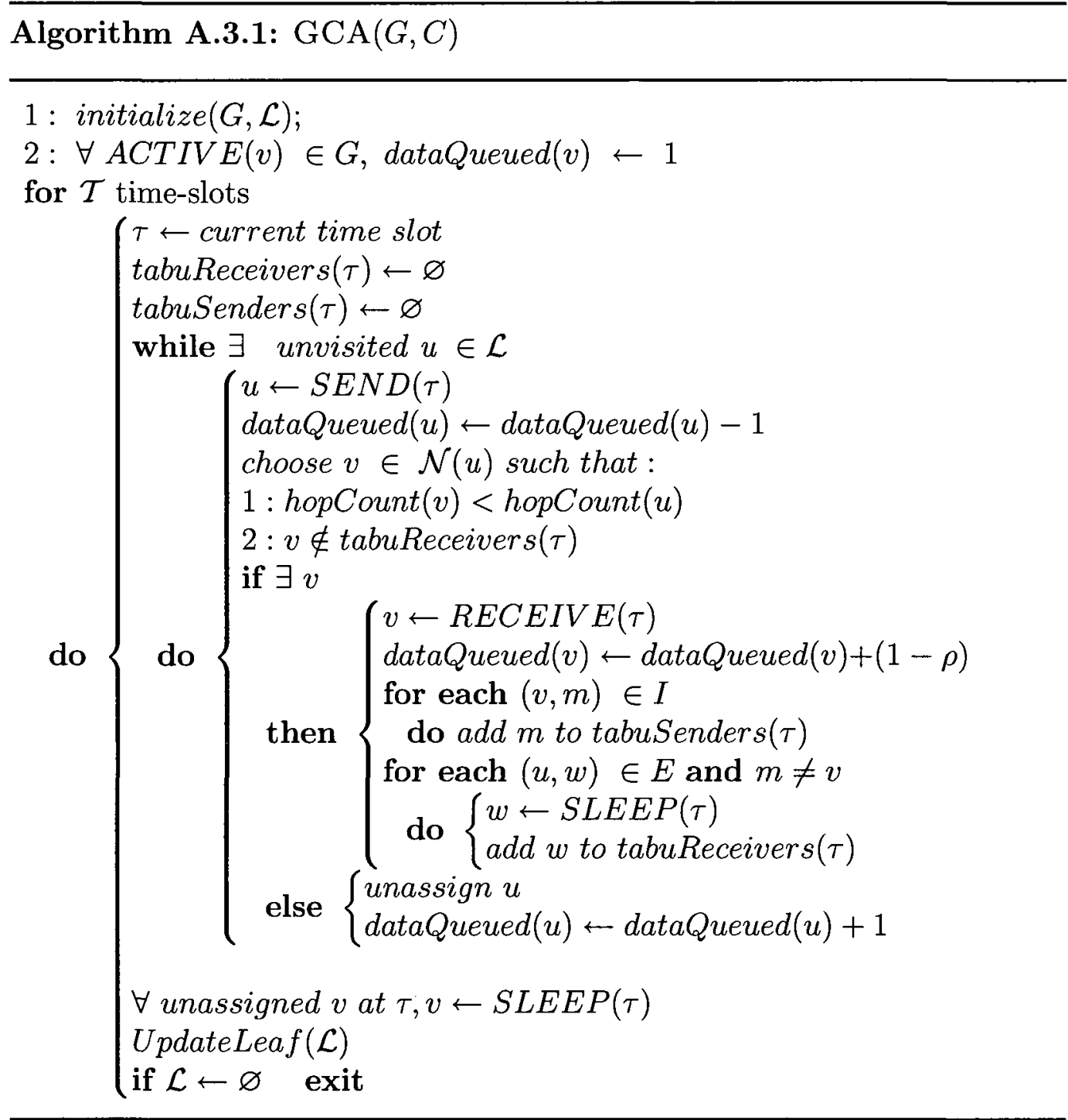




\section{A.4 Sensing Range Correlation-aware Scheduling}

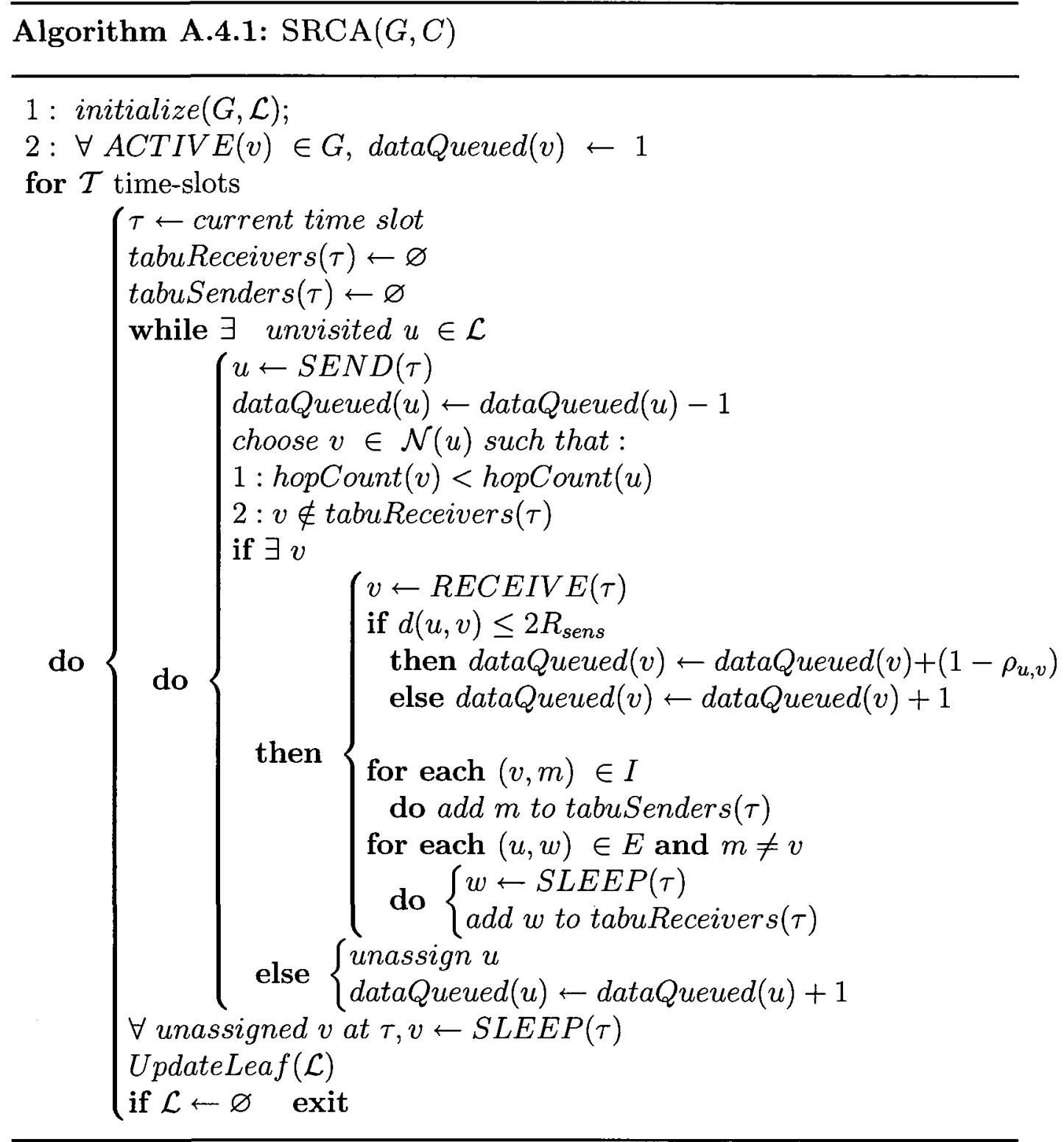




\section{A.5 Update Leafnodes}

\begin{tabular}{l}
\hline Algorithm A.5.1: $\operatorname{UpdateLeAF}(\mathcal{L})$ \\
for each $u \in \mathcal{L}$ \\
do $\left\{\begin{array}{c}\text { if dataQueued }(v)=0 \\
\text { then } \mathcal{L} \leftarrow \mathcal{L}-u\end{array}\right.$ \\
\hline
\end{tabular}

\section{A.6 Interference Handling}

Algorithm A.6.1: Interference handuing $(G, C)$

$$
\begin{aligned}
& \tau \leftarrow \text { current time slot } \\
& u \leftarrow S E N D(\tau) \\
& v \leftarrow R E C E I V E(\tau) \\
& \text { interference }(v)=0 \\
& \text { for all } m \in C \text { such that }(m, v) \in I \\
& \text { do }\left\{\begin{array}{l}
\text { if } m \text { is unallocated } \\
\text { then }\left\{\begin{array}{l}
\text { interference }(v) \leftarrow \text { interference }(v)+\text { weight }(m, v) \\
\text { if }(\text { wight }(u, v) / \text { interference }(v))<\beta \\
\text { then } \\
\text { add } m \text { to tabuSenders } \\
\text { interference }(v) \leftarrow \text { interference }(v)-\text { weight }(m, v)
\end{array}\right.
\end{array}\right.
\end{aligned}
$$




\section{Appendix B}

\section{Confidence-Interval Estimation}

Calculating the confidence interval is important in characterizing the measure of error in simulation results $[97,98]$. The sample mean, e.g. delay, of $R$ replications is given by:

$$
\bar{Y}=\frac{1}{R} \sum_{i=1}^{R} \bar{Y}_{i},
$$

where $\bar{Y}_{i}$ is the sample mean of replication $i$. In addition, the sample variance across $R$ replications is:

$$
S^{2}=\frac{1}{R-1} \sum_{i=1}^{R}\left(\bar{Y}_{i}-\bar{Y}\right)^{2} .
$$

The confidence interval is then:

$$
\bar{Y} \pm t_{\alpha / 2, R-1} \frac{S}{\sqrt{R}}
$$

such that

$$
\bar{Y}-t_{\alpha / 2, R-1} \frac{S}{\sqrt{R}} \leq \Theta \leq \bar{Y}+t_{\alpha / 2, R-1} \frac{S}{\sqrt{R}}
$$

where $t_{\alpha / 2, R-1}$ is the quantile of the $\mathbf{t}$ distribution with $R-1$ degree of freedom. 
The above method of calculating the confidence interval assumes a fixed R. In such case, for the same value of $\mathrm{R}$ the error specified by $t_{\alpha / 2, R-1} \frac{S}{\sqrt{R}}$ may change when simulation variables change. In order to have the error within a specific value $\pm \epsilon$ with probability $1-\alpha$, the number of replications $R$ must satisfy:

$$
R \geq\left(\frac{t_{\alpha / 2, R-1} S_{0}}{\epsilon}\right)^{2}
$$

where $S_{0}^{2}$ is the sample variance of $2 \leq R_{0} \leq R$ replications. Once the value of $R$ is specified then $R-R_{0}$ additional replications are performed to achieve the desired level of error.

An initial estimate of $\mathrm{R}$ can be found by:

$$
R \geq\left(\frac{z_{\alpha / 2} S_{0}}{\epsilon}\right)^{2}
$$




\section{Appendix $\mathrm{C}$}

\section{ns-2 Network Simulator}

\section{C.0.1 Introduction}

- A a discrete event simulator modeling network protocols.

- Supported platforms: Linux, Solaris, Windows, Mac.

- First Release: ns-2.0b17 in 1997.

- Current release: ns-2.34.

- Specify simulation, generate traces.

- Depends on Tcl/Tk, OTcl, TclCL.

- Functionalities:

- Wired:

* Routing: Distance Vector (DV), link state, static, multicast.

* Transport: TCP, UDP, RTP, SCTP. 
* Applications: HTTP, FTP, TELNET, RealAudio, Constant Bit Rate (CBR), Exponential.

- Wireless:

* Routing: Ad-hoc On-demand Distance Vector (AODV), DestinationSequenced Distance-Vector (DSDV), Dynamic Source Routing (DSR).

* MAC: 802.11, S-MAC, simple TDMA.

* Sensor Macs (External): DMAC, Z-MAC, Two Radio, LEACH.

* Radio Propagation: Free space, Two-way ground reflection, Shadowing.

* Energy: Transmit, Receive, Idle, Sleep, Switch. Implemented (MCU active and sleep).

- Queues: Drop-tail (FIFO), RED, CBQ, Fair Queueing (FQ), Stochastic Fair Queueing (SFQ), and Deficit Round-Robin (DRR).

- Pre-processing: Traffic and topology generation.

- Create simulation:

- Describe network, protocols, sources, sinks.

- Interface via OTcl which controls $\mathrm{C}++$.

- Execute simulation:

- Simulator maintains event list (packet list), executes next event (packet), repeats until done.

- Events happen instantly in virtual time but could take arbitrarily long real time. 
- Single thread of control, no locking, races, etc.

\section{C.0.2 Contributed code}

- PROGRESSIVE.

- OCSMACS.

- Scheduling schemes.

- Integrated routing-MAC.

- Realistic interference model which is based on additive SNR instead of the built-in capture model.

- Multichannel model, including interference across channels (Experimental).

- Topology and traffic generator. 


\section{Bibliography}

[1] I. Mahgoub and M. Ilyas, Smart Dust: Sensor Network Applications, Architecture and Design. CRC Press, 2006.

[2] H. Karl and A. Willig, Protocols and Architectures For Wireless Sensor Networks. Wiley, 2005.

[3] T. M. I. F. Akyildiz and K. R. Chowdhury, "Wireless Multimedia Sensor Networks: Applications and Testbeds," Proceedings of the IEEE, vol. 96, no. 10, pp. 1588-1605, 2008.

[4] J. Paek, K. Chintalapudi, R. Govindan, J. Caffrey, and S. Masri, "A wireless sensor network for structural health monitoring: performance and experience," in Proc. of the 2nd IEEE workshop on Embedded Networked Sensors, EmNets'05. Washington, DC, USA: IEEE Computer Society, 2005, pp. 1-9.

[5] D. J. Cook and S. K. Das, "Wireless Sensor Networks," Smart Environments: Technologies, Protocols, and Applications, vol. 1, pp. 751-756, Oct. 2004.

[6] F. Chiti, M. Ciabatti, G. Collodi, D. D. Palma, R. Fantacci, and A. Manes, "Design and application of enhanced communication protocols for wireless sensor networks operating in environmental monitoring," in IEEE International 
Conference on Communications, vol. 8, June 2006, pp. $3390-3395$.

[7] C.-Y. Chong and S. Kumar, "Sensor networks: evolution, opportunities, and challenges," in Proc. IEEE, vol. 91, Aug. 2003, pp. 1247 - 1256.

[8] J. Elson and D. Estrin, "Time synchronization for wireless sensor networks," in Proc. of the 15th International Parallel \& Distributed Processing Symposium, IPDPS'01, 2001, p. 186.

[9] ns-2 - The Network Simulator, http://www.isi.edu/nsnam/ns/.

[10] I. Rhee, A. Warrier, J. Min, and L. Xu, "DRAND: Distributed randomized TDMA scheduling for wireless ad-hoc networks," in Proc. 7th ACM international symposium on Mobile ad hoc networking and computing (MobiHoc), 2006, pp. 190-201.

[11] W. Ye, J. Heidemann, and D. Estrin, "Medium access control with coordinated adaptive sleeping for wireless sensor networks," IEEE/ACM Transactions on Networking, vol. 12, no. 3, pp. 493 - 506, June 2004.

[12] G. Lu, B. Krishnamachari, and C. S. Raghavendr, "An adaptive energy-efficient and low-latency MAC for data gathering in wireless sensor networks," in 18th International Parallel and Distributed Processing Symposium, 2004, pp. 224-.

[13] I. Rhee, A. Warrier, M. Aia, J. Min, and M. Sichitiu, "Z-MAC: A hybrid mac for wireless sensor networks," IEEE/ACM Transactions on Networking, vol. 16, no. 3, pp. $511-524$, June 2008. 
[14] I. Rhee, A. Warrier, M. Aia, and J. Min, "Z-MAC: a hybrid MAC for wireless sensor networks," in Proc. 3rd International Conference on Embedded Networked Sensor Systems, 2005, pp. 90 - 101.

[15] S. Phoha, T. LaPorta, and C. Griffin, Sensor Network Operations. IEEE Press and Wiley, 2006.

[16] G. Xing, X. Wang, Y. Zhang, C. Lu, R. Pless, and C. Gill, "Integrated coverage and connectivity configuration for energy conservation in sensor networks," ACM Transactions on Sensor Networks, pp. 36 - 72, 2005.

[17] A. Ruzzelli, G. O'Hare, R. Tynan, P. Cotan, and P. Havingat, "Protocol assessment issues in low duty cycle sensor networks: The switching energy," in IEEE International Conference on Sensor Networks, Ubiquitous, and Trustworthy Computing, 05-07 June 2006, pp. 136 - 143.

[18] M. Aboelaze and F. Aloul, "Current and future trends in sensor networks: a survey," in Second IFIP International Conference on Wireless and Optical Communications Networks (WOCN), 2005, pp. $551-555$.

[19] L. Kirousis, E. Kranakis, D. Krizanc, , and A. Pelc, "Power consumption in packet radio networks," Theor. Computer Sci., vol. 243, p. 289305, 2000.

[20] I. F. Akyildiz, W. Su, Y. Sankasubramaniam, and E. Cayirci, "Wireless sensor networks: A survey," IEEE Communications Magazine, vol. 40, no. 8, pp. 102 - 114, Aug. 2002. 
[21] M. Vieira, C. C. Jr., D. da Silva Jr., and J. da Mata, "Survey on wireless sensor network devices," in Proc. IEEE Emerging Technologies and Factory Automation Conference (ETFA), vol. 1, 2003, pp. 537 - 544 .

[22] M. Ilyas and I. Mahgoub, Handbook of Sensor Networks: Compact Wireless and Wired Sensing Systems. CRC Press, 2004.

[23] Crossbow Technology Inc. MICA2, http://www.xbow.com.

[24] E. Noeland and W. Tang, "Novel Sensor MAC Protocol Applied to Cayley and Manhattan Street Networks with CrossBow MICA2," in Proc. 3rd Annual IEEE Communications Society on Sensor and Ad Hoc Communications and Networks (SECON), 28-28 Sept. 2006, pp. $626-631$.

[25] S. Kulkarni, A. Iyer, and C. Rosenberg, "An address-light, integrated MAC and routing protocol for wireless sensor networks," IEEE/ACM Transactions on Networking, vol. 14, pp. 793 - 806, Aug. 2006.

[26] Z. Cheng, M. Perillo, and W. B. Heinzelman, "General network lifetime and cost models for evaluating sensor network deployment strategies," IEEE Transactions on Mobile Computing, vol. 7, no. 4, pp. 484 - 497, April 2008.

[27] S. Panichpapiboon, G. Ferrari, and O. Tonguz, "Optimal transmit power in wireless sensor networks," IEEE Transactions on Mobile Computing, vol. 5, pp. 1432 - 1447, Oct. 2006.

[28] E. H. Callaway, Wireless Sensor Networks: Architectures and Protocols. CRC Press, 2004. 
[29] Y. Sagduyu and A. Ephremides, "The problem of medium access control in wireless sensor networks," IEEE Wireless Communications, vol. 11, no. 6, pp. $44-53$, Dec. 2004.

[30] N. August and D. S. Ha, "Operation, system architectures, and physical layer design considerations of distributed MAC protocols for uwb," IEEE Transactions on Microwave Theory and Techniques, vol. 54, pp. 3001 - 3012, July 2006.

[31] Q. Liu, X. Wang, and G. Giannakis, "A cross-layer scheduling algorithm with QoS support in wireless networks," IEEE Transactions on Vehicular Technology, pp. $839-847$, May 2006.

[32] J. Misic, S. Shafi, and V. Misic, "Cross-layer activity management in an $802-$ 15.4 sensor network," IEEE Communications Magazine, vol. 44, pp. 131 - 136, Jan. 2006.

[33] W. Liu, Y. Zhang, W. Lou, and Y. Fang, "A robust and energy-efficient data dissemination framework for wireless sensor networks," Wireless Networks, vol. 12, no. 4, pp. 465-479, 2006.

[34] G. Zhou, T. He, J. Stankovic, and T. Abdelzaher, "RID: radio interference detection in wireless sensor networks," in Proc. of the 24th Annual Joint Conference of the IEEE Computer and Communications Societies, INFOCOM'05, 2005, p. 891.

[35] E. Duarte-Melo and M. Liu, "Analysis of energy consumption and lifetime of heterogeneous wireless sensor networks," in IEEE Global Telecommunications Conference (GLOBECOM), vol. 1, no. 1, Nov. 2002, pp. $21-25$. 
[36] I. Chatzigiannakis, A. Kinalis, and S. Nikoletseas, "Power conservation schemes for energy efficient data propagation in heterogeneous wireless sensor networks," in Proc. 38th Annual Simulation Symposium, 4-6 April 2005, pp. $60-71$.

[37] P. Galiotos, "Sleep/Active schedules as a tunable characteristic of a wireless sensor network," in International conference on Networking and Services (ICNS), 16-18 July 2006, p. 51.

[38] S. Hussain, T. Khan, and S. Zaidi, "Latency and energy efficient MAC (LEEMAC) protocol for event critical applications in wsns," in International Symposium on Collaborative Technologies and Systems (CTS), 14-17 May 2006, pp. $370-378$.

[39] S.-J. Kim, X. Wang, and M. Madihian, "Joint routing and medium access control for lifetime maximization of distributed wireless sensor networks," in IEEE International Conference on Communications, vol. 8, June 2006, pp. 3467 -3472 .

[40] J. Zhu, S. Chen, and B. Bensaou, "Adaptive medium access control for minimum energy reliable data delivery in wireless sensor networks," in Proc. of the 10th ACM Symposium on Modeling, analysis, and simulation of wireless and mobile systems. MSWiM '07. New York, NY, USA: ACM, 2007, pp. 352-359.

[41] H. Karvonen and C. Pomalaza-Raez, "A cross layer design of coding and awake/sleep periods in WSNS," in IEEE 17th International Symposium on Personal, Indoor and Mobile Radio Communications, Sept. 2006, pp. $1-5$. 
[42] K. Chowdhury, N. Nandiraju, D. Cavalcanti, and D. Agrawal, "CMAC: A multichannel energy efficient MAC for wireless sensor networks," in IEEE Wireless Communications and Networking Conference (WCNC), 3-6 April 2006, pp. 1172 $-1177$.

[43] A. V. den Bossche, T. Val, and E. Campo, "Proposition of a full deterministic medium access method for wireless network in a robotic application," in IEEE 63rd Vehicular Technology Conference (VTC 2006-Spring), 2006, pp. 279-283.

[44] I.-K. Rhee, J. Lee, J. Kim, E. Serpedin, and Y.-C. Wu, "Clock Synchronization in Wireless Sensor Networks: An Overview," Sensors, vol. 9, no. 1, pp. 56-85, 2009.

[45] "An energy efficient and accurate slot synchronization scheme for wireless sensor networks."

[46] B. Sundararaman, U. Buy, and A. Kshemkalyani, "Clock synchronization for wireless sensor networks: a survey," Ad Hoc Networks, vol. 3, no. 3, pp. 281-323, 2005.

[47] B. Han and G. Simon, "Optimizing Multi-hop Queries in ZigBee Based Multisink Sensor Networks," in Proc. of the 10th International Conference on Distributed Computing and Networking, ICDCN'09, 2009, pp. 294-305.

[48] F. Wang, P. Zeng, and H. Yu, "Slot Time Synchronization for TDMA-Based Ad Hoc Networks," vol. 2. IEEE Computer Society, 2008, pp. 544-548. 
[49] D. Yu, "A statistical study of neighbor node properties in ad hoc network," in Proc. of the 2002 International Conference on Parallel Processing Workshops, ICPPW'02. IEEE Computer Society, 2002, p. 103.

[50] N. Riga, I. Matta, and A. Bestavros, "A geometric approach to slot alignment in wireless sensor networks," in Proc. of the IEEE Global Telecommunications Conference. GLOBECOM'07, 2007, pp. 817-822.

[51] W. Heinzelman, A. Chandrakasan, and H. Balakrishnan, "An ApplicationSpecific Protocol Architecture for Wireless Microsensor Networks," IEEE Transactions on Wireless Communications, vol. 1, no. 4, pp. 660 -670, Oct. 2002.

[52] T. S. Rappaport, Wireless Communications: Principles and Practice. Prentice Hall, 2001.

[53] T. van Dam. and K. Langendoen, "An adaptive energy-efficient MAC protocol for wireless sensor networks," in Proc. 1st International Conference on Embedded Networked Sensor Systems, 2003, pp. $171-180$.

[54] M. Miller and N. Vaidya, "Power save mechanisms for multi-hop wireless networks," in Proc. First International Conference on Broadband Networks (BroadNets), 2004, pp. $518-526$.

[55] M. Brownfield, K. Mehrjoo, A. Fayez, and N. Davis, "Wireless sensor network energy-adaptive MAC protocol," in 3rd IEEE Consumer Communications and Networking Conference (CCNC), 8-10 Jan. 2006, pp. $778-782$. 
[56] B. Chen, K. Jamieson, H. Balakrishnan, and R. Morris, "Span: an energyefficient coordination algorithm for topology maintenance in ad hoc wireless networks," Wireless Networks, vol. 8, no. 5, pp. 481-494, 2002.

[57] S. Coleri, A. Puri, and P. Varaiya, "PEDAMACS: Power efficient and delay aware medium access protocol for sensor networks," IEEE Transactions on Mobile Computing, vol. 5, no. 7, pp. 920-930, 2006.

[58] C. Rohl, H. Woesner, and A. Wolisz, "A Short Look on Power Saving Mechanisms in the Wireless LAN Standard IEEE 802.11," Advances in Wireless Communications, pp. 219-226, 1998.

[59] R. Subramanian and F. Fekri, "Sleep scheduling and lifetime maximization in sensor networks: fundamental limits and optimal solutions," in Proc. of the 5th international conference on Information processing in sensor networks, IPSN'06. New York, NY, USA: ACM, 2006, pp. 218-225.

[60] R. Biswas, V. Jain, C. Ghosh, and D. Agrawal, "On-demand reliable medium access in sensor networks," in International Symposium on a World of Wireless, Mobile and Multimedia Networks (WoWMoM), 26-29 June 2006.

[61] C. Schurgers, V. Tsiatsis, and M. Srivastava, "STEM: Topology management for energy efficient sensor networks," in Proc. IEEE Aerospace Conference, vol. 3, 2002, pp. 3-1099-3-1108.

[62] M. Miller and N. Vaidya, "Minimizing energy consumption in sensor networks using a wakeup radio," in IEEE Wireless Communications and Networking Conference, vol. 4, 21-25 March 2004, pp. $2335-2340$. 
[63] C. Schurgers, V. Tsiatsis, S. Ganeriwal, and M. Srivastava, "Optimizing sensor networks in the energy-latency-density design space," IEEE Transactions on Mobile Computing, vol. 1, no. 1, pp. 70 - 80, Jan.-March 2002.

[64] _ _ , "Topology management for sensor networks: Exploiting latency and density," in Proc. the 3rd ACM international symposium on Mobile ad hoc networking $\mathscr{G}$ computing, 2002, pp. $135-145$.

[65] M. Miller and N. Vaidya, "A MAC protocol to reduce sensor network energy consumption using a wakeup radio," IEEE Transactions on Mobile Computing, vol. 4, no. 3, pp. 228 - 242, May-June 2005.

[66] A. Bachir, D. Barthel, M. Heusse, and A. Duda, "Micro-Frame Preamble MAC for multihop wireless sensor networks," in IEEE International Conference on Communications, vol. 7, June 2006, pp. $3365-3370$.

[67] A. El-Hoiydi, "Aloha with Preamble Sampling for Sporadic Traffic in Ad Hoc Wireless Sensor Networks," in Proc. IEEE International Conference on Communications, vol. 5, 2002, pp. $3418-3423$.

[68] J. Polastre, J. Hill, and D. Culler, "Versatile low power media access for wireless sensor networks," in Proc. 2nd International Conference on Embedded Networked Sensor Systems, 2004, pp. 95 - 107.

[69] X. Shi, G. Stromberg, Y. Gsottberger, T. Sturm, I. AG, and G. Munich, "Wakeup-frame scheme for ultra low power wireless transceivers," in IEEE Global Telecommunications Conference (GLOBECOM), vol. 6, 2004. 
[70] X. Shi and G. Stromberg, "SyncWUF: An Ultra Low-Power MAC Protocol for Wireless Sensor Networks," IEEE Transactions on Mobile Computing, pp. $115-125,2007$.

[71] A. El-Hoiydi and J.-D. Decotignie, "WiseMAC: an ultra low power MAC protocol for the downlink of infrastructure wireless sensor networks," in Proc. 9th International Symposium on Computers and Communications (ISCC), vol. 1, 28 June-1 July 2004, pp. $244-251$.

[72] C. Enz, A. El-Hoiydi, J.-D. Decotignie, and V. Peiris, "WiseNET: an ultralowpower wireless sensor network solution," Computer, vol. 37, no. 8, pp. $62-70$, Aug. 2004.

[73] A. El-Hoiydi, C. Arm, R. Caseiro, S. Cserveny, J.-D. Decotignie, C. Enz, F. Giroud, S. Gyger, E. Leroux, T. Melly, V. Peiris, F. Pengg, P.-D. Pfister, N. Raemy, A. Ribordy, D. Ruffieux, and P. Volet, "The ultra low-power WiseNET system," in Proc. Conference on Design, Automation and Test in Europe (DATE), 2006, pp. $971-976$.

[74] S. Ramanathan, "A unified framework and algorithm for (T/F/C)DMA channel assignment in wireless networks," in Proc. 6th Annual Joint Conference of the IEEE Computer and Communications Societies (INFOCOM), 1997, pp. 900907.

[75] K. Jamieson, H. Balakrishnan, and Y. C. Tay, "Sift: A mac protocol for eventdriven wireless sensor networks," in Proc. European Conference on Wireless Sensor Networks (EWSN), 2006, pp. 260-275. 
[76] Ephremides and O. A. Mowafi, "Analysis of a hybrid access scheme for buffered users-probabilistic time division," IEEE Transactions on Software Engineering, vol. SE-8, no. 1, pp. $52-61,1982$.

[77] J.-L. Wu and S.-T. Wang, "A two-tier data dissemination model for large-scale wireless sensor networks," in Proc. 8th Annual International Conference on Mobile Computing and Networking, 2002, pp. $148-159$.

[78] J. Zhao and A. Erdogan, "A novel self-organizing hybrid network protocol for wireless sensor networks," in First NASA/ESA Conference on Adaptive Hardware and Systems (AHS), 15-18 June 2006, pp. $412-419$.

[79] S. Ramanathan and E. Lloyd, "Scheduling algorithms for multihop radio networks," IEEE/ACM Transactions on Networking, vol. 1, no. 2, pp. 166 - 177, April 1993.

[80] S. C. Ergen and P. Varaiya, "TDMA scheduling algorithms for sensor networks," Department of Electrical Engineering and Computer Sciences University of California, Berkeley, Technical Report, 2005.

[81] T. Moscibroda and R. Wattenhofer, "Coloring unstructured radio networks," in Proc. 17th Annual ACM Symposium on Parallelism in Algorithms and Architectures, 2005, pp. $39-48$.

[82] H. Li, P. Shenoy, and K. Ramamritham, "Scheduling communication in realtime sensor applications," in Proc. 10th IEEE Real-Time and Embedded Technology and Applications Symposium (RTAS), 25-28 May 2004, pp. 10 - 18. 
[83] S. Gandham, M. Dawande, and R. Prakash, "Link scheduling in sensor networks: distributed edge coloring revisited," in Proc. IEEE 24th Annual Joint Conference of the IEEE Computer and Communications Societies (INFOCOM), vol. 4, March 2005, pp. 2492- 2501.

[84] S. Cui, R. Madan, A. J. Goldsmith, and S. Lall, "Energy-delay tradeoffs for data collection in TDMA-based sensor networks," in The 40th annual IEEE International Conference on Communications, vol. 5, May 2005, pp. 3278 3284 .

[85] R. Madan, S. Cui, S. Lall, and N. Goldsmith, "Cross-layer design for lifetime maximization in interference-limited wireless sensor networks," IEEE Transactions on Wireless Communications, vol. 5, no. 11, pp. 3142 - 3152, November 2006.

[86] G. Jolly and M. Younis, "An energy-efficient scalable and collision-free MAC layer protocol for wireless sensor networks," Wireless Communications and Mobile Computing, vol. 5, no. 3, pp. 285-304, 2005.

[87] J. Mao, Z. Wu, and X. Wu, "A TDMA scheduling scheme for many-to-one communications in wireless sensor networks," Computer Communications, vol. 30, no. 4, pp. 863-872, February 2007.

[88] A. Karnik, A. Iyer, and C. Rosenberg, "What is the right model for wireless channel interference?" IEEE Transactions on Wireless Communications, vol. 8, pp. $2662-2671$, May 2009. 
[89] W. Su and I. Akyildiz, "Time-diffusion synchronization protocol for wireless sensor networks," IEEE/ACM Transactions on Networking, vol. 13, pp. 384 397, April 2005.

[90] M. Maróti, B. Kusy, G. Simon, and A. Lédeczi, "The flooding time synchronization protocol," in SenSys '04: Proceedings of the 2nd international conference on Embedded networked sensor systems, 2004, pp. 39-49.

[91] J. Elson, L. Girod, and D. Estrin, "Fine-grained network time synchronization using reference broadcasts," in OSDI '02: Proceedings of the 5th symposium on Operating systems design and implementation. New York, NY, USA: ACM, 2002, pp. 147-163.

[92] A. Barnawi and R. Hafez, "An Adaptive MAC Protocol for Infrastructure Wireless Sensor Networks," in Proc. International Conference on Sensor Technologies and Applications (SENSORCOMM), Oct. 2007, pp. $301-306$.

[93] —_, "A Time \& Energy Efficient Topology Discovery and Scheduling Protocol for Wireless Sensor Networks," in Proc. of the 2009 IEEE/IFIP International Symposium on Embedded and Pervasive Systems, Aug. 2009.

[94] —_, "TDMA Scheduling for Data Gathering in Wireless Sensor Networks," in Proc. International Conference on Wireless Networks (ICWN'08). Part of the 2008 World Congress in Computer Science, Computer Engineering, and Applied Computing (Worldcomp'08), vol. 1, Las Vegas, Nevada, USA, July 14-17 2008, pp. $94-100$. 
[95] S. Bandyopadhyay, Q. Tian, and E. Coyle, "Spatio-temporal sampling rates and energy efficiency in wireless sensor networks," IEEE/ACM Transactions on Networking (TON), vol. 13, no. 6, pp. 1339-1352, 2005.

[96] Y. Wang and J. J. Garcia-Luna-Aceve, "Modeling of collision avoidance protocols in single-channel multihop wireless networks," Wireless Networks, vol. 10, pp. $495-506,2004$.

[97] J. Banks, J. S. Carson, B. L. Nelson, and D. M. Nicol, Discrete-Event System Simulation. Prentice Hall, 2009.

[98] J. Banks, Handbook of Simulation: Principles, Methodology, Advances, Applications, and Practice. Wiley-Interscience, 1998.

[99] Texas Instruments. CC1000 - Single Chip Very Low Power RF Transceiver, http://www.ti.com.

[100] Atmel - Atmega128, http://www.atmel.com.

[101] L. Krishnamachari, D. Estrin, and S. Wicker, "The impact of data aggregation in wireless sensor networks," in International Workshop of Distributed Event Based Systems (DEBS), July 2002, pp. 575-578.

[102] C. Intanagonwiwat, D. Estrin, R. Govindan, and J. Heidemann, "Impact of network density on data aggregation in wireless sensor networks," in International Conference on Distributed Computing Systems (ICDCS), 2002, pp. 457-458.

[103] V. Erramilli, I. Malta, and A. Bestavros, "On the interaction between data aggregation and topology control in wireless sensor networks," in First Annual 
IEEE Communications Society Conference on Sensor and Ad Hoc Communications and Networks, IEEE SECON, Oct. 2004, pp. 557-565.

[104] Y. Chen, A. Liestman, and J. Liu, "A hierarchical energy-efficient framework for data aggregation in wireless sensor networks," IEEE Transactions on Vehicular Technology, vol. 55, no. 3, pp. 789-796, May 2006.

[105] C. Hua and T.-S. P. Yum, "Data aggregated maximum lifetime routing for wireless sensor networkss," Ad Hoc Networks, vol. 6, no. 3, pp. 380-392, May 2008.

[106] E. F. Nakamura, A. A. F. Loureiro, and A. C. Frery, "Information fusion for wireless sensor networks: Methods, models, and classifications," ACM Computing Surveys, vol. 39, no. 3, p. 9, 2007.

[107] Y. Zhu, R. Vedantham, S. Park, and R. Sivakumar, "A scalable correlation aware aggregation strategy for wireless sensor networks," Information Fusion, vol. 9 , no. 3 , pp. 354-369, 2008.

[108] X. Tang and J. Xu, "Optimizing lifetime for continuous data aggregation with precision guarantees in wireless sensor networks," IEEE/ACM Trans. Netw., vol. 16, no. 4, pp. 904-917, 2008.

[109] A. Polychronopoulos, M. Tsogas, A. Amditis, and L. Andreone, "Sensor Fusion for Predicting Vehicles' Path for Collision Avoidance Systems," IEEE Transactions on Intelligent Transportation Systems, vol. 8, no. 3, pp. 549-562, 2007. 
[110] P. von Rickenbach and R. Wattenhofer, "Gathering correlated data in sensor networks," in Proc. of the 2004 joint workshop on Foundations of mobile computing. DIALM-POMC'04. New York, NY, USA: ACM, 2004, pp. 60-66.

[111] S. Pattem, B. Krishnamachari, and R. Govindan, "The impact of spatial correlation on routing with compression in wireless sensor networks," ACM Transactions Sensor Networks, vol. 4, no. 4, pp. 1-33, 2008.

[112] A. Barnawi and R. Hafez, "Performance of TDMA scheduling algorithms in the presence of data correlation in sensor networks," in Proc. IEEE Canadian Conference on Electrical and Computer Engineering (CCECE), Niagara Falls, ON, 4-7 May 2008, pp. 675-680.

[113] R. Subramanian, H. Pishro-Nik, and F. Fekri, "Clustering-based correlation aware data aggregation for distributed sensor networks," in IEEE Global Telecommunications Conference (GLOBECOM), vol. 6, 2005.

[114] C. Hua and T.-S. Yum, "Optimal Routing and Data Aggregation for Maximizing Lifetime of Wireless Sensor Networks," IEEE/ACM Transactions on Networking, vol. 16, no. 4, pp. 892-903, Aug. 2008.

[115] H. Luo, Y. Liu, and S. Das, "Routing Correlated Data with Fusion Cost in Wireless Sensor Networks," IEEE Transactions on Mobile Computing, vol. 5, no. 11, pp. 1620-1632, Nov. 2006.

[116] S. Lindsey, C. Raghavendra, and K. Sivalingam, "Data gathering algorithms in sensor networks using energy metrics," IEEE Transactions on Parallel and Distributed Systems, vol. 13, no. 9, Sep 2002. 
[117] S. Lindsey and C. Raghavendra, "PEGASIS: Power-efficient gathering in sensor information systems," in Proc. IEEE Aerospace Conference, vol. 3, 2002, pp. $3-1125-3-1130$.

[118] T. M. Cover and J. A. Thomas, Elements of Information Theory. WileyInterscience, 1991.

[119] Crossbow Technology Inc. IMB400 - Imote2 Multimedia Board, http://www.xbow.com.

[120] Crossbow Technology Inc. Imote2, http://www.xbow.com.

[121] S. Bhatti, J. Carlson, H. Dai, J. Deng, J. Rose, A. Sheth, B. Shucker, C. Gruenwald, A. Torgerson, and R. Han, "Mantis os: an embedded multithreaded operating system for wireless micro sensor platforms," Mobile Networks and Applications, vol. 10, no. 4, pp. 563-579, 2005.

[122] C. M. Sadler and M. Martonosi, "Data compression algorithms for energyconstrained devices in delay tolerant networks," in Proc. of the 4th international conference on Embedded networked sensor systems. SenSys'06, 2006, pp. 265278.

[123] Texas Instruments. MSP430x161x - Mixed Signal Microcontroller Datasheet, http://www.ti.com. 